\title{
Charge transfer and storage in nanostructures
}

\author{
P. R. Bandaru*, H. Yamada, R. Narayanan, and M. Hoefer \\ Program in Materials Science, Department of Mechanical Engineering, \\ University of California, San Diego, La Jolla, CA 92093-0411
}

*Corresponding Author: Prabhakar R. Bandaru

Address: Room 258, Engineering 2

Department of Mechanical Engineering,

9500 Gilman Drive, MC 0411

UC, San Diego,

La Jolla, CA 92093-0411

Phone: (858) 534-5325

Fax: (858) 534-5698

E-mail: pbandaru@ucsd.edu 


\section{Abstract}

Efficient storage and conversion of electrical charge in materials, to a voltage and current, provides the basis for batteries and capacitors. Given the widespread usage of portable electronics there is a continual need to further enhance the energy and power density of such devices, which could be accomplished through the use of nanostructured materials. The large surface area to volume ratio and the possibilities of new materials physics and chemistry provide the rationale for their use and is discussed. The former aspect considers the relevance to the area-dependent capacitance as well as the parasitic elements that reduce the charge and energy delivery from the theoretical maximum values. Specific instances of electrode materials, as well as the electrode-electrolyte interface and electrolyte properties, with respect to their capability and prospects are examined. Alternate internal and external surface dependent Faradaic reactions and concomitant pseudocapacitance based mechanisms, seem to have the ability to bridge the large energy densities of batteries to the power density of the capacitors perhaps helping in realizing a truly useful hybrid device. While much of the report relates to presently used devices such as Li-ion batteries and activated carbon based electrochemical capacitors, the relevant principles are shown to be valid for other types of charge conversion agents such as photoelectrochemical and dye-sensitized solar cells. The review also considers perspectives on alternate materials and architectures.

Keywords: Charge storage, electrochemical capacitors, batteries, pseudocapacitance, quantum capacitance, Thin layer electrochemistry 


\section{$\underline{\text { Table of Contents }}$}

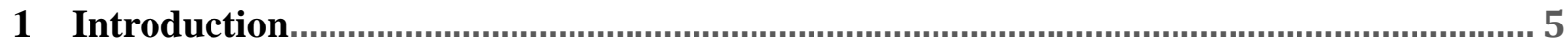

1.1 Characteristics of the nanoscale: Classical influences …...................................................... 7

1.2 Characteristics of the nanoscale: Quantum effects .............................................................. 9

2 Charge storage devices: Batteries and Capacitors .................................................... 15

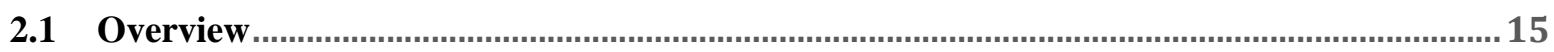

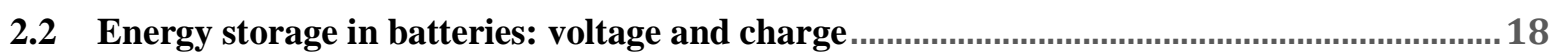

2.2.1 Charge transfer in solids, with emphasis on reversible intercalation ...................................22

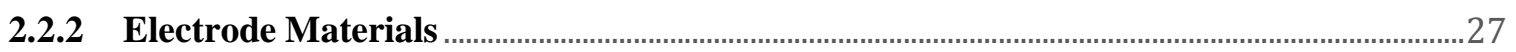

2.2.3 The influence of the electrolyte/ion-transfer medium ................................................................... 31

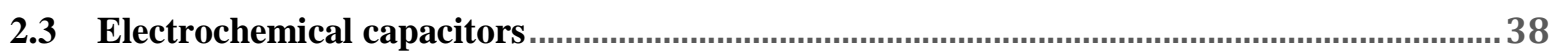

2.3.1 The double layer ….................................................................................................................................... 41

2.3.2 Additional capacitances modulate double layer capacitance ...................................................4 45

2.3.3 New electrolytes for electrochemical capacitors: Ionic Liquids ……......................................... 48

2.4 Charge capacity of electrochemical devices.................................................................50

2.4.1 State of the art and progress in enhancing charge \& energy storage .....................................51

2.4.2 Reaching the limits of charge storage ………........................................................................................5

2.4.3 Performance Metrics: The proper reporting of energy density …….......................................60

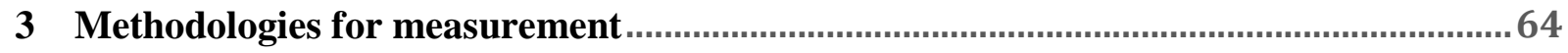

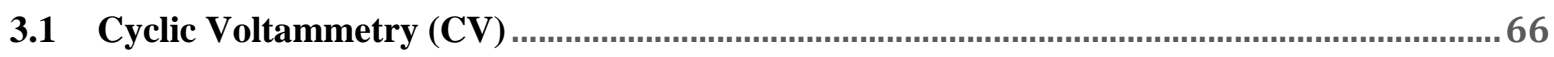

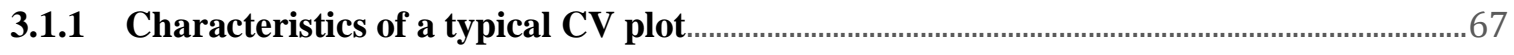

3.1.2 Reversibility, irreversibility, and quasi- reversibility ……......................................................... 71

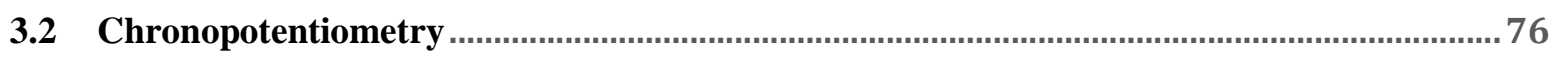

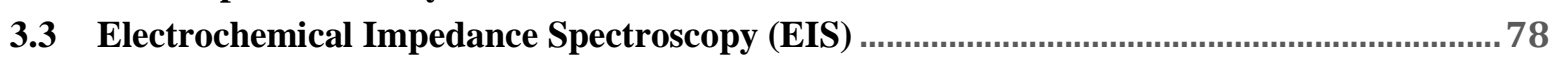

3.3.1 An EIS application: Self-discharge in electrochemical capacitors.........................................82

3.3.2 Components of the impedance

3.3.3 Capacitive dispersion ……........................................................................................................................ 85

4 Influence of the electrodes in charge transfer.................................................. 89

4.1 Charge carrier concentration in the electrode .................................................................. 90

4.2 The thermodynamics of charge transfer in a redox couple ............................................. 92

4.3 The electrical current between the electrode and the electrolyte ......................................97

4.4 Surface processes $v s$. Drift-Diffusion based processes ................................................... 99

4.5 The practical surface state of an electrode, e.g., the SEI (Solid Electrolyte Interface).......106

4.6 Charge transfer in semiconductors ........................................................................111

4.6.1 Charge configuration at a semiconductor interface …................................................

4.6.2 Charge transfer through the Semiconductor-Electrolyte interface ................................... 118

4.7 Charge transfer induced by photons: photoelectrochemical cells....................................122

4.8 Specific characteristics of nanostructured electrodes ...............................................125 


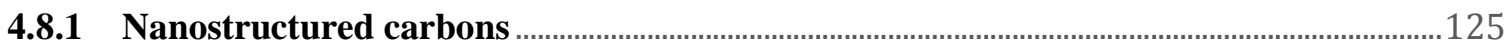

4.8.2 Non-ideality: Charges at defects ................................................................................................129

4.8.3 Influence of defects on bandstructure and charge capacity ……............................................135

4.8.4 Wetting and roughness of the electrodes …...............................................................................137

4.9 Porosity in Electrodes: A metric for high capacitance density .........................................139

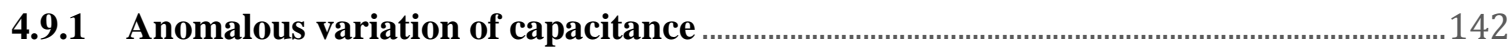

4.10 Chemical capacitance in nanostructures: Dye sensitized solar cells ..............................145

4.11 Quantum capacitance ............................................................................................153

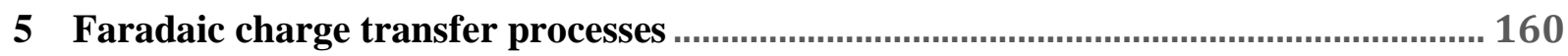

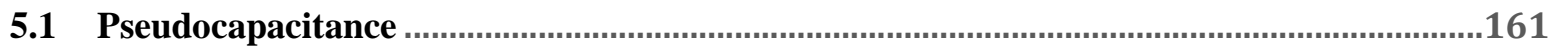

5.1.1 Criteria for maximal pseudocapacitance ……........................................................................... 165

5.1.2 Underlying mechanisms of adsorption pseudocapacitance ……...............................................168

5.1.3 The relation of pseudocapacitance to battery-like behavior ..................................................175

5.1.4 Intercalation pseudocapacitance (Inner \& Outer Electrode Areas?) ................................... 176

5.2 Faradaic energy storage in nanoscale confined electrolytes ..........................................182

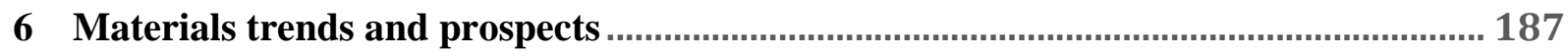

6.1 Electroactive polymers for high charge density and capacitance .....................................188

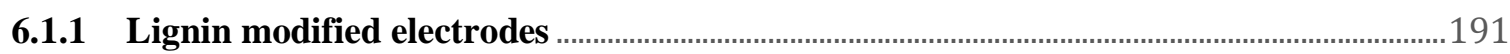

6.1.2 Polymer - Inorganic oxide hybrids ...................................................................................................194

6.2 Developing novel architectures for charge and energy storage .........................................195

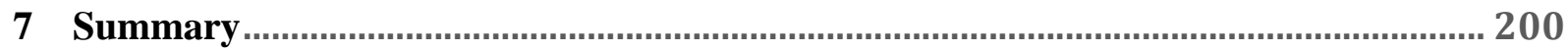

8 References 


\section{Introduction}

Human prosperity has been directly linked to the ability to efficiently use energy, and requires both energy generation and storage. Concerns related to the environmental impact as well as the possible resource crunch underlying fossil-based fuels has been a major motivator for the development of alternative, renewable, energy sources. To enhance the viability of such sources, as well as to increase the utility of conventional energy sources, it is necessary to provide compatible materials and devices that can store the energy for later use. In this context, recent advances in nanoscience and technology have been explored to gauge whether their use could facilitate improvements in the energy storage capacity. Given that most energy is utilized in the form of electricity and that the source of energy often resides at the level of chemical interactions involving electrons and ions, the notion of charge transfer and charge based energy storage is prominent.

Broadly, energy storage in the electrochemical sense has been conventionally classified into (a) batteries, or (b) capacitors, in terms of whether electrical charge is harnessed mostly in a thermodynamic sense (in terms of a net free energy change, of the material undergoing an electrochemical reaction) or in a kinetic sense (in terms of transducing the charge at an interface into an electrical current). From a practical perspective, batteries incorporates devices with high energy density ( $100 \mathrm{Wh} / \mathrm{kg}$ ) and relatively low power (rate of energy uptake/release) density ( $1 \mathrm{~kW} / \mathrm{kg}$ ), while capacitors comprise media with contrary attributes, i.e., relatively lower energy density $(\sim 10 \mathrm{Wh} / \mathrm{kg})$ and higher power density $(\sim 10 \mathrm{~kW} / \mathrm{kg})[1,2]$. (For an excellent perspective on the meaning of such numbers, consider the book: Sustainable Energy[3], e.g., the hydrocarbon based fuel for cars has a calorific value of $\sim 8 \mathrm{kWh} / \mathrm{kg}$ or that the typical power 
consumed by a $100 \mathrm{~W}$ light bulb (say, $50 \mathrm{~g}$ in weight) would be of the order $2 \mathrm{~kW} / \mathrm{kg}$ ) The overarching technological imperative in energy storage is then to devise intermediate devices, combining the best of both batteries and capacitors. Indeed, there has been frequent utilization of mutual principles, e.g., the invoking of an intercalation pseudocapacitance (see Section 5.1.4) for the fabrication of electrochemical capacitors.

We will then consider the major technological imperatives for the scientific study of charge storage, viz., through exploring the principles of batteries and capacitors. We broadly survey and understand the scientific rationale behind the modalities of charge creation and manipulation for electrochemical energy storage in nanostructures. We will attempt to understand the aspects that are unique to the nanoscale for it is to be expected that the relevant length scales bridge atomistic aspects to macro-scale utilization. Yet another objective is to understand (a) where the charge is stored - on the surface or in the volume/bulk, (b) the specific reaction sites for charge transfer in an electrochemical device, and their relevance for energy storage. Insights into the mechanism of charge transfer may be obtained through a consideration of the combined as well as separate influences of (a) adsorption, (b) accumulation, and (c) electrochemical reaction processes. The nature and origin of the reaction sites in materials will then be probed, with respect to whether or not they are defect induced. It seems possible, e.g., from an examination of the literature, that defects were involved in the charge storage (through the reported capacitance) in the use of materials such activated carbon, conducting polymers, metal oxides $\left(\mathrm{RuO}_{2}\right)$, nanotubes, etc., However, the result have not been reported in the defect context and we hope that the discussed principles would elucidate such influences. The possibilities of defect and charge engineering through various techniques, e.g., plasma 
processing[4,5], will then be discussed. In many such aspects, bridging atomistic scale characteristics to the macroscale and specific manifestation in device performance needs to be considered. This demands, for charge storage, an understanding of both classical electro-statics/dynamics as well as fundamental quantum mechanics issues. While Li ion based charge storage and underlying mechanisms form the basis of discussion for batteries, carbon-based materials will be considered for capacitive storage, for reasons related to their prevalent wide usage.

\section{nanoscale: Classical influences}

At the very outset, the term "nanoscale" has been conventionally used to refer to size scales in the range of $1 \mathrm{~nm}-100 \mathrm{~nm}$. On such a codified basis[6] lower dimensional nanoparticles (defined through nanoscale features in all three dimensions), nanofibers/nanotubes (with nanoscale features in two dimensions), and nanoplates (nanoscale in one-dimension, e.g., graphene) have all been shown to be pertinent for energy storage. Consequently, the nanoscale implies a large surface area to volume ratio (being inversely proportional to the length scale) indicating that surfaces are more relevant and an enhanced role for capacitive storage (since the electrostatic capacitance is directly proportional to the area) - see Section 2.3. Indeed, it is sometimes proclaimed that nanomaterials are "all surface". However, from the point of view of modifying fundamental characteristics, such as the voltage that could be obtained from a given nanoscale system, the surface may only have a small influence. For instance, the electrical potential change $(\Delta V)$ pertinent to an electrochemical reaction is often considered in terms of a Gibbs free energy change $(\Delta G)$ and is given by $(=-\Delta G / n F)$, with $n$ being the number of electrons involved/transferred and $F$ the Faraday constant $(96,487 \mathrm{C} /$ mole). However, a surface 
energy term proportional to the surface tension $(\gamma)$ /radius of the particle $(r)$ - may need to be added as well. However, for $1-100 \mathrm{~nm}$ radius nanoparticles with typical $\gamma \square \square$ of $0.1 \mathrm{~J} / \mathrm{m}^{2}$, an additional potential in the range of $0.001-0.1 \mathrm{~V}$ was estimated[7], implying negligible surface related effects. However, electrical charge may be developed at the interfaces of particles with different sizes due to such surface energy differences. The confinement of charge at such small length scales (see the next section) could influence the energy distributions of the surrounding material and may also play a role in modifying the matrix/electrolyte conductivity as well as ion/electrochemical storage (see Section 2.2.2).

A related large exposed surface would also imply a larger electrode solid - electrolyte interface (SEI) layer - due to the related electrochemical potential mismatch between the electrode and electrolyte, which consists of electrolyte/electrode reaction products and has been implicated in reducing the electrochemical kinetics (see Section 4.3). However, the formation of such layers may alleviate mechanical strains, e.g., pertinent to intercalation for lithium ion batteries (Section 2.2.1), and contribute to enhanced reliability on repeated cycling[8]. An interesting example, in this regard, was the observation that a small size does seem to make a difference, as in the intercalation of $\mathrm{Li}^{+}$ions (of radius $0.07 \mathrm{~nm}$ ) (see Section 2.2.1). It was observed that the amount of $\mathrm{Li}$ that could be inserted into $20 \mathrm{~nm}$ size $\alpha-\mathrm{Fe}_{2} \mathrm{O}_{3}$ particles, prior to structural change, was 20 times larger than that seen through the use of $500 \mathrm{~nm}$ particles[9]. While larger particles are more susceptible to phase changes, there is a prevalent notion that smaller particles more easily accommodate the strains through overall volume changes. While such size-dependent efficiency was also proposed for $\mathrm{Na}$ intercalation in graphite platelets, more 
specific studies would need to confirm the critical particle diameter and the energy involved for such changes, in addition to considering the particle packing and surroundings.

The rate of bulk charge storage, (considered in terms of power density) as would be relevant to batteries, may be improved through the use of nanostructures from the conventional assumption that the diffusion lengths of the electrons and ions would be reduced. However, it is far from clear whether such diffusional characteristics improve with lower dimensionality, e.g., it has been indicated that nanoparticles may have inferior energy discharge capabilities compared to nanowires of the same diameter[10]. Additionally, in the case of batteries, the inferior packing density of nanoscale objects may necessitate a larger number of such objects to achieve an equivalent energy density, considered per unit mass. Therefore, the volumetric energy density (reckoned in terms of energy/unit volume, i.e., $\mathrm{Wh} / \mathrm{m}^{3}$ or $\mathrm{Wh} / \mathrm{l}$ ) may be a more reliable metric (also see Section 2.4.3). The problem of interconnection of the nanoscale moieties to the current collector, which interfaces to an external three-dimensional architecture would also be a major consideration and has been implicated in polarization effects[7], through the manifestation of a large voltage hysteresis between the charge and discharge cycles. However, when nanoparticles are tightly packed there is a possibility of the space charge layers being coupled, and promoting ionic conductivity within the layers. Indeed, related effects such as heterogeneous doping have been invoked to enhance electrode conductivity: Section 2.2.2). The consideration of electronic energy levels in nanomaterials then brings forth issues related to energy confinement and quantum mechanical characteristics. 


\section{nanoscale: Quantum effects}

It is then not very evident [8] that nanoscale characteristics are clearly desirable for electrochemical storage, from the point of view of surface characteristics. However, when carrier confinement effects have to be considered - with the implication of new physical realms (falling broadly under the purview of quantum mechanical effects), there would be an opportunity to harness new realms of science. Essentially, even nanoscale objects, such as most Si nanowires, can be modeled as bulk/3-D materials, as long as quantum effects are irrelevant, i.e., any part of the material behaves as any other, while for lower dimensional material each part could be considered distinct. The implication is that, e.g., while the carrier density $(n)$ would always be in terms of number/unit volume, the way the volume is specified would be characteristic of the dimensionality. Consequently, while a bulk Si or a Si quantum wire may have the same carrier density the arrangement of the carriers (reckoned in terms of a density of states: DOS, or number of carriers per unit energy) would be different in the two manifestations, e.g., more (/less) electrons are accommodated at lower (/higher) energies in a nanowire exhibiting quantum effects, corresponding to the transition to discrete (atomic) energy levels and the absence of an extended band of energy levels. The mathematical aspects of the arrangement of the electrons in nanostructures vis-à-vis bulk materials, with respect to the DOS, have been well discussed, e.g., in Reference 11 [11].

An example of the practical necessity for the consideration of quantum effects is the manifestation of an additional quantum capacitance $\left(C_{Q}\right)$ - in series with the electrostatic capacitance (Section 4.11), which is directly proportional to the density of states (DOS) of the 
pertinent nanostructures. The series addition implies that the $C_{Q}$ needs to be larger than the nominal electrostatic contribution for its neglect, which would typically not be possible, as nanostructures intrinsically possess a smaller number of states indicating a smaller $C_{Q}$. We further discuss such issues in the next section.

Consequently, when the standard definition of nanostructures is modified to units where dimensionality effects or quantum mechanical considerations ought to be taken into account to fully describe their characteristics, the rationale for investigation and progress into nanostructures may be made more clear. Extant definitions in terms of small length scales (say, < $100 \mathrm{~nm}$ ) would then be inaccurate in assessing the potential of materials behavior, e.g., this may lead to ascribing unique characteristics to otherwise small structures, such as silicon nanowires, where the significant attributes are really related to reducing the length scales of investigation. Of recent studies, say over the past two decades, carbon based nanostructures, such as idealized one-dimensional carbon nanotubes (CNTs)[12] and two-dimensional graphene[13][14] have sparked much interest through their potential to change energy storage. The intrinsic interest may be traced back to the possibility of obtaining a better scientific understanding of phenomena in lower dimensions. However, the fact that any realistic material for usage must be inherently three-dimensional complicates practical understanding. As many reviews are extant in published literature of the energy storage potential of CNTs and graphene[15], we will consider later in the review, only the salient aspects of such materials relevant to charge storage.

One must then consider the deviations from assumed dimensionality, from the fundamental tenet that three-dimensional characteristics must be considered for practical usage, e.g., that the lower dimensional structures must be, at some point, connected to macroscopic 
three-dimensional contacts/loads. At one extreme, the notion of single electron levels and bands and the connection to macroscopic structures, through mesoscopic intermediates[16] must be understood. The coupling of discrete modes/channels to contacts, which can be described by a continuum of energy levels, involves consequent mixing of the energy levels/bands and is analytically difficult to comprehend.

The surface, in a quantum mechanical sense, represents the termination of a material and is composed of energy states (surface states) distinct from the bulk states due to a different environment, e.g., in terms of number of chemical bonds or due to the presence of foreign atoms from adsorbates, etc. It is then typically found that the interactions between the surface and bulk energy levels results in the formation of hybrid states, in the spirit of the Debye-Huckel model[17], distinct from either levels. For example, in a semiconductor-metal contact, the hybrid states (referred to as Tamm states) are found in the bandgap region, at one-third of the bandgap energy $\left(E_{g}\right)$ reckoned from the valence band edge[18]. It was realized that if electrons were present in the surface states there would be a proximal positive space charge region in the bulk, and the consequent formation of the thin double layer and voltage drop across the same could be critical in determining the electrochemical characteristics. The possibility of pinning of the $E_{F}$ to the defect states, where successive carrier addition negligibly affects the $E_{F}$ - until all the defect states are saturated, would be pertinent and results in band-bending and concomitant enhanced energy barrier for carrier transfer from the contact to the material. It may be surmised that in almost all cases of practical importance, that an unsymmetrical energy barrier between the external contact and the nanomaterial precludes an Ohmic contact and seamless passage of the electrical carriers (electrons/holes) from the nanomaterial to the current collector. Instead, what 
is obtained is a Schottky barrier[19] for electron and hole injection leading to a diode-like rectifying behavior. For example, the pinning of the valence band of a semiconducting nanostructure to the $E_{F}$ of a contact metal electrode would result in the description of transport in the channel of the device as through two back-to-back connected Schottky barriers (see Section 4.6.1). Only in those cases where the equivalent width of the barrier region is sufficiently small may carriers tunnel through yielding a quasi-Ohmic behavior. Such behavior has been observed, and typically for nanostructure lengths $<1 \mu \mathrm{m}$, electrical transport seems to be limited by the Schottky barriers at the metal-semiconductor interface. However, for greater lengths and in lower dimensionality, there would be a stronger influence of the defects/impurities which may induce a strong resonant backscattering and reduce carrier mobility[20]. Such defects in nanomaterials (see Section 4.8.2) would also contribute significantly to a combined capacitive and battery-like behavior, as has been found in large specific area aerogels[21] and carbon nanotubes[4,22,23] (see Section 5.2).

Aside from deeper physical implications of traversing the nanoscopic-macroscopic boundary, attention must also be paid to the methodology and placement of the nanostructures to be investigated. We consider, as an example, the case of graphene. While in popular literature, graphene has been alluded to as a planar sheet, the effective thickness is needed in estimates of the physical properties of the sheet and is subject to much debate. The single layer graphene sheet - substrate interaction, as manifested in phonon coupling [24,25] or surface assisted coupling based synthesis[26] indicates the difficulty precisely ascribing characteristics typical of a single independent sheet. Indeed, current opinion seems to indicate that the thickness of a 
graphene sheet is more clearly measurable in the bilayer form, through the height differential between the second and the first sheet.

Alternately, through considering the synthesis methodologies as well as $\pi \square \square$ orbital interactions, we are naturally led to a need for understanding the three-dimensional interactions via lower dimensional synthesis methodologies and relevant characteristics. For example, graphene synthesis has considerably evolved from the originally used "scotch-tape" methodology[27] and is often accomplished through chemical exfoliation/hydrothermal treatments[15,28] with introduction of pendant oxygen and hydrogen containing groups. (while deoxygenation using strong reducing agents such as $N$-methyl pyrillodine (NMP) has been reported[29], the C/O ratio is still substantial at 3-5[30]). Chemical Vapor deposition (CVD) based processes, which use metal substrates, for the nucleation of graphene films also typically suffer from the formation of three-dimensional graphene grains and consequent grain boundaries over large areas. Moreover, while metallic impurities may confuse the obtained characteristics from the nanocarbons, as has been extensively determined for CNTs[31] the process of transfer of the graphene films typically involves the use of relatively flexible polymeric films which may introduce additional out-of-plane interactions. It is then concluded from a reading of literature that current methodologies may not guarantee two-dimensional graphene morphology. Similar difficulties are also present for CNTs, where one-dimensional characteristics[32] may be considered more the exception than the general rule of thumb that the three dimensional/bulklike manifestation of lower dimensional nanostructures must always be a practical consideration. We will now consider such practical manifestations of charge storage and transfer through a brief review of extant devices, which accomplish charge to energy conversion. 


\section{devices: Batteries and Capacitors}

We will commence our discussion with a review of the principles underlying commonly used energy storage devices. We will focus much attention on the hybrid electrochemical capacitor (EC), which could potentially bridge and combine the fast response time and high power density of capacitors with the high energy density associated with conventional batteries. Accompanying characteristics such as a high cycle life, i.e. the capability of rapid charging and discharging, for millions of cycles, will be studied. While initial discussion will be on a review of extant materials and devices for batteries and ECs, a few examples of devices where charge storage/transfer is relevant, e.g., photoelectrochemical cells (Section 4.7) or dye-sensitized solar cells (Section 4.10) as well as alternative nanomaterials (Sections 4.8 .1 and 6.1) will be considered in later sections.

Broadly, the charge storage devices could be partitioned into batteries and capacitors as illustrative of their respective charge storage mechanisms. An energy storage device also needs to deliver energy in a given time, i.e., as codified through electrical power. This translates to the product of the voltage $(V)$ - which may also be termed as an open circuit voltage $\left(V_{O C}\right)$ through 
an equivalency to the potential difference generated across the terminals of the battery in a standalone configuration - and the current $(i)$. A battery yields power, at a constant voltage, while the charge $(Q)$ on a capacitor per unit time $(=i)$ can be discharged with a concomitant drop in voltage. In the battery, charge depleted/unit time from one electrode is recovered/unit time at the other electrode and the charge accumulation occurs at a constant/definite electrical potential relevant to the pertinent chemical reaction. Alternately, charge has to be continually built up on the two plates of a capacitor, with increasing electrical potential. Consequently, a battery would intrinsically have a larger stored energy $(=Q V)$, while a capacitor has on the average a lower energy $=1 / 2 Q V$ (with the average in the voltage from a lower limit of zero to an upper limit of $V$ volts reckoned as V/2) implying a larger energy density, almost by definition, for the former. However, as the $V$ does not need to be maintained, (say through chemical reactions as in a battery), the energy yield could occur in a faster time in the capacitor implying a higher power density. In principle, while batteries may be characterized by a capacitance they are designed to only be a minor constituent of the total energy (see Section 5.1.1) as only a small part of the total voltage would be under consideration. The above explains the fundamental dichotomy between batteries and capacitors and bridging the two device mechanisms is not readily apparent.

For obtaining the maximum voltage from a battery, it is well understood that the difference between the anodic oxidation (corresponding to electron gain) potential $\left(V_{A}\right)$ and the cathodic reduction (corresponding to electron loss) potential $\left(V_{C}\right)$ ought to be as large as possible. An interesting argument, originally due to Poynting, can be invoked to relate such an aspect to be analogous to a Carnot efficiency[33]. At a given current, $i$, the power released from the anode would be $V_{A} i$, while at the cathode, the corresponding power absorbed would be $V_{C} i$. Consequently, the difference $\left(V_{A^{-}} V_{C}\right) i=V i$, would be available for the external work. The device 
efficiency is then related to the ratio of the available energy to the input energy as equal to $\frac{V_{A}-V_{C}}{V_{A}}$, which can be related to a Carnot-like form, quite familiar to the scientific and technological community.

The maintenance (and supply) of a constant potential across a battery also implies that the causative mechanism be based on a chemical, e.g., a reduction-oxidation (redox) reaction with a fixed/constant free energy of reaction. The metal electrodes should effectively serve as infinite reservoirs/sinks of electrons (depending on whether the battery is being charged/discharged). The chemical reactions involving the ions in the electrolyte would assure a separation between the anodic and the cathodic electrochemical potentials (see Figure 1), the difference between which is manifested as the external battery voltage. When the battery is connected to a loss-less element, this would imply a constant current at a constant voltage, and the battery could work for ever, supplying and retrieving back the power= VI. However, more practically there is a dissipation of energy in an external load (of resistance, $R$ ) and the power $\left(=I^{2} R\right)$ would have to be supplied irretrievably from the battery. Other considerations of deviations from non-ideal battery performance would also need to consider the internal resistance $\left(R_{\text {int }}\right)$ of the battery, which hitherto has been assumed to be zero. However, inevitable electron transfer from (/to) the electrode to (/from) the electrolyte would yield a finite $R_{\text {int }}$. Subsequently, the voltage that can be obtained from the battery is reduced to $V-I R_{\text {int }}$, while the current is reduced to $\left(V-I R_{\text {int }}\right) /(R+$ $\left.R_{\text {int }}\right)$. The failure of the battery, say to supply the redox reaction predicted voltage over a period of time (i.e., whereby kinetic factors should be considered) would then be implicated to the increase of the $R_{\text {int }}$ which may be attributed to internal mechanisms such as loss of electrolyte 
integrity, diminished electrode performance, say through structural changes accompanying the formation of an interfacial solid-electrolyte interface (SEI) layer (see Section 4.5), etc.

Alternatively, in a capacitor, the electrodes need/do not serve as infinite sinks/reservoirs, as implied by the enhancement/loss of charge on the plates on charging/discharging. The mechanism implies that a very large voltage may be applied to charge (fill)/ discharge (deplete) the electrodes, with the upper limit being the electrical breakdown of the dielectric between the capacitor plates. The dielectric has the function of just keeping the electrodes insulated from each other and at different potentials. Redox reactions must not be allowed for they stabilize the voltage at a given thermodynamic value and yield battery like characteristics. Consequently, the the basic principle of capacitive charge/energy storage is related to the energy stored per unit volume of space, and is given by:

$$
\text { Energy }=\frac{1}{2} \varepsilon_{o} \varepsilon_{r} \xi^{2}
$$

The $\mathcal{E}_{\square} \square$ is the dielectric permittivity of free space $\left(=8.854 \cdot 10^{-12} \mathrm{C}^{2} / \mathrm{Nm}^{2}\right), \mathcal{E}_{r} \square$ is the permittivity of the medium/relative dielectric constant, storing the charge, and $\xi$ is the electric field/voltage gradient. It is then obvious that at a given voltage, the determining parameters are the $\varepsilon_{r} \square$ and the distance across which the voltage is applied (which implicates the structural influences). Such issues are further discussed in Sections 2.3.1, 2.3.2, and 2.3.3

In summary, a constant voltage generally characterizes ideal batteries over a charge/discharge period while capacitors have an increasing (/reducing) voltage when charged (/discharged). While the energy storage capacity of these devices is directly proportional to their mass, the rate at which the energy can be delivered would be related to the time constant of the 
device. A detailed discussion of the estimation of time constants, particularly relevant for capacitors in terms of the constituent impedances is outlined in Section 3.3.

\section{batteries: voltage and charge}

Several books[34] and reviews[35,36] provide a good indication of the underlying materials issues[37-40] related to battery operation and efficiency. In this section, we briefly outline the general characteristics as relevant to charge storage in these devices. Many of the pertinent principles will be illustrated with respect to Lithium ion batteries, as they constitute the largest proportion of batteries at present due to their use in portable electronic devices. The choice of Li itself is predicated on its low atomic weight potentially yielding high energy density (also see Section 2.4.1) The discussion will also be focused on $\mathrm{Li}$ ion interactions with solid materials, e.g., metal-air battery systems incorporating gas based reactions will not be considered, in spite of their widely touted superior performance as they are seem to involve formidable challenges even at a fundamental level, e.g., a knowledge of the precise electrochemical reactions involved[41]. It has also been considered in a recent review that the gain in energy density, e.g., of $\mathrm{Li} / \mathrm{O}_{2}$ cells over $\mathrm{Li}$ ion batteries would be "modest"[42].

As discussed earlier (Section 2.1), a battery operates on the thermodynamic principle that a net free energy $(\Delta G)$ and voltage $(=-\Delta G / z F)$, accompanies a redox (reduction-oxidation) reaction of the form:

$$
O+z e^{-} \underset{k_{b}}{\stackrel{k_{f}}{\rightleftharpoons}} R
$$


In the above, $O$ refers to the oxidized species which reduces (gains electrons) to $R$ - the reduced species, e.g., $\mathrm{Li}^{+}+e^{-} \rightleftharpoons L i$. The $k_{f}$ and the $k_{b}$ refer to the forward and the backward chemical rate constants, with the ratio of $k_{f}$ to $k_{b}$ designated as the overall rate constant: $k$. The reaction proceeds at a fixed thermodynamic potential, referred to as a $V_{O C}$, the open circuit potential, which for $\mathrm{Li}^{+}+e^{-} \rightleftharpoons \mathrm{Li}$ is $-3.04 \mathrm{~V}$. In this case, a positive $\Delta G$ implies that this reaction proceeds with an increase of energy and is hence not favored thermodynamically. On the contrary, $\mathrm{Li} \rightleftharpoons \mathrm{Li}^{+}+e^{-}$, yields a $V_{O C}=+3.04 \mathrm{~V}$ with a negative $\Delta G$ and is thermodynamically favored. Generally, the tendency for charge transfer so as to yield a higher $\left|V_{O C}\right|$ is related to the ease with which electrons can be transferred to and fro from the element/material. As an approximate rule of thumb, highly electropositive elements (such as $\mathrm{Li}$, $\mathrm{Ca}, \mathrm{Mg}$ ) or highly electronegative elements (such as $\mathrm{F}$ ) tend to have such a large tendency for oxidation and reduction, respectively and this is reflected through higher $V_{O C}$ values. Compounds would generally have a lower electrochemical potential $(\mu)$ - enabling use as cathodes, as charge transfer is hindered due to the necessity for charge rearrangement over a larger number of crystal units/volume, compared to elemental materials - which could consequently serve as anodes, see Figure 1, However, there does not seem to be any quantitative/direct correlation to metrics such as the electronegativity or ionization energies.

A good guide to understanding the concomitant charge transfer in a battery-like device, say for example, in an organic electrolyte constituted system is the energy level diagram - taken from [40] - and depicted in Figure 1, in which the effect of the electrode energy levels with respect to the LUMO (lowest unoccupied molecular orbital) and the HOMO (highest occupied molecular orbital) of the electrolyte is considered. The LUMO and the HOMO may correspond 
to an equivalent conduction band and the valence band of the electrolyte, respectively. The maximal $V_{O C}\left(=\left|\mu_{A}-\mu_{C}\right|\right)$ would be obtained when the electrochemical potentials of the anode and the cathode, i.e., $\mu_{A}$ and $\mu_{C}$ are perfectly aligned to the LUMO and the HOMO, respectively. In this case, charge transfer to/fro from the electrodes to the electrolyte would not occur, resulting in the total absence of internal electronic current, no internal voltage drop, and translating to an ideal voltage source. Only ionic current would be present in the electrolyte to neutralize the electrons appearing (/leaving) at (/from) the cathode (/anode) from the external circuit, i.e., positive (/negative) ions from the electrolyte moving to the cathode (/anode). However, even in such an ideal battery, the ion transfer rate across the electrodes lags the electron transfer rate, and poses a fundamental limitation in terms of the power that may be obtained. Consequently, a battery with a larger power density would have smaller ions - say, $\mathrm{H}^{+}$, as in aqueous systems. However, there is a limited voltage range of $\sim 1 \mathrm{~V}$ (see Section 2.2.3) in aqueous systems, corresponding to the elctrolysis of water.

As such matching of the energy levels across disparate materials is not possible in practice and yields a lower $V_{o c}$ as well as the formation of an interfacial layer (as in a solid electrolyte interface (see Section 4.5) - typically due to [38] parasitic transfer of electrons from the anode to the electrolyte LUMO and reduction of the electrolyte. It may be thought that the positioning of the anode/cathode chemical potentials within the electrolyte LUMO-HOMO window would preclude electron transfer from (/to) the electrode to (/from) the electrolyte and internal electronic current and voltage drop. However, when the electrolyte is used at the extremes of its voltage range (which is desirable for achieving high energy/power densities) charge transfer might yet occur at the electrode-electrolyte interface. Such considerations dictate the choice of appropriate anode and cathode materials, which would then be located above the 
LUMO and below the HOMO of the electrolyte, respectively. As a consequence of several such factors, in practical usage the voltage obtained from a battery is less than the thermodynamically predicted $V_{O C}$.

The parasitic charge transfer between the electrodes and the electrolyte is often held responsible for an induced polarization across the electrode-electrolyte interface. The consequence is a gradual reduction of the voltage output of a battery as depicted in Figure 2 . Such a kinetically induced factor diminishing the battery voltage is termed the Ohmic polarization loss[34,35] and accounts for the gradual drop - see the relatively flat part of the curve in the middle, in Figure 2, in output voltage from the postulated $V_{O C}$. At smaller and larger times, activation polarization and concentration polarization account for the relatively larger drops in $V_{O C}$. The former is related to the net energy needed for a particular redox reaction to occur considering intermediate states of a higher energy through Arrhenius activation theory. The magnitude of such activation induced polarization losses may be quite large, for example, in the use of metal-air batteries where the air-mediated kinetics may be significantly slower than for say, typical liquid-mediated kinetics. Finally, concentration polarization dominated loss occurs from limitations in the transport/diffusion of reactants to the electrodes and is typical at the end of the battery life. The kinetic limitations on charge transfer (arising from mismatched energy levels and consequent charge transfer) occur at various time scales, e.g., ranging from submilliseconds (with electron transfer reactions corresponding to chemical equilibria are rate limiting) to larger time scales (where mass diffusion is rate limiting). 


\section{Charge transfer in}

\section{solids, with emphasis on reversible intercalation}

Charge storage/release could generally be accomplished through the attachment/removal of particular charged species and may be manifested in various forms. This would entail in the main: (1) Chemical bonding based interaction - ranging from the attachment of the Li ions to moieties such as carbonyl groups/organic salts[43]to alloy formation, and (2) Intercalation, involving the reversible insertion and removal of $\mathrm{Li}$ ions, in graphite or $\mathrm{Li}$ transition metal oxide based compounds.

First, the reversible formation of Li based compounds, (e.g., fluorides[44], sulfides, oxides[45], nitrides, and hydrides) through reactions of the type: $\mathrm{Li}+\mathrm{MX}_{\mathrm{m}} \rightleftharpoons \mathrm{M}+\mathrm{LiX}$, where $\mathrm{X}=\mathrm{F}, \mathrm{O}, \mathrm{S}$, or $\mathrm{N}$, may serve for high capacity charge storage. The main idea in such $\mathrm{Li}$ conversion interactions is that more than one electron transfer may occur ( $c f$. Eqn. 2), utilizing a greater number of oxidation states of the elements in the electrodes. For example, in the case of $\mathrm{Fe}_{3} \mathrm{O}_{4}$, Li intercalation could involve 2.67 electrons per 3d-metal element[46]. Compounds such as $\mathrm{Li}_{2} \mathrm{O}$, which in the bulk are electrochemically inactive but was made reactive through the use of nanoparticles [47] were one of the first manifestations of such a proposal. While attractive in principle, the enhanced charge capacity is predictably offset through decreased reversibility and cycle life. A discussion with respect to the state of the art in terms of energy capacity through the use of such compounds is discussed later in Section 2.4.1. Extensive investigations are being conducted into halides and chalcogenide based systems based on the large degree of bonding variability, e.g., fluorides with a greater degree of ionic bonding compared to oxides or sulfides would have larger electrochemical potentials[8]. However, such bond formation like processes 
involve chemical interactions and would be susceptible to polarization effects (implying differences between the anodic and cathodic reaction potentials) as well as rate and cycling related issues related to the degree of reversibility. The consequent poor electrode kinetics, resulting directly from the low energy efficiency of the conversion reaction remains a major issue and may even be intrinsic to the particular charge storage mechanism. The Li storage compound may also be formed through a succession of intermediate phases, and hence preclude flat voltage profiles on charge/discharge (e.g., due to charging at the interfaces of the phase). The degree of electronic conductivity as well as the ionic mobility of the compound reacting with the $\mathrm{Li}$ seems to be important as it was found that $\mathrm{RuO}_{2}$ (with a resistivity of $\sim 50 \square \Omega \mathrm{cm}$ [48]) had a vastly improved Coulombic efficiency (the ratio of the charge to the discharge capacity) of around $98 \%$ and [49], in comparison to other conversion based compounds, with smaller efficiencies ranging $\sim 75 \%$.

The transfer of ions from the electrolyte bulk into the electrode bulk broadly corresponds to maximal efficiency of charge transfer and interaction and underlies its usage for rechargeable batteries. Mobile ions from the electrolyte would then move into/out of these electrode spaces while maintaining the bonding and preserving the bulk character. While such intercalation based mechanisms have been the most widely used in present day batteries, such topotactic interactions (with minimal lattice ion exchange[50]) of the $\mathrm{Li}$ ions with the host were originally though to involve minimal perturbation of the host lattice through relatively few exchange of electrons - typically of the order of 1 (see Eqn. 2). Such interactions also yield relatively high cycling efficiencies due to the possible reversibility. Considering the previously mentioned issues with chemical reaction based schemes, we focus on reversible intercalation principles, which have been used in a large variety of battery systems, e.g., for the Ni/Metal 
hydride systems intercalating $\mathrm{H}^{+}$ions (as used in the Prius car models[36]) to Li oxide cathode/graphite anode based batteries that power electrical vehicles as well as portable electronics.

It was previously discussed that while elemental forms are easier to affect charge transfer, practical issues related to the stability (i.e., elements with a large $V_{o c}$ are quite reactive with the environment) as well as the necessity for the equality of the $\mu$ with respect to the electrolyte energy levels (Figure 1) dictates the investigation of compounds for electrodes. Transition metals such as $\mathrm{Fe}, \mathrm{Co}, \mathrm{Ni}$, or $\mathrm{Mn}$ are generally chosen as the basis for the compounds for $\mathrm{Li}$ intercalation based on their high valence configurations, i.e., the implication of a conversion from $\mathrm{Mn}$ to $\mathrm{Mn}^{4+}$ in the parent compound is that four $\mathrm{Li}^{+}$ions can be accomodated and that four electrons/mole can be transferred. While several electrode materials based on such general principles are feasible (e.g., that $\mathrm{Ni}$ rich material would have high capacity at the expense of a poor cycling life and stability compared to $\mathrm{Mn}$ rich oxides), practical considerations related to cost (e.g., that of $\mathrm{Co}$ ) and long-term stability dictate the usage of a particular Li based compound for the host lattice.

Charge storage through intercalation in Li-ion batteries (Figure 3) is based on a "rockingchair" concept - where the $\mathrm{Li}^{+}$ions shuttle to and from the electrodes (constituting the ionic current within the electrolyte, which is typically a $\mathrm{Li}$ compound - such as $\mathrm{LiPF}_{6}$ with a high ionic conductivity - dissolved in a mixture of alkyl carbonates or polymers - see Section 2.2.3 for more detail). As the anode (being the supplier of electrons) needs to have a high electrochemical potential, elements such as carbon (say, in the form of graphite) are suitable. Pertinently, graphite is comprised of graphene layers separated by $\sim 0.34 \mathrm{~nm}$, and can easily accommodate the $\mathrm{Li}^{+}$ 
ions (with radius $\sim 0.07 \mathrm{~nm}$ ). Layered elements and compounds, where discrete entities are loosely bonded (say, through van der Waals bonding) are in vogue for the electrodes due to the flexibility for inserting $\mathrm{Li}$.

By way of summary, the anodic reaction on discharge as presently implemented in most Lithium ion batteries involves:

$$
z \mathrm{Li} \rightleftharpoons z \mathrm{Li}^{+}+z \mathrm{e}^{-}
$$

The $\mathrm{Li}^{+}$ions are released into the electrolyte and travel in the electrolyte towards the cathode while the electrons $\left(e^{-}\right)$traverse in an opposite sense through the external circuit/the load contributing towards a compensating cathode reaction on discharge. For a typical Lithium deficit oxide cathode of the form: $\mathrm{Li}_{1-\mathrm{n}} \mathrm{XO}_{2}$, this would involve intercalation of the $\mathrm{Li}^{+}$into the cathode through:

$$
\mathrm{Li}_{1-\mathrm{z}} \mathrm{XO}_{2}+z \mathrm{Li}^{+}+z \mathrm{e}^{-} \rightleftharpoons \mathrm{Li}_{\mathrm{z}} \mathrm{XO}_{2}
$$

Generally, on the cathode side, layered/open Lithium compounds with a lower electrochemical potential are desirable which implies the use of various compounds such as oxides, chalcogenides (e.g., sulfides), etc. While chalcogenides were invoked at the beginning of Li ion battery development in the 1970's[39] later developments shifted to oxides due to their greater ionic character. The corresponding anodic and cathodic reactions on charging involve a reverse movement of the $\mathrm{Li}^{+}$ions de-intercalating from the cathode and moving to the anode, where the corresponding cathodic reaction on charge, would be:

$$
\mathrm{Li}_{\mathrm{z}} \mathrm{XO}_{2} \rightleftharpoons \mathrm{Li}_{1-\mathrm{z}} \mathrm{XO}_{2}+z \mathrm{Li}^{+}+z \mathrm{e}^{-}
$$


This would be accompanied by an anodic reaction on charge, involving $\mathrm{Li}^{+}$intercalation within the graphite anode, thus:

$$
z \mathrm{Li}^{+}+6 \mathrm{C}+z \mathrm{e}^{-} \rightleftharpoons \mathrm{Li}_{\mathrm{z}} \mathrm{C}_{6}
$$

It is interesting to note that the anode polarity may either be positive (/negative) - during charging (/discharging). A similar notion applies to the variation of the cathode polarity. The charge neutrality also implies a variation in the oxidation state of the element $\mathrm{X}$ in the cathode, e.g., a decrease or an increase in the net positive charge would be obtained when $\mathrm{Li}^{+}$is inserted or removed, respectively. While it would be obvious that a high $n$ would be desirable for charge storage, motivating the need for multi-electron transfer systems[45,47], it has been indicated (see later discussion) that the above anodic and cathodic reactions would be reversible for $0<z$ $<0.5$ for $\mathrm{Li}_{\mathrm{n}} \mathrm{XO}_{2}$ and $0<z<1$ for $\mathrm{Li}_{n} \mathrm{C}_{6}$. The limits of $n$ correspond to the fully charged/discharged state and impute powerful oxidizing and reducing character to the electrodes, and which would directly impact the electrolyte stability (see Section 2.2.3), e.g., through the formation of parasitic electrolyte products or the evolution of gases, which are often exothermic (heat evolving). The enhanced electrical resistance in the former case may further exacerbate the thermal runaway in the battery and may even lead to explosions!

The typical charging and discharging rates, for commercial lithium ion batteries, have been reported to be in the $0.2 C$ to $1 C$ current rate, indicating that the full capacity of the cell would be stored or utilized in the 5 hour- 1 hour range, respectively[45]. While it would be desirable to accomplish quicker $C$ rates (e.g., say in charging of portable electronics with $10 C$ rates where the charging would be complete in 6 minutes), the plating of $\mathrm{Li}$ on the graphite anode with subsequent dendrites bridging the two electrodes is a well-recognized possibility and 
may lead to a short-circuiting and catastrophic failure of the battery. The likelihood of such $\mathrm{Li}$ plating may be diminished through the formation of a stable solid electrolyte interface (SEI) layer on the anode (see Section 4.5 for a more detailed discussion).

\subsection{2}

\section{Electrode Materials}

While the electrodes are generally chosen for their compatibility with the electrolyte (see Figure 1), changes in the $\mu$ of the electrode materials could be manipulated through the use or addition of elements that are relatively electropositive or electronegative with respect to a given host lattice. Through the consideration of the Pauling electronegativity (EN) scales this may, qualitatively, account for the decreasing electrochemical potentials of $\mathrm{Li}_{1-\mathrm{x}} \mathrm{XO}_{2}$ based cathodes posited for $\mathrm{Li}$ ion batteries, in the order, with $\mathrm{X}=\mathrm{Ni}(\mathrm{EN}=1.90)$, Co $(\mathrm{EN}=1.88), \mathrm{Mn}$ ( $\mathrm{EN}=1.55)$, and $\mathrm{Ti}(\mathrm{EN}=1.54)$. However, detailed crystal structure considerations would also be important.

To illustrate the principles, consider a normal spinel of the form $\left(\mathrm{B}^{2+}{ }_{1} \mathrm{~B}^{3+}{ }_{2}\right)\left(\mathrm{O}^{2-}\right)_{4}$, where $\mathrm{B}(=\mathrm{Mn}$ or $\mathrm{Fe})$ with the $\mathrm{B}^{2+}$ in the tetrahedral $(\mathrm{T})$ sites and the $\mathrm{B}^{3+}$ in the octahedral $(\mathrm{O})$ sites. In the consequently formed $\mathrm{Mn}_{3} \mathrm{O}_{4}$ or $\mathrm{Fe}_{3} \mathrm{O}_{4}, 1 / 8^{\text {th }}$ of the eight T-sites and the $1 / 2$ of the four O-sites are occupied. It was found that $\mathrm{Li}^{+}$ion insertion/removal could occur into such structures with minimal impact on the native structure[51]. There are two aspects to the $\mathrm{Li}^{+}$insertion, related to (a) structural, and (b) charge ordering. It may be expected that $\mathrm{Li}^{+}$would first be inserted into the T-sites - due to their greater number, and subsequent $\mathrm{Li}^{+}$addition would induce a shift of the $\mathrm{Li}^{+}$ ions from the $\mathrm{T}$ - sites to the $\mathrm{O}$ - sites (induced through mutual Coulomb repulsions between the $\mathrm{Li}^{+}$ions). When the $\mathrm{T}$-sites are occupied, to preserve charge neutrality, the redox state of the 
$\mathrm{Mn}^{2+}$ (with the electronic configuration of $[\mathrm{Ar}] 3 \mathrm{~d}^{5}$ ) ions would tend towards the formation of $\mathrm{Mn}^{3+}:[\mathrm{Ar}] 3 \mathrm{~d}^{4}$, with the addition of $\mathrm{Li}^{+}$. Similarly when the $\mathrm{Li}^{+}$ions are on the O-sites, the relevant redox couple would concern the $\mathrm{Mn}^{3+} / \mathrm{Mn}^{4+}$ states. When the $\mathrm{Mn}$ ions are in a high-spin state[52], the $\mathrm{Mn}^{3+}$ and $\mathrm{Mn}^{4+}\left([\mathrm{Ar}] 3 \mathrm{~d}^{3}\right)$ states are distinct, through ligand-field splitting and lifting of the degeneracy of the $t_{2 g}$ (constituted from the $d_{x y}, d_{x z}$ and $d_{y z}$ orbitals) and $e_{g}$ (constituted from the $d_{x-y}^{2}{ }^{2}$ and $d_{z}^{2}$ orbitals) manifolds, by a crystal field energy of $\sim 1 \mathrm{eV}$, which is then manifested through a reduced potential of $1 \mathrm{~V}-$ see Figure 4 below. The limit to $\mathrm{Li}^{+}$ insertion occurs when all of the $\mathrm{Mn}$ in the structure reduced to the $\mathrm{Mn}^{4+}$ form yielding $\mathrm{MnO}_{2}$. A similar reduction occurs even in acidic media (with a high $\mathrm{H}^{+}$concentration) and is more rapid with smaller particles due to the greater magnitude of the voltage gradient/electric field driving the ions[51].

However, parasitic reactions such as $\mathrm{Mn}^{3+}$ disproportionation (whereby $2 \mathrm{Mn}^{3+} \rightleftharpoons \mathrm{Mn}^{2+}+$ $\mathrm{Mn}^{4+}$ ) or the $\mathrm{Li}^{+}$ion induced structural instabilities (both due to the site occupancy as well as the Jahn-Teller distortions related to the $\mathrm{Mn}^{3+} / \mathrm{Mn}^{4+}$ changes) necessitates the use of other redox couples where such distortions are absent, such as the $\mathrm{Fe}^{2+} / \mathrm{Fe}^{3+}$, as in the olivine crystal structures of the $\mathrm{LiFePO}_{4}$ variety. Analogous to spinels (except that the oxygen anions are arranged in a hexagonal network in contrast to the cubic arrangement in spinels), such materials allow for a larger $V_{O C}$ due to the facility with which the $\mathrm{Fe}^{2+} / \mathrm{Fe}^{3+}$ redox couple voltage can be altered. Alternately, the substitution of lower valence ions (such as $\mathrm{Ni}^{2+}$ ) for $\mathrm{Mn}^{3+}$ has the effect of increasing the overall $\mathrm{Mn}$ oxidation state and reducing the propensity for $\mathrm{Mn}$ disproportionation as well as Jahn-Teller like distortions. Nevertheless, it has been suggested[51] that the crystal field stabilization energy associated with the distortion can produce a two-phase electrode, with the concomitant advantage of a flat voltage response on charge/discharge. Novel 
designs incorporating a gradation in transition metal content - through a core/shell design, e.g., a hybrid material consisting of $\mathrm{Ni}$ rich core (constituted from $\mathrm{Li}\left[\mathrm{Ni}_{0.8} \mathrm{Co}_{0.1} \mathrm{Mn}_{0.1}\right] \mathrm{O}_{2}$ ) and a $\mathrm{Mn}$ rich shell (constituted from $\mathrm{Li}\left[\mathrm{Ni}_{0.46} \mathrm{Co}_{0.23} \mathrm{Mn}_{0.31}\right] \mathrm{O}_{2}$ ) with a gradient in the $\mathrm{Ni}$ composition from the core to the shell, has been proposed to harness multiple desirable attributes[53].

The polyanions (e.g., $\mathrm{XO}_{4}$, with $\mathrm{X}=\mathrm{S}, \mathrm{P}$, or Ti), replacing the oxide ions in the olivines may enhance the binding energy and the associated $V_{O C}$ in addition to opening up the structure due to their larger size. For example, it was found, through monitoring the redox energy associated with $\mathrm{Li}$ extraction from the lithiated parent compounds, that the $\mathrm{Fe}^{2+} / \mathrm{Fe}^{3+}$ redox energy was $\sim 3.5 \mathrm{eV}$ below the Fermi level of lithium in $\mathrm{Li}_{1-\mathrm{x}} \mathrm{FePO}_{4}$ and $\mathrm{Li}_{\mathrm{x}} \mathrm{Fe}_{2}\left(\mathrm{SO}_{4}\right)_{3}$ while it was $\sim 2.8 \mathrm{eV}$ for $\mathrm{Li}_{3+\mathrm{x}} \mathrm{Fe}_{2}\left(\mathrm{PO}_{4}\right)_{3}$ [54]. The smaller value, by $\sim 0.7 \mathrm{eV}$, for the compounds with the $\left(\mathrm{PO}_{4}\right)_{3}$ poly-anion was ascribed to a lower degree of covalent bonding. An inductive effect, related to electronegativity based charge transfer, e.g., the stronger bonding strength of the S-O bond compared to the P-O bond was thought to be responsible for the higher Fermi energy of the $\left(\mathrm{SO}_{4}\right)_{3}$ constituted moieties. Similarly, a counter-cation can, via an inductive effect on the primary transition metal cation, may be used to tune the intrinsic voltage levels. There has also been considerable effort aimed at harnessing more than one Li ion per formula unit, through the use of compounds such as $\mathrm{Li}_{2} \mathrm{MSiO}_{4}$ (where $\mathrm{M}=\mathrm{Mn}, \mathrm{Fe}, \mathrm{Co}, \mathrm{Ni}$, etc.). However, in addition to the structural stability issues (as more Li has to be accommodated both in the bulk and due to surface reactions) lower voltages may also result due to the enhanced electropositive character[38,40].

In summary, the limits to the anodic and the cathodic voltages of a solid electrode can be related to the energy of the bottom of the (cation based) conduction band and the top of the 
(anion based) valence band. Such intrinsic limits are characteristic to the electrode materials and should be compared to the LUMO and HOMO of the electrolyte, respectively. The relative movement of the Fermi energy, e.g., a downward movement due to increasing charge of the elements or counter-ions, or enhanced covalent bonding would determine the obtainable $V_{O C}$. Such considerations enable us to understand qualitatively the relative magnitude of the $V_{O C}, e . g$, why the values for sulfides are lower than that of oxides. However, quantitative understanding needs the calculation of the band structure and the concomitant density of states all determine the relative suitability of materials. An examination of the band structures may indicate the basis for obtaining a larger $V_{O C}$ as is shown for a few select examples in Figure 5.

Additional insights may also be obtained through considering the cations and the anions separately. When the redox couple energy (as given by the $E_{F}$ ) interacts with the top of the anion band (constituted from the $p$-orbitals as illustrated in Figure 6), the relative positioning dictates the energy levels, e.g., the bonding and anti-bonding levels which may be formed due to redox energy level - valence band interactions and hence the carriers involved in the charge transfer. Case (b) is interesting in that both electrons $\left(e^{-}\right)$and holes $\left(h^{+}\right)$could be involved (as the formed bonding and anti-bonding orbitals are both in the anion band) implying that the intercalating ions can access two redox couples, i.e., corresponding to $\mathrm{Ni}^{3+}+e^{-} \rightleftharpoons \mathrm{Ni}^{3+}$ as well as $\mathrm{Ni}^{3+}+h^{+} \rightleftharpoons$ $\mathrm{Ni}^{4+}$. The pinning of the redox couple to a band has the desirable consequence that a change of the oxidation state, say from $\mathrm{Ni}^{3+} / \mathrm{Ni}^{4+}$ to $\mathrm{Ni}^{2+} / \mathrm{Ni}^{3+}$ - due to $\mathrm{Li}$ insertion/removal, does not change the Fermi energy and a relatively constant potential is maintained on charge and discharge cycling. Additional interactions due to elemental substitutions, e.g., Ni substituting for 
$\mathrm{Mn}$ in $\mathrm{Li}_{1-\mathrm{x}}\left(\mathrm{NI}_{0.5} \mathrm{Mn}_{0.5}\right) \mathrm{O}_{2}$, can also serve to raise the $\mathrm{Ni}^{3+} / \mathrm{Ni}^{4+}$ redox couple (relative to the anion $2 \mathrm{p}$ bands) and harness the voltage specific to the couple.

2.2.3

The influence of the

\section{electrolyte/ion-transfer medium}

It is previously noted that the maximum $V_{O C}$ obtainable from a battery is directly related to the energy gap (the LUMO - HOMO separation) of the electrolyte. Additional requirements for the electrolyte [40] include (i) a large ionic conductivity $\left(>10^{-4} \Omega^{\square} \mathrm{cm}^{-1}\right)$ for the redox species (e.g., $\mathrm{Li}^{+}$in $\mathrm{Li}$ ion batteries), coupled with a (ii) small electronic conductivity $\left(<10^{-10}\right.$ $\Omega^{\square} \mathrm{cm}^{-1}$ ), (iii) intrinsic and extrinsic (with respect to the electrode) chemical and temperature stability, and (iv) ability to form and maintain a stable SEI (Section 4.5) to preclude electron transfer to the electrolyte from the electrodes. Considerations related to cost, toxicity and environmental stability are of course important and preclude the widespread usage of otherwise suitable electrolytes such as ionic liquids (Section 2.3.3).

The possible electrolyte systems for batteries are then electronic insulators while being ionic conductors and range from aqueous systems, organic liquids (such as ethylene/methylene carbonates) to ionic liquids and polymers. The aqueous systems have been extensively used (e.g., in the $\mathrm{Pb}$ acid battery or the Ni-metal hydride systems) due to their lower electrical resistance and reduced flammability. Additionally, from an electrochemical point of view, in aqueous systems the oxygen can participate in both cathodic and anodic reactions, viz., $4 \mathrm{OH}^{-} \rightarrow \mathrm{O}_{2}+$ $2 \mathrm{H}_{2} \mathrm{O}+4 \mathrm{e}^{-}$(at the anode) and $\mathrm{O}_{2}+2 \mathrm{H}_{2} \mathrm{O}+4 \mathrm{e}^{-} \rightarrow 4 \mathrm{OH}^{-}$(at the cathode) and serves an important function of a shuttling species balancing the overall cell as through providing some measure of 
overcharge protection[36]. However, a major disadvantage of using an aqueous system is that the maximum voltage that could be obtained is restricted to a thermodynamic limit of $\sim 1.2 \mathrm{~V}$ (corresponding to the difference of standard electrode potentials between the reactions: $4 \mathrm{OH}^{-} \rightarrow$ $\mathrm{O}_{2}+2 \mathrm{H}_{2} \mathrm{O}+4 \mathrm{e}^{-}$of $-0.4 \mathrm{~V}$ and $4 \mathrm{H}_{2} \mathrm{O}+4 \mathrm{e}^{-} \rightarrow 2 \mathrm{H}_{2}+4 \mathrm{OH}^{-}$of $-1.6 \mathrm{~V}$, all potentials considered with respect to the normal hydrogen electrode) and necessitates the exploration of alternative electrolyte systems.

Organic liquid electrolytes (incorporating Li salts such as $\mathrm{LiPF}_{6}$ combined with carbonate blends, e.g., ethylene/methylene carbonates) can be synthesized with energy gaps $\sim 3.5 \mathrm{~V}$, are in extensive use. Moreover, the establishment of a solid-electrolyte interface is crucial (see Section 4.5) and the electrolytes are chosen such that an electrically insulating layer forms at the interface to preclude/minimize electrode-electrolyte interactions. Considering the typical incompatibility of the $E_{F}$ of the electrodes with the LUMO/HOMO levels of the electrolyte, common electrodes (e.g., the graphite anode) employ the SEI layer through the use of appropriate electrolytes (e.g., ethylene carbonate in the case of graphite electrodes), which should ideally be porous to the intercalating ions. However, since the interface necessarily is constituted of and consumes the electrode and the electrolyte material, it corresponds to wastage of material. However, charge could nevertheless be stored in the interface and may contribute to the electrical capacitance.

Under steady-state current flowing conditions, the concentration profiles of the constituent $\mathrm{Li}^{+}$and $\mathrm{PF}_{6}^{-}$ions are linear (see Section 4.4 and Figure 26) for the significance of such a relation). The transference number of the $\mathrm{Li}^{+}$was found to be a prime determinant of the ionic conductivity characteristics[55]. Under dynamic conditions, corresponding to current 
switching on/off, the ionic current is predominantly through migration (/drift) of both the anions and cations and steady state conditions are reached leading to the establishment of a concentration profile when the drift and diffusion contributions are equal (Section 4.4). However, flammability issues coupled with electrolyte decomposition and sensitivity to moisture have resulted in a search for organic electrolyte alternatives. A solid electrolyte is often preferred for compactness and safety considerations and includes solvent free systems such as PEO (polyethylene oxide) containing $\mathrm{Li}$ salts such as $\mathrm{LiPF}_{6}$ or $\mathrm{LiAsF}_{6}$. However, the downside is a lower ionic conductivity $\left(<10^{-5} \Omega^{\square} \mathrm{cm}^{-1}\right.$ ) Several efforts to enhance the conductivity through the use of nanoscale fillers such as carbon nanotubes have been deployed, where it has been proposed that heterogeneous doping[56,57] through which the nanotubes may preferentially adsorb anions from the $\mathrm{Li}$ salt creating anion vacancies may enhance the $\mathrm{Li}^{+}$mobility.

Polymeric materials are advantageous for solid-state electrolytes in that they offer an all solid-state version with the possibility of higher energy density. Moreover, they are typically safer than liquid based systems and are economical to produce. A major issue is the relatively low ionic conductivity (e.g., of the order of $10^{-7} \Omega^{\square} \mathrm{cm}^{-1}$ at room temperature, for $\mathrm{PEO}_{6}: \mathrm{LiSbF}_{6}$ ). In efforts to improve the conductivity, first, an understanding of the basic conduction mechanisms needed to be obtained. Given that polymers typically consist of both crystalline and amorphous regions[58], it was thought that the loosely fitting, dynamic, amorphous phases dominated the conduction above the glass transition temperature $\left(T_{g}\right)$. The local motion of the polymer chains creates new coordination sites facilitating ion motion, as was also confirmed through NMR (Nuclear Magnetic Resonance) studies. The relatively slow rate at which processes occur was then thought to be a major obstacle for improving polymer 
conductivity to values greater than $10^{-7} \Omega^{\square} \mathrm{cm}^{-1}$. However, it was discovered[59] - through the preparation of pure crystalline and amorphous phases of $\mathrm{PEO}_{6}: \mathrm{LiPF}_{6}$, that the crystalline phase also contributes or even dominates the ion motion, with a preference towards cation mobility. The NMR lineshape was used as an indicator of the ion dynamics and it was seen that while the linewidth of the anions (say, P) was relatively constant with temperature, there was a significant narrowing for the $\mathrm{Li}^{+}$. The relative lack of movement of the $\mathrm{P}$ with respect to the $\mathrm{Li}^{+}$cations was used to infer the larger mobility of the latter, and rationalized through the ready availability of deterministic sites. The consequent lowering of the local entropy and energy increases the rate of ion hopping. The ion movement was also found to increase with dimensionality due to the increased number of pathways. Similar to ionic solids (e.g., ceramic materials) [60], the presence of defects, such as vacancies or interstitials was also considered advantageous for substantial increment in conductivity. A further improvement in the conductivity of $\mathrm{PEO}_{6}: \mathrm{LiXF}_{6}(\mathrm{X}=\mathrm{P}, \mathrm{As}$, $\mathrm{Sb}$ ) compounds, by thirty-fold, was predicated on the substitution of the $\mathrm{XF}_{6}^{-}$ions, to the extent of $5 \mathrm{~mol} \%$, with isovalent $\mathrm{N}\left(\mathrm{SO}_{2} \mathrm{CF}_{3}\right)_{2}{ }^{-}$ions[61]. The difference in the size, shape, and charge distribution of the bulkier anions compared to the host lattice anions was thought to modulate the potential energy of the $\mathrm{Li}^{+}$ions and enhance the cation mobility. One such mechanism involves the elastic deformation of the lattice through such substitution which results in a lattice "loosening" [62]. The lowering of the melting point as well as a lowered enthalpy for the formation of defects would be the result. Such a mechanism seems to have been invoked first for ionic solids, e.g., AgI-AgBr system[62], where again the substitution of higher radius I- for the lower radius $\mathrm{Br}^{-}$resulted in a doubling of the ionic conductivity. Consequently, defects could be produced in ceramics and now even polymeric materials, through elastic considerations at ionic sites in the lattice. While the defect density (also see Section 4.8.2), which may contribute to the 
ionic conductivity may be increased through such methods, it would be pertinent to (i) conclusively establish the extent to which the ionic conductivity is enhanced for a given dopant isovalent ion, and (ii) examine the reproducibility of the enhancement for a given doping content. It was noted that the limit could be set by whether the crystallinity could be maintained, e.g., beyond a certain doping there was on onset of the amorphous phase. The typical ionic/polymer conductivity variation with temperature, for a crystalline phase, could be fit through an Arrhenius theory based principle, i.e. the conductivity $(\sigma)$ would vary exponentially with temperature $(T)$, through a relation of the form: $\sigma \sim \exp \left(-E_{a} / k_{B} T\right)$ with $E_{a}$ being an activation energy involving the mobile ion/defect (vacancy or interstitial). However, the formation of an amorphous phase, on isovalent ion substitution, was diagnosed through a curvature in the linear (expected from the Arrhenius theory) $\ln (\sigma)-T$ line and fit better to a Vogel-Tamman-FulcherHesse (VTFH) expression of the form: $\sigma \sim T^{1 / 2} \exp \left(-A /\left(T-T_{o}\right)\right)$, suggestive of viscous correlations and polymer chain movement[63].

Increased ionic conductivities $\left(>10^{-3} \Omega \mathrm{cm}^{-1}\right.$ ) have also been achieved in LiI based composites, infiltrated with nanoporous $\mathrm{Al}_{2} \mathrm{O}_{3} / \mathrm{SiO}_{2}$ through such charge transfer mechanisms, where the interaction of the space charge regions of the nanoparticles confers increased mobility in the layers and hence the composite[64]. A substantial electric field is relevant to a space charge layer (see Section 4.6.1), i.e., through depletion of a particularity polarity of charge carriers, and provides a driving force for particular ions. The formation of such layers to drive $\mathrm{Li}^{+}$ions has been a major engineering imperative for enhanced electrolyte conductivity. An interesting case where interfaces may accumulate charge concerns $\mathrm{Li}$ storage in a $\mathrm{RuO}_{2}-$ based 
$\mathrm{Li}$ ion battery, involving the presence of $\mathrm{Li}_{2} \mathrm{O} / \mathrm{Ru}$ composites. While $\mathrm{Li}_{2} \mathrm{O}$ is considered a poor electronic conductor, $\mathrm{Ru}$ is unable to accommodate the $\mathrm{Li}^{+}$ions. However, there might yet be a significant uptake of $\mathrm{Li}$ at the $\mathrm{Li}_{2} \mathrm{O} / \mathrm{Ru}$ interface by way of "job sharing " [64] between the two individual phases. The confinement of ions in very narrow interfacial layers may also induce an energy re-distribution in analogy to more traditional electron confinement effects.

Consequently, a lack of robustness related to the electronic/ionic conductivity ratio, as well as the environmental stability (e.g., sensitivity to moisture or temperature excursions) has hitherto limited the use of solid-state electrolytes or ionic liquids. The lack of a shuttle species in the organic and ionic liquid electrolytes, as in the aqueous systems, is a major issue in the stability whereby cathodic and anodic reactions have to be individually and carefully monitored. The role of the electrolyte is further exacerbated in proposed high capacity $\mathrm{Li} / \mathrm{S}$ or $\mathrm{Li} / \mathrm{O}_{2}$ batteries (See Section 2.4.2), which have been touted to have energy densities approaching $12,000 \mathrm{Wh} / \mathrm{kg}$ ! The need for excess $\mathrm{Li}$ at the anode and contaminant free $\mathrm{O}_{2}$ at the cathode has been a major obstacle in the achievement of such high densities. Concomitantly, in such systems, the electrolyte must serve multiple functions, i.e., (i) serve as an efficient $\mathrm{Li}^{+}$conductor, and (ii) be stable to the $\mathrm{S} / \mathrm{O}_{2}$ through allowing their diffusion through, while not interacting with the resultant sulfides or oxides (which would reduce the electrode capacity), in addition to restrictions on volatility and safety. Consequently, traditional $\mathrm{LiPF}_{6}$ :carbonate based electrolytes were deemed to be unsuitable due to the interaction with the reduced $\mathrm{O}_{2}$ species leading to parasitic Li carbonate compounds, as well as the production of electrode contaminating $\mathrm{CO}_{2}$ and water, all of which serve to degrade the electrolyte[65] rather than assist in the formation of the expected cathode product (i.e., $\mathrm{Li}_{2} \mathrm{O}_{2}$ ). Alternate ether based electrolyte systems, which may be more stable compared to the organic carbonates, such as tetraglyme, dimethoxyethane: DME, 
1,3-dioxolane, polyethylene oxide: PEO, etc. have been studied[66]. A large oxidation potential with respect to $\mathrm{Li} / \mathrm{Li}^{+}$(of $\sim 4.5 \mathrm{~V}$ ) in addition to low volatility (due to their high molecular weights), as well as low cost, were all desirable attributes. However, while they seem to be more stable compared to the organic electrolytes, decomposition of the electrolyte still occurs concomitant with the formation of $\mathrm{Li}_{2} \mathrm{O}_{2}$ and the decomposition products accumulate with cycling leading to a reduced capacity. It was then concluded that there are hundreds of pathways for the electrochemical reactions, in a $\mathrm{Li} / \mathrm{O}_{2}$ cell, making it "virtually impossible" [65] to formulate a unique reaction path, implying a paradoxical situation where the species (i.e., $\mathrm{O}_{2}$ ) that yields high energy density does so through its extreme reactivity. The search is then still on for optimal electrolytes for $\mathrm{Li} / \mathrm{O}_{2}$ and $\mathrm{Li} / \mathrm{S}$ battery related systems.

Concluding the survey on battery related materials, we will now proceed to review the other major category of charge storage, an electrochemical capacitor (EC) which has the virtue of quick energy delivery.

\section{Electrochemical}

\section{capacitors}

Generally while batteries have received much attention due to their widespread usage as well as frequent need for replacement, electrochemical capacitors (ECs) are noted for their reliability and have been quietly and extensively used. Their applications range from popular power tools such as cordless electric screwdrivers [1]/cutters[2], to energy management/conservation applications, e.g., during elevator operation, energy could be stored during the downward descent and released for upward ascent[1,67], and energy storage, e.g., to 
store off-peak electricity from the utility grid at night. In many cases, the ECs could be used in concert with conventional battery systems. For example, in automobile applications a short burst of high speed would be available from the capacitor, while motive energy for steady longer duration distance coverage would be the advantage offered by a battery source.

In an EC, the charge/energy storage harnesses the mechanisms intrinsic to (a) electrical capacitors, where the potential difference between charges of opposite polarities is a measure of the electrical energy stored, and (b) electrochemical activity, intrinsic to chemical reactions occurring at the surfaces of electrodes which again results in a net potential difference and energy storage. To illustrate (Figure 7), when a positive/negative electrode (e.g., the anode/cathode) is inserted into an electrolyte (liquid/solid), charges of opposite polarity are attracted and form an electrical double layer with an associated capacitance (the double layer capacitance: $C_{d l}$ ). Concomitantly, the potential dependence of the coverage of adsorbed electroactive species may be related to redox chemical processes. The consequent charge transfer then contributes to a Faradaic capacitance $\left(C_{F}\right)$ or a pseudo-capacitance: $C_{p}$. Due to the nature of charge storage, $C_{d l}$ can be discharged and charged rapidly and contributes to high power density while $C_{F} / C_{p}$ mimics battery like behavior and would increase the energy density. Proper optimization of either component can help ECs span a greater range of energy and power densities. A combination of all such capacitances yields a net measured capacitance $\left(C_{\text {meas }}\right)$, which is the final determinant of the energy capacity. 
Table I A comparison of the characteristics, performance, and cost of the state of the art electrochemical capacitors with a popular battery system

\begin{tabular}{|l|c|c|}
\hline \multicolumn{1}{|c|}{ Characteristic } & $\begin{array}{l}\text { Lithium ion } \\
\text { battery }\end{array}$ & $\begin{array}{l}\text { Electrochemical } \\
\text { capacitor }\end{array}$ \\
\hline Charge/discharge time & $\sim$ minutes & $\sim$ seconds \\
\hline Cycle life & $<5000 @ 1$ C rate & $>500,000$ \\
\hline Energy density $(\mathrm{Wh} / \mathrm{kg})$ & $50-100$ & 5 \\
\hline Power density $(\mathrm{kW} / \mathrm{kg})$ & $0.5-1$ & $5-10$ \\
\hline Cycle efficiency & $<50$ to $>90 \%$ & $<75$ to $>95 \%$ \\
\hline Cost/Wh & $\$ 1-2 / \mathrm{Wh}$ & $\$ 10-20 / \mathrm{Wh}$ \\
\hline Cost/kW & $\$ 75-150 / \mathrm{kW}$ & $\$ 25-50 / \mathrm{kW}$ \\
\hline
\end{tabular}

The working of an electrochemical capacitor is then intermediate to the two traditional types of charge storage systems, i.e., capacitors and batteries, but the dividing lines are not easily defined. Given that the $W$ scales as $C_{\text {meas }} V^{2}$, while the power density, $P$, scales as $V^{2} / R$, it is obvious that a high $C_{\text {meas }}$ over a large voltage range $(V)$ with minimal electrical resistance, $R$ (through contributions from the electrolyte, electrolyte-electrode interface, and the electrode/current collector) is desirable. Significant research has been carried out into investigating the enhancement /optimization of these parameters, e.g., through increasing the surface area through the use of porous carbon structures, with large specific surface area (of $\left.2000 \mathrm{~m}^{2} / \mathrm{g}[68]\right)$. The use of oxides, such as $\mathrm{RuO}_{2}$ [69] or $\mathrm{MnO}_{2}$ electrodes, constituted of multivalent ions, has been advocated to induce additional forms of charge storage, such as the $C_{P}$, which adds in parallel onto the nominal area based capacitance. The use of organic electrolytes or ionic liquids[70], instead of aqueous systems, could also increase cell voltages from $\sim 1 \mathrm{~V}$ to $\sim 3 \mathrm{~V}$ and $\sim 6 \mathrm{~V}$ (Section 2.3.3), respectively, with potentially an order of 
magnitude increase in $W$ and $P$. However, improving the poor electrical conductivity of the alternative electrolyte systems is still a major obstacle. However, in spite of such efforts presently used electrochemical capacitors, which are mostly based on porous graphitic carbon, typically possess energy densities of the order of $5 \mathrm{Wh} / \mathrm{kg}$ and power densities of $\sim 5-10$ $\mathrm{kW} / \mathrm{kg}[9,10]$, which is barely competitive with battery performance. To realize the full scope of ECs, the underlying reasons for the low $W$ and $P$ have to be understood and remedied. For instance, low values in $W$ arise due to insufficient capacitance, and the high $R$ intrinsic to the porous carbon limits the $P$. It is then the aim to elucidate fundamental issues relevant to optimizing EC parameters. It is also to be noted that the term "supercapacitors" has been used for ECs, and may be applicable when effects in addition to the $C_{D L}$ contribute. We will first discuss the fundamental double-layer attribute of the ECs/ultracapacitors.

2.3.1

The double layer

A fundamental attribute of an EC is that for an electrode of a given polarity, opposite charges are attracted to and stored on the electrode surface. The formation of a double layer of charge of two different polarities (see Figure 7) is reminiscent of a two parallel plate capacitor and the consequent device has been referred to as an electrical double layer capacitor (EDLC). One may also make analogies to the charging of an electrode when immersed in a plasma, e.g., an unconnected/floating electrode generally tends to get negatively charged due to the higher energy and mobility of the electrons compared to the ions[71]. Indeed the analogy of the electrolyte being represented through a plasma, in order to increase the working range of the 
electrolyte to $10 \mathrm{~V}$ has very recently been discussed under the terminology of "plasma electrochemistry"[72]. An electrode sheath consisting of a positive ions screening layer surrounds the electrons to a spatial extent corresponding to the Debye length, and consequently possessing an intrinsic capacitance. There has been extensive study focused on the understanding and accurate characterization of the double layer[73-76], especially with regards to its effective thickness. The aim has been to accurately estimate the resultant double layer capacitance $\left(C_{d l}\right)$ and correlate to the experimentally determined $C_{\text {meas }}$.

The initial efforts assumed a rigid double-layer (the Helmholtz model), where the capacitance, $C_{H}=\frac{\varepsilon_{r} \varepsilon_{o}}{d}$ with an effective thickness $(d)$ corresponding to a molecule/ion diameter. For an aqueous medium, with a bulk $\mathcal{E}_{r} \square=78$, and $d=0.2 \mathrm{~nm}, C_{H}$ would be of the order of 350 $\mu \mathrm{F} / \mathrm{cm}^{2}$. However, typical measured capacitance (i.e., the $C_{\text {meas }}$ ) is orders of magnitude smaller at $\sim 10-20 \mu \mathrm{F} / \mathrm{cm}^{2}$. Additionally, a variation of the measured capacitance with applied voltage on the electrode was observed, which seems outside the purview of the Helmholtz model. A major issue in the theoretical estimate was the assumption of the bulk dielectric constant[77] close to the electrode-electrolyte interface. For instance, the large electric field at the surface (due to the potential drop over a size scale of an ion) implies the necessity for considering orientational effects on the electrolyte constituted water molecules, in addition to enhanced polarization[78]. Consequently, the $\varepsilon_{r} \square$ may be considerably reduced to as low as 4.7 for ion-electrode distances of the order of $0.1 \mathrm{~nm}$ and to 7.8 for $0.2 \mathrm{~nm}$ (note that the radius of the $\mathrm{OH}^{-}$ion is $0.155 \mathrm{~nm}$ ). The order of magnitude reduction in the $\varepsilon_{r} \square$ (from 78 to 7.8 ) correspondingly reduces the $C_{H}$ to $\sim 35 \mu \mathrm{F} / \mathrm{cm}^{2}$. 
Additionally, there is a lack of knowledge on the precise value of the $d$. While the charge in the metal electrode is considered to be perfectly mobile, a distinction needs to be made between the counter-balancing charge from the electrolyte into (a) immobile surface charge, and (ii) mobile charge in the bulk of the electrolyte. The formation of the latter charge is driven both by spatial constraints at the surface of the electrode as well as the charge concentration gradient extending from the electrode surface outwards into the electrolyte. Instead of a rigid ionic layer, two constituent arrangements of the ions, termed the inner (IHP) and outer (OHP) Helmholtz planes need to be considered[73,79]. While the ions of the IHP are specifically adsorbed, the ions of the OHP are nonspecifically adsorbed and are screened through solvation - see Figure 8. The immobile charge is modeled as a space charge layer - Figure $8(\mathrm{~b})$, with a defined $C_{H}$ along with a $C_{D}$ representing all the other contributions from the mobile charge in the electrolyte (constituting the diffuse double layer). The charge distribution in the electrolyte is depicted in Figure $8(\mathrm{c})$, and the voltage applied on the electrode is correspondingly partitioned between the two capacitances - Figure 8(d).

The effects of diffusion and mass transfer, coupled with the randomizing effects of thermal energy $\left(=k_{B} T\right.$, where $k_{B}$ is the Boltzmann constant $=1.38 \cdot 10^{-23} \mathrm{~J} / \mathrm{K}$, and $T$ is the temperature, and is of the order of $26 \mathrm{meV}$ at room temperature) are considered through the diffuse layer, which initiates past the OHP and extends into the bulk solution. Concomitantly, the charge distribution is displaced to a greater distance from the electrode also effectively reducing the capacitance. The Gouy-Chapman theory, which is essentially the solution of the PoissonBoltzmann equation for the concentration of carriers in an electrolyte near an electrode is invoked for the estimation of such diffuse layer capacitance $\left(C_{D}\right)$. 
The Debye length $\left(L_{D}\right)$, which is typically defined through Eqn. (7) below[79,80], is a typical measure of the diffuse layer thickness, with $z$ as the ion charge $\left(e . g ., z=1\right.$, for a $\mathrm{Na}^{+}$ion, and $z=-1$, for a $\mathrm{Cl}^{-}$ion ) and $I^{o}$ as the ion species concentration in the electrolyte.

$$
L_{D}=\sqrt{\frac{\varepsilon_{r} \varepsilon_{o} k_{B} T}{2 z^{2} e^{2} I^{o}}}
$$

For a $1 \mathrm{M}$ (moles/liter) electrolyte (corresponding to the Avogadro number related $6.02 \cdot 10^{20}$ singly charged ions $/ \mathrm{cm}^{3}$ ), the $L_{D}$ is of the order of $0.3 \mathrm{~nm}$ and varies inversely with the $n^{o}$. While a further increase in the $L_{D}$ may be thought plausible from an enhanced electrolyte concentration, typical solubility limits are of the order of $10 \mathrm{M}$, implying a limit close to $\sim 0.1$ nm. The capacitance magnitude could in principle then be of the same order as the $C_{H}$. However, the Gouy-Chapman theory was also successful in indicating a potential $\left(\phi_{e}\right)$ dependence for the observed capacitance (through considering the Poisson equation for the potential variation), which could not be accommodated in the model for the $C_{H}$. A diffusion capacitance $\left(C_{D}\right)$ based on the potential variation with distance due to the ionic distribution, was derived to be of the form[79]:

$$
C_{D}=\sqrt{\frac{2 \varepsilon_{r} \varepsilon_{o} z^{2} e^{2} I^{o}}{k_{B} T}} \cosh \left(\frac{z e \phi_{e}}{k_{B} T}\right)
$$

The origin of the cosh () term can be traced to the sum of the individual effects of the activation barriers of the positive $(z>0)$ and the negative $(z<0)$ ions through $\exp \left(\frac{z \ell \phi}{k_{B} T}\right)$ terms, while its integral (with respect to the voltage) was related to the net charge through the difference 
of the exponential terms - through a sinh () dependency. However, the cosh () term in Eqn. (8), also indicates an increase of the capacitance without limit as the voltage is increased, contrary to experimental observation, where at large electrode potentials, a saturation of the capacitance was observed. Such a possible anomalous increase could then be suppressed by the series addition of the $C_{H}$ with the $C_{D}$. Such a rationale, as expressed through the Stern modification to the GouyChapman theory was that at sufficiently large applied potentials, oppositely charged ions would indeed adhere strongly to the electrode, with an average separation distance subject to limitations arising from ionic radius as well as the influence of the electrolyte (e.g., the solvation of the ions in aqueous systems). The implication is that as $n^{o}$ or $\phi_{e}$ is increased, at a given temperature, that $C_{D}>C_{H}$, and the diffusion layer (and the $C_{D}$ ) becomes increasingly irrelevant for the measured capacitance. For example, at $1 \mathrm{M}$ and with a $\phi_{e}=0.026 \mathrm{~V}\left(=k_{B} T\right.$, at room temperature $)$, and an $\mathcal{E}_{r} \square \square \square \square \square \square \square$ the $C_{D}$ is $230 \mu \mathrm{F} / \mathrm{cm}^{2}$ and increases to $2300 \mu \mathrm{F} / \mathrm{cm}^{2}$ at $\phi_{e}=3 k_{B} T$. Taken in series with the $C_{H}$ (estimated to be $\sim 35 \mu \mathrm{F} / \mathrm{cm}^{2}$ from previous discussion in this section), the net capacitance would be of the order of $\sim 30 \mu \mathrm{F} / \mathrm{cm}^{2}$ and $34.5 \mu \mathrm{F} / \mathrm{cm}^{2}$, respectively indicating a closer approach to $C_{H}$ the larger the value of the $C_{D}$.

When an assemblage of two electrodes, typical to a cell, is connected to an external circuit the capacitances from the two equivalent electrodes add in series and the net cell capacitance would be approximately half of the estimated double layer capacitances. Enhancement of the net capacitance can be obtained by placing an electrode undergoing redox reactions (indicative of Faradaic contributions, such as $C_{F}$ or the pseudocapacitance $C_{P C}$ : Section 5.1) as one of the pair of electrodes. If the $C_{F / P}$ is larger than the estimated $C_{D L}$ (which in turn is comprised of contributions from $C_{H}$ and $\left.C_{D}\right)$ a net capacitance of $\left(1 / C_{F / P C}+1 / C_{d l}\right)^{-1} \sim C_{d l}$ is 
obtained. Such hybrid battery-capacitor cells may thus be constructed. Note that replacing both the electrodes - as in a battery results in a higher redox capacitance yielding higher energy density at the expense of a lowered power density. For a further discussion of the relationship of battery and capacitor like behavior, see Section 5.1.3. It has been discussed there, that while the capacitance contribution is universal for electrochemical devices, it forms a relatively minor part of the overall energy density.

\subsection{2}

\section{Additional capacitances}

\section{modulate double layer capacitance}

The attraction of oppositely charged ions to an electrode was previously considered to be the basis of the formation of a double layer. While such a representation indicate an intuitive way of understanding the electrode-electrolyte interface, it has been well recognized that it yet represents a crude model due to its (a) lack of predictability of the measured capacitance: $C_{\text {meas }}$, as well as (b) the abrupt nature of the considered interfaces, say in between the IHP and the OHP of the Helmholtz layer and with the diffuse double layer. Additionally, the typical presence of adsorbed ions is not considered in the above model, the effects of which may be manifested in pseudocapacitance (see Section 5.1) related phenomena. As the surface of metal electrodes is typically negatively charged (due to the larger kinetic energy of the electrons in the electrode as well as in the electrolyte), cation adsorption may be preferred. However, the heavily solvated character of the cations precludes their efficient adsorption. Such electrode charging effects may be understood through surface energy measurements $[79,81]$ where ion adsorption reduces the 
interfacial surface energy and a peak corresponding to the point of zero charge (PZC) - which does not occur at zero potential due to the adsorbed ions, is exhibited.

It has also been assumed that the electrode is a perfect metal (which cannot support electric fields). However, perfect screening that occurs with metals (of carrier concentrations of $10^{29} / \mathrm{m}^{3}$ ) of the electric fields may not always be assumed, e.g., when non-metallic electrodes, such as semiconductors (see Section 4.6) or nanostructures (see Section 4.9) are used. While the rationale for the use of semiconductors, such as Si may be due to the widespread usage in the electronics industry presenting the possibility of integration with electrochemical sensors or measurement apparatus, nanostructures such as carbon nanotubes are in vogue due to the high surface area. Consequently, with lower $n$, say of the order of $5 \cdot 10^{20} / \mathrm{cm}^{3}$, the screening distance would be of the order of $0.34 \mathrm{~nm}$, which is the thickness of a graphene sheet. The implication is that an atomic layer sheet would not be able to screen the electric fields and the assumption of metallic characteristics for the electrode would not be correct. The consequence is a charge rearrangement in the electrode, where a potential gradient (due to the penetrating electric field) would yield a space-charge capacitance $\left(C_{s c}\right)$. As the potential variation in the material would be expected to be of similar origin to the $C_{D}$ (i.e., due to Poisson-Boltzmann statistics), the $C_{S C}$ may be calculated by similar means, and yields[19]:

$$
C_{S C}=\sqrt{\frac{2 \varepsilon_{r} \varepsilon_{o} z^{2} e^{2} N}{k_{B} T}} \cosh \left(\frac{z e \phi_{s}}{k_{B} T}\right)
$$

Here the $\varepsilon_{r} \square, N$, and $\phi_{s}$ refer to the dielectric constant, carrier concentration, and potential drop relevant to the solid electrode material. It is important to note that the $C_{S C}$ would be in series with the $C_{d l}$ (just like the $C_{D}$ ) and may dominate the overall capacitance if it is smaller. 
One manifestation of the $C_{s c}$ was through a consideration of the charge capacity of the basal plane of stress-annealed pyrolytic graphite, where with $\mathcal{E}_{r} \square=3$ for $n=6 \cdot 10^{18} / \mathrm{cm}^{3}$ the pre-factor for $C_{s c}$ was computed to be $\sim 4.5 \mu \mathrm{F} / \mathrm{cm}^{2}$ and was close to the value observed at $\phi_{s}=0[82]$. However, for a larger $\phi$, say even $\sim 0.2 \mathrm{~V}$, the $C_{S C}$ would be larger by three orders of magnitude with a diminished relevance for determining the overall measured capacitance.

In summary, we have discussed various capacitances, i.e., intrinsic to the electrolyte: $C_{D L}$ (constituted from $C_{H}$ and $C_{D}$ ) as well as those intrinsic to the electrode, i.e., $C_{S C}$ which all add in series. Since, the obtainable charge for an EC may be primarily determined through the $C_{H}$ (involving the least time scales and hence the largest obtainable power density) the $C_{D}$ and the $C_{S C}$ may be considered as parasitic capacitances which would reduce the capacitance, i.e., the applied potential is partitioned across the material of the electrode and the electrolyte instead of across the electrode-electrolyte interface. While the $C_{S C}$ could in principle be substantially enhanced through metallic materials, $C_{D}$ is almost always present. It is important then to note that the value of $\phi_{e}$ or the $\phi_{s}$ seems to have an enormous impact in determining the overall capacitance that may be obtained from a practical EC, with a lower value being more conducive to comparison with experimentally obtained values. However, we have not yet considered material non-idealities such as the presence of surface states (See Section 4.6.1) or even the possibility of additional reactive sites (see Section 4.8.2), which could modify the capacitances. A finite number of sites, as would be relevant to nanostructures, would be manifested in a chemical capacitance (see Section 4.10) and a quantum capacitance (see Section 4.11) as well, which are also in series with the $C_{H}$. 
The consequence for charge storage, through ECs, is then that the combination of all such factors yields a $C_{\text {meas }}$ of $\sim 10 \mu \mathrm{F} / \mathrm{cm}^{2}$, and assuming that the charge is stored almost entirely on the surface, a large capacitance/charge capacity per unit mass would be obtained by multiplying the $C_{\text {meas }}$ by a high surface area, low mass density substance, e.g., in the case of CNT based nanostructure, where the material is all surface, values of the order of $3500 \mathrm{~m}^{2} / \mathrm{g}$ have been indicated[83]. Therefore, a capacitance of $350 \mathrm{~F} / \mathrm{g}$ and charge densities of $\sim 400 \mathrm{C} / \mathrm{g}$ (considering that the maximum voltage that can be obtained from an aqueous solution is of the order of $1.2 \mathrm{~V}$ ) are possible in principle. While organic electrolytes and ionic liquids may have a larger voltage limit, a correspondingly reduced $\varepsilon_{r} \square($ e.g., $\sim 40$ for acetonitrile) and a higher ionic diameter may imply that the $C_{d l}$ would be reduced and the net charge capacity may not be significantly altered.

\section{electrochemical capacitors: Ionic Liquids}

Ionic solids/salts with low melting points, $T_{m p}$ (say $\left.<100^{\circ} \mathrm{C}[84]\right)$ are termed as ionic liquids. The low melting point is a consequence of bulky organic cations coupled with relatively smaller anions - the effect of size with respect to the melting point is well illustrated through a comparison of the ionic solid, $\mathrm{NaCl}$ (with a $T_{m p}$ of $803{ }^{\circ} \mathrm{C}$ ), while an ionic liquid BMIM-TFSI ( butyl-methylimidazolium and bis(trifluoromethylsulfonyl)imide, respectively) has a $T_{m p}$ of -22 ${ }^{0} \mathrm{C}$. As there is considerable flexibility in cation-anion combinations, there exist enormous possibilities for the synthesis of a variety of ionic liquids.

The relevant attributes of ionic liquids (ILs), that makes them useful as electrolytes in charge transfer applications, e.g., involving batteries[85] or ECs[86], is their (i) relatively high 
ionic conductivity - due to the high ionic density as well as tendency for dissociation, (ii) high electrochemical stability windows (ESW) - due to strong bonding interactions, as well as favorable physical parameters, such as (3) low vapor pressure and viscosity (which is found to be inversely proportional to the conductivity). The use of ILs, in the form of solvent free electrolytes, has then been considered for $\mathrm{Li}$ ion batteries due to the much lower flammability compared to organic electrolytes. The ability to tailor IL characteristics also makes it feasible to design compounds with minimal reactivity towards Li ions, reducing side reactions. However, IL cationic intercalation within the graphitic anode has been seen to reduce the stability of the electrolyte as well as the SEI layer (see Section 4.5) formed on the anode. Interestingly, ILs with longer chain lengths, where van der Waals and hydrogen bonding interactions are also important, were shown to exhibit better stability. Consequently hybrid electrolytes constituted from an IL (about 30-40\%) - organic electrolyte mixture have been advocated for stable SEI film formation while reducing the flammability.

For ECs, in addition to the favorable electrical and physical characteristics, the electrochemical attributes related to the large voltages (which could be as high as $6 \mathrm{~V}[87,88]$ ) over which the ILs are stable), and which corresponds directly to a large energy and power densities, (see Section 2.3). This is especially pertinent in that organic electrolytes are generally unstable beyond $\sim 3 \mathrm{~V}$. However, as the relatively large viscosities in ILs contribute to a greater equivalent series resistance (ESR)- see Section 3.3.1, limiting the power density, efforts are underway to reduce the viscosity while maintaining the favorable characteristics, e.g., the use of IL-organic hybrid electrolyte systems has been advocated for lowering the ESR. A possible lowered capacitance (which negatively impacts the energy density) was reported [89]in porous activated carbon based systems using ILs as electrolytes, presumably due to the larger ion sizes 
and suboptimal wetting (due to the greater polarizability of the ILs) characteristics (see Section 4.8.4). Pore volume occupied by the bulky ILs may also be a factor in terms of reducing the volumetric energy/power densities.

\section{electrochemical devices}

At the very outset, it would be pertinent to understand the performance of present day batteries and electrochemical capacitors in the larger context of the maximal theoretical capacity. This would be important in understanding the factors limiting the achievement of the maximal energy and power density as well as to explore what can be done to bridge the high energy and power densities relevant to the respective technologies. The capacity is considered for the electrodes, i.e., anodes and cathodes, individually as they serve as a source or sink for the electrons (see Section 2.2).

\section{progress in enhancing charge \& energy storage}

The intrinsic charges in a material may broadly be classified as either fixed or mobile encompassing both electrons and ions (cations and/or anions). The electrical capacity/capacitance arises from both the entities and is directly proportional to the charge. Typical methodologies aimed at calculating the maximum charge capacities (in milli-Amperehour $(\mathrm{mAh}) / \mathrm{g}$ ) that could be theoretically achieved involves dividing 96,487 $\mathrm{C}$ (= 1 Faraday of 
charge) /molar weight (in grams), e.g., with carbon with an atomic weight of $12 \mathrm{~g}$, this would correspond to $8040 \mathrm{C} / \mathrm{g}$, or equivalently $2233.5 \mathrm{mAh} / \mathrm{g}$ (for batteries the charge density is almost universally considered in terms of $\mathrm{Ah} / \mathrm{g}$, with $3.6 \mathrm{C}=1 \mathrm{mAh}$ ). It is also then intuitive that the lowest molar weight solid element (which would be Lithium: $\mathrm{Li}_{3}$ ) would provide a high theoretical charge capacity. In terms of obtaining even higher energy density, it would be pertinent to consider the modalities of uptake of hydrogen as well. Initial reports predicted storage capacities, ascribed mostly to physisorption, of the order of $10 \mathrm{wt} \%$ hydrogen[90] and much higher than activated carbon into nanostructures, such as single walled CNTs. However, later studies[91] indicated orders of magnitude lower capacity at $\sim 0.1 \mathrm{wt} \%$. While the initial experiments probed hydrogen adsorption through temperature programmed desorption spectroscopy, the latter studied electrochemically the release of both the physisorbed and chemisorbed hydrogen through cyclic voltammetry (see Section 3.1). Consequently, Li seems to be the element of choice for charge storage at the present.

However, elemental Li by itself is very reactive and hence susceptible to self-discharge (whereby $\mathrm{Li}^{+}$converts to $\mathrm{Li}$ ) and would be best utilized in a compound form[92], where the $\mathrm{Li}$ can be reversibly extracted/inserted into the compound structure, as considered through intercalation schemes (Section 2.2.1). Recourse to binary phase diagrams can be used to establish whether stoichiometric line compounds or solid solutions form and the former are generally preferred. For example, when lithiated graphite anodes are used, yielding a $\mathrm{LiC}_{6}$ stoichiometry, the theoretical capacity is reduced to $\sim 372 \mathrm{mAh} / \mathrm{g}(=2233.5 / 6)$, as six carbons interact for every Li ion that is involved in the charge transfer (from the governing oxidation reaction, Eqn. (6), i.e., $\mathrm{LiC}_{6} \rightleftharpoons \mathrm{Li}^{+}+6 \mathrm{C}+e^{-}$). For $\mathrm{LiC}_{6}$ (with a molar weight of $79 \mathrm{~g}$ ), the electronic charge density would be that corresponding to 1 mole of electrons and would be given as $1221 \mathrm{C} / \mathrm{g}$ (=96, 
487/79) or equivalently $340 \mathrm{mAh} / \mathrm{g}$ ). In present usage, for a typical Li ion battery of 520 $\mathrm{mAh} / \mathrm{g}$ (from the sum of the graphite anode capacity of $\sim 370 \mathrm{mAh} / \mathrm{g}$ and typical oxide cathode capacity of $\sim 150 \mathrm{mAh} / \mathrm{g}$ ) this would imply a $Q_{\text {total }}$ of $\sim 1872 \mathrm{C} / \mathrm{g}$. This would correspond on the average to a capacitance, $C$ of $1070 \mathrm{~F} / \mathrm{g}$ (assuming $\left.Q_{\text {total }}=C V_{O C} / 2\right)$ with a $V_{O C}$ of $3.5 \mathrm{~V}$. In terms of energy density, i.e., $C\left(V_{O C}\right)^{2} / 2$, a value of $6553.8 \mathrm{~J} / \mathrm{g}$ may be estimated. The energy density has also been reported in terms of $\mathrm{Wh} / \mathrm{kg}$ (where $1 \mathrm{Wh}=3600 \mathrm{~J}$ ), which for $6553.8 \mathrm{~J} / \mathrm{g}$, results in $1820 \mathrm{Wh} / \mathrm{kg}$. However, as will be seen in this section and in the next, practical energy capacities are an order of magnitude lower. The considered energy density values for batteries clearly indicates the difference in terms of energy density with an electrostatic/electrochemical capacitor, where the capacitance was considered to have an upper limit of $350 \mathrm{~F} / \mathrm{g}$ (see the end of Section 2.3.2).

Generally, aspects related to (a) the structure (layered carbon layers in graphite can more easily accommodate the Li ions), (b) matching of the chemical potentials of the anode with the energy levels of the electrolyte (see Figure 1), as well as (c) economic factors, dictate practical electrode choice. Similar considerations have been applied for the cathode, the cost of which is a major issue (presently estimated to be $\sim 4$ times the cost of the anode[36]), as well. The vast effort and development in Li ion batteries has led to the use of various Li oxide based compounds depending on the application, e.g., (1) $\mathrm{LiCoO}_{2}$ based systems (such as $\mathrm{Li}$ $\left.(\mathrm{Ni}, \mathrm{Mn}, \mathrm{Co})_{1 / 3} \mathrm{O}_{2}\right)$ for high energy density $(\sim 200 \mathrm{Wh} / \mathrm{kg})$, (2) $\mathrm{LiFePO}_{4}$ based systems for high power density ( $4 \mathrm{~kW} / \mathrm{kg}$ ) and long cycle-life (up to 10,000 cycles). It is pertinent to realize that the record energy density to date (2014), for the Li ion batteries seems to be $\sim 200 \mathrm{Wh} / \mathrm{kg}$, with an approximate annual growth rate of $\sim 6 \mathrm{Wh} / \mathrm{kg}$, which seems to be almost an order of magnitude smaller compared to what may be achievable. While such a rate of progress seems to 
be adequate to reach the US Department of Energy (DOE) target of $250 \mathrm{Wh} / \mathrm{kg}$ in a few years, alternate goals suggest that energy densities of the order of $500 \mathrm{Wh} / \mathrm{kg}$ may be needed for largescale electric vehicle (EV) deployment. This would indicate a necessary energy of $\sim 50 \mathrm{kWh}$ if $100 \mathrm{~kg}$ of battery weight was used.

In terms of future progress, it might be obvious that a very large $\mathrm{Li}$ : $\mathrm{X}$ ratio, where $\mathrm{X}$ is the added element, would be desirable, e.g., looking at phase diagrams we see that the Li:Sn, Li:Si or Li:Ge ratios in the plausible line compounds could be as large as 4.4. Indeed, the theoretical capacity of a $\mathrm{Li}_{4.4} \mathrm{Si}$ compound has been considered[93] to be $\sim 4212 \mathrm{mAh} / \mathrm{g}$ - even larger than that for metallic Li (the capacity for which can be estimated to be $\sim 3860 \mathrm{mAh} / \mathrm{g}$, assuming an atomic weight of $6.94 \mathrm{~g})$. The ratio could be even higher in $\mathrm{Li}: \mathrm{Pt}(5: 1), \mathrm{Li}: \mathrm{Hg}(6: 1)$ and $\mathrm{Li}: \mathrm{Ag}$ (9:1). However, the repeated removal and insertion of Li into such compounds as well as a greater variety of possible compounds, can cause a large relative volume change (defined through the ratio of the change of volume between the lithiated and de-lithiated phases to the volume of the lithiated phase[94], as large as 400\%) and leads to a loss of structural integrity. Generally, while a single-phase is preferred, examples of facile Li intercalation in compounds undergoing phase transitions have been observed as in the cases of $\mathrm{LiCoO}_{2}$ and $\mathrm{Li}_{4} \mathrm{Ti}_{5} \mathrm{O}_{12}$, due to the structural similarity of the transforming phases[8]. Consequently, similarity of the crystal structure of the formed phases may be a relevant criterion for reversibility and cycling.

While silicon nanowires spaced at a distance to accommodate the volume change have been proposed[95], the wires are nevertheless placed on a substrate and isotropic volume change may lead to uneven and poor contact with the substrate negatively impacting the contact resistances. Soft matrices (incorporating polymeric hosts) or hierarchical architectures, which 
accommodate the expansion, have been proposed using, e.g., "yolk-shell" [96] or "pomegranate"- like designs[97] - where the Si nanoparticles which take up the Li are individually placed in spheroidal spaces in a carbon based conductive framework. Consequently, future battery devices may have to rely on a hybrid approach, where a mixture of $\mathrm{Li}, \mathrm{C}$, and other elements such as Si (in an amorphous form) are used, involving more complex designs and greater expense. It would also be intriguing to explore whether the strain accommodation, which may lead to domain formation (e.g., cubic and tetragonal domains in $\mathrm{Li}_{x} \mathrm{Mn}_{2} \mathrm{O}_{4} ; 0<x<1$ ) leads to charged domain boundaries as has often been observed with grain boundaries[98]. The observation of higher $\mathrm{Mn}$ oxidation states, observed in the preparation of $\mathrm{Li}-\mathrm{Mn}-\mathrm{O}$ electrodes through nanograin induced ball-milling methods may also be related to boundary-related effects[99]. It has also been considered that the formation of a space charge zone near the grain boundaries can enhance matrix conductivity. Charges at/near the boundaries have been shown to modulate the electronic conductivity, e.g., an excess positive charge has been observed on the grain boundaries of ionic solids such as $\mathrm{SrTiO}_{3}$ leading to a reduced hole carrier and oxygen vacancy concentrations and therefore an increased electronic and ionic conductivity[100] and related electro-thermal characteristics[101]. The application of boundary/defect pertinent influences in the context of battery charge storage, e.g., fixed $v s$. mobile charge, would open new frontiers with regard to understanding the limits of charge storage as well as correlating the degree of reversibility to phase and domain formation.

In terms of commercial deployment, say for high charge capacity anodes has been the use of metallic elements such as $\mathrm{Al}$ or Sn metals/alloys with a large propensity for interaction with Li. Alloying and conversion reaction based Li ion batteries (see the beginning of Section 2.2.1) are intrinsically susceptible to polarization as well as capacity losses and may require the 
use of catalysts or stabilizing/amorphous coatings [45]. It was reported, e.g., that amorphous SnCo systems with disordered structures (indicated through broad x-ray diffraction peak widths) seemed to have a large cycle life. The culmination of such studies was the commercial introduction by Sony, Japan, in the year 2005, of a $\mathrm{Sn} / \mathrm{Co} / \mathrm{C}$ based amorphous/nanocrystalline anode based composite battery (termed the Nexelion ${ }^{\circledR}$ 14430: $14 \mathrm{~mm}$ in diameter and $43 \mathrm{~mm}$ tall). A Li (Ni, Mn, Co) $\mathrm{O}_{2}$ cathode was used, with the $\mathrm{Ni}$ : Mn ratio of $\sim 1.7$. A capacity increase compared to conventional anodes, was reported yielding a relative $10 \%$ increase in energy density of $\sim 160 \mathrm{Wh} / \mathrm{kg}$ and $20 \%$ increase in volumetric energy density of $\sim 480 \mathrm{Wh} / 1$ [102]. The single-phase behavior of an amorphous Sn-Co alloy yielding a flat voltage discharge profile with greater cyclability compared to crystalline Sn (with a reported gravimetric density $\sim 1000$ $\mathrm{mAh} / \mathrm{g}[103])$ was indicated[104]. It was surmised that while the $\mathrm{Sn}$ is the electroactive element, the Co and $\mathrm{C}$ present in the matrix help in better Li cycling. Typical to the nanoscale (see Section 1.1) the attachment of the active elements to the current collector may pose problems in achieving low electrical resistances and limiting the power capability of such devices. While "nano-architectured" electrodes, e.g., comprising $\mathrm{Cu}$ nanorods plated with active $\mathrm{Fe}_{3} \mathrm{O}_{4}$, have been employed [46] to facilitate such attachment and reduce the resistance, the activation energy barrier for the conversion reaction (see the beginning of Section 2.2.1) seemed to be unaffected.

While the large charge capacities may be preserved through such methods, the power capability ( $C$ rate) could be severely compromised. It has also been recently thought [105] that while a range of compounds could be used for Li conversion and charge storage, only fluorides seem to be suitable for reversible cathode materials. While considerable work needs to be done to understand the basic charge storage mechanisms vis-à-vis reversibility criteria in such systems, preliminary studies have indicated the necessity for a three-phase conversion process of 
the kind: $\mathrm{MF}_{2}+2 \mathrm{Li}^{+}+2 e^{-} \rightarrow 2 \mathrm{LiF}+\mathrm{M}(\mathrm{M}=\mathrm{Fe}, \mathrm{Cu}$, etc. $)$ with no intercalation involved. Such a conversion reaction, say, on the first charge cycle, facilitates the breakup of the agglomerated $\mathrm{MF}_{2}$ particles and promotes further ionic and electronic diffusion. Consequently, a relatively high overpotential (of $\sim 0.8 \mathrm{~V}$ ) would be needed for the initial lithiation, which subsequently decreases to $\sim 0.3 \mathrm{~V}$ as the $\mathrm{MF}_{2}$ particle size is reduced to $\sim 5 \mathrm{~nm}$. As LiF was an ionic insulator, the $\mathrm{Li}^{+}$diffusion was indicated (based on extensive high resolution TEM as well as electron energy loss spectroscopy: EELS) to proceed along the LiF-Fe nanocomposite interface while the electronic conduction occurred along a percolating Fe network. An optimal diffusivity of the M ion also seemed to be necessary (e.g., a higher diffusivity for the $\mathrm{Cu}^{2+}$ compared to $\mathrm{Fe}^{2+}$ yielded a greater degree of reversibility for a $\mathrm{FeF}_{2}-\mathrm{C}$ system), indicating that subtle differences in the morphology at the nanoscale (e.g., it was found that $\mathrm{Cu}$ particles were larger) as well as atomistic considerations may need to be understood.

From a fundamental point of view, the charge capacity could be further enhanced only through (a) increasing the total amount of charge that could be harnessed in a given redox couple, as well as through (b) enhancing the voltage range of the electrodes along with the stability of the electrolytes over such a voltage range. For (a) we have previously discussed one such method, where multiple redox couples could be harnessed through the contributions of both the electrons and the holes (see the discussion in Section 2.2.2, as related to Figure 6). However, a major issue is that the structure should be large enough to accommodate the multiple intercalating ions without adversely affecting the structural stability [95], which has been found to be difficult[40]. Associated and alternative techniques to enhance the charge capacity hinges on the use of elements rather than compounds, since delocalization of charge is comparatively larger in the former enabling rapid charging and discharging. For increasing charge density it 
would then again be necessary to have a large number of electrons transferred per a given redox reaction (with reference to Eqn. 2) combined with a low molar weight. Such considerations are important in achieving the maximum possible charge storage.

2.4.2

Reaching the limits of

charge storage

If the aim is to achieve a 300 mile driving range - common to existing gasoline powered passenger automobiles, the goal of energy density would be in the range of $\sim 700 \mathrm{Wh} / \mathrm{kg}$. The necessary tripling of the energy density, from state-of-the art values, has then been a major motivator for continued research and development in both intercalation based $\mathrm{Li}$ ion batteries as well as new chemistries. Another strong imperative from the scientific side has been that the obtained energy density values seem to be much smaller than the theoretical maxima, with possibly an order of magnitude feasible. We will first discuss the absolute limits and then probe current efforts aimed at reaching such limits.

As the largest concentration of electrical carriers are in a metal, it would be interesting to consider the capacity of a good metal, say copper, with an electrical carrier i of $10^{23} / \mathrm{cm}^{3}$. From definition, the energy capacity would be proportional to the product of the total charge, $Q_{\text {total }}$ transferred multiplied by the $V_{O C}$. The total charge would correspond to $1.6 \cdot 10^{4} \mathrm{C} / \mathrm{cm}^{3}$ (= $1.6 \cdot 10^{-19} \mathrm{CX} 10^{23} / \mathrm{cm}^{3}$ ), or $\sim 1800 \mathrm{C} / \mathrm{g}$ (with a bulk copper density, $\rho$ of $8.9 \mathrm{~g} / \mathrm{cm}^{3}$ ). Such a charge would correspond to an equivalent capacitance (assuming a voltage of $3.5 \mathrm{~V}$ ) of the order of $\sim 514 \mathrm{~F} / \mathrm{g}$ and energy densities $\left(=C V^{2} / 2\right)$ of the order of $3150 \mathrm{~J} / \mathrm{g}$. Converting to commonly used factors, the equivalent maximal values are in terms of the charge capacity is $\sim 500 \mathrm{mAh} / \mathrm{g}$ 
and in terms of the energy density, of the order of $875 \mathrm{Wh} / \mathrm{kg}$. Alternatively, if Li with a reduced density $\left(\rho \sim 0.5 \mathrm{~g} / \mathrm{cm}^{3}\right)$ assuming a similar charge density (of $10^{23} / \mathrm{cm}^{3}$,) is used, there would be a further increase by a factor of $17.8(=8.9 / 0.5)$ implying an energy density of $\sim 15,575 \mathrm{Wh} / \mathrm{kg}$ consequent to an increase in the charge storage capacity to $8900 \mathrm{mAh} / \mathrm{g}$. Considering that many state-of-the art batteries/fuel cells have energy and charge capacities orders of magnitude lower, it may be surmised that the above estimates are upper limits to the amount of charge/energy capacity that may be achieved.

However, it has been estimated recently[106] that a $\mathrm{Li} / \mathrm{F}_{2}$ electrochemical system (corresponding to the most electropositive and electronegative elements in the periodic table) has an estimated theoretical energy density of $\sim 6294 \mathrm{Wh} / \mathrm{kg}$. The thermodynamically allowed energy densities of many battery systems have been tabulated[106] from dividing a known $\Delta G$ (= - $z F \Delta V$; See Section 1.1) by the molar weights of the species involved. While values of $~$ $11,586 \mathrm{Wh} / \mathrm{kg}$ have been quoted for $\mathrm{Li} / \mathrm{O}_{2}$ batteries [42], proper normalization more than halves the value to $\sim 5,217 \mathrm{Wh} / \mathrm{kg}[106]$. The enhanced energy values seem to be more of a function of the reduced molar mass (say, compared to $\mathrm{LiCoO}_{2}$ ) as the $V_{o c}$ values are relatively modest (i.e., $2.9 \mathrm{~V}$ and $2.3 \mathrm{~V}$ for $\mathrm{Li} / \mathrm{O}_{2}$ and $\mathrm{Li} / \mathrm{S}$ systems, respectively). Even considering that the typical real energy density is a factor of two/three below the theoretically estimated maximal density (due to taking the total weight of the electrolyte, see also Section 5.2) there seems to be scope for further improvement. Recent reports on the performance of $\mathrm{Li} / \mathrm{S}$ batteries, for example, seem to suggest energy capacities approaching $1000 \mathrm{Wh} / \mathrm{kg}$, on first charge which unfortunately decreases threefold after just 50 cycles[107]. 
Additionally, there may be corrosion and packaging related issues involved in reaching the posited charge and energy density limits. For example, in the $\mathrm{Li} / \mathrm{O}_{2}$ system (typically involving a $\mathrm{Li}$ metal anode with the production of $\mathrm{Li}^{+}$ions, which migrate to a porous carbon cathode and are reduced through coupling with $\mathrm{O}_{2}$ ) using non-aqueous electrolytes, there is a necessity for excess $\mathrm{Li}$ in the anode. For the cathode, the coupled in $\mathrm{O}_{2}$ needs to be contaminant free (e.g., of atmospheric $\mathrm{H}_{2} \mathrm{O}$ and $\mathrm{CO}_{2}$ ) to avoid the formation of undesirable reaction based products. Aqueous systems suffer from deleterious reactions of water with the electrodes, e.g., either reacting with the $\mathrm{Li}$ anode or forming products such as $\mathrm{LiOH}$, which may clog the porous cathode. While protective polymeric membranes (e.g., PTFE: poly- tetrafluoroethane) on the anode and cathode (to prevent ingress of $\mathrm{H}_{2} \mathrm{O}$ and $\mathrm{CO}_{2}$ while allowing $\mathrm{O}_{2}$ diffusion) have been used[108], stringent operating requirements yielded an energy density $\sim 362 \mathrm{Wh} / \mathrm{kg}-$ far below the theoretical estimates. It was suggested that with a $12.7 \mu \mathrm{m}(0.5$ mil $)$ membrane that the water contamination should be less than $3 \mathrm{mg} / \mathrm{cm}^{2} /$ day for the $\mathrm{Li} / \mathrm{O}_{2}$ battery to be operated in the ambient for 5 days with less than $20 \% \mathrm{Li}$ loss. However, given the absence of an ideal $\mathrm{Li} /$ chalcogenide battery, which is further compounded by lack of knowledge of the basic chemistry, progress in this area seems quite difficult given the myriad reactions that could occur in a oxygen reactive system. Essentially, the mechanisms underlying the possible high capacity also yield sufficiently complex chemical reactions that would be difficult to comprehend in a practical context. It would also be important to have a proper comprehension of the metrics that have been used in reporting the energy density considering that laboratory scale devices may not be truly representative of their widespread practical usage, e.g., considering the elaborate issues involved in packaging and customization. 


\section{The proper reporting of energy density}

The monitoring of charge in a structure is done through the measurement of the capacitance (see details in Section 3) as it is charged and discharged under an applied voltage. As a typical practical device is constituted of many parts, i.e., the external contacts, the electrodes, the electrolyte and the pertinent packaging, the charge/discharge characteristics must be inclusive of the parts. As the partition between the various constituents are not completely clear, the numbers for charge or energy density, from the point of a device user, may invariably need to include the weight or volume of the device package. Such definition must be contrasted with widespread usage of extrapolations/conversion factors between the determined characteristics of the electrode weight/volume to the cell characteristics[109]. The use of volumetric energy density as opposed to mass energy density for characterizing nanostructure based devices has also been proposed[110]. The rationale is the presence of abundant equivalent porosity and low packing density (e.g., typically $<1 \mathrm{~cm}^{3} / \mathrm{g}$, for activated carbon[111]), in the nanostructure arrangement, which may lead to overestimates of the energy density. The consequence is a conversion factor/ratio between the mass based energy density and the volumetric energy density of less than 1, e.g., considering the placement of $10 \mathrm{~nm}$ diameter multiwalled CNTs (with intrinsic density[112] of $\sim 2.1 \mathrm{~g} / \mathrm{cm}^{3}$ ) and $0.1 \mu \mathrm{m}$ in height with a spacing of $\sim 20 \mathrm{~nm}$ on a $1 \mathrm{~cm}$ X $1 \mathrm{~cm}$ substrates results in a packing density of $\sim 0.7 \mathrm{~g} / \mathrm{cm}^{3}-$ then an energy density of 10 $\mathrm{Wh} / \mathrm{kg}$ would translate to a volumetric energy density of $7 \mathrm{Wh} /$ liter. Smaller CNT spacings would be needed, say, $<15 \mathrm{~nm}$ as related to the above example to achieve a conversion factor of

1. A convenient comparison for such values are those reported for the Nexelion ${ }^{\circledR}$ battery, of $160 \mathrm{Wh} / \mathrm{kg}$ and $480 \mathrm{Wh} / \mathrm{l}[102]$, where the conversion factor seems to be $\sim 3$. 
Careful attention must also be paid to the EC energy density metrics, especially in the case of nanostructured devices. For example, theoretical estimates of the areal density, e.g., 1500-3000 $\mathrm{m}^{2} / \mathrm{g}$ for CNTs [83] or graphene have been used to indicate that extremely high capacitance/unit mass values may result, through converting from $\mathrm{F} / \mathrm{m}^{2}$ to $\mathrm{F} / \mathrm{g}$ (see end of Section 2.3.2). In the case of graphene, assuming a thickness/spacing/pore width $\left(t_{w}\right)$ of 0.335 $\mathrm{nm}$, the area density, $S$ (in $\mathrm{m}^{2} / \mathrm{g}$ ) $=2 / \rho t_{w} \sim 2714 \mathrm{~m}^{2} / \mathrm{g}$ where $\rho \square$ is the density (assumed to be that of bulk graphite of $\sim 2.2 \mathrm{~g} / \mathrm{cm}^{3}$ ). This value assumes that both sides of the surface are amenable to adsorption (i.e., when only one side is considered, the $S$ is halved to be at $\sim 1357$ $\mathrm{m}^{2} / \mathrm{g}$ ). Higher values of $S$, say $3100 \mathrm{~m}^{2} / \mathrm{g}$ [113] have been reported where the effective density of the packing and not the intrinsic material seems to have been considered. A reduced density of $0.47 \mathrm{~g} / \mathrm{cm}^{3}$ was also reported for graphene synthesized through the microwave exfoliation of graphene oxide[114] which was presumably responsible for the larger $S$. However, caution with respect to the overestimation of the effective area is still valid, as indicated below.

While on the one hand, it has been thought that there would be a saturation in the obtained capacitance values, due to space charge effects, at areal densities of $\sim 1200 \mathrm{~m}^{2} / \mathrm{g}[115]$ corresponding to pore size widths of $\sim 1 \mathrm{~nm}$, it has also been observed that methodologies for estimating areas through gas adsorption kinetics, say through the Brunauer-Emmett-Teller (BET) mechanisms[116] over-estimates the available area, mainly due to the intrinsic assumptions involved in BET based analysis. While the underlying aspect in BET of modeling multilayer adsorption, with negligible interlayer interactions and variation, may be valid for gas adsorption, the extension to a varying electrolyte ion concentration outwards from the electrode is not apparent. For example, the diameters of the ions in aqueous solutions (e.g., $\mathrm{K}^{+}$and $\mathrm{Cl}^{-}$ions at 
$0.3 \mathrm{~nm}$ ) may be comparable to the $\mathrm{N}_{2}$ gas (with molecular diameter $\sim 0.3 \mathrm{~nm}$ ) used in gas adsorption analysis, while the ions with commonly used organic salts such as TEATFB (TetraEthyl Ammonium TetraFluoroBorate) may be much larger, viz., the TEA cation has a diameter of $\sim 0.68 \mathrm{~nm}$, while the $\mathrm{BF}_{4}{ }^{-}$anion is $\sim 0.44 \mathrm{~nm}[117]$. Consequently, it has been argued that all the pores may not be accessible as in many such cases the sizes of the organic salt/solvated ions may be comparable to[75] or bigger than the species used in the gas adsorption studies. The lack of access to the pores may then result in a reported capacitance per unit area larger than the true value (also see Section 4.9.1). However, if wetting of the underlying electrode (see Section 4.8.4) is ensured, and the charge or discharge rates are slow enough to approach thermodynamic equilibrium, then the BET area may indeed be representative of the actual area.

As an alternative to BET based mechanistic analysis for modeling experimentally based gas adsorption data, smaller areal densities have been obtained through density functional theory (DFT) based calculations[76]. Concomitantly, issues related to modeling the pore size distribution for the area estimation may also need to be carefully considered with respect to the validity of the underlying assumptions involving, e.g., slit like pore widths in DFT based calculations, the inertness of the adsorbent, or pore dilation effects[118]. In the case of CNTs, for example, the effect of the cylindrical pore shapes with varying monolayer structures seemed to give a ratio of the BET determined area to the geometric area as high as $\sim 3$, the ratio being larger for smaller diameter CNTs[119].

In light of the discrepancy with respect to the area density, say through BET determination, it is perhaps better to report the capacitance values in units of $\mu \mathrm{F} / \mathrm{cm}^{2}$ (where the area is considered with respect to the projected area and a roughness factor may be introduced - 
see Section 4.8.4) as this is more closely related to experimental measurement. Simply, a capacity of $100 \mathrm{~F} / \mathrm{g}$ would then translate to $\sim 10 \mu \mathrm{F} / \mathrm{cm}^{2}$ with a determined specific surface area of $1000 \mathrm{~m}^{2} / \mathrm{g}$ (as would be typical to activated carbons used for ECs - see Section 4.9). While mass capacitance densities are undoubtedly useful, they are really pertinent for the overall device, which includes components besides the electrodes.

A reasonably comprehensive [120] review of the best practice methods for evaluating EC performance indicates appropriate test fixture protocols as well as measurement procedures, which could be adopted for comparison between different experimental results. While realistic characteristics, say pertaining to the equivalent resistance (ESR)- crucial for determining the power density - can indeed be only obtained through the construction of the cell that would be ultimately used, laboratory protocols that evaluate electrode characteristics could employ more reasonable testing procedures. This includes, e.g., (a) proper usage of three - electrode vs. twoelectrode setups. While the former is characteristically used in commercial voltammetry (see Section 3.1), the latter is more typical of a two-terminal capacitor, (b) choice of electrolyte and care in extrapolating to commercial devices. It is well known, for instance, that a larger capacitance can be obtained through the use of aqueous electrolytes compared to organic electrolytes, (c) the use of EC device discharge curves (where the voltage scan rate at a constant current can be used to determine the capacitance - see Section 3.2) to estimate the capacitance, which should be carried out in the $V_{\max }$ to $V_{\max } / 2$ range- as would correspond to practical implementation, (d) the time scales involved should be sufficiently rapid to avoid mass diffusion effects while capturing the relevant electrode transfer characteristics, (e) it must be noted that extrapolation of nanostructure thickness characteristics to those of practically needed commercial 
electrodes (which are typically $0.1 \mathrm{~mm}-0.2 \mathrm{~mm}$ thick) is not well established and would also lead to inaccurate estimates. Moreover, the specific roles and contributions to charge transfer/capacitance of the cation or the anion needs to be investigated, e.g., through cyclic voltammetry (see Section 3.1) by working on the negative and positive potentials with respect to a standard reference, say the point of zero charge (PZC), as was suggested in Reference [120]. However, the measurement with respect to the PZC is not always feasible, e.g., when Faradaic processes are present, they may dominate the double layer currents in an EC (see Figure 9 and related text in Section 3.1 as well as Section 5.1.3). It may then be feasible to carry out the experiments and report the electrode/device capacity with reference to a point of minimum capacitance (PMC). In the next section, we will review the experimental procedures in vogue for monitoring the currents and voltages in typical EC devices.

\section{measurement}

The details of various electrochemical methods, instrumentation, and measurements have been covered in much detail in well-regarded books, e.g., Bard \& Faulkner[79], Holze[121], Linden[34], Kissinger \& Heinemann[122], among numerous others. Consequently, we will only summarize the principles of a few methods that may be used to measure and monitor the amount of charge storage in a given structure/device from the point of view of an EC. In most cases, since charge transfer occurs at/due to the coupled electrode-electrolyte interface, interrogative methods that incorporate both the electrode and the electrolyte, are essential. This implies that 
vacuum based diagnostics and modeling, as is sometimes necessary for the original understanding, is quite difficult to implement[123].

The thermodynamic state of an electrode, in terms of its propensity for charge transfer, may be most conveniently understood through the application of an electric potential/voltage. The electrical current (AC/DC) - as a conjugate electrochemical variable[123], may monitor the kinetic processes, in terms of the consequent rate of charge transfer. The response of a time dependent current (/voltage) to a steady-state/dynamically impressed voltage (/current) then forms the basis of monitoring charge transfer and derivatives such as the associated impedance (i.e., incorporating resistance, capacitance, and inductance). Auxiliary techniques, e.g., ellipsometry, Raman spectroscopy, in situ/operando scanning microscopy, etc. have also been used to correlate the change in electrode surface characteristics and the hybrid energy levels, but have generally suffered from lack of precision due to the presence of trace impurities (even at the levels of parts per billion) as well as the complex nature of the electrode-electrolyte interface.

Generally, the capacitance, $C$, is a measure of the change of charge $(d Q)$ to a given change in voltage $(d V)$, and the net charge over an impressed voltage range is given by:

$$
\int_{Q_{1}}^{Q_{2}} d Q=\int_{V_{1}}^{V_{2}} C(V) d V
$$

When the $C$ is constant over the entire voltage range being considered (which is not always true - see Section 5.1), the $\Delta Q=C \Delta V$, where $\Delta Q=Q_{2}-Q_{l}$ and $\Delta V=V_{2}-V_{l}$. The important point is then that since there is no absolute potential reference, say, $V_{l}$, only the difference between two charged configurations and not the absolute magnitude of charge can be measured. A convenient voltage reference - for the potential values on the horizontal axis in CV- is the PZC, which can 
be determined through measurements. Values more positive (/negative) compared to the PZC value are favorable for probing anion (/cation) related adsorption processes and associated electrochemical kinetics. The transients at either ends of the CV scan, which occur, when the potential scan is inverted could indicate the relative influences of the ion adsorption and relaxation times. However, the mode of charge accumulation, i.e., whether charge accumulates as mobile or static charge, is not apparent through such thermodynamic measurements. The understanding of such distinctions is important for correlating the mechanisms of charge transfer in an electrochemical reaction where decay in the performance may be due to the interconversion between these two types. We first discuss a model technique where the independent variable -the applied voltage is a function of time $(t)$, i.e., $V(t)$, as used as in cyclic voltammetry $(\mathrm{CV})$ to study diffusional and electron transfer processes involved in charge storage.

The CV technique involves perturbing an electrochemical system away from equilibrium by applying a triangular voltage waveform, between two electrodes (the working electrode and a reference electrode): Figure 9 (a) and measuring the electrical current (I) response: Figure 9 (b). While a reference electrode (e.g., a standard calomel electrode) is present in a three-electrode test cell, this is dispensed with in a two electrode EC. The potential difference in the former is applied between the test/working electrode and the reference electrode, while the measured current is reckoned between the test electrode and a counter 
electrode. The charge involved in a particular electrochemical process may be monitored through, $Q(t)=\int_{I_{1}}^{I_{2}} I(t) d t$, while the area enclosed by the $I-V$ plot is a measure of the electrical power which multiplied by the time yields the expended energy. The rate of change of voltage is referred to as the scan rate $(s)$ and informs on both the thermodynamics and kinetics of charge transfer. The lower and upper limits, i.e., in the range: $0<s<\infty$, correspond to the thermodynamic equilibrium and kinetically limited regimes, respectively. More practically, the range would be much more limited as relevant electro-physical processes from chemical reaction velocity to electron transfer and ion diffusion are of finite speed. As extensive monographs exist on the performance and conduct of CV, e.g., Reference [124], we restrict our attention here to the significant features of a typical CV plot - see Figure 9 (b).

\section{1 .1}

\section{Characteristics of a}

\section{typical CV plot}

The generic peaked shape of the curve, in Figure 9 (b), results from competing diffusion and kinetic processes. To explain, at time $t_{o}$ and an electrical potential/voltage: $E_{o}$ (point A), $O$ is the abundant redox species. When the applied potential is now decreased (the IUPAC convention indicates decreasing voltage as we move from the left to the right with cathodic/negative currents, say $i_{C}$, at the top and with anodic/positive currents, say $i_{a}$, at the bottom[124]), at some potential close to $E_{o}^{\prime}$ the reduction of $O$ to $R$ (see Eqn. 2) will begin to occur. As the potential decreases further past $E_{o}^{\prime}$, the $O$ begins to reduce more rapidly and a diffusion layer forms at the surface of the working electrode. Once the diffusion layer becomes sufficiently thick such that 
the diffusion of $O$ to the working electrode is equal to the diffusion of $R$ away from the electrode, a peak current $i_{p}$ is reached at a potential $E_{p}$. The difference between $E_{p}$. and $E_{o}^{\prime}$ is related to an overpotential and proportional to the overall system resistance. Past $E_{p}$ the diffusion of $R$ away from the working electrode is sufficiently large to impede diffusion of the $O$ to the relevant/working electrode, which then depletes at the electrode surface. As a result of the greater flux of $R$ away from the working electrode (compared to the flux of $O$ to the working electrode), and the increasing diffusion layer thickness $(\delta \sqcup)$ the current begins to decay through being proportional to $z F A I_{o} D / \delta$. The $z$ is the number of transferred electrons for the $O$ to $R$ reaction, $A$ is the electrode surface area, $I_{o}$ is the bulk concentration of the species undergoing the reaction, and $D$ is the relevant $O / R$ species diffusion coefficient. Finally, at the $i_{\text {lim }}$ no Faradaic reactions occur at the electrode surface. Once $E_{\lambda}$ is reached, the reverse reaction begins to occur and $R$ begins to diffuse to the working electrode. Close to $E_{o}^{\prime}$, the $R$ begins to oxidize to $O$ until a peak current is again attained, now with the opposite (i.e., anodic) polarity. Past $E_{p}$ the current decays until the limiting current is reached. A proper setting of the $E_{\lambda}$ is hence necessary to observe all such features. For instance the $E_{\lambda}$ must be sufficiently far away from the $E_{p}$ to avoid convolution with Faradaic currents, since the former represents the potential at which the doublelayer restructures itself due to a change in the electrode polarity. For instance, when the electrode polarity changes from (+) to (-), negatively charged ions in the IHP and OHP (Section 2.3.1) are repelled electrostatically by the (-) charge on the electrode, while positively charged ions are attracted. In the limiting case of $E_{\lambda} \gg E_{p}$, the $i_{d l}$ can be approximated by difference between the baseline current $i_{b}$ and the $i_{\text {lim }}$ as defined by: 


$$
C_{d l}=\frac{i_{d l}}{A \cdot s}=\frac{\left|i_{b}-i_{\lim }\right|}{2 A \cdot s}
$$

As can be seen from Figure 9(b), the anodic and cathodic peak currents are considered with respect to the their respective baseline currents $i_{b}$ - which results from charge migration from the bulk solution to the surface of the electrode, causing ions to stack preferentially near the surface of the electrode based on the electrode polarity through the double layer (Section 2.3.1). The measured $C_{d l}$ would then be dependent on the applied potential through $i_{l i m}$ and the Helmholtz model would not strictly hold. Consequently, a diffusion capacitance $\left(C_{D}\right)$ based model may need to be used (Section 2.3.2). However, if the capacitance is large and the potential range under investigation is relatively small, $C_{d l}$ could be approximated to be independent of voltage over the specific voltage range. The Faradaic capacitance $\left(C_{F}\right)$ associated with the anodic/cathodic peak currents can also be determined. Generally, the $C_{F}$ is not a capacitance in the typical sense, since it results from non-transient currents associated with charge transfer. The term is defined through:

$$
C_{F}=\frac{\left|i_{p}\right|}{s}
$$

The $i_{p}$ is graphically determined in CV plots as the difference between the measured current and the $i_{b}$ at the peak potential as indicated in Figure 9(b). A linear fit of $i_{b}$ represents the limiting case of no change in the diffusion layer current near the peak potential and is comprised of $i_{d l}$ and $i_{\text {lim }}$, the accurate estimation of which is crucial. It must be ensured that the $O \leftrightarrow R$ conversion is complete for obtaining the true and relevant capacitance values. Further details on the curve fitting and quantity estimation have been previously elucidated[125].

Generally, the shape of the $C V$ plot (with reference to Figure 9 (b), ) may either be 
constituted mainly from (i) rectangular - indicative of a purely double layer contribution: see Figure 10, also see Figure 46(ii) a Faradaic peak - indicative of a specific redox contribution, as in Figure 9 (b) or , Figure 56 (B), (iii) a sloping ellipsoid, see for example, Figure 56 (A)- which occurs due to a parasitic resistive background or for kinetic reasons [127], where the cycling time is shorter than the ions' relaxation time, or even (d) sigmoidal - when nanoscale electrodes may be involved. While peaks in CV correspond to specific redox/Faradaic reactions, the possibility of multiple states of adsorption of metals/radical species due to a distribution in the binding configurations and energies is well recognized. Such a distribution is manifested in CV through multiple peaks over a voltage range (e.g., of $\sim 0.2 \mathrm{~V}$ in the case of $\mathrm{H}$ adsorption on $\mathrm{Pt}$ surfaces)[123] - also see Section 5.1 and text as related to Figure 46 and Figure 48. The CV plot of a typical battery-like material is indicated in Figure 52.

$\mathrm{CV}$ has also been used to understand the mechanisms involved in the electrode-reactant coupling[128], as to whether the coupling is weak (a non-adiabatic limit) or strong (the adiabatic limit). It was noted that while two alternate methodologies, i.e., (i) based on the calculation of the potential energy curves (and use of the Marcus transition state theory, as discussed later with reference to Figure 23, and a (ii) density of states (DOS) based model - based on a Gerischer diagram based approach (see Figure 30), where the transition probability for an electrode to/from a reactant is calculated, give identical values for the rate constant in the non-adiabatic limit, the results differ for the case of strong coupling/adiabatic limit[129]. Notably, a splitting of the voltammetric peak was predicted by the DOS based methodology for large coupling strength as well as the reorganization energy, and may be evident for adsorbed redox systems/those exhibiting pseudocapacitive behavior (Section 5.1). In 
contrast, with a potential energy based understanding no such peak splitting was evident and the consequence of large coupling strength was only that the peak current would be diminished with a concomitant broadening of the peak width

\section{1 .2}

Reversibility,

\section{irreversibility, and quasi- reversibility}

The peak current ratio $i_{p, c} / i_{p . a}$ as well as the potential peak separation $\Delta E_{p}\left(=E_{p, c}-E_{p . a}\right)$ in the voltammogram can be used to determine the degree of reversibility of a particular oxidation-reduction reaction (Eqn. 2). The relevant charge transfer characteristics are typically indicated through determining the solutions to the flux equations, where the experimental time scales are shorter than the times needed to obtain steady state. Furthermore, charge transfer kinetics are such that they follow Nernstian behavior, i.e.,

$$
E=E_{o}^{\prime}+\frac{R T}{z F} \ln \frac{I_{O}(0, t)}{I_{R}(0, t)}
$$

with $E=E_{i}-v t$, with $E_{i}$ being the initial voltage $R$ as the universal gas constant $(=8.31 \mathrm{~J} / \mathrm{mole}$ $\mathrm{K}), I_{o}(0, t)$ and $I_{R}(0, t)$ being the concentration of $O / R$ at the electrode surface $(x=0)$. Assuming semi-infinite diffusion, Fick's law and the boundary conditions for the forward reaction of $O$ to $R$ (with respective diffusion coefficients: $D_{o}$ and $D_{R}$ ) it can be shown $[79,130]$ that the current, $I$, at any point in the $\mathrm{CV}$ curve is defined by:

$$
i=z F A I_{o} \sqrt{\pi D_{o}} \chi(\varsigma t), \text { with } \varsigma t=\frac{z F}{R T}\left(E-E_{o}^{\prime}-\frac{R T}{n F} \ln \sqrt{\frac{D_{o}}{D_{R}}}\right)
$$

While $\varsigma t$ is a dimensionless parameter, the function $\chi \square \varsigma t$ ) - which has been shown to be 
related to a Fermi-Dirac function[131] must be solved numerically and a maximum current; $i_{p}$ may be obtained:

$$
i_{p}=0.4463 \sqrt{\frac{F^{3}}{R T}} z^{3 / 2} A D_{o}^{1 / 2} I_{o} s^{1 / 2}=2.69 \cdot 10^{5} z^{3 / 2} A D_{o}^{1 / 2} I_{o} s^{1 / 2}(\text { at } 298 \mathrm{~K})
$$

The above relation has been cited as the Randles[132]- Ševčík[133]equation. Assuming Nernstian charge transfer behavior, the potential for which the current reaches a peak value would be:

$$
E_{p}=E_{o}^{\prime}+\frac{R T}{z F}\left(\ln \left(\sqrt{\left(\frac{D_{R}}{D_{o}}\right)^{\gamma}}\right)-1.109 \cdot \gamma\right)
$$

$\gamma= \pm 1$ for the anodic/cathodic sweep. If it is assumed that $D_{O}=D_{R}(=D$, say), then the anodiccathodic peak separation $\left(\Delta E_{p, r}\right)$ is:

$\Delta E_{p, r} \approx \frac{2.3 R T}{z F}=\frac{59}{z} \mathrm{mV}($ at $298 \mathrm{~K})$

Generally, reversible charge/electron transfer considering Eqns. (15) and (16), is therefore defined by three important relationships:

(1) The $i_{p}$ (from Eqn. 15) is proportional to $I_{O}$ or $I_{R}$ as well as $\sqrt{s}$

(2) $i_{p, c} d i_{p . a} \sim 1$,

(3) $\Delta E_{p}$ (from Eqn. 17) is not a function of scan rate or concentration.

For systems exhibiting irreversible charge transfer, e.g., due to adsorption of the redox couple onto the electrode or due to the coupling of homogeneous reactions, which prevent the 
reverse reaction from occurring, the currents diminish with successive voltage sweeps is shown in Figure 10. In this case, only the forward (/backward) reaction needs to be considered, e.g., for a predominant forward reaction, we get:

$$
O+z e^{-} \stackrel{k_{f}}{\longrightarrow} R
$$

Using methods similar to those used for obtaining Eqn. 15, we get for the peak current in the irreversible case $\left(i_{p, i r r}\right)$ and the $E_{p}$

$$
\begin{aligned}
& i_{p, i r r}=2.99 \cdot 10^{5} \alpha^{1 / 2} A D_{o}^{1 / 2} I_{o} s^{1 / 2}(\text { at } 298 \mathrm{~K}) \\
& E_{p}=E-\frac{R T}{n F}\left(0.78+\ln \frac{D_{o}^{1 / 2}}{k^{o}}+\ln \sqrt{\frac{\alpha F v}{R T}}\right)
\end{aligned}
$$

While the $i_{p, i r r}$ is once again proportional to $C_{O}$ and $\sqrt{s}$, the $\Delta E_{p}$ is now dependent on the scan rate as well as the chemical rate constants, unlike in reversible systems (see Eqn. 17). Knowledge of the $\Delta E_{p}$ enables the determination of standard rate constant $k^{o}$ (which is related to the ratio of the forward and the backward reaction rate constants, $k_{f}$ and $k_{b}$, understood through an Arrhenius activation energy based form, through:

$k_{f}=k^{0} \exp \left(-\alpha \frac{F}{R T}\left(E-E_{o}^{\prime}\right)\right), k_{b}=k^{0} \exp \left((1-\alpha) \frac{F}{R T}\left(E-E_{o}^{\prime}\right)\right)$

where $\alpha \square$ is a charge transfer coefficient and a measure of the symmetry of the energy barrier for reduction or oxidation reactions to occur. Typically a value for $\alpha \square=0.5$ is considered a reasonable approximation[79]. The above equations may be rearranged to yield a direct relation between the $\Delta E_{p}$ and the $k^{o}\left(=k_{f} f k_{b}\right)$ may be defined.

The reaction rate constants in turn are fundamental to the Butler-Volmer equations for the 
net current in a redox reaction, where the net current is considered through the difference between the forward $(f)$ and the backward $(b)$ currents, as typical to equilibrium through:

$$
i=F A\left[k_{f} I_{O}(0, t)-k_{b} I_{R}(0, t)\right]
$$

Putting in the forms of Eqn. (18) into Eqn. (19), we may also obtain a more recognized form for the Butler-Volmer equation, as:

$$
i=i_{o}\left\{\exp \left(-\frac{\alpha F}{R T} \Delta E_{p}^{\prime}\right)-\exp \left(-\frac{(1-\alpha) F}{R T} \Delta E_{p}^{\prime}\right)\right\}
$$

The $i_{o}$ is defined as an exchange current (also see Section 4.3 and discussion related to Figure 24) and is formally the cathodic current (or the negative of the anodic current) at equilibrium and $\Delta E_{p}^{\prime}$ is the peak separation voltage in excess of the $\Delta E_{p, r}-$ from Eqn. (17) - akin to an overpotential[80]. An alternative formulation of the Butler-Volmer expression is given later in Section 4.3, as Eqn. (36).

For quasi-reversible systems, which may be considered to be intermediate between reversible and irreversible systems, electron transfer kinetics of the forward (/reverse) reaction are not sufficiently facile and the opposing reverse (/forward) reaction must be taken into account. For a one-step, one-electron transfer the reaction can then be defined through a modification of the basic Butler-Volmer relations (Eqn. 20). The current flux is then given through:

$$
i_{\text {quasi }}=k^{0} \exp ^{-\alpha \frac{F}{R T}\left(E-E_{o}^{\prime}\right)}\left(I_{o}(0, t)-I_{R}(0, t) \exp ^{\frac{F}{R T}\left(E-E_{o}^{\prime}\right)}\right)
$$

The resulting equations for the peak currents $\left(i_{p, q u a s i}\right)$ and the peak separation: $\Delta E_{p, \text { quasi }}$ are given by[79,134]: 


$$
\begin{gathered}
i_{p, \text { quasi }}=F A \sqrt{\frac{D_{o} F S}{R T}} I_{o} \Psi(E) \\
E_{p, q u a s i}=E_{o}^{\prime}+\frac{R T}{F}\left(\frac{1}{z} \ln \left(\sqrt{\left(\frac{D_{R}}{D_{o}}\right)}\right)-\Xi(\Lambda, \alpha)\right) \\
\Lambda=k_{o} \sqrt{\frac{R T}{D F s}}=k_{o} \sqrt{\frac{R T t}{D F\left(E-E_{i}\right)}}
\end{gathered}
$$

It should be noted that the symbols $\Psi(E)$ and $\Xi(\Lambda, \alpha)$ are relatively complex, dimensionless, functions of the potential $(E)$, and the $\Lambda$ (the Matsuda-Ayabe parameter) and are depicted in Figure 11. Consequently, it can be shown that $i_{p \text {, quasi }}$ is not directly proportional to $\sqrt{s}$, due to the additional dependence of the scan rate of the $\Psi(E)$. The peak separation: $\Delta E_{p \text {, quasi }}$ is inversely proportional to the $\sqrt{s}$.

The variation of the cathodic and the anodic peak potentials with the logarithm of the $s$ may also be used to determine the electron rate constant as well as the transfer coefficient ( $\alpha \square \square$ in the Butler-Volmer equation - see Eqn. 20) in diffusionless systems[135]. The basis is from an extrapolation at very large $s \square$ corresponding to complete irreversibility to an $s$ where the equilibrium redox potential is obtained. If the intercepts from the anodic and the cathodic sides meet at the same point, this would correspond to $\alpha=0.5$ [135]: Figure 13. The deviation is a measure of $\alpha$ from which the rate constant $\left(k^{o}\right)$ can be calculated (see Eqn. 19). The initial horizontal part of the curve represents the range of $s$ where close to equilibrium conditions are present. At larger scan rates $\left(s \square>s_{0}\right.$, a critical scan rate), deviations from equilibrium/quasiequilibrium[136] are manifested in a Tafel-like[137] region, where the forward/backward (anodic or cathodic) reactions are preferred. 
The limiting scan rate $\left(s_{\square}\right)$ is implied from the exchange current density, i.e., from $i_{o}=$ $C s_{\square}$. It was proposed that [138] $s_{\square}$ could be considered a surface area independent reversibility parameter, with a relationship between the separation of the irreversible peak $\left(V_{p \text {, irrev }}\right)$ and the reversible peak $\left(V_{p, \text { rev }}\right)$ voltages of the form: $\Delta E_{p}=\left(V_{p, \text { irrev }}-V_{p, \text { rev }}\right)=h\left[\ln (s)-\ln \left(s_{\square}\right)\right]$, where $h=\frac{R T}{z \alpha F}$ and $\alpha \square \square \square \mathrm{s}$ the transfer coefficient for a reaction involving $z$ electron transfer. When irreversibility sets in, the exchange current density $\left(i_{o}\right)$ is very small implying a large charge transfer resistance, $R_{c t}$ :

$$
R_{c t}=\frac{R T}{F i_{o}}
$$

An alternate discussion with respect to a $s / k$ ratio is considered for pseudocapacitive processes (as in Figure 48 in Section 5.1), a critical value beyond which beyond which irreversibility sets in. For adsorbates undergoing redox reactions, this would result in a displacement of the cathodic and anodic peaks in proportion to the increase of $s$ above $s_{\square}$.

\section{Chronopotentiometry}

When a constant input current (applied for a certain time) drives reactions at the electrode surface and the potential of the system is measured, such a measurement scheme is referred to as Chronopotentiometry, and provides complementary information to CV. The principles of this technique are indicated in Figure 14 . When a current $\left(i_{\text {source }}=i_{o}\right)$ is put in: Figure 13 (a), electrons flow into the solution and the $O$ species is reduced at a constant rate at the working electrode surface. Once the surface concentration of $O$ is exhausted, the magnitude of 
the measured potential increases rapidly until another reduction potential is reached, resulting in a step-like function for the measured potential. Since ideally polarizable electrodes do not exhibit redox reactions, the transitions between different redox events should be observed. The voltage response should only exhibit linear behavior as shown in Figure 14 (b).

The time associated with the reaction can be termed a transition time $(\tau)$ and is a function of the diffusion coefficients and surface concentrations $\left(I^{S}\right)$ of the $O$ and $R$, and may be estimated through the Sand equation:

$$
\tau=\left(\frac{z F A I_{O / R}^{S} \sqrt{\pi D_{O / R}}}{2 i_{\text {source }}}\right)^{2}
$$

Analogous to $i_{p}$ and the instantaneous current in $\mathrm{CV}, \tau$ and the measured voltage can be derived analytically by using the semi-infinite linear diffusion boundary conditions and the flux equations. The capacitance can be derived from the slope of the Voltage-time plot, as indicated in the caption to Figure $14(\mathrm{~b})$, where it is seen that the voltage window $(\Delta V)$ and time associated with charging $(\Delta t)$ both play critical roles in influencing the electrode capacitance. For purely double-layer capacitors, $\Delta V$ is determined and characteristically related to the value at which the solvent undergoes electrolysis, i.e., for aqueous solutions $\Delta V$ is $\sim 1 \mathrm{~V}$, while organic solvents, such as acetonitrile, typically have much larger breakdown voltages of $\sim 2.7 \mathrm{~V}[68]$. When a redox couple is added to the solvent-solute system, the current supplied drives Faradaic reactions at the electrode surface until the surface concentration of the redox couple is depleted. The voltage at which this occurs is dependent on the reversibility of the system[125]. Assuming that 
$\Delta V$ is fixed for a given solvent, the Faradaic capacitance: $C_{F}$ can be defined for chronopotentiometric systems as:

$$
C_{F}=\frac{i_{\text {source }} \tau}{\Delta V} \sim \frac{I_{O / R}^{S}}{\Delta V i_{\text {source }}}
$$

with $\tau$ being the time associated with the Faradaic reactions. Furthermore, from Eqn. 26, a maximum $C_{F}$ can be obtained with small source currents and when the surface concentrations are maximal. Additionally, an equivalent series resistance (ESR) may be calculated experimentally through $R_{E S R}=\Delta V / 2 i_{o}$ as indicated in Figure 14 (b). In electrolytic capacitors, $R_{E S R}$ is a combination of the current collector resistance, $R_{s}, R_{c t}$ and the contact resistances of the terminal leads. A low value of $R_{E S R}$ is critical for high power applications, since large resistances can lead to heating and expansion of the electrolyte, causing capacitor failure.

\section{Electrochemical}

\section{Impedance Spectroscopy (EIS)}

An alternate technique that is used to monitor the thermodynamics and kinetics of charge transfer through considering the net impedance in an electrochemical process in the frequency domain, is the EIS[139]. The frequency range may probe and span diffusion processes (in the milli-Hz: $\mathrm{mHz}$ range, corresponding to mass transfer occurring over time scales of seconds to hours) to electron transfer kinetics (in the Mega-Hz: $\mathrm{MHz}$, range, corresponding to much faster time scales related to charge transfer) occurring in electrochemical process. As the EIS technique has been extensively discussed in specialized books $[139,140]$ as well as in 
standard electrochemistry textbooks[79,80,141], we delineate only the salient aspects relevant to nanoscale charge transfer. EIS records such frequency variable response (e.g., through a measured electrical current - which may include contributions from both electrons and ions) of an electrochemical system to an applied stimulus (e.g., through an applied potential difference/voltage).

Generally, a constant DC potential $E_{d c}$ is applied to the system (to set the surface concentrations of the $O$ and $R$ ) in addition to small amplitude AC signal $\left(E_{A C}\right)$ of a given frequency, to induce small perturbations to the equilibrium. The $E_{A C}$ and the resulting current $I_{A C}$ would be out of phase with each other, due to capacitances such as $C_{d l}$ and $C_{F}$, yielding a complex impedance $Z$ (= ratio of $E_{A C}$ to $I_{A C}$ ) - constituted from real (Z') and imaginary (Z’) components, representing the passive (resistive) and reactive (capacitive and/or inductive) contributions, respectively. Two representations are then favored for EIS: (1) The Bode plot, which considers the variation of the amplitude, $|Z|$ and the phase, $\phi\left[=\operatorname{Tan}^{-1}\left(Z^{\prime \prime} / Z^{\prime}\right)\right]$ with frequency, or (2) The Nyquist plot, which plots (by convention [64]) - Z” (on the vertical axis) vs. Z' (on the horizontal axis).

The obtained $Z$ is fitted to integrated resistor-capacitor models with an aim to understand the relevant electrical and electrochemical processes. One popular model is the Randles circuit, indicated in Figure 15. The Warburg impedance: $Z_{w}$ indicated in Figure 15 represents the frequency $(f=\omega / 2 \pi)$ dependent diffusional processes and is constituted from both capacitive and resistive attributes. It can be derived that [79,141]:

$$
Z_{w}=\frac{\sigma}{\sqrt{\omega}}(1-j) ; \text { where } \sigma=\frac{R T}{z^{2} F^{2} A \sqrt{2}}\left(\frac{1}{I_{o} \sqrt{D_{O}}}+\frac{1}{I_{R} \sqrt{D_{R}}}\right)
$$


The net impedance $\left(Z_{n e t, R}\right)$ of the Randles circuit may be obtained through the addition of the resistive and capacitive impedances, with real $\left(Z_{\text {real, } R}\right)$ and imaginary $\left(Z_{\text {imag, },}\right)$ components, i.e.,

$$
\begin{gathered}
Z_{\text {net }}=Z_{\text {real }, R}+Z_{\text {imag }, R} \\
Z_{\text {real }, R}=R_{s} \frac{R_{c t}+\frac{\sigma}{\sqrt{\omega}}}{\left(C_{d l} \sigma \sqrt{\omega}+1\right)^{2}+\omega^{2} C_{d l}^{2}\left(R_{c t}+\frac{\sigma}{\sqrt{\omega}}\right)^{2}} \\
Z_{\text {imag }, R}=\frac{\omega C_{d l}\left(R_{c t}+\frac{\sigma}{\sqrt{\omega}}\right)^{2}+\frac{\sigma}{\sqrt{\omega}}\left(C_{d l} \sigma \sqrt{\omega}+1\right)}{\left(C_{d l} \sigma \sqrt{\omega}+1\right)^{2}+\omega^{2} C_{d l}^{2}\left(R_{c t}+\frac{\sigma}{\sqrt{\omega}}\right)^{2}}
\end{gathered}
$$

The figure represents yet a simplified model in that possible contributions from pseudocapacitance (see Section 5.1) and quantum capacitance (see Section 4.11) have not yet been included. Additionally, the electrolyte capacitances as well as additional parasitic capacitances and resistances have also been ignored. For Faradaic processes with quasireversible/irreversible systems (see Section 3.1.2), a $R_{c t}\left(=R T / F i_{o}\right)$ is defined (where $i_{o}$ is an exchange current - as in Eqn. 24) and is dependent on the relative concentration ratio: $I_{O} / I_{R}$, through the applied DC offset voltage. For instance, when $I_{O}\left(/ I_{R}\right)$ is larger than $I_{R}\left(/ I_{O}\right)$, the diffusion of $R(/ O)$ to the electrode can be hindered. It is also apparent that analytical formulations would quickly get relatively unwieldy with additional capacitances or resistances. However, the high frequency $(\omega \rightarrow \infty)$ and low frequency $(\omega \rightarrow 0)$ examination of Eqns. 28 are often used for insight. For example, in the former limit, the $Z_{w}$ (see Eqn. 27) may be neglected and hence: 


$$
\begin{gathered}
Z_{\text {real }, R}(\omega \rightarrow \infty) \sim R_{s}+\frac{R_{c t}}{1+\left(\omega C_{d l} R_{c t}\right)^{2}} \\
Z_{\text {imag }, R}(\omega \rightarrow \infty) \sim \frac{\omega C_{d l} R_{c t}^{2}}{1+\left(\omega C_{d l} R_{c t}\right)^{2}}
\end{gathered}
$$

The above relations could be combined into a form, that is akin to the equation of a circle in the complex plane, with a center coordinate: $R_{s}+R_{c} / 2$, and radius: $R_{c t}$

$$
\left(Z_{\text {real }, R}-\left(R_{s}+\frac{R_{c t}}{2}\right)\right)^{2}+Z_{\text {imag }, R}^{2}=\left(\frac{R_{c t}}{2}\right)^{2}
$$

Consequently, a plot of the real and measured parts of the complex impedance, as a function of the frequency can be made, as indicated in Figure 16 (a). However, at low frequency $(\omega \rightarrow 0)$ regime, the $Z_{w}$ is dominant as the electrochemical response is diffusion controlled. Consequently,

$$
\begin{aligned}
& Z_{\text {real }, R}(\omega \rightarrow 0) \sim R_{s}+R_{c t}+\frac{\sigma}{\sqrt{\omega}} \\
& Z_{\text {imag }, R}(\omega \rightarrow 0) \sim \frac{\sigma}{\sqrt{\omega}}+2 \sigma^{2} C_{d l}
\end{aligned}
$$

Generally, at sufficiently low frequencies, $Z_{\text {real }, R}$ is dominated by diffusion processes (given by the $\sigma$ in Eqn. 26) and the phase $(\phi)=45^{\circ}$. The transition between the kinetic and diffusion dominated regimes is termed the knee frequency $f_{\text {knee }}$, - see Figure $17(\mathrm{a})$, which also represents a change from resistance dominated to capacitance dominated behavior. The time constant (T.C.) for the charging and discharging cycles is related to $1 / f_{\text {knee }}$. A smaller T.C. is desirable for larger power density devices. If $\phi$ continues to increase from $45^{\circ}$ to $90^{\circ}$ below $f_{\text {knee }}$, 
as with purely double-layer capacitors, the equivalent circuit model shown in Figure 14 is no longer valid. Note that a $90^{\circ}$ phase angle is representative of an ideally polarizable capacitor and the $Z_{w}$ can therefore be replaced with a $C_{F}$. element. A purely capacitive frequency response at low frequencies is indicated in Figure 17(b).

We will first discuss an immediate and important application of EIS, with respect to its usage in characterizing the performance of ECs in terms of representing the device as a distributed resistor-capacitor based equivalent circuit.

An EIS application:

\section{Self-discharge in electrochemical capacitors}

Another irreversible characteristic that plagues capacitive characteristics in particular and energy storage in general is the aspect of self-discharge. EIS based experimentation may be useful to understand the involved processes. The basic idea of self-discharge is that there would be a driving force for the transformation of a high energy charged state to a lower energy discharged state[142] - see Figure 18. As overpotentials are typically required for redox processes (see Eqns. (16) and (23) in Section 3.1.2 and Eqn. (36) in Section 4.3) the selfdischarge process may also be concomitant with the decay of such potentials to the thermodynamic values. The self-discharge process may be modeled in this case through the parallel addition of a charge transfer resistance, $R_{c t}$ from Eqn. (24), to the nominal capacitance. Additionally, any Faradic charge transfer reaction that occurs within the potential window of an EC would yield non-polarizable electrodes with charge transfer across the double layer and/or through a redox shuttle, whereby the ions diffuse between the electrode pairs. Gases, such as 
oxygen or hydrogen, which may be generated in aqueous electrolytes, may also diffuse from an electrode where they are generated to the electrode of the opposite polarity.

The self-discharge for the decay in the overpotential has been typically modeled through the equivalence of the Tafel current (valid for a particular electrode polarization [137]) of the form: $i=i_{o} \exp \left(\frac{\alpha V(t) F}{R T}\right)$ to the loss in the capacitor discharge current: $i=-\frac{d}{d t}[C(t) V(t)]$. It has then been shown [142] that for non-diffusional processes, the $V(t)$ decays as (i) $\ln (t)$ for the case of self-discharge of the overpotential with a decay coefficient proportional to $(R T / \alpha F)$, and as (ii) $\exp (-t / R C)$, for a discharge dominated by Ohmic leakage resistance. Where diffusional processes may be involved, i.e., involving a concentration gradient driven impurity susceptible to Faradaic reactions within the allowed voltage window, a Randles- Ševčík type current, where $I \sim t^{-1 / 2}$ - see Eqn. (15), must be invoked which then implies a $V(t)$ increase as $t^{1 / 2}$ for this case. The mechanisms of self-discharge can then be relatively easily understood through concomitant plots of the voltage decay with time. A consolidated model for the self-discharge in an electrochemical capacitor is indicated in Figure 19. Considering that the self-discharge is an activated process, from Figure 18, an Arrhenius-type relation for the discharge current proportional to $\exp \left(-E_{q} / k_{B} T\right)$ can be written, where the discharge increases with temperature. Typical activation energies $\left(E_{a}\right)$ for Faradaically controlled processes are of the order of $0.4 \mathrm{eV}$ to $0.8 \mathrm{eV}$, while lower for diffusional processes at around $0.2 \mathrm{eV}[142]$. This implies that for $E_{a}=$ $0.4 \mathrm{eV}$, that the discharge current would increase by more than a factor of three for a temperature rise (of $25 \mathrm{~K}$ ) from $298 \mathrm{~K}$ (room temperature) to $323 \mathrm{~K}$. Consequently, there is a large incentive to prevent thermal runaway, which has been considered to be one of the pernicious problems in energy storage management involving capacitors and batteries. Aspects related to self-discharge 
are inevitable whenever Faradic processes are involved, as will be discussed later in Sections 5.1 and 5.2.

3.3.2

\section{Components of the}

\section{impedance}

As discussed earlier, both electrical (i.e., charge transfer) as well as mass transfer oriented processes (e.g., species diffusion) may be modeled through EIS. One may assign a capacitance to each of these processes via the ratio of the magnitude of accompanying charge transfer to the applied potential, as well as a resistance to account for the dissipation concomitant to each process. By way of preliminary introduction, such assignment accounts for terms such as diffusion resistance/capacitance, reaction resistance/chemical capacitance (see Section 4.10), etc. From a broader perspective, thermodynamic considerations related to the fluctuationdissipation theorem[143] are apparent.

Complex Nyquist diagrams, enabling analytical expressions, may be composed from simpler components and principles, e.g., ranging in scale from considering the sum of individual specific resistor or capacitor components to elaborate transmission line models[144,145][146] first used in describing the propagation of electromagnetic waves[147]. The aggregation of all such resistances and capacitances typical to a given representative volume element may be depicted by way of a transmission line model. It is then obvious that the choice of the volume element would be the most difficult in that it should simultaneously (a) be sufficiently simple, with a few representative resistor/capacitor elements, so as to allow insight, while (b) being reasonably comprehensive so as to capture the important and relevant electrochemical processes. 
As an example, for probing charge transfer in an electrochemical device, constituted of two porous electrodes and an electrolyte, the following components each with their own specific resistance and capacitance could be assumed, i.e., (1) macroscopic electrodes separated by an electrolyte, (2) porous electrodes along with the interpenetrating electrolyte, (3) porosity on the pores (akin to micro-or meso-pores on a macroporous system), etc. Such models were developed by de Levie[145,148,149], and a particular equivalent circuit is shown in Figure 20.

Consequently, by utilizing EIS at different DC voltages and frequency ranges, the relative importance of $C_{F}, C_{d l}, R_{c t}$, and $R_{s}$ can be determined. Each of these values is also affected by the electrode, electrode-electrolyte interface, electrode-redox couple interactions, etc. and can thus be used to characterize a range of electrochemical performance. While the amplitude, $|Z|$ and the phase can be experimentally measured over a wide range of frequency for a given practical electrochemical system, the general difficulty is in the interpretation of the data in terms of an equivalent circuit/model, where (a) the values of the resistance/capacitance for individual processes may not be clear (see, e.g., the discussion related to charge transfer in a dye sensitized solar cell - Section 4.10), or (b) many equivalent impedance assignments can be made as the constraining parameters are only the $|Z|$ and the phase. For example, a more comprehensive model - see Figure 21, for the case of nanostructures - may include the additional effects of a redox/Faradaic reaction induced pseudocapacitance (see Section 5.1), space charge capacitance: $C_{s c}$ (see Section 2.3.2) and a quantum capacitance: $C_{Q}$ (see Section 4.11) as well as omnipresent shunt resistances: $R_{s h}$, which arise due to defects and provide leakage paths for the stored charge in the capacitances. 
From a thermodynamic point of view, it has been discussed previously that the charge distribution (and hence the capacitance) at an electrode-electrolyte interface should be a function of the electrical potential (See Section 2.3.2). However, there is often frequency dispersion to the capacitance, i.e., $C(\omega)$, which may need to be considered for realistic modeling of the experimental characteristics. Generally, the frequency dependent complex permittivity, $\varepsilon(\omega)$, is related to the real $\left(\varepsilon_{r}^{\prime}\right)$ and imaginary $\left(\varepsilon_{r}^{\prime \prime}\right)$ components through:

$$
\varepsilon(\omega)=\varepsilon_{0} \varepsilon_{r}(\omega)=\varepsilon_{o}\left[\varepsilon_{r}^{\prime}(\omega)-j \varepsilon_{r}^{\prime \prime}(\omega)\right]
$$

where $\varepsilon_{\mathrm{o}}$ is the permittivity of free space $\left(=8.854 \cdot 10^{-12} \mathrm{C}^{2} / \mathrm{Nm}^{2}\right)$ and $j=\sqrt{-1}$. The electrical impedance, say as $Z(\omega)=Z^{\prime}(\omega)+j Z^{\prime \prime}(\omega)$, may be then calculated from the complex capacitance $C_{S}^{*}(\omega)$ with $\varepsilon_{r}(\omega)=\frac{C(\omega)}{C_{o}}=\frac{1}{j \omega Z(\omega) C_{o}}$ and $C_{o}=\frac{\varepsilon_{o} A}{L}$, with $A$ as the electrode area and $L$ the distance between the electrodes. Consequently,

$$
\begin{aligned}
& Z^{\prime}(\omega)=\frac{\varepsilon_{r}^{\prime \prime}(\omega)}{\omega C_{o}\left(\varepsilon_{r}^{\prime}(\omega)^{2}+\varepsilon_{r}^{\prime \prime}(\omega)^{2}\right)} \\
& Z^{\prime \prime}(\omega)=-\frac{\varepsilon_{r}^{\prime}(\omega)}{\omega C_{o}\left(\varepsilon_{r}^{\prime}(\omega)^{2}+\varepsilon_{r}^{\prime \prime}(\omega)^{2}\right)} .
\end{aligned}
$$

Plots for the frequency dispersion of $\mathcal{\varepsilon}_{r}{ }^{\prime}(\omega)$ and $\varepsilon_{r}{ }^{\prime \prime}(\omega)$ may then be tabulated.

One may then compare electrical circuit models to characterize the $Z(\omega)$ variation in a typical electrochemical system, using, e.g., (i) an equivalent series resistance (ESR) model (see Section 3.3), (ii) a two-dimensional random network of resistors and capacitors (2D RC) model[150,151], and a derivative (iii) constant phase element (CPE) model[140,146,152]. The 
idea behind such various representations was to investigate whether the net impedance, in terms of the resistance and capacitance of an electrochemical system could be considered in terms of lumped or distributed elements, and estimate the minimum number of needed fitting parameters. In the represented ESR model, of Figure 22(a), $R_{E S R \square} \square \omega \square \square$ embodies the frequency dependent dielectric polarization losses and $R_{p}$ accounts for the leakage current in the capacitor. Then, $Z_{\square} \square \omega \square=R_{E S R \square} \square \omega \square \square \square \square j \square \omega C=(\tan \quad(\delta) \square \square j) \quad \square \omega C$, where $\tan (\delta)=-\frac{Z^{\prime}}{Z^{\prime \prime}}=\frac{\varepsilon_{r}^{\prime \prime}}{\varepsilon_{r}^{\prime}} \quad$ is $\quad$ a frequency-dependent loss-tangent defined in terms of the real and imaginary components of the impedance/dielectric permittivity.

Alternately, the 2D RC model of Figure 22(b), may consider the electrode-electrolyte interface in terms of a distributed electrical network of resistors and capacitors. To describe the net electrical conductivity $\sigma_{\text {net }}$ consisting of an admixture of electrolyte (with a conductivity: $\sigma_{\text {elec }}$ ) along with a metallic electrode (with a conductivity: $\sigma_{M}$ ) we may invoke a mixing rule, with the consideration that the measured resistance would be some combination of the electrode and electrolyte resistances in parallel or in series. Assuming equivalent geometries, for the case of parallel addition: $\sigma_{\text {net, }}=p\left(\sigma_{\text {elec }}\right)+m\left(\sigma_{M}\right)$, while for series addition: $\left(\sigma_{\text {net }, P}\right)^{-1}=p\left(\sigma_{\text {elec }}\right)^{-1}+m\left(\sigma_{M}\right)^{-1}$ where $p$ and $m$ are the relative contributions from the electrolyte and the electrode, respectively. Nominally, the sum of $p$ and $m$ would be expected to be equal to unity. We may then write that $\left(\sigma_{\text {net }}\right)^{\vartheta}=p\left(\sigma_{\text {elec }}\right)^{\vartheta}+m\left(\sigma_{M}\right)^{\vartheta}$ (adopting the Lichtnecker mixing rule[153]) with $\square 1 \leq \vartheta \leq 1$, where the upper and lower bounds relate to the parallel and series combinations discussed above. Consequently, for $\vartheta \rightarrow 0$ (assuming an equal number of series and parallel connections), it can be derived that $[154]_{\text {inter }}^{*}=\left(\sigma_{\text {elec }}^{*}\right)^{p}\left(\sigma_{M}^{*}\right)^{m}$. From considering the 
electrolyte with electrical conductivity of: $\sigma_{\text {elec }}\left(=j \omega \varepsilon_{0} \varepsilon_{r}{ }^{\prime}\right)$ and assuming that $\sigma_{M}$ for the metallic electrode is purely real, the complex conductance of such a network can be derived to be $\sigma_{2 D R C}=\frac{(j \omega C)^{\Theta}}{R^{1-\Theta}} Z(\omega)=\frac{L R^{1-\Theta}}{A(j \omega C)^{\Theta}}=\frac{L R}{A(\omega R C)^{\Theta}}\left[\cos \left(\frac{\Theta \pi}{2}\right)-j \sin \left(\frac{\Theta \pi}{2}\right)\right]$,

where $\Theta=\frac{2}{\pi} \cot ^{-1}(\tan \delta)$. It has been previously shown that a three dimensional dispersion may adequately be represented through a two-dimensional network model[151]. The complex impedance can then be derived to be:

$$
Z(\omega)=\frac{L R^{1-\Theta}}{A(j \omega C)^{\Theta}}=\frac{L R}{A(\omega R C)^{\Theta}}\left[\cos \left(\frac{\Theta \pi}{2}\right)-j \sin \left(\frac{\Theta \pi}{2}\right)\right]
$$

While the ESR model considered a frequency dependent resistance, the CPE based representation invokes a distribution/dispersion of relaxation times, $\tau(\omega)$. Such representations have been extensively used to parameterize the frequency response of solid electrolytes [155] and solid-solid interfaces[140]. From a physical point of view, the influences of non-uniform potential and current distribution, roughness (see Section 4.8.4), composition variations, etc., are some of the parameters that could be described through such a model. The underlying idea of the $\mathrm{CPE}$ is to fit the impedance data, say that obtained through the distributed RC type model, over a defined frequency range. The constraint of a frequency range then implies that the electrical impedance, $Z(\omega)=\frac{1}{(Q \omega)^{\Theta}}\left[\cos \left(\frac{\Theta \pi}{2}\right)-j \sin \left(\frac{\Theta \pi}{2}\right)\right]$ with $Q$ as the only fitting parameter, and which can be reduced to resistive ( $\Theta \square \square \square \square)$, inductive ( $\Theta \square \square \square \square \square \square$ ), or capacitive ( $\Theta \square \square \square \square$ ) behaviors. The CPE aspect is manifested through the $\frac{Z^{\prime \prime}}{Z^{\prime}}$ ratio, from which the 
magnitude of the phase $\Theta \pi / 2$, is independent of frequency. Concomitant to the data fitting, further detailed statistical analysis may be used to indicate that the coefficient of determination [156] (i.e., the $r^{2}$ value) for the distributed models (2D RC and CPE) compared to the lumped (ESR based) model. Another important metric to describe the goodness of fit between competing models uses the Akaike Information Criterion (AIC)[157]. The underlying idea behind the AIC is that it incorporates a penalty for increasing the number of fitting parameters and consequently, a model with the minimum number of fitting parameters is preferred and given a low AIC number.

Having considered the basic methodologies for the measurement of charge transfer, the review will now proceed to a discussion of the specific electrochemical processes that lay the basis for charge storage and transfer. While metallic electrodes will constitute the first topic of discussion, later we will discuss semiconductor materials with respect to the tunability of energy levels and concomitant carrier passage from the electrode to the electrolyte.

\section{Influence of the}

\section{electrodes in charge transfer}

Charge transfer is manifested in the electrodes, electrolytes, and the electrode-electrolyte interface. In this section we focus on the electrode, with respect to the involved thermodynamics, the nature of the electrode surface, and influence of non-idealities. The material character, in terms of the charge carrier concentration, i.e., metallic or semiconductor materials, as well as the associated capacitances will be probed. This section would then serve 
as a prelude to understanding hybrid surface-bulk processes that form the basis for pseudocapacitance behavior (Section 5).

\section{1}

Charge

carrier

\section{concentration in the electrode}

We consider the nature of the electrode from the atomic scale upwards from the viewpoint of the carrier concentration in the electrodes. The gamut runs from a material with infinite conductivity (e.g., a perfect metal, which is defined as a material that does not support an electric field) to a semiconductor/ insulator. For a perfectly metallic electrode, the transfer of charges (electrons or holes) to an electrolyte would be accompanied by a surface charge layer a form of a space charge layer - see Section 4.6.1, the thickness of which is inversely proportional to the carrier concentration and hence effectively tends to zero for metals). Consequently, the carriers would ideally respond instantaneously to any change of applied potential on the electrodes. However, the charge in less perfectly conducting electrodes (as in practical metallic electrodes) is dissipated over a period referred to as the dielectric relaxation time ( $\square_{d}=\mathcal{E} / \sigma$ ), where $\mathcal{E} \square$ is the dielectric permittivity and $\sigma$ is the electrical conductivity. For example, with a Si electrode of $\sigma \square \square 10^{6} \Omega^{\square} \mathrm{m}^{-1}$ - corresponding to carrier doping of $10^{21} / \mathrm{cm}^{3}$ [19], and an $\mathcal{E}_{r}$ (see Eqn. 2) of $\sim 18[158]$ ), the $\square_{d}$ is of the order of $0.1 \mathrm{~ms}$. Such time scales would set the response of the double-layer (see Sections 2.3.1 and 2.3.2), with respect to 
its formation and dispersal, and account for the large power density of electrochemical capacitors.

It can be derived, from Maxwell relations and neglecting diffusional effects[159], that the spatial charge density, $\rho$ decays as a function of time, following the relation: $\rho(r, t) \sim \rho(r, t=0) \exp ^{-t / t_{d}}$ and implies that in a uniform electrical conductor, any uncompensated charge must accumulate at points of discontinuity such as impurities or surfaces. The screening of the electric fields/potential gradients by such charge rearrangement also occurs over an approximate length scale characterized by the Debye length, $L_{D}$ (see Eqn. 7). While the $L_{D}$ is the usual parameter considered in determining the length scale of carrier interactions, it is important to note that the derivation from the Poisson equation: $\nabla^{2} \phi=\frac{1}{\varepsilon} \rho$, where $\rho\left(=\rho_{o} e^{-\phi / k_{B} T}\right)$ is described through the Boltzmann approximation to the Fermi-Dirac distribution, and assumes that the potential, $\phi \square<<k_{B} T$ ( $26 \mathrm{meV}$ at room temperature of 300 K), which may not always be true. Larger $\square \phi$ values demand that the full Fermi-Dirac distribution be taken into account, yielding the Thomas-Fermi screening length, where $k_{B} T$ is replaced by the $E_{F}[160]$. However, $L_{D}$ continues to be used widely and is quite appropriate for non-degenerate semiconductors with smaller values of the $\rho$. Generally, larger values of $L_{D}$ imply larger length scales where carrier interactions ought to be considered along with larger time scales of charging/discharging the formed EC. While the $L_{D}$ construes the screening of the charge on the electrode to take place in a continuum, the discreteness of the electrolyte in terms of the cations, anions, and the electrons is better understood through another overlapping length scale, i.e., the thickness of the diffuse double layer (Section 2.3.2). Other length scales have also 
been invoked to describe the various species and mechanisms involved in charge transfer. For example, a distinction between free, bound, and adsorbed ions could be made on the basis of the $L_{D}$ as well as the Bjerrum length $\left(L_{B}\right)$ [161]. At distances less than that of the $L_{B}$ the ions can be considered bound as the relevant electrostatic energies are less than $k_{B} T$ and randomizing/Brownian motion could be reduced. On the other hand, at length scales $>L_{D}$, the ions can be considered free as this length scale governs the radius of the oppositely charged, screening, atmosphere that surrounds a particular ion.

\section{The thermodynamics}

\section{of charge transfer in a redox couple}

The screening and the formation of the double layer (Section 2.3.1) affect and mediate the charge transfer at the electrode-electrolyte interface. For a metallic electrode, the carrier (electron/hole) transfer between the energy levels in the solid and the redox levels in the electrolyte proceeds through quantum mechanical tunneling processes, as discussed in the next section. The exponential correlation of the electrochemical reaction rate constant with the applied potential (see Eqn. 19) lends credence to such a mechanism. The Franck-Condon principle, whereby the electron movement corresponding to the redox reaction occurs at time scales much shorter compared to the atomic/ionic movement, is invoked in the charge transfer in the redox couple which may be present in the electrolyte. The energy scales and relative separations of the oxidized $(O)$ and reduced $(R)$ species can be understood qualitatively through the energy-reaction coordinate (q) diagrams as in Figure 23, where the Gibbs 
free energies $(G)$ are indicated and $\mathrm{q}$ refers to the average spatial separation between the moieties. The fundamental tenet of electron charge transfer may be interpreted in terms of a single electron oxidation/reduction reaction of the form: $O+e^{-} \rightarrow R$ (as in Eqn. (2) with $n=1$ ). Considering $\mathrm{q}_{\mathrm{ox}}$ and $\mathrm{q}_{\mathrm{red}}$ as the equilibrium (and minimum) values relevant to the $O$ and $R$ species, the energy curves are represented in parabolic form (implying a Hooke's law formalism for the constituent forces). Two important features in such diagrams are: (i) the relative displacement of the $O$ and $R$ energy curves (i.e., $\mathrm{q}_{\mathrm{red}}>\mathrm{q}_{\mathrm{ox}}$ ), which may be understood through a corresponding greater equivalent volume (due to the addition of the electron) for the $R$, and (ii) that the free energy curve for $R$ is lower than that for the $O$, implying that the redox conversion from $O$ to $R$ is energetically allowed and thermodynamically feasible.

Generally, a larger displacement $\left(=\left|\mathbf{q}_{\text {red }}-\mathbf{q}_{\mathbf{o x}}\right|\right)$ would imply a greater barrier to interconversion and in the language of reaction kinetics - a higher energy for the transition state in between the product and the reactant. The implication of such a shift is manifested in the case of an electron addition to $O$ at $\mathrm{q}_{\mathrm{ox}}$ through the electron affinity (E.A.) energy. Due to the $R$ energy curve displacement, excess energy corresponding to the energy difference to the minimum of the curve (i.e., $\left.G_{r e d}^{0}\right)$ is dissipated and relates to reorganization energy $\left(\lambda_{\text {red }}\right)$. Similarly, the removal of an electron from $R$ at $\mathrm{q}_{\mathrm{red}}$ (which yields $O$ ) implies an ionization energy (I.E.), with the corresponding energy difference to the $O$ energy curve minimum (i.e., $G_{o x}^{0}$ ) being dissipated and relating to the re-organization energy $\left(\lambda_{o x}\right)$ of the $O$ species. The difference: $G_{o x}^{0}-G_{r e d}^{0}$ corresponds to the Fermi energy $\left(E_{F}\right)$, as the energy involved in filling the $\mathrm{O}$ species or as the energy related to the electron in the system. It is noted that the one-electron approximation for 
the solid as typical to solid state physics[160] is being employed. Multiple electron transfers are considered as a succession of one-electron events, or reduced to a simplified form, where the rate determining one-electron transfer is dominant[124] see Eqn. 2' in Section 3.1.2.

The physical significance of the $\lambda$ is then further understood in terms of the energy difference between the $R$ and the $O$ energy levels at equilibrium, i.e., as related to the reorganization in the moiety involved in removing/adding one electron to the respective moieties. Thus far, the equilibrium situation considering the energy minimum has been discussed. In the more general case of far from equilibrium, with values of $\mathbf{q}$ away from their minimum values, the difference of the $O$ and $R$ energy curves (say, $G_{o x}$ and $G_{r e d}$ ) yields the energy (or equivalently the electrode potential) of the redox system. Considering thermally activated population of the higher energy levels, the probability of occupation $(W)$ would be proportional to $\exp \left(-\frac{\Delta G}{k_{B} T}\right)$ where the $\Delta \square G$ is the free energy difference between the equilibrium level and the pertinent energy level. As the energy curves were assumed to be parabolic, e.g., $G_{o x}$ $\sim\left(\mathrm{q}-\mathrm{q}_{\mathrm{ox}}\right)^{2}$; assuming a correspondence of the $\mathrm{q}$ values to the relevant energies, i.e., $\mathrm{q}-\mathrm{q}_{\mathrm{ox}}$ equivalent to an energy difference, $\Delta \square E_{o x}$ and scaling by $\lambda$, we get $\Delta G_{o x} \sim \frac{\Delta E_{o x}^{2}}{\lambda}$. Similarly, $\Delta G_{r e d} \sim \frac{\Delta E_{r e d}^{2}}{\lambda}$. The $W$, which would correspond to a probability distribution function, are written as: $W_{o x} \sim \exp \left(-\frac{\Delta E_{o x}^{2}}{\lambda k_{B} T}\right)$ and $W_{\text {red }} \sim \exp \left(-\frac{\Delta E_{r e d}^{2}}{\lambda k_{B} T}\right)$, for the $O$ and $R$ species and signifies the number distribution of allowed states in the redox couple with the $O$ and the $R$. The bell-shaped curves for $W_{r e d}$ and $W_{o x}$ - see the shape of $\mathrm{W}(\mathrm{E})$ in the middle of Figure 24 follow from such a description, with the centers at $E_{r e d}^{o}$ and $E_{o x}^{o}$, respectively and half-width corresponding to the 
respective $\lambda$ values. The Fermi level of the redox couple $\left(E_{F, \text { redox }}^{0}\right)$ is considered to be intermediate between the energy levels of the $R$ and the $O$ species, i.e.,

$$
E_{F, \text { redox }}^{0}=\frac{E_{r e d}^{0}+E_{o x}^{0}}{2} \text { with } E_{r e d}^{0}=E_{F, \text { redox }}^{0}+\lambda_{r e d}, \text { and } E_{o x}^{0}=E_{F, \text { redox }}^{0}-\lambda_{o x}
$$

A more formal derivation of the above can be seen, for example, in Section VIII.A of Reference[81]. An alternate theoretical methodology - the Marcus theory of electron transfer, as briefly described in Ref. [124], has also been invoked to describe the $O\langle->R$ conversion taking a chemical kinetics point of view of a reactant to product conversion, involving an intermediate transition state. The Marcus theory does not consider then the energy resonances involved in electron transfer (i.e., no interaction between energy levels) and predicts for example, a charge transfer coefficient (e.g., $\alpha$ ) - see Eqn. (19) and related discussion (Section 3.1.2), that varies as $0.5(1+\Delta G \square \lambda \square \square \square$ i.e., that $\alpha=0.5$ at equilibrium when $\Delta G=0$, and that $\alpha \rightarrow 1 / 0$ for cathodic/anodic biases, where $|\Delta G| \sim \square \square$. However, such a classical transition state theory is not accurate in the presence of strong coupling (an adiabatic limit) where energy quantization and resonances do have to be considered. Indeed the interaction between the energy levels of the electrode with those of the electrolyte may give rise to an energy spread $(\Delta)$ that can be much greater than $1 \mathrm{meV}$ and a non-adiabatic/interacting approximations may be invalid. Moreover, the Gerischer diagram based formulation (as in Figure 24) of electrode-electrolyte interaction assumes a Lorentzian DOS while the Marcus transition state based theory has a sharper cut-off for the $O$ and $R$ species through the use of a Gaussian DOS. While most experiments to-date do not clearly distinguish between the Marcus and the Gerischer models they are also not adequate to probe the differences arising from the electron transfer rate, which needs very large scan rates 
$(s)$ and relative neglect of diffusion based processes, in cyclic voltammetry (see Section 3.1). The classical foundation of the Marcus transition state based theory also implies a thermally activated process, while the Gerischer model assumes tunneling for the charge transfer- an inherently temperature independent process. An excellent first introduction to the electrode-electrolyte interface, from such a point of view, as well as the relevant principles of electrochemistry has been given in Gerischer's monograph[81] While the Gerischer model looks appealing, it predicts a rate constant for charge transfer that increases exponentially with distance - contrary to present experimental observations[162]. While additional polarization sensitivity could be invoked, experiments to probe kinetics in the absence of possible reorganization of the reacting species may be necessary.

The notion of electrode-electrolyte coupling and the subsequent agility of the redox reaction may also be classified through the nature of the electrolyte. When the interaction between the $O$ and the $R$ species (or alternatively between the donor or acceptor) is weak, the coupling is termed non-adiabatic and the $O \leftrightarrow R$ conversion is driven by the free energy of reaction as given in the introductory expositions of Marcus[163,164]. Termed as an outer sphere electron transfer, such a classical approach of calculating the energies was adequate as the activation barrier for the electron transfer was posited to occur due to the differences in the nuclear configurations of the reactants and products[165]. The intuitive basis for such an assertion the relative response times of the contributors to the overall electrical polarization, i.e., electronic (of the order of $10^{-15} \mathrm{~s}$ ), atomic $\left(\sim 10^{-13} \mathrm{~s}\right)$, and the orientational $\left(\sim 10^{-11} \mathrm{~s}\right)$, whereby the electron transfer can by itself be considered adiabatic and the overall rate constant was determined by the slower polarization mechanisms[163]. As the electron transfer in such mechanisms does not involve a change in the bonding configuration and is outwardly equivalent to the addition of an electron to a moiety, the 
term outer sphere electron transfer is used. It is noted that such non-bond induced electron transfer depends on the reorganization energy $(\lambda)$ of the $R$ and the $O$ species, which strive to reach their lowest energy configurations, see for example Figure 23, through nuclear rearrangements, bond length adjustment, etc. Such a mechanism is dominant in the redox reactions between species whose inner coordination spheres (e.g., constituted from solvated ions) remain intact during electron transfer, such as the reaction:

$$
\mathrm{Fe}\left(\mathrm{CN}_{6}\right)^{3-}(a q)+\mathrm{Fe}\left(\mathrm{CN}_{6}\right)^{4-}(a q) \rightleftharpoons \mathrm{Fe}\left(\mathrm{CN}_{6}\right)^{4-}(a q)+\mathrm{Fe}\left(\mathrm{CN}_{6}\right)^{3-}(a q)
$$

Typically, the presence of bulky surrounding ligands promotes such a charge transfer and the outer Helmholtz plane (Section 2.3.1) determines the locus of the reacting centers. Reactions in nature, which demand specific and relatively rapid reaction times, offer further examples of outer sphere transfer. Alternatively, inner sphere/bonded electron transfer involves electron sharing, e.g., through a common ligand between the $R$ and the $O$ species. The involvement of an intermediate ligand results in slower charge transfer kinetics.

\section{between the electrode and the electrolyte}

Superposing energy level diagrams (with the vertical axis in terms of the energy and the horizontal axis akin to a density of states) the charge transfer between the metal electrode and the electrolyte can be visualized, as in Figure 24. At equilibrium, the Fermi level of the metal $\left(E_{F, \text { metal }}^{0}\right)$ coincides with the energy of the redox couple $\left(E_{F, \text { redox }}^{0}\right)$ after the energy involved in the dipole formation (i.e., constituted from the negatively 
charged electrons in the metal and the positively charged ions in the electrolyte) is taken into account. If the dipole lowers the energy for the ionization by $e \varphi$ then, $E_{F, \text { metal }}^{0}-e \varphi=E_{F, \text { redox }}^{0}$. The figure also indicates that under such a condition of equilibrium, the cathodic current: $i_{c} / i_{+}$(due to electron transfer to the electrolyte from the electrode) is the same as the anodic current: $i_{a} / i$. (due to electron transfer from the electrolyte to the electrode) and termed as the exchange current ( $i_{o}$ - see Section 3.1.2 and Eqn. 21). Aspects related to cathodic (/anodic) polarization whereby the applied voltage is negative (/positive) and the Fermi level shifts up (/down) are abundantly clear from such "Gerischer diagrams". The excess voltage over the equilibrium is termed the overpotential ( $\square \square$. The probability of electron transfer between the electrodes and the electrolyte is augmented, in either the cathodic or the anodic cases, by the $\eta$ and results in an enhancement of the exchange current (as indicated in the rightmost diagram of Figure 21.

The enhancement is indicated through the Butler-Volmer expression for the total current, as:

$$
i=i_{c}-i_{a}=i_{o}\left\{\exp \left(-\frac{\alpha F}{R T} \eta\right)-\exp \left(-\frac{(1-\alpha) F}{R T} \eta\right)\right\}
$$

This expression is to be compared to the one previously stated as Eqn. (21) - in Section 3.1.2. The $\alpha \square$ and the (1- $\alpha)$ are referred as the anodic and the cathodic charge transfer coefficients for a one-electron transfer reaction. It is also assumed that the coupling between the electronic states in the metal and states in the electrolyte are sufficiently strong and predominate over mutual rearrangement of charge in the respective electrode or electrolyte. Further details concern taking into account the interaction strength between the electronic states of the electrode as well as 
those of the electrolyte, considering the appropriate perturbation theory and where the transition rate (and the reaction constant) can be estimated through the Fermi-golden rule[129]. While the supply of species to the metal electrode is not considered to be a rate-determining factor, diffusion based limitations are taken into account through modifications to the Butler-Volmer equation, e.g., through the ratio of the actual number of species $\left(N_{\text {red }}\right.$ or $\left.N_{o x}\right)$ to those at equilibrium/when such diffusion limitations are absent $\left(N_{\text {red }, 0}\right.$ or $\left.N_{o x, 0}\right)$ as follows:

$$
i=i_{c}-i_{a}=i_{o}\left\{\frac{N_{r e d, 0}}{N_{r e d, 0}} \exp \left(-\frac{\alpha F}{R T} \eta\right)-\frac{N_{o x, 0}}{N_{o x, 0}} \exp \left(-\frac{(1-\alpha) F}{R T} \eta\right)\right\}
$$

The ratio $N_{\text {red }} / N_{\text {red }, 0}$ or $N_{o x} / N_{o x, 0}$ provide a further measure of the concentration polarization (see Section 2.2 and Figure 2). The physical mechanisms underlying the charge transfer are related to the quantum mechanical tunneling (see Figure 25) between levels at the same energy (charge moving from an occupied state to an empty state) separated by an energy barrier (corresponding to the sum total of the energies involved in the dipole formation, species reorganization, thermal activation, etc.). Consequently the potential drop (related to the electrochemical potential difference) occurs over the tunneling distance of $\sim 1 \mathrm{~nm}$ or less. The typical use of a high concentration (> $0.1 \mathrm{M}$ ) supporting electrolyte ensures the efficiency of such electrode kinetics.

\section{Drift-Diffusion based processes}


The essential elements underlying charge storage and transfer involve the interaction of ions in the electrolyte with the electrode through either diffusion to the surface and subsequent electrochemical reaction or the latter alone (as in the thin layer electrochemistry case: see Section 5.2). The forces [166] driving such a reaction arise due to a concentration gradient or electric field, respectively. While the double layer capacitance, $C_{d l}$ is independent of time and voltage (at sufficiently large positive or negative voltages - see Sections 2.3.1 and 2.3.2), the additional measured capacitance (which would be a function of time over which the potential changes by $d V$ ) and the associated charge transfer would be proportional to $d q / d V$ (also see Section 5.1). From elementary considerations[79], the measured current is linearly proportional to the scan rate, s, i.e.,

$$
i_{p}=\frac{d q}{d t}=\frac{d q}{d V} s
$$

However, a charge concentration gradient can give rise to an electrical current as well and the consequent diffusion current is manifested through the Randles[132]- Ševčík[133]equation[79], as related to Eqn. (15) in Section 3.1.2 and reproduced below:

$$
i_{p}=0.4463 \sqrt{\frac{F^{3}}{R T}} z^{3 / 2} A D_{o}^{1 / 2} I_{o} s^{1 / 2}=2.69 \cdot 10^{5} z^{3 / 2} A D_{o}^{1 / 2} I_{o} s^{1 / 2}(\text { at } 298 \mathrm{~K})
$$

The proportionality of the current to $\sqrt{s}$ distinguishes the contribution of diffusion-based processes from surface oriented redox processes, which contribute to the pseudocapacitance (where the current is proportional to $s$ ). However, as diffusion must be accompanied by an oppositely directed drift [167], the concurrent process must also yield a $\sqrt{s}$ dependence. It can be shown that such drift currents would dominate the diffusion currents, and further enhance the 
electrical current densities that may be obtained from an electrochemical device. We consider an electrode with a net charge density (charge/per unit volume) of $Q_{m}$ in contact with an electrolyte of total charge density, $Q_{i o n}$, as in Figure 26 (a).

While the formation of an electrical double layer[79] on the surface of an electrode, due to charge compensation or screening from the electrolyte is reasonably well understood (see Section 2.3.1), the spatial distribution of the ions may be quite complex. We examine a simplified version where the ions do not perfectly screen the electrode charge (i.e., $Q_{i o n}<Q_{m}$ ) and a one-dimensional charge variation. We assign the spacing between the electrodes to be $L$, and that between the edges of the double layers to be $L^{\prime}$, implying that the thickness of the double layer $\left(t_{d l}\right)$ on an electrode would be on the average, equal to $\left(L-L^{\prime}\right) / 2$, with $2 t_{d l}<<L$.

The electric field $(\xi)$ variation in the device $(0<x<L)$ in both the double layer $\left(E_{d l}\right)$ and the electrolyte $\left(E_{e l}\right)$, as in Figure 26(b) can be obtained from: $\xi=\int_{0}^{x} \frac{\rho}{\varepsilon} d x$, with $\rho$ as the respective charge density $\left(=Q_{m}\right.$ or $Q_{i o n}$ ) with $\varepsilon$ being the equivalent and uniform dielectric permittivity[167]. When $Q_{i o n}$ is only slightly smaller than $Q_{m}$, the $\xi_{d l}$ would be significantly larger than the $\xi_{e l}$. Further spatial integration leads to the potential distribution, $V(x)=-\int_{L}^{x} \xi d x$, with the potential of the counter electrode set to a reference value of zero, as in Figure 26 (c). Consistent with $\xi_{d l}>\xi_{e l}$, the voltage across the double layer, $V_{d l}$ would be significantly larger than that across the electrolyte, $V_{e l}$. It is to be noted that an applied voltage across the electrodes/device, $V_{a p p}=2 V_{d l}+V_{e l}$. As the charge distribution in the double layer is related to the electrolyte ion radius and to thermal fluctuations and occurs over spatial scales typically several orders of magnitude smaller than the device length, there is no appreciable difference 
between representing the double layer to be of a finite width $v s$. that of a sharply peaked distribution, for the purposes of partitioning the $V_{a p p}$. However, the shape of $Q_{i o n}$ in Figure 26 (a) reflects the fixed electrical double layer closer to the electrode - reckoned with respect to the outer Helmholtz plane (Section 2.3.1), and the adjacent diffuse double layer with an exponential decay in the ion concentration[79]. We define $\gamma$ as a measure of the screening strength[160] of the electrode charge through:

$$
\gamma=\frac{V_{e l}}{V_{a p p}}
$$

Considering that $V_{a p p} \approx 2 t_{d l} E_{d l}+L^{\prime} \xi_{e l}, V_{e l} \approx L^{\prime} \xi_{e l}, \xi_{d l}=Q_{m} / \varepsilon$, and $\xi_{e l}=\left(Q_{m}-Q_{i o n}\right) / \varepsilon$, then:

$$
\frac{Q_{i o n}}{Q_{m}}=1-\frac{2 t_{d l}}{L^{\prime}} \frac{\gamma}{1-\gamma}
$$

In this form, with $\gamma=0$ we get $Q_{i o n} / Q_{m}=1$, implying that the double layer holds exactly as much charge as the electrode and we have perfect screening; there would no driving force for an electrochemical redox (reduction-oxidation) reaction in this case. With $\gamma=L^{\prime} / L, Q_{i o n} / Q_{m}=0$, meaning that there is no screening whatsoever of the electric field from the double-layer to the bulk electrolyte. Note that $\gamma$ cannot be equal to 1 in this formulation. We now study the behavior of ions in the electrolyte under the influence of $\xi_{e l}$, which induces a drift velocity, $\dot{x}=\mu \xi_{e l}$, with $\mu$ being the ion mobility. The electrical current density (in units of current/area) $J=q\left(I \cdot N_{A}\right) \dot{x}$, with $q=z e$ ( $z$ is the charge per ion, $e$ is the elementary electronic charge), $I$ is the bulk electrolyte concentration in moles/liter, and $N_{A}$ is the Avogadro number. We regard a typical experiment in electrochemistry, e.g., CV (as described in Section 3.1) which can determine the complete electrochemical behavior of a system[79] and is often used to monitor the nature and reversibility 
of the cathodic and anodic reactions in an electrolyte[124,169]. CV involves a linear sweep of the $V_{a p p}$ and recording the net electrical current through the electrodes. The rate of change of the voltage, the scan rate, $v=d V_{a p p} / d t$ can be related to $\xi_{e l}$ using Eqn. (38) by:

$$
\xi_{e l}=\frac{d V_{e l}}{d x}=\frac{d\left(\gamma V_{a p p}\right)}{d x}=\gamma \frac{d V_{a p p} / d t}{d x / d t}=\frac{\gamma s}{\dot{x}}
$$

It is assumed that the $\gamma$ is spatially independent, since neither $V_{e l}$ nor $V_{d l}$ are spatially dependent. Then, $\dot{x}=\mu \xi_{e l}=\mu \frac{\gamma \nu}{\dot{x}}$, so that $\dot{x}=\sqrt{\gamma \mu \nu}$. When substituted into $J=q\left(C N_{A}\right) \dot{x}$ and through use of the Einstein relation $\mu \square=(z e) D / k_{B} T$ and the equality $F / R T=e / k_{B} T$ ( $D$ is the diffusion coefficient) yields:

$$
J=\sqrt{\gamma} \cdot I \sqrt{F^{3} \frac{D s}{R T}} z^{3 / 2}
$$

Such a form for the current density has been previously established through invoking elaborate semi-infinite diffusion based techniques and cited in literature[79,80,141] as the Randles- Sev̂cik equation: $J=a \cdot I \sqrt{F^{3} \frac{D s}{R T}} z^{3 / 2}$, with $a$ being a numerical constant - see Eqn. (15). Such a relation was defined by mapping the redox reaction current to a diffusion current anchored to the electrode surface. The device operates in a closed circuit, so the currents thus defined at one surface are related to the currents at the opposing surface. It is possible to find an analytical integral equation for device current as a function of time, $i(t)$. The normalized peak current[79] incorporates an $a=0.4463$ - see Eqn. (15) in Section 3.1.2; the corresponding peak current density is hereby defined as $J_{\text {peak }}$. Therefore, there is no empirical parameter involved in $a$. It has now been shown that electric field induced drift in the bulk electrolyte could yield a similar form 
for $J$, reinforcing the deep connection between drift and diffusion. Assuming an equivalence of our derived relation - Eqn. (42), with the Randles-Sev̂cik equation, we can now assign a physical significance for the constant, $a$ :

$$
\sqrt{\gamma}(\text { proposed drift model })=a(\mathrm{R}-\mathrm{S} \text { equation })
$$

For example, a match of the R-S equation's $a$ coefficient in the $J_{\text {peak }}$ of 0.4463 with $\sqrt{\gamma}$ leads to a $\gamma \sim 0.2$. In general, a larger value of $\gamma$ corresponds to a larger value of $V_{e l}$, although it is limited by the $V_{a p p}$ as depicted in Figure 27.

Equivalently, $J$ increases if screening can be weakened without affecting the other factors in Eqn. (42). The imperfect screening of $Q_{m}$ by $Q_{i o n}$ causes $V_{e l}$ to be a fraction of $V_{a p p}$. At larger the screening is further reduced since the ions cannot respond to the quicker change in $V_{a p p}$, resulting in a larger $E_{e l}$ and a larger ionic current density. Generally, the electric field induced drift current density $\left(J_{\text {drift }}\right)$ could be assumed to be dominant over the diffusion current density $\left(J_{\text {diff }}\right)$ from the bulk electrolyte, i.e., outside the double layer. This can be understood by taking the ratio of $J_{d r i f t}=q\left(I \cdot N_{A}\right) \mu \frac{d V_{e l}}{d x} \approx q\left(I \cdot N_{A}\right) \mu \frac{\gamma V_{a p p}}{L^{\prime}}$ to $J_{\text {diff }}=q D \frac{d\left(I \cdot N_{A}\right)}{d x} \approx q \frac{k_{B} T}{q} \mu \frac{I \cdot N_{A}}{L^{\prime}}$ which is equal to $\gamma \frac{V_{a p p}}{k_{B} T / q}$. At $T=300 \mathrm{~K}$, (with $k_{B} T / q \sim 26 / z \mathrm{mV}$ ) and assuming a $V_{a p p}=1 \mathrm{~V}$ and $z=1$, we get $J_{\text {driff }} / J_{\text {diff }}=40 \gamma / z$. For electrolytes as $\mathrm{K}_{3} \mathrm{Fe}(\mathrm{CN})_{6}$, with $z=1$ for the positive ions and 3 for the negative ions. If $\gamma \square \square \sim 0.25$, as indicated and also justified later in the paper, then $J_{\text {driff }} / J_{d i f f}=$ $10 / z \gg>1$. As a result, for the assumed bias conditions, basing the total current on drift current is appropriate. The above formulations and conclusions apply to both positive and negative ions, albeit with different $\mu$ and $z$. 
Comparison of the developed drift model to experimental current densities was used for additional insight into the nature and variation of $\gamma . J v s . I \sqrt{s}$ was plotted from experimental data for both flat Pt electrodes and for nanostructured CNT electrodes (constituted of CNTs with average length, diameter, and separation of $25 \pm 4 \mu \mathrm{m}, 20 \pm 5 \mathrm{~nm}$ and $150 \pm 50 \mathrm{~nm}$ electrode[170] ) in Figure 28. Empirically, $J=0.674 I \sqrt{s}$ for Pt and $J=0.786 I \sqrt{s}$ for CNT. Assuming the same diffusion coefficient $D=6.8 \times 10^{-6} \mathrm{~cm}^{2} / \mathrm{s}$ for both positive and negative ions[79] at $T=300 \mathrm{~K}$, from Eqn. (42) we have $\sqrt{F^{3} \frac{D}{R T}}=1.56\left[\frac{\mathrm{mA} / \mathrm{cm}^{2}}{\mathrm{mM} \sqrt{\mathrm{V} / \mathrm{s}}}\right]$. Therefore, $J=$ $\sqrt{\gamma} 1.56 I \sqrt{s}$ for both Pt and CNT. For Pt, $\sqrt{\gamma} \sim 0.4327$ and thus $\gamma \sim 0.2$, in close accord to the theoretical value of $a=0.4463$ as in Eqn. (42). However, for CNT, $\sqrt{\gamma} \sim 0.5045$ and thus $\gamma \sim$ 0.25. The experimental procedures as well as the relevant parameters[171] and data for the fitting were taken from the papers by Hoefer, et al[170,172].

A greater value of $\gamma \sim 0.25$ for the CNT electrodes compared to $\gamma \sim 0.2$ for Pt electrodes indicates that the screening of the electrode has been weakened, allowing a larger electric field in the electrolyte. The consequent larger magnitude of the $E_{e l}$ and the $J_{d r i f t}$ could account for the larger current densities of Figure 28, for the nanostructured electrodes. The close spacing of the CNTs could result in a relative starvation of the screening ions from the electrolyte yielding a greater $\gamma$, and suggests design methodologies to further increase electrical current densities.

In summary, such a treatment seems to indicate the principles through which the net current density of electrochemical devices could be enhanced by harnessing additional ionic current from the bulk electrolyte. A metric $(\gamma \sqsupset)$, related to the ratio of the potential drop in the electrolyte to that of the applied voltage, has been introduced through which the current density 
increase (in terms of $\sqrt{\gamma}$ ) may be parameterized. Further optimization of the nanostructured electrode electrochemical capacitors, based on the fact that the theoretical upper limit for $\sqrt{\gamma}$ approaches unity, would be worthy of continued study.

The relation of the current $(i)$ to the $(s)$, through the exponent, $b$, in $i=a s^{b}$ has been considered as the basis for relating the extent to which diffusion based $(b=0.5)$ or surface based $(b=1)$ processes dominate in a particular electrochemical process. For example, an intermediate value of $b \square \sim 0.7$ was ascribed to Ohmic losses[173], whereby if the diffusion limitations were removed would have yielded a $b=0.5$. Such interpolations may not be valid as they imply that there is somewhat a smooth gradation between diffusion-limited processes and surface limited processes. As we have shown earlier, either drift or diffusion could give identical results and stand distinct from say, surface limited processes. Generally, when $i=C s$, it is implicitly assumed that the capacitance is not time independent or that close to equilibrium conditions are present, i.e., that at a given $s$, all the species that could contribute to the capacitance at a given time are present. When there are limitations due to diffusion, $i=C s+V \frac{d C}{d t}$ at a given potential, say for the redox reaction and the second term yields a fluctuating capacitance value. Further, there would be a capacitance gradient as can be surmised from: $i=C s+V \frac{d C}{d x} x$, where the species velocity $(=x)$ and mobility could play a role. It can then be reasonably concluded that the surface processes could be described through a linear relationship between $i$ and $v$, with the $v=0$ intercept indicating the departure from equilibrium conditions. 


\section{state of an electrode, e.g., the SEI (Solid Electrolyte Interface)}

The difference in the electrochemical potentials of the electrode (anode/cathode) with respect to the electrolyte was previously discussed (in Section 2.2 and Figure 1) and could promote the adsorption of a minor phase onto an electrode. The rapid formation of a surface layer, which has been termed as a Solid Electrolyte Interface (SEI) [174] affects the charge transfer kinetics in practical electrochemical systems, as the adsorbed product may not always be electrochemically active. When the SEI thickness becomes larger than the electron tunneling distance, of the order of 1-2 nm, the electrode performance as related to the charge transfer (see Section 4.3) would be severely compromised. The SEI could be modeled through a phase field method[175,176], which considers a diffuse interface, typical of the SEI, between the electrode and the electrolyte, and is based on analogies of the interface to a solid-melt interface. The charge in the SEI interface is assumed to be confined/localized to a smooth interface, the distribution of which can be dynamically altered through the passage of an electrical current. Generally, during oxidation (e.g., during discharge of the battery) the attraction and the indiffusion of the anions in the electrolyte to the positive anode enhances the SEI thickness. On the negative cathode (which in the case of $\mathrm{Li}$ ion batteries, is typically composed of $\mathrm{Li}$ - transition metal based oxides) the inward diffusion of the $\mathrm{H}^{+}$ions and the consequent ion exchange induced may lead to anodic shifts in the delithiation processes[177]

Such SEI related issues are particularly deleterious for Lithium ion batteries, where with the presence of complex electrolyte systems (Section 2.2.3) the formation of intermediate metastable compounds on both the anode and the cathode (Section 2.2.2) would be expected. 
While the tendency for SEI formation would be reduced if the electrochemical potentials of the electrodes were situated within the electrolyte LUMO-HOMO window - see Figure 1, this would occur at the expense of a reduction in the operating voltage as well as the energy density. Alternately, the interface has the practical effect of increasing the electrical resistance (reported to be in the range of $10-100 \Omega \mathrm{cm}^{2}$ and sometimes as high as $10^{8} \Omega-\mathrm{cm}^{2}$ ) as well as the mass transfer resistance (Section 3.3) and results in reduced electronic and ionic conductivity [178]. The effects on kinetic parameters (e.g., the diminished/unequal reduction-oxidation transfer coefficients in the Butler-Volmer equation[79] - see Eqns. (20), (21), and (36)) as well as the lifetime of the electrochemical device (e.g., due to the loss of Lithium capacity due to agglomeration in the anode, the formation of dendrites, reduced capacitance, etc.) have all been implicated to the SEI. Similar interface formation has been noted in other electrode systems as well, e.g., in noble metal systems and in nanostructured electrodes (such as CNTs) - where the SEI constitutes a larger fraction of the electrode size.

The mechanisms associated with SEI formation can be studied in situ through electrochemical impedance spectroscopy (EIS) [139] using Nyquist plots (Section 3.3), where the various time constants associated with voltage independent surface film formation, voltage dependent charge transfer, ion diffusion through the SEI, and Li accumulation may all be mapped out. In this context, many possible electrode-electrolyte solution interactions which may influence the device performance through SEI formation has been previously reviewed[179]. Additionally, ex situ x-ray photoelectron spectroscopy (XPS), atomic force microscopy (AFM), and Fourier transform infrared spectroscopy (FTIR) observations indicated the: (a) formation of a porous structure through reaction and replacement of the surface films, and (b) an oxygen content/oxidation state increasing outwards from the anode. While some of the 109 
intermediate/metastable Li based compounds may serve as $\mathrm{Li}$ ion conductors, it is feasible that the inability of such compounds to reorganize through electrochemical processes coupled with porosity may lead to the formation of Li dendrites.

As the SEI formation seems inevitable, methodologies to provide a stable SEI layer have been advocated through the use of additives, such as ethylene carbonate which has been shown to form a stable passivating film on a graphite anode surface. Such stability or the ability to rapidly heal a disrupted SEI is necessary to avoid Li plating and dendrite formation. Further dendrite elongation would be driven through a concentration gradient between the electrode and the electrolyte, leading to non-uniform current distribution and ultimately device failure when the dendrites electrically short to the other electrode[180] and lead to catastrophic failure of the battery. Generally, such problems are exacerbated at lower temperatures and slower discharge.

Indeed, it has been considered that the rate of healing of the SEI layer is another determinant of the safe rate of charging of a graphite anode. Alternatively, the use of intrinsically disordered graphitic carbons, which may be relatively insensitive to volume changes, would be attractive. Additionally, the unintended storage of $\mathrm{Li}$ in the SEI would also contribute to a diminished energy density. It has been estimated that with graphite anode, up to $20 \%$ of the $\mathrm{Li}$ may be consumed to form the SEI, implying a corresponding rise in the deadweight of the anode[181] and a decrease in the energy storage capacity. Interesting methodologies to compensate for the Li loss to the SEI have been proposed, involving pre-lithiation of the anode[182] as well as the use of sacrificial salts[181]. In the latter approach, a Li salt with an oxidizable anion, e.g., $\mathrm{LiN}_{3}$, is intermixed into the graphite anode. In the initial charging process, the anion is converted to a gas (through loss of electrons), e.g., the $\mathrm{N}_{3}{ }^{-}$is converted to $\mathrm{N}_{2}$, which 
may be removed while the Li stays behind. It was also proposed that the gas conversion would have the beneficial effect of introducing porosity into the electrode (Section 4.9), which in addition to increasing the effective rate of charge transfer could also serve for electrolyte storage. The sacrificial salts are chosen so as to decompose in a potential range corresponding to the voltage capacity of the anode, e.g., at $\sim 4 \mathrm{~V}$, for $\mathrm{LiMn}_{2} \mathrm{O}_{4}$ or $\mathrm{LiCoO}_{2}$.

Purposeful deactivation or coating of the electrode/electrolyte interface with an SEI layer is also being actively pursued. To this end, coatings of inert materials such as $\mathrm{Al}_{2} \mathrm{O}_{3}$ or $\mathrm{ZnO}$ have been deployed, and may also have the desirable effect of reducing the loss of oxygen and lithium ion vacancies mitigating the irreversible capacity loss. However, maintaining good electronic contact of the electrode material with the current collector would be necessary. Alternately, use of less electroactive species, e.g., involving species such as $\mathrm{Fe}^{3+}, \mathrm{Cr}^{3+}$ or $\mathrm{Ga}^{3+}$ on the surface of the cathodes, have been shown to improve the chemical stability, as well as suppress the SEI[183]. It has also been indicated that chemical treatment of the carbon anodes with $n$-butyl lithium may have the effect of aiding SEI formation while eliminating the sources of irreversibility associated with oxide or carbonyl groups [184]. However, the resultant larger thickness of the SEI and brittleness diminishes such gains. As the porosity coupled with relative mechanical rigidity of the SEI could be deleterious, it was suggested that suitable stabilization could be achieved through the formation of flexible, elastomeric films, which could accommodate the volume changes due to $\mathrm{Li}$, compound formation and associated electrochemical processes. For example, the use of diblock or triblock polymers, e.g., of the form poly(styrene-block-ethylene oxide) (SEO) was advocated[185] whereby the ethylene oxide domain allows for higher ionic conductivity while the more mechanically stiff styrene-based domains allow for structural stability as well as disrupting the SEI and dendrite formation, as in 
$\mathrm{Li}$ ion batteries. As another example of such usage on $\mathrm{Si}$ anodes, where the SEI is even more tenuous compared to that on graphite, is the use of at least a $50 \mathrm{~nm}$ thick Lipon (lithium Phosphorus oxynitride) film coating for an artificial SEI[186]. The Lipon coating works as a physical barrier to electrode-electrolyte contact as well as to transport the $\mathrm{Li}$ ions through while blocking the electrons. The respective electronic and the ionic conductivity were measured to be $\sim 10^{-13} \Omega^{\square \square} \mathrm{cm}^{-1}$ and $\sim 10^{-6} \Omega \square \mathrm{cm}^{-1}$, respectively, addressing the need of the coating as an excellent ionic conductor while serving as a poor electronic insulator.

\section{Charge transfer in}

\section{semiconductors}

While metallic electrodes with very large carrier concentrations and electrical conductivity may constitute an ideal for understanding the electrode-electrolyte interface, many more interesting phenomena can be observed through the use of materials where the carrier concentration can be manipulated through external or internal electric fields. Semiconductors offer a prime example of such tunability. Generally, semiconductor materials (see Figure 29) are defined in terms of having an energy gap (between the filled/valence states and the empty/conduction states) for electronic conduction to occur, and are classified either as (i) intrinsic, or (ii) extrinsic, depending on the extent of doping. For solids, the valence and the conduction states form energy bands and the energy gap $\left(E_{g}\right)$ may vary, at room temperature (300 K), from $\sim 26 \mathrm{meV}\left(=k_{B} T\right)$ to as large as $14 \mathrm{eV}$ (for LiF[187]). The most commonly used semiconductors, such as $\mathrm{Si}, \mathrm{GaAs}$, or $\mathrm{ZnO}$ have $E_{g}$ values of $\sim 1.1 \mathrm{eV}, 1.4 \mathrm{eV}$, and $3.2 \mathrm{eV}$, respectively. In the intrinsic/undoped case, the number of electrons equals the number of holes 
and the Fermi level, which is defined as the position where the probability of electronic occupancy is 0.5 , is mid-gap between the valence band (VB) and the conduction band (CB). Alternately, extrinsic semiconductors are doped with elements designed to yield an excess of either the electrons or holes in the $\mathrm{CB}$ or the $\mathrm{VB}$, respectively. Extrinsic semiconductors are then comprised of majority and minority carriers (e.g., in an $n$ - $(p$-) type semiconductor, where the majority (/minority) carriers are electrons (/holes[19]). In such materials, there is a greater relative change in the chemical potential for a given change in the minority carrier concentration, which then can drive the chemical/electrochemical reactions. Such a situation is analogous to the currents in a $p$ - $n$ junction[159]. The aspect that either the electron or the hole dominates a particular electrochemical process is pertinent to understanding charge transfer in semiconductors. Electron controlled processes proceed through the conduction band while hole controlled processes are mediated through the valence band, e.g., as in electrolyte reduction or oxidation, respectively.

The tendency towards the dominance of a particular carrier density (i.e., either due to the electrons- $n$, or the holes $-p$ ) is exacerbated as the $E_{g}$ increases, with a concomitant decrease in the intrinsic carrier concentration, $n_{i}$. This can be seen from the law of mass action, where $n_{i}=\sqrt{n \cdot p}=N_{c} N_{v} \exp \left(-\frac{E_{g}}{2 k_{B} T}\right)$, and $N_{c}$ and $N_{v}$ refer to the conduction band and valence band density of states, respectively[188]. For example, in the case of $n$-type $\mathrm{ZnO}\left(E_{g} \sim 3.2 \mathrm{eV}\right)$, electronic conduction is dominant and electron-hole interactions mediated through the electrolyte are unlikely (leading to efficient charge transfer), as redox potentials of the order of $3.2 \mathrm{~V}$ would be necessary, e.g., due to the paucity of energy states in proximity to the valence band. The larger electron mobility could also result from the trapping of the holes (at the oxygen ions), 
under an anodic polarization, leading to oxygen evolution, i.e., through the successive oxidation of the $\mathrm{O}^{2-}$ ion to $\mathrm{O}^{-}$and subsequently $\mathrm{O}$ or through peroxide formation by the association of two $\mathrm{O}^{-}$ions as $(\mathrm{O}-\mathrm{O})^{2-}$. It is also interesting to consider that open-circuit conditions would result in a concomitant accumulation of electrons (due to charge conservation) at the surface, and a steady state could be reached only through cathodic reactions, which consume the electrons. Generally, conditions corresponding to anodic polarization accentuate the efficiency of the hole trapping process (resulting in oxygen evolution) while the converse process, related to cathodic polarization yields hydrogen gas at the electrodes. Such issues also lead to the issue of photoninduced decomposition, where holes (which are really broken bonds) accumulating at the surface weaken the rigidity of the crystal.

As the majority and the minority carriers populate different bands the interaction of the electrode with the electrolyte must also consider such multiple energy levels. However, when the number of carriers is sufficiently large, the energy level spacing decreases and the semiconductor characteristics may adequately be described using metal electrode-like attributes. Such a description is referred to as the degeneracy approximation and the Fermi level is within one of the $\mathrm{CB}$ or the VB. The electrode-electrolyte interface may again be treated as that corresponding to metallic/conducting electrodes for intrinsic semiconductors as well, albeit with very small values of the conductivity as all the carriers populate only band, i.e., the lower energy VB.

4.6.1

a semiconductor interface
Charge configuration at 
For a passive semiconductor interacting with an external current collector (i.e., a metal or an electrolyte - without any surface chemical reactions), there would still be expected to be an interfacial charge rearrangement or transfer due to the presence of the surface, defects on the surface, as well as the metal/electrolyte. One may understand such a thermodynamic state, through the schematic diagram of Figure 29, based on a typical n-type semiconductor (left side) metal (right side) interface indicating the relevant parameters that may need to be considered, for the former. While such an arrangement of energy level/band diagram is characteristic of the bulk, it could be pertinent to nanoscale materials as well, when quantum confinement causes energy level separation (Section 1.2). Figure 29 is drawn for the case where the work function of the semiconductor $\left(\phi_{\text {semi }}\right)$ is less than that of the contacting material $\left(\phi_{m}\right.$, which is usually referred to that of a metal), i.e., $\phi_{m}>\phi_{\text {semi. }}$. The equivalent energy for removal of an electron is termed the electron affinity $(\chi)$. A Fermi energy level $\left(E_{F}\right)$ is also defined considering the highest energy level of electron occupancy with a position relative to the $\mathrm{CB}$ dictated by the level of carrier doping. Electron passage from the semiconductor to the contact surface induces an upward energy band bending of the $\mathrm{CB}$ by $V_{B O}$ (due to the equivalent reduction of electrons in the material) and presents an energy barrier $\left(\Delta \phi_{B n} \square\right)$ for motion of the electrons to/fro the material. The barrier is further reduced by $\Delta \phi_{n} \square \square$ through the inducement of a positive image charge[189] in the material formed subsequent to electron transfer from the material to the electrode contact. The voltage drop across the consequent double-layer (see also Section 2.3.1) is indicated by $\Delta_{o}$.

Typically, the requirement for the constancy of the electrochemical potential[190] as well as the vacuum[191] energies in equilibrium, throughout a connected system, enables the construction of such diagrams which may be considered to be generally well representative of 
bulk materials systems as well as chains of atoms[192]- the latter being characteristic of nanoscale materials. Additionally, as Figure 29 represents electron transfer from the semiconductor to the electrode contact, the deficit of electrical carriers in the former are now reckoned as holes, and populate the top of the VB. Alternate conditions, say with $\phi_{m}<\phi_{\text {semi }}$ would yield electron carriers in the semiconductor (with compensating hole states in the contacts), which now populate the bottom of the CB. The band bending in the material would be of the opposite curvature- such diagrams both in equilibrium as well as with externally applied voltage are ubiquitous in solid-state electronics textbooks $[19,159,193]$, and are fundamental to the understanding of the semiconductor interface.

It is then pertinent to note that electrical current from (/to) the contact to (/from) the material can only occur through carriers surmounting the intrinsic barrier at the contact/material interface. These in turn need (i) classical perturbing forces[166] or fluctuations[194], i.e., temperature induced excitation of the carriers (e.g., thermionic emission of carriers from/into the contact), (ii) the additional influence of applied electrical fields, or (iii) decreased barrier width aiding the quantum mechanical tunneling of carriers through the barrier (often considered through field emission). The current then increases exponentially with increased temperature or applied electric field. Consequently, in addition to the height of the barrier, the width of the barrier (approximately the length over which the band bending occurs) is also relevant and may be termed as a carrier depletion width $(W)$ A relationship of the form: $W \sim \sqrt{\varepsilon_{s}\left(V_{B O}-\Delta \phi_{n}\right) / e N_{d}}$ with parameters indicated in Figure 29 and $N_{d}$ being the carrier concentration in the region, is very well known[19]. The $W$ is often used as a measure of the distance through which the electrical carriers have to traverse to reach the interface and is 
directly related to the resistance experienced by the carriers. Tunneling, for example, is aided through a narrow $W$, which is obtained through increasing the $N_{d}$, i.e., heavy doping $\left(>10^{20} / \mathrm{cm}^{3}\right)$ - approaching a "metallic" condition and is the most desirable for obtaining the lowest resistance Ohmic contacts. The surface of the semiconductor is composed of states (surface states) distinct from the bulk states, due to a different environment, e.g., in terms of number of chemical bonds or due to the presence of foreign atoms from adsorbates, etc. As the surface area to volume ratio increases as the size of the material decreases (as is the case for nanomaterials: Section 1.1) surface states assume an even greater importance. It is then typically found that the interactions between the surface and bulk energy levels results in the formation of hybrid states, in the spirit of the Debye-Huckel model[17], distinct from either levels. For example, in a semiconductormetal contact, the hybrid states (referred to as Tamm states) are found in the bandgap region (between the VB and the $\mathrm{CB}$ of Figure 29), at around one-third of the $E_{g}$ reckoned from the valence band edge[18]. The extent of state filling is considered through an energy $\phi_{o}$ measured from the VB edge, specifying the energy below which all the surface states are filled so as to ensure charge neutrality at the semiconductor surface. The remaining difference - between the Fermi level and $\phi_{o} \square$, represents the net surface state charge density $\left(Q_{S S}\right)$ which together with the charge in the depletion layer of the semiconductor $\left(Q_{S C}\right)$ forms the total charge present in the material. An equal and opposite charge, of magnitude $Q_{m}\left(=-\left[Q_{S S}+Q_{S C}\right]\right)$, would now be induced in the metal in a very small interfacial layer of thickness: $\delta$. The $Q_{m}$ stored in the $\delta$ as well as the relative dielectric permittivity $\left(\varepsilon_{i}\right)$ of the interfacial layer could be used to infer the potential drop $\left(\Delta_{o}\right)$ through the application of Gauss Law[159]. The consequent movement of electrons from the $E_{F}$ to such surface states, within the semiconductor, could be expected to reduce the transfer efficiency of electrons from the contact to the semiconductor and lead to the 
necessity of applying higher voltages necessitating an overpotential - see Section 4.3 and discussion related to Eqn. (36). The surface states may thus screen the electrode charge and may confer metallic characteristics to the semiconductor surfaces- this would result in a voltage drop across the Helmholtz double layer (Section 2.3.1) causing a relative shift in the CB and the VB edges.

The charge redistribution results in the formation of an electron depletion/space charge layer; (of thickness, $W$ ) in the semiconductor (with dopant concentration: $N_{d}$ and dielectric constant: $\mathcal{E}_{s} \square$ ), analogous to a dielectric layer in a capacitor, bounded by two "plates" constituted from the semiconductor bulk and the contact. The equivalent space charge capacitance $\left(C_{S C}\right)$, due to such a capacitor can be estimated from standard semiconductor theory[159] and was previously stated in the form of Eqn. 9 (in Section 2.3.2). The frequency dependence of the $C_{S C}$ is revealing of the details of both microscopic processes, such as dielectric relaxation as well as material dependent characteristics such as extraneous energy levels and roughness of the electrode surface (Section 4.8.4). For example, on illumination of the semiconductor-contact interface (see Section 4.7) the band bending in the semiconductor may be reduced to zero and provides a measure of the relative defect density.

Indeed, the relevance of such phenomena to the fabrication of the first solid-state transistor by Bardeen and Brattain is well known[195]. It was realized that if electrons are present in the surface states there would be a proximal positive space charge region in the bulk, and the consequent formation of the thin double layer and voltage drop across the same could be more critical than the work function differences. The possibility of pinning of the $E_{F}$ to the defect states, where successive carrier addition negligibly affects the $E_{F}$ - until all the defect states are 
saturated, would be pertinent and results in band-bending and concomitant enhanced energy barrier for carrier transfer from the contact to the material. Consequently, the charge injection energy barrier: $\Delta \phi_{B n} \square \square \square \square\left|E_{g^{-}} \phi_{o}\right|$. It may be surmised in almost all cases of practical importance, that there would exist such an unsymmetrical energy barrier (Schottky barrier) between the semiconductor and the metal/electrolyte. It has been observed, that for nanostructure length scales $<1 \mu \mathrm{m}$, that the electrical transport to the surface would be limited by such barriers. However, for greater lengths and in lower dimensionality, there may also be a stronger influence of the defects/impurities which may induce resonant backscattering and reduce carrier mobility[20]. A more detailed discussion on the varieties of possible defects will be considered later in Section 4.8.2.

\subsection{2}

Charge transfer through

\section{the Semiconductor-Electrolyte interface}

We will now consider a non-degenerate semiconductor (where the Fermi level lies in the band gap between the CB and the VB) - electrolyte interface. Application of positive (/negative) voltages to the semiconductor increases (/decreases) the number of electrons and pushes up (/down) the $E_{F}$. However, the positions of the CB and the VB would not be affected as the band edges interfacing with the electrolyte are pinned due to the formation of a double layer with a fixed amount of charge, i.e., it is assumed that a changing voltage does not affect the double layer charge, as would be expected for adsorbed ions in the Helmholtz layer (Section 2.3.1). 
However, if a sufficiently strong voltage bias is applied to the electrode this would no longer be true and the band edges would shift. We will first consider, the situation where most of the applied voltage on the electrode only influences the Fermi levels in the semiconductor and the electrolyte bulk.

The semiconductor-electrolyte interface in the presence of charge transfer is then distinguished, from the interfacial situation considered in the previous section, in that electron exchange can occur to/from the electrolyte through both the VB and the $\mathrm{CB}$ as indicated in Figure 30, considering an n-type semiconductor. In case (a), when the electrolyte redox potential, i.e., the $E_{F, \text { redox }}^{0}$ is closer to the $\mathrm{CB}$, equilibrium of the electrochemical potentials dictates an equivalent energy for the $E_{F}$ in the electrode. The electronic current from the electrode to the electrolyte (cathodic current) results in a depletion of electrons from the CB near the semiconductor surface (which results in an upwards band bending and a lowering of the $E_{F}$ in the solid). However, the electron injection (anodic current) from the electrolyte to the electrode is relatively more difficult due to the paucity of equivalent energy levels. In case (b), when the $E_{F, \text { redox }}^{0}$ is closer to the VB a greater transfer of electrons results from the VB and a larger carrier depletion layer in the semiconductor. The loss of electrons at the surface, in either case, may be interpreted as an accumulation of holes. It can also be understood, from the difficulty of achieving equal amounts of cathodic and the anodic currents, as to why the exchange current densities (Sections 3.1.2 and 4.3 as well as the text related to Eqn. (36)) are smaller in semiconductors. Such smaller currents, on the other hand, may offer advantages in terms of enhanced sensitivities. 
While the above discussion has mainly focused on n-type semiconductors, the charge transfer occurs via holes in p-type semiconductors and contributes to the easier dissolution of these materials in comparison to n-type semiconductors. Anodic dissolution involving the application of negative bias on the electrode promotes hole accumulation at the semiconductor surface, breaking surface bonds and promoting such breakdown. In comparison, the difficulty of generating holes in n-type semiconductors, except through relatively high electrode biases that induces hole formation through electron tunneling from the VB to the $\mathrm{CB}$, contributes to their relative stability. Related to a further understanding of the details of electron transfer, say from an n-type semiconductor to an electrolyte redox level, a simple mechanism could involve the following two steps[196]: (1) donation of an electron from the semiconductor to a surface state, $S S$, through the process: $S S^{0}+e^{-} \rightarrow S S^{-}$, where the electron is temporarily localized, and subsequently (2) the oxidation of $S S^{-}$through interaction with the oxidizer levels $(O x)$ in the electrolyte through: $S S^{-}+O x \rightarrow S S^{0}+\operatorname{Red}$. The electron injection/capture may alternately be viewed as hole capture/emission, with analogy to the well known Shockley-Read-Hall (SRH) processes[159]. Such processes are electrochemically considered through cathodic or anodic polarizations and currents respectively. Whether electron or hole movement occurs is determined by the respective overlaps of the redox level-conduction band vis-à-vis that of the redox levelvalence band energy. Generally, the fluctuations in the solvent (considered through a solvent reorganization energy [197]) have an effect of broadening the energy levels and promoting charge transfer through surface states. While the exact representation of such processes has been subject to much debate, the inherent phenomenology can be appreciated through the introduction of an inductive element as well. The motivation could arise from several sources, i.e., the interaction of the surface states with the conduction and the valence bands and the current loops 
that could be formed through such processes. Additionally, charge localization would also imply a redistribution of the electrical potential over the surface and the occurrence of localized electrical fields.

The injection of minority carriers (i.e., holes for an n-type semiconductor) was postulated to add to a recombination impedance, corresponding to the interaction of injected holes with the majority carriers- which was manifested as output radiation, as in the case of the cathodic reduction of $\mathrm{Ce}^{4+}$ ions at $n$-GaAs electrodes. Viewed in terms of the formation of surface intermediates, the resistance and the capacitance would be related to the potential distribution and the rate constants of electron and hole capture. In some cases, both electrons and holes may both be involved, e.g., the well known anodic dissolution of p-type Si in aqueous HF solutions. One particular mechanism involves the successive (a) trapping of a hole in a surface bond, to form $\mathrm{Si}^{+}$, (b) thermal excitation of an electron from $\mathrm{Si}^{+}$to the conduction band yielding $\mathrm{Si}^{2+}$, and (c) the chemical oxidation of $\mathrm{Si}^{2+}$, by HF, to give $\mathrm{Si}^{4+}$, which corresponds to the Si dissolution.

The modeling of electron transfer through surface states, by way of equivalent circuit models, admits to extensive study of the dynamics of charge transfer through impedance spectroscopy[198] - see Section 3.3, as well as Raman spectroscopy[199]. For example, Raman peak shift data may indicate that that peak positions were increased in energy (a blue shift) under anodic potentials[91]. Such a shift was sought to be rationalized through a "contraction and stiffening" of the bonds, presumably due to electron extraction/hole doping. For metallic materials as well as metallic nanostructures, such as multiwalled nanotubes, graphene, etc., an energy shift to a larger value can also be observed for both electron and hole doping[200]. It should be generally noted that the peak shifts, in Raman spectroscopy, are a function of both the 
electronic energies as well as the lattice vibrations/phonons. Accompanied by loss /gain of electrons, the bonds become more (/less) stiff with a concomitant blue (/red) shift, indicating phonons of higher (/lower) energy. Typically, an adiabatic Born-Oppenheimer (ABO) approximation is employed to understand such electron-phonon interactions, and has been extensively used when electronic energy level separations are much larger than the phonon energies. Here, electrons adiabatically relax to their ground state configuration aided by phonons of lower energies and faster relaxation times. However, in metallic structures the band gap energies are comparable to the phonon excitation energies, and would lead to non-adiabaticity and deviations from the $\mathrm{ABO}[24]$.

The modulation of charge transfer through the use of semiconductor materials may be further exemplified through considering the excitation of charge carriers, e.g., through external illumination. The energy bandgap $\left(E_{g}\right)$ of the semiconductor is a convenient reference for perturbing carriers in semiconductors - only those photons (with energy $>E_{g}$ ) capable of exciting electrons from the valence baby to the conduction band can cause an effective carrier density variation.

\section{induced by photons: photoelectrochemical cells}

A practical application of charge transfer for energy transduction at the semiconductor-electrolyte interface is induced through light and is manifested through photoelectrochemical cells. Generally, the electrochemical response of a material (i.e., metal or semiconductor) to illumination, could be interpreted both in terms the charge 
re-distribution as well as the change in the reaction kinetics[201]. The (i) massive, intensity dependent, change in the minority carrier (electrons for p-type semiconductors and holes for n-type semiconductors) concentration on illumination, as well as (ii) the electron-hole recombination processes in semiconductors distinguishes their response from that of metals. As the carrier mobility (and the diffusion coefficients) of the majority and minority carriers are different, it is plausible that a potential difference be set up due to the differential migration - the Dember effect. The Dember potential ensures, for example, that an equilibrium electrical current passing through a specific region, e.g., the space charge layer is constant. However, when the relative change in the minority carrier concentration is smaller than the majority carrier concentration, such effects can be ignored. The coupling of electromagnetic waves with a redox system [201] could be used for generating electrical power through the photoelectrochemical processes, and is of relevance in creating new types of photovoltaic cells[202]. . The key driver is again the difference in the electrochemical potential between a solid electrode (as defined, through the $E_{F}$ ) and the electrolyte (the cell redox potential: $E_{\text {redox }}$; see Figure 24) through the individual oxidation and the reduction potentials. It should be noted again that the oxidation (/reduction) may be taken as the loss (/gain) of electrons (/holes) or the gain (/loss) of holes, by the electrolyte. The relative energy levels of the material (semiconductor) and the electrolyte may further be understood through reference to Figure 24 and Figure 30. With reference to a n-type semiconductor, Figure 31(a) from [203], indicates the excitation of electron-hole pairs by external illumination of energy greater than that of the bandgap. The difference in the electrochemical potentials of the electrons and the holes with respect to the 
electrolyte, may contribute to individual redox reactions in the electrolyte. The consequent electron/hole state energy difference is then responsible for the carrier transfer from a higher energy level to a lower energy level, which is equivalent to an alternative redox process and the generation of a voltage. This figure indicates that while the electrons are put out to the external circuit, the holes react with the electrolyte and the electrons coming in from the external circuit compensate the resident excess positive charges in the electrolyte. Alternately, Figure 31 (b) indicates one practical application of the above process to the electrolysis of water, creating $\mathrm{O}_{2}$ and $\mathrm{H}_{2}$ gas through oxidation and reduction of aqueous electrolytes.

The basic principle, with relevance to charge transfer, is then that photon illumination also causes (i) the photo-excited minority (/majority) carriers move to the surface (/ bulk), and (ii) that the differential charge separation causes the formation of a space charge layer and a space charge capacitance: $C_{S C}$ (see Section 2.3.2). The width of the space charge layer is then correspondingly reduced, whenever there is charge (electrons/holes) introduced in the layer, yielding an enhanced capacitance, with a larger increase due to the minority carriers. Consequently, a change in the carrier concentration $(\Delta N)$ relative to the original carrier concentration $\left(N_{o}\right)$, facilitated through photon illumination enhances the layer capacitance, $C_{s c}$ to a larger value of $C_{s c, \text { ill }}$ through the relation[201]:

$$
\frac{C_{S C, \text { ill }}}{C_{S C, o}}=\sqrt{1+\frac{\Delta N}{N_{o}}}
$$


Indeed, intensity modulated photocurrent spectroscopy (IMPS) may be used to yield quantitatively similar information to electrochemical impedance spectroscopy (see Section 3.3) and measure the relevant capacitances[204]. With respect to practical EC operation, photon induced processes should be considered in concert with the time scales for electrostatic discharge of the double layer (see Section 3.3 and Figure 17) on the electrode, i.e., the time for the latter should ideally be smaller than that required for steady state conditions in the space charge layer to be reached. Additionally, since the photo-induced voltage is of opposite sign relative to the intrinsic band bending, the electrode potential of a semiconductor with an anodic (/cathodic) process is shifted oppositely in the cathodic (/anodic) direction, at a given current, which may impact charge capacity. In addition to the reduction of the obtainable voltage, multiple or competing processes, such as hydrogen discharge at the anode could complicate the analyses. A further detailed discussion of photon excited charge transfer in nanostructured ensembles is given in Section 4.10, where we consider chemical capacitance issues related to a dye-sensitized solar cell.

\section{Specific}

\section{characteristics of nanostructured electrodes}

A major imperative for continuing research in charge storage is the exploration of new storage mechanisms as well as alternate materials systems where such mechanisms could be harnessed. We have seen, for example, that carbon based materials are ubiquitous, i.e., as graphite based anodes in battery systems - Section 2.2.2, and as activated carbon electrodes in ECs - Section 2.3. The advantages of an intrinsic layered nanostructure in the former and large surface area in the latter are the relevant nanoscale features. In this section, we aim to explore the 
features of nanocarbon constituted electrodes with respect to their constitution and non-idealities. Subsequently, the manifestation of nanoscale features on a larger scale, with respect to the inducement of roughness and wetting of the electrodes with the electrolyte will be considered.

\subsection{1}

\section{Nanostructured carbons}

For a good understanding of whether nanostructures are more efficacious with respect to charge storage, much care has to be taken to understand the material specific characteristics. For instance, it is interesting to ponder why the surface of a single layer graphene (SLG) sheet would have a comparatively higher capacity or energy density[205-207], as frequently posited. Indeed, earlier work [208][75] reported the $C_{d l}$ of a clean graphite surface as $\sim 20 \mu \mathrm{F} / \mathrm{cm}^{2}$, typical to any other thin film (Section 2.3.1) providing a benchmark for what may be experimentally observed. Additionally, multiple graphene sheets/few layer graphenes (FLGs), which may be considered to have a limiting case of bulk structures, have been shown to have relatively higher capacitance [209,210]. Consideration of intermolecular forces between the atomic-scale sheets [211] may need to be considered for charge storage. A fundamental basis for adsorption-induced enhancement of the capacitance may arise from van der Waals forces induced through the $\pi$ orbitals. Indeed, the larger currents at a given scan rate in FLG compared to the SLG samples may be accounted for through such considerations. Alternately, examining the pore structure of activated carbons (as discussed later in Section 4.9), the ratio of the pore wall thickness to the Debye length (Section 2.3.1) in the electrolyte would be relevant. Through comparison with a variety of activated carbons, it was deduced that enhanced capacitance, i.e., to a limit of up to $80 \mathrm{~F} / \mathrm{g}$ (see Section 2.4) may be obtained through a decreasing ratio only up to a limit. 
Physically, this would correspond to an overlapping of the screening lengths. These results may then be extrapolated to a single graphene sheet, where the potential is not constant and to realize the ability of graphene to function as a proper electrode, multiple layers, up to four, may be necessary[210]. Alternatively, it may also be concluded that multiwalled CNTs[76], where the electric potential is constant beyond a certain number of walls, with the added advantage of a large surface area and the ability to enhance the density of states over a larger area (Section 4.11) may be better electrodes compared to single layer graphene.

Useful nanoscale prototypes for understanding such influences are for example, the basal planes and the edge planes of graphite surfaces. It has been widely reported that the former are chemically inert, while the latter are considered to be sites of electrochemical reactivity[212215]. The in-plane $s p^{2}$ bonding on each constituent graphene sheet of the graphite involves the pendant $\pi$-groups, which may be indirectly involved in charge transfer through weak bonding and adsorption processes[216]. The enhancement in the surface density of states due to the formation of localized states[217] may also be correlated with an increased capacitance. While passivation of the surface states, say through a $\mathrm{C}-\mathrm{H}$ bond, may occur, it has been posited that the $\sigma \square$ bond would not interact with the $\pi$-orbitals and disrupt electronic structure. Additional energy states may be introduced through curvature-induced modification of single/multiple graphene sheets as would be involved in the formation of single-/multi-walled CNTs, respectively. For example, several varieties of graphite based on the HOPG (highly oriented pyrolytic graphite) motif, may broadly be classified into (i) the EPPG - edge plane pyrolytic graphite, and (ii) the BPPG- basal plane pyrolytic graphite, forms. The latter variety constitute a significant fraction of HOPG (typical distances between edge steps can be of the order of 1-10 $\mu \mathrm{m}[124]$ ) and are 
characterized by an average lateral grain size [212]- which may be correlated to the TuinstraKoenig correlation length $\left(L_{a}\right)$, - a parameter widely used in Raman spectroscopy. In other forms of carbon, such as glassy carbon (prepared through the high temperature and pressure treatment of polymeric resins), a larger extent of $s p^{2}$ bonding coupled with disorder yields CV characteristics intermediate to EPPG and BPPG. It was seen[124] from cyclic voltammetry, that in BPPG samples, (i) a large overpotential is needed for electrochemical reactions to occur as manifested through a larger $\Delta E_{p}$ between the anodic and the cathodic reactions (Section 3.1.1), and (ii) the current variation deviates from a linear diffusion based theory (Section 3.1.2), and indicative of the concurrent presence of closely spaced diffusion layers (as discussed later in Section 5.2 and with reference to Figure 53).

The extent to which the above studies indicate the relative absence of electrochemical reactions on basal planes, compared to edge planes, is difficult to gauge vis-à-vis the inevitable presence of atomic scale/nanoscale defects. It has also been found, through treating and simulating[212] each pristine/defect free BPPG constituted region surrounded by a EPPG, as an individual domain of diffusion that the magnitude of $\Delta E_{p}$ with a chemical reaction rate constant $\left(k_{\text {basal }}\right)$ is inversely proportional to the defect density. Correlation of the relevant diffusion domains in terms of the $L_{a}$ and the equivalent grain size would be appropriate, but does not seem to have been much considered in literature. While an upper limit value of $k_{\text {basal }}=10^{-9} \mathrm{~cm} / \mathrm{s}$ was used for modeling the basal plane electrokinetics (with $k_{\text {edge }}$ seven orders of magnitude larger at $0.02 \mathrm{~cm} \mathrm{~s}^{-1}$ ), these numbers are yet estimates. It was interesting to note that in an alternate analysis comparing the reduction of quinone on basal planes and edge planes[214], the electron transfer rate (now expressed in units of $\mathrm{s}^{-1}[218]$ ) of the rate constants of the latter were only 2-3 
orders of magnitude larger. It has also been observed, through scanning tunneling microscopy studies[219], that adsorption tracks the defect area (given that the proportion of the defect area to the total projected area has been estimated to be of the order of 0.01 to 0.1 ) only qualitatively but may be orders of magnitude higher than expected. There may also exist electrostatic interactions between the adsorbates and partial charges on the surface (also see Section 5.1), which may extend over a larger area than a charged defect. Moreover, functional groups as well as the polarity of the surface[220] may enhance the sticking coefficient of adsorbates at the monolayer level. It has also been speculated that adsorption could occur concurrently with intercalation (as with anodes in battery systems - Section 2.2.2), where the latter is more probable at the edge planes.

In summary, the electrochemical characteristics of nanostructured carbons in particular, and electrodes generally still remain to be both qualitatively and understood, with reference to the role of defects and disorder. Such an understanding is necessary in light of the wide usage of nanostructured electrodes either for reasons related to a large surface area/unit mass (Section 1.1) or the invoking of quantum mechanical phenomena (Section 1.2). The fundamental aspects of defects will be explored next.

4.8.2

Non-ideality: Charges at

\section{defects}

Analytical or computational research has almost always initially focused on modeling defect free nanomaterials (e.g., constituting the electrodes) to typically postulate impressive 
theoretical thermal, electrical and structural properties. However, deviations from ideality, e.g., through defects, considerably modulate practical electrochemical kinetics and charge transfer. The presence of defects, in any given structure, is entropically inevitable in that the addition of any impurity in any amount would necessarily increase the entropy: $S$ [221], in accordance with the third law of thermodynamics. Generally, the lower dimensional defects, e.g., point/zerodimensional defects, such as vacancies, interstitial or substitutional defects are more favorable compared to one- or two-dimensional defects such as dislocations or boundaries which impose a free energy penalty, due to the energy required for their formation $\left(E_{f}\right)$, to the overall structure. It can be derived[60] that the concentration of point defects $\left(n_{d}\right)$, relative to the total number of atoms in the sites in the structure $(N)$ is given by: $\frac{n_{d}}{N-n_{d}}=\exp \left(\frac{S}{k_{B}}\right) \exp \left(-\frac{E_{f}}{k_{B} T}\right)$.

However, defects do not necessarily hinder the performance of nanostructures, and in some applications, defects may even be necessary, e.g., defects in CNT chemical sensors act as binding locations for sensing functional groups as they are more energetic and subsequently more chemically reactive compared to pristine sites. Typically, functionalization is used to incorporate charged moieties such as - carboxyl, - amine, and -hydroxyl groups, to increase sensitivity and add specificity to sensing for the appropriate target molecules. Charged moieties could perhaps also achieve similar results. It would then be interesting to probe if functionalization is necessary to increase the electrocatalytic activity of a nanostructured electrode or if this can be achieved solely through charged defect introduction. We discuss then the influence of defects with respect to their natural and sometimes artificial appearance in model nanostructures such as one-dimensional carbon nanotubes and two-dimensional graphenes. 
Practically, such defect structures may be manifest in battery anodes (see Section 2.2.2) or carbon based electrochemical capacitors (Sections 2.3 and 4.9).

Much effort has been expended in calculating the formation energies of the defects though computing the difference in the energies between a defect free cell and a cell with a defect[222]. Such calculations are fraught with issues of correctly estimating the ground state energy and the aspect of obtaining the defect energy through subtracting two large numbers. While the accuracy of the individual methods and the involved approximations are beyond the scope of the present review, the interested reader is referred to relevant papers[222,223] and textbooks[224]. Aspects related to the formation energies and the energetics have also been discussed extensively for various materials in other texts[60,225], reviews[223,226-230], and associated papers [22,222,227,231-241]. Typically, the $E_{f}$ are greater than $1 \mathrm{eV}$ and may be as large as $14 \mathrm{eV}$ (for an adsorbed atom - single vacancy pair[223]). Many such defects are evident as a function of specific synthesis conditions and contribute to variable electrochemical characteristics. For example, a two-dimensional graphene sheet would be mainly comprised of basal plane constituted carbon, with edge sites at the periphery. A similar basal to edge site ratio may be extended to multi-layer graphene. However, in graphene synthesized through the microwave exfoliation of graphene oxide (MEGO)[113], the fraction of edge, as well as the hydrogen content, has been shown to be considerably small through electron paramagnetic resonance (EPR) based measurements and a $s p^{2}$ bonding character of $98 \%$ was ascribed through diffraction studies. Consequently, intrinsic or extrinsic defects in CNTs or graphenes can be defined at any location where the periodic hexagonal arrangement of $s p^{2}$ bonded carbon atoms (in a crystalline lattice) native to the constituent single-layer graphene sheet is perturbed. Intrinsic defects result from structural changes in the lattice without the introduction of impurity atoms, while the 
extrinsic defects may involve impurity atoms. In addition to structural effects, defects may also produce unique changes in the electronic properties. Moreover, impurity electronegativity and atomic or ionic size can affect the lattice interactions.

\subsubsection{1}

\section{Point Defects}

In the simplest case, when the lattice is missing an atom a vacancy may form. Additional lower dimensional defects such as vacancy clusters, interstitial adatoms, or lattice impurities are widely manifest in graphene-based nanostructures. Such defects induce changes in the bonding structure and can cause re-hybridization of the carbon atoms in addition to inducing local bond strain. The energy of formation for single vacancies, $E_{f, S V}$ is $\sim 5-9 \mathrm{eV}$. The variation in the formation energy of $\sim 4 \mathrm{eV}$, could be a function of the sample size, i.e., as in the diameter of a CNT[223] arising from differences in curvature. If a single vacancy is created, carbon atoms surrounding the vacancy are at a relatively higher energy state since they are not fully coordinated and have dangling bonds. Such higher energy carbon atoms may undergo JahnTeller distortions (geometrical distortion of non-linear molecules to reach a lower energy state[242]) to minimize the local energy, forming a pentagon and a nonagon with one dangling bond remaining, as seen in Figure 32. With respect to the graphitic motif, the presence of nonhexagonal rings has been considered to induce local Gaussian curvature, e.g., with pentagons leading to positive (spherical) and heptagons leading to negative (saddle like) curvature[223]. Consequently, it can be deduced through elementary electrostatics that this would increase the local charge density for any deviation from a planar configuration. 
Note that the Jahn-Teller effect in graphene and CNTs with low curvature, the $E_{f, S V}$ is $\sim 7$ $\mathrm{eV}$, which is relatively large. Vacancies can be mobile if enough energy is provided to overcome a geometry dependent migration barrier $E_{m b, S V}$ of $\sim 0.5-2 \mathrm{eV}[243]$. Subsequently, if two vacancies join, a double vacancy is formed with an energy of formation $E_{f, D V}$ of $\sim 4-5.5 \mathrm{eV}[243]$. In a double vacancy, there are no dangling bonds since two pentagons and a heptagon form which are relatively immobile with a migration barrier $E_{m b, D V}$ of $\sim 5 \mathrm{eV}$ [243]. Yet another type of defect, the Stone-Wales (S-W) defect, occurs when a C-C bond rotates and forms non-hexagonal rings while maintaining $s p^{2}$ hybridization and is unique to graphitic systems: Figure 33(a). The most energetically favorable rotation of the C-C bond was calculated to be $90^{\circ}$ (in Figure 33 (b)) with an activation energy $\left(E_{f, S W}\right)$ increase from $\sim 5 \mathrm{eV}$ to $\sim 10 \mathrm{eV}[222][244]$. The non-hexagonal pairings may cause bends or kinks along the CNT axis as externally manifested in Figure 33(c).

Extrinsic point defects may also arise from the addition of adatoms on the surface of the nanostructures. If the adatoms are smaller than the inter-graphitic spacing of $\sim 0.35 \mathrm{~nm}$, they can reside interstitially[245] facilitating the formation of two new covalent bonds (with a binding energy associated with the bond formation of $\sim 2 \mathrm{eV}$ [246]) to the surrounding carbons and an $s p^{3}$ character. In the case of carbon adatoms, the migration barrier is $0.4 \mathrm{eV}$, implying high adatom mobility along the graphitic surfaces. Generally, if a carbon adatom moves into a vacancy location, it may undergo rehybridization and covalently bond with the surrounding carbon atoms, e.g., if only a single vacancy is encountered, rehybridization would establish the symmetric hexagonal pairing typical of pristine graphene/CNT. Local bending can also occur if two carbon adatoms meet to form a dimer, which then interacts with the surrounding delocalized $s p^{2}$ network to form an inverse Stone-Wales defect, with a higher energy of formation $(\sim 7 \mathrm{eV})$, and less thermodynamically favorable compared to a S-W defect. 
For non-carbon adsorbed atoms or adsorbed particles, the electronic interaction energies would vary with the state and size of the adatom and its size (e.g., physisorption through van der Waals forces or chemisorption through bonding to the CNT surface). Covalently binding transition metals to the surface of CNTs using electrochemical techniques has been used[223,245,247] to identify the location of defects along the surface of a CNT, e.g., as shown in Figure 34. Given a typical metal reduction potential of $\sim 2.5 \mathrm{~V}$ (with respect to the standard hydrogen electrode), adatom bonding to defects would occur if the defect energies are larger. Additionally, covalent bonding may also occur between organic molecules and the graphitic surface/s guided through similar rationale[248].

When foreign atoms add as substitutional impurities into the graphitic lattice, say, for the purpose of doping, they may also be considered to be point defects. Thermodynamically stable substitutional impurities tend to be similar in atomic size and have a close number of valence electrons, e.g., boron or nitrogen. However, when coupled with vacancies, a broader variety of substitutions can occur. Transition metals may also form strong covalent bonds (with bonding energies $\sim 2-8 \mathrm{eV}$ ) at vacancy sites by coordinating with dangling bonds[223,249,250].

\subsubsection{2}

\section{One-dimensional defects}

A commonly observed defect in graphitic structures is an edge plane, where defects can induce different bond orderings and bond configurations. If bond reordering occurs without the addition of vacancies or other atoms, the armchair (Figure 35 (a)) and zigzag (Figure 35 (c)) configurations are formed. These configurations are the most stable of the four depicted in 
Figure 35, since they have the fewest dangling bonds, which represent centers of charge concentration. If vacancies are present on the edge plane, then variations of these configurations can occur as illustrated in Figure 35 (b) and (d).

The dangling bonds present at the edge plane can be passivated by various atoms or compounds giving rise to different bond strains and hybridization that each have varying effects on delocalized $\pi$-bonding and local geometry. An example is hydrogen passivation, where the hydrogen bonds to a lone pair and adds local $s p^{3}$ character. Many experiments have also been reported success in creating edge planes, e.g., through opening the endcaps of CNTs [251-253] through involving strong oxidizers, form carboxyl functional groups to which additional moieties can be attached. Such zero- and one-dimensional defects may also affect graphitic geometry by causing bending and varied edge plane stacking (two-dimensional defects). At a critical concentration of defects, the macroscopic graphitic structure will be unique and define the morphology. Figure 36 illustrates some of the different CNT morphologies, which are typically considered defect-induced structures. These include multiwalled CNTs, bamboo CNTs (with successive multiple walls stacked in a cup-/cone-like configurations), herringbone CNTs (where the graphitic planes are at an angle to the main axis of the CNT), or even multiple layer graphene sheets are some of the many manifestations. By way of other examples, in the Y-junction CNT shown in Figure 36(b) and the coiled CNT in in Figure 36(c), the defect carbons maintain strained $s p^{2}$ hybridization through Stone-Wales type rotations. Consequently, complementary pentagon and heptagon rings are formed and in turn induce a local contraction in the tube radius at the center of the defect forming a Y-junction. If the charge defect density is more extensive, complementary pairing of pentagon-hexagon rings can lead to a twisting or coiling of the CNT, 
where coil radius and the pitch may be dependent on the defect density induced through appropriate synthesis conditions[254,255].

4.8.3

Influence of defects on

\section{bandstructure and charge capacity}

Extrinsic defects and associated charges may also be purposefully induced in graphitic structures through ad-atoms/-particles, vacancies, substitutional impurities, etc. The methods for their introduction may, for example, be through: (1) irradiation with electrons or ions[232,237,259], and (2) chemical treatments, e.g., through oxidation induced wet chemistry. Generally, the methodology through which defects are introduced and the type of atoms or ions involved play a crucial role in how the defects interact with the electronic structure of the host lattice. For instance, the electronegativity and ionization potential of an adatom can determine, to a first-order, whether the adatom has a propensity for drawing electrons away or donating electrons to the surrounding bonds. The injection or withdrawal of electrons into $\pi$ - bonds may be termed doping. Since $\pi$ - bond electrons are extensively delocalized in graphene and CNTs, the $\pi \square$ and $\pi^{*}$ - orbitals may constitute the valence and conduction bands, respectively.

When electron deficient moieties are added to graphitic structures either through bonding, adatom introduction, or substitutional impurity addition, energy levels in proximity to the regular bands may be created. For example, when B or N (of approximately the same size as carbon) with one less/more electron than carbon is added to graphitic structure, the carbon atoms are replaced as shown in Figure 37(b) and (c). The charge density shifts creating a partial 
positive/negative charge on the surrounding carbons and new defect energy levels are created near the valence/conduction band, as shown in Figure 38. Instances of electron withdrawal and donation with respect to the graphitic structures may then occur when $\mathrm{B}$ and $\mathrm{N}$ are added substitutionally. Such downward or upward variations in the Fermi energy modulate the voltage and the consequent current in charge transfer from a given nanostructured electrode to the electrolyte (as discussed previously in Sections 4.3 and 4.6.2).

Adatoms/adparticles can also cause shifts in the electronic DOS: Figure 38, where due to the higher reactivity of defect sites, they could be trapped at the sites. Depending on the electronegativity of the adatom or the dipole moment of the adparticle, electron density can be shifted to or away from the $\pi \square$ bonds in graphitic structures. An example of electrostatic doping arises when exposing CNTs to oxygen, which adds on as an adparticle on the CNT. Since the oxygen electronegativity (3.44) is greater than that of carbon (2.55) on the Pauling scale, the introduction of oxygen shifts electron density away from the CNT, creating local positive charge. Inducing local charged regions along the CNT surface through defect manipulation and doping can be used to inherently change the bonding in CNTs through a variation in the Fermi energy. Changes in the net electrochemical potential would then dictate the reactivity of the constituent bonds and can increase electrocatalytic activity or charge transfer from the electrode (see Sections 4.3 and 4.6.2). 
Integral to evaluating the performance of nanostructured electrodes is whether the electrolyte is fully in contact with the constituent area, for charge transfer interactions to occur. Such issues may be discussed with respect to whether the electrolyte wets the electrode and is a function of the respective surface energies of as well as physical characteristics such as the roughness of the electrode, etc. Classical monographs [260] describe such interactions fundamentally by considering a wetting/spreading parameter $(S p)$ of the form: $S p=\gamma_{\mathrm{s}-\mathrm{a}}-\left(\gamma_{\mathrm{se}}+\right.$ $\left.\gamma_{\mathrm{e}-\mathrm{a}}\right)$, where the subscripts refer to the relative surface energies of the solid electrode-air, solid electrode-electrolyte, and the electrolyte-air interfaces, respectively. Generally, when $S p>0$, the electrode prefers to be wetted by the electrolyte and good contact would be ensured. It was postulated that idealized interactions between the individual constituents could make it possible to relate the $S p$ to the individual polarizabilities (surface energies being related to the square of the polarizability[211]) . Consequently, an electrolyte would spread on/wet completely the electrode, if it is less polarizable than the solid electrode. Concomitantly, it can be adduced that enhancing the polarization of the electrode, e.g., as in metallic materials or through the edge planes in graphite, or defects (see Section 4.8.2.2) would favor greater contact between the electrode and electrolyte. Such fundamental criteria may also be used for the design as well as intuitively understanding the relative performance of conducting polymer electrodes (see Section 6.2) where enhanced polarizabilities at the nanoscale may be obtained through (i) larger sizes of atoms/molecules or attached electrophilic/nucleophilic substituents[261] with large dipole moments, (ii) unsaturated bonds, or (iii) at lower temperatures.

The relative dielectric constants $(\varepsilon)$ of the electrolyte $\left(\varepsilon_{\mathrm{e}}\right)$ and the material constituting the electrode $\left(\varepsilon_{\mathrm{el}}\right)$ should also be considered, especially when porous media (say, in the form of 
activated carbon; also see Section 4.9) are used as electrodes. While van der Waals attractive forces are often assumed initially, the mutual forces may turn repulsive (and hence reduce electrode wetting) if the $\varepsilon_{\mathrm{e}}<\varepsilon_{\mathrm{el}}-$ the electrostatic energy (proportional to $\varepsilon \xi^{2}[189]-$ see Eqn. (1)) would be reduced if the electrolyte was not interacting with the electrode. For aqueous electrolytes, one should also consider the relative hydrophobic and hydrophilic character of the surfaces, e.g., the latter may be enhanced through the presence of polar moieties. On the other hand, hydrophobic surfaces may be stochastically wetted/de-wetted by water for pore sizes of the order of $0.7-0.8 \mathrm{~nm}[262]$ - see Section 4.8.1.

The scale of roughness may also determine the efficiency of charge storage, i.e., in addition to its influence on wetting another obvious aspect would be related to the actual surface area available for charge accumulation and/or charge transfer. A ratio of the actual area to the apparent projected area that has been used to describe the wetting characteristics, and may also be extrapolated to estimate the enhanced charge density of a rough surface (also see Section 4.9). Alternatively, another quantitative methodology involves the use of the current-potential curves obtained from surface adsorption based pseudocapacitance (see Section 5.1) [263], e.g., the ratio of the theoretical maximum capacitance to the measured capacitance would yield a measure of the roughness to within $\pm 40-50 \%[263]$. A roughness factor has also been used in estimating the capacitance of nanostructured electrodes, e.g., in dye-sensitized solar cells (DSSCs) - see Section 4.10. Here it was assumed that as the Helmholtz capacitance $\left(C_{H}\right.$ - see Section 2.3.1) would be similar for any two surfaces, and the roughness factor is used to multiply the capacitance of a smooth surface to obtain the capacitance of the rough surface[264]. 
Additionally, corrugations and the local curvature of the surface could simulate pore like behavior and influence the observed characteristics and be modeled per the discussion in Section 3.3.1 and discussion as related to Figure 20. Given the complexity of the problem and the general irreproducibility across samples, the tendency naturally has been to ignore such complications. In summary, monitoring the roughness of a real surface is a very difficult problem, akin to the problem of "How long is the coast of Britain?"- the answer would depend on the length scale used[265]. A prototypical example of roughness at the nano-, meso-, and macro-scale is practically manifested is through porous carbon based electrodes used in ECs (Section 2.3) and will be discussed next.

\section{Electrodes: A metric for high capacitance density}

While the overall charge capacity would generally be proportional to the volume, the double layer capacitance (Section 2.3.1) is a surface oriented process and is directly related to the available area over which the charge may be stored. The latter enables a quick charge and discharge, as only the surface is involved and accounts for the large power density as well as the low energy density. Consequently, porous carbon synthesized through pyrolysis of organic compounds, with a large effective area of $\sim 1200 \mathrm{~m}^{2} / \mathrm{g}[115]$, has been extensively used for electrochemical capacitors (ECs). Per an IUPAC (International Union of Pure and Applied Chemistry) report from 1982[266], the pores formed have been classified as either (1) micropores, with pore widths $<2 \mathrm{~nm}$, (2) macropores, with pore widths $>50 \mathrm{~nm}$, and (3) mesopores, with intermediate pore widths. The report also considers the type of physisorption 
isotherms (relating the amount of gas adsorbed to the relative applied pressure) typical to each pore classification. The experimentally observed isotherms are then deconvoluted, through numerical methods, to yield the pore size distributions[118]. The presence of primary or secondary porosity (i.e., porous pores) can also be indicated through electrochemical impedance measurements (see Section 3.3.1 and discussion as related to Figure 20). The specific pore structure would be responsible for both the capacity as well as the kinetics of charging and discharge.

Typical pore sizes in commercial activated carbons, used for ECs, are in the range of 0.5 $\mathrm{nm}$ to $10 \mathrm{~nm}[75]$. As the contribution of the macropores has been considered negligible $(<2$ $\mathrm{m}^{2} / \mathrm{g}$ ) to the overall adsorption[75], the focus has been to understand and optimize the micro- and meso-pore distribution in a given material. The adsorption in the latter, for example, involves both mono- and multilayer adsorption as well as capillary condensation, and the hysteresis in the adsorbed amount as a function of the applied pressure makes it difficult to understand the processes in detail. On the other hand, analyzing the effects of micropores requires the consideration of atomistic level interactions between the adsorbing species and the substrate. It was also thought that while structural influences, such as binding functional groups and roughness could be reduced in the micropores, electrical space charge effects, say due to the overlapping of the double layers, could be more significant. It seems possible to develop suitable synthesis or activation procedures to achieve desired pore distributions, e.g., to drastically reduce the formation of mesopores[127] or to achieve a specific tailored size distribution[267]. 
The aspect of slit-like pore morphology, used in many analytical and computational studies, was inferred from the observations that, for a given volume, flat molecules, e.g., benzene, seemed to be more easily adsorbed compared to globular moieties. Such molecular sieve-like characteristics, which manifest through both thermodynamic and kinetic perspectives[127], were further understood through calculations which predicted a lower potential energy for adsorption in slit like pores compared to adsorption on flat surfaces[268]. It was then inferred that for porous carbons, say derived from polymers, that the pores could be described, given the precision of the gas adsorption data, either through (i) a slit-pore like model with slit widths in the range of $0.43 \mathrm{~nm}-0.57 \mathrm{~nm}$ (with an average at $\sim 0.53 \mathrm{~nm}$ ), or (ii) a cylindrical pore model with pore diameters between $0.78 \mathrm{~nm}$ to $0.86 \mathrm{~nm}$. The underlying assumptions include a Lennard-Jones type interaction between the harmonically oscillating adsorbate and the pore surface/underlying volume. Such optimized slit widths also consider the effective radius of the carbon atom at $\sim 0.17 \mathrm{~nm}$.

A good fit of the total measured capacitance $\left(C_{\text {meas }}\right)$ - also see Section 2.3.2, in porous carbons was obtained through the relation: $C_{\text {meas }}=C_{d l}^{\text {ext }} A_{\text {ext }}+C_{d l}^{\text {micro }} A_{\text {micro }}$, through partitioning the contributions from the external surface area $\left(A_{\text {ext }}\right)$ as well as the micropore surface area $\left(A_{\text {micro }}\right)$ and indicates differential adsorption characteristics. It was also interesting to note from this study[75] that the double layer contribution from the former $\left(C_{d l}^{e x t}\right)$ of $\sim 74 \mu \mathrm{F} / \mathrm{cm}^{2}$ was more than three times greater than $C_{d l}^{\text {micro }}$ at $\sim 20 \mu \mathrm{F} / \mathrm{cm}^{2}$ for activated carbon microbeads while for activated carbon fibers, the $C_{d l}^{\text {ext }}\left(\sim 8 \mu \mathrm{F} / \mathrm{cm}^{2}\right)$ was smaller compared to $C_{d l}^{\text {micro }}$ (at $\sim 15 \mu \mathrm{F} / \mathrm{cm}^{2}$ ). As it was remarked earlier that the effect of surface functional groups is relatively minor in micropores, the $C_{d l}^{\text {micro }}$ in the range of $15 \mu \mathrm{F} / \mathrm{cm}^{2}$ may be considered typical of a basal plane 
graphite layer. However, the $C_{\text {meas }}$ would yet involve series contributions from the space-charge (Section 2.3.2) and the quantum capacitance (Section 4.11) and be smaller.

4.9.1

Anomalous variation of

\section{capacitance}

In porous carbon based ECs, there would be expected to be a correspondence between the capacitance and the relative sizes of the pores with respect to the ion sizes. Generally, maximal adsorbate - pore wall area interaction could occur if the sizes were equal, i.e., while a large pore size would result in mutual adsorbate interactions at the expense of adsorbate-wall coupling, a decreasing pore size would imply a gradual overlapping of the depletion layers between adjacent pore walls and a consequent diminished capacitance. However, at smaller pore sizes it was thought, based on experimental observations, that the breakdown of the solvation shell around the ions[127] in an aqueous electrolyte would be promoted by large applied potentials/electric fields. Consequently, the ions squeeze into the pores implying a closer distance of separation between the bare ions and the pore walls. From a simplistic parallel plate capacitor model (e.g., the Helmholtz model of Section 2.3.1) a greater or unexpectedly large anomalous capacitance would be expected - anomalous in the sense that it was hitherto believed that solvated ions with respect to pores smaller than $1 \mathrm{~nm}$ would be ineffective for charge storage [269]. Indeed, an uptick in the capacitance/unit area was observed: see Figure 39, in studies based on carbide-derived carbons, synthesized through the high temperature chlorination of carbides where porous carbon (with specific surface area up to $2000 \mathrm{~m}^{2} / \mathrm{g}$, and pore volume up to $1 \mathrm{~cm}^{3} / \mathrm{g}$ ) remains after the metals have been removed through reaction with chlorine[267]. 
However, the capacitance increase should be weighed considering a reduction of the dielectric constant $\left(\mathcal{E}_{\mathrm{r}}\right)$ is expected in confined volumes - from the rationale of a limited electric displacement for a given electric field $(\xi)$. Pertinently, the reduction of the $\mathcal{E}_{r} \square$ to as low as 4.7 (in aqueous solutions, where the bulk value for water is $\sim 78$ ) for ion-electrode distances of the order of $0.1 \mathrm{~nm}$ and to 7.8 for $0.2 \mathrm{~nm}$ was noted a few decades ago[77]. Other equivalent ways of understanding the reduced permittivity incorporate the effects of electrostriction - which is related to the dipole moments of the ions in the pores and results in an equivalent increase in the density, as well as dielectric saturation - where the molecular dipoles would be effectively all aligned in a confined space due to the electric fields[262]. The enhanced $\xi$ could arise from an ionic concentration gradient and also from osmotic forces. In the latter case, by the contact value theorem[211], a pressure $(P)=k_{B} T\left[\rho_{s}\right],\left(T\right.$ is the temperature and $\left[\rho_{s}\right]$ is the charge density at the surface) is built which may exert an entropic force for the ions to squeeze into the pores. A depolarizing field would counter the $\xi$ forcing the ion into the pore. The sum total of such effects would be manifested through effective $\varepsilon$ values much smaller than the bulk values. For an estimated electric field of the order of 1 Volt/ $/ .1 \mathrm{~nm}$, the $\varepsilon$ was effectively evaluated to be of the order of 12-14 [262] in aqueous electrolytes. However, the underlying assumptions, where a macroscopic parameter such as the $\varepsilon$ is used, involve an unchanging/homogeneous $\xi$ over a range corresponding to an ion-pair correlation function, and the exact numerical value of $\varepsilon$ is difficult to estimate[270].

Consequently, given a permittivity decrease, for the capacitance to increase by a factor of three, as indicated in Figure 39, the spacing (assuming a parallel plate capacitor model) would have to decrease at least by a factor greater than 3 . As the bare cation/anion diameters, say TEA 
(tetra-ethyl ammonium) $/ \mathrm{BF}_{4}$ (and tetrafluoroborate) are of the order of $0.68 \mathrm{~nm} / 0.33 \mathrm{~nm}$ in diameter (see inset to Figure 39), with solvated ions of $10 \%$ larger diameter[211], it is difficult to see how even the removal of solvation shells could accomplish such a large capacitance enhancement. However, if electrostriction was involved, accompanied by an increased density of the corresponding ions in the pore, a larger number of ions contributing to the charge transfer could account for the capacity increase. Such an issue is easy to understand, for example, where in the case of a pore of volume $1 \mathrm{~nm}^{3}$, multiple TEA/BF 4 ions (of volumes $\sim 0.3 \mathrm{~nm}^{3} / 0.03 \mathrm{~nm}^{3}$, respectively) could be accommodated, yielding an increased radial density.

However, it was indicated[262] that the free energies of the ions in the pores would be comprised of contributions from both the lowered configurational entropy (due to the lower accessible area) as well as a reduced solvation free energy (since the hydration number would be reduced). The determined consequence was that a stronger tendency to solvation in smaller ions, (e.g., $\mathrm{Na}^{+} / \mathrm{Ca}^{2+}$ have a greater tendency to solvate compared to $\mathrm{K}^{+}$and hence migrate less in nanopores[271]) would imply a greater barrier for the solvation shell stripped ion to be confined. In summary, it is probable that multiple mechanisms may be operative in the observed increase in the capacitance - related to both the solvated shell removal[127] as well as phenomena associated with electrostriction[262]. The aspect of a minimum critical radius, related to the configurational entropy (which diverges as the area available to the ion in the pore tends to zero) would impose a lower limit on the pore size.

Porous networks may also be considered assemblages of nanoparticle-based networks with specific characteristics. While this section has considered their use in area based energy 
storage we will next discuss the relevant scientific principles and utility as a bulk charge to energy transduction medium.

\section{Chemical}

\section{capacitance in nanostructures: Dye sensitized solar cells}

As the electrochemical potential is considered to be due to the sum of the electrostatic potential (due to the potential of a particular charge) and the chemical potential (due to the additional energy from a certain number of charges), the former gives rise to an electrostatic capacitance, while the latter yields a chemical capacitance. The inter-conversion of electrostatic to chemical potential forms is of course possible, as occurs for example in the measurement of an external potential/voltage difference between macroscopic electrodes in a photoelectrochemical cell (Section 4.7), as a consequence of internal excitations/charging of the chemical capacitances. A subtle distinction between an electrostatic capacitance and chemical capacitance is then that in the former, the electric field change at a particular point is associated with a carrier density change at the point, while in the latter, the carrier density change is distributed and occurs over a representative volume element.

Consider a macroscopic capacitor constituted of nanoscopic units arranged in a mesoscale architecture, e.g., $\mathrm{TiO}_{2}$ nanoparticles on the electrode of a dye sensitized solar cell: DSSC[272]. The potential difference between the electrodes of the capacitor would be mostly concentrated on the nanoparticles due to their large number as well as the interfaces. The consideration of a particular nanostructured entity is done through the change of the number of charge states: $N_{i}$, (for the $i^{\text {th }}$ nanoparticle) corresponding to both the free electrons (of 
concentration, $n_{c}$ ) and localized/trapped electrons (of concentration, $n_{T}$ for a given change in the chemical potential, $\mu_{i}$. A chemical capacitance $\left(C_{c h e m}\right)$ specific to such mesoscopic architecture has been postulated,)[273] and defined through:

$$
C_{\text {chem }}=\frac{d q}{d V}=\frac{e \partial N_{i}}{\frac{1}{e} \partial \mu_{i}}=e^{2} \frac{\partial N_{i}}{\partial \mu_{i}}
$$

From the thermodynamic expression: $\mu_{i}=\mu_{i}^{o}+k_{B} T \ln \left(N_{i}\right)$ and the definition of $C_{c h e m}$, we may derive that $C_{c h e m}=e^{2} \frac{N_{i}}{k_{B} T}$. Similarly, for $n_{c}$ electrons in the CB of a semiconductor (see Section 4.6.1), with $n_{C}=N_{c} \exp \left[-\frac{\left(E_{c}-E_{F}\right)}{k_{B} T}\right]$ - where $N_{c}$ as the effective density of the conduction band states, and assuming that the Fermi energy $\left(E_{F}\right)$ is well below the energy level, $E_{C}$ of the bottom of the $\mathrm{CB}$, i.e., the Boltzmann approximation to the Fermi-Dirac distribution[188], the equivalent conduction band capacitance, $C_{c h e m}^{C B}=e^{2} \frac{n_{c}}{k_{B} T}$. As an applied voltage on the DSSC electrode (typically of transparent indium tin oxide, so as to allow illumination to pass through) would be expected to change the $E_{F}$, such a capacitance can be distinguished through the exponential dependence of the $n_{C}$ on the voltage. Additionally considering electrons in trap states (say, $n_{T}$ ), the net charge capacity: $C_{c h e m}^{T}=C_{c h e m}^{C B}+C_{c h e m}^{\text {trap }}$, where the latter can be estimated from $C_{\text {chem }}^{\text {trap }}=e^{2} \frac{\partial N_{T}(\mu)}{\partial \mu}$ when the trap number distribution, $N_{T}(\mu)$ is known. However, in most nanostructure configurations, the form of $N_{T}(\mu)$ is unknown or disputed, i.e., whether the surface states are localized or exponentially distributed as well as lack of knowledge of their precise location within the bandgap (see Section 4.6.1). 
More generally, any local process that involves $N$ carriers (e.g., due to photon excitation in a semiconductor, which may be thought as the conversion of photon energy to an electrochemical energy) may be associated with the charging (/discharging) of an equivalent $C_{\text {chem }}$ and be represented as an element in a distributed equivalent circuit (see Section 3.3.1). The sum of the voltages across each $C_{c h e m}$ element would constitute the net voltage drop that can be obtained from the ensemble. As the $C_{c h e m}$ is defined for one nanoparticle - from Eqn. (45), in an ensemble as well as at high carrier concentrations, several such $C_{\text {chem }}$ are in parallel, and the $C_{\text {chem }}$ should be considered in addition to the other electrostatic as well as quantum capacitances (as discussed later in Section 4.11) in the system. The analogy of the $C_{\text {chem }}$ to a diffusion capacitance has been noted in terms of the charge difference between adjacent nanoparticles[273]. The concept of chemical capacitance then provides a link to understand charge transfer between separable volume elements, due to electric field screening/local potential drops, as may occur in nanostructured ensembles. Different sizes or shapes of nanoparticles may be classified through specific values of $C_{\text {chem }}$.

As previously discussed, the DSSC can be considered to be a prototype in which such charge transfer mechanisms could be understood from the macroscale to the nanoscale. In addition to such devices being relatively cheap, reasonable solar energy conversion efficiencies of the order of $13 \%$ [274] have been achieved. The macroscopic details of the working of such cells have been considered[202,203,275] and the principles reviewed[276] extensively in literature. Briefly, with reference to Figure 40 the basic DSSC cell consists of a photosensitive dye, adjacent to $\mathrm{TiO}_{2}$ nanoparticles (for increased collection area) placed on a transparent electrode (cathode, e.g., Indium Tin Oxide: ITO) in addition to an electrolyte and a counter 
electrode (say, Pt). On photoexcitation of the dye, the created electrons are transferred to the conduction band of the $\mathrm{TiO}_{2}$ and then onto the ITO and the external circuit. The oxidized dye is then replenished of electrons from the electrolyte, which in turn receives electrons through the external circuit. A typical reduction mechanism operative in the electrolyte uses the $\mathrm{I}^{-} / \mathrm{I}_{3}{ }^{-}$couple and proceeds via the reaction: $\mathrm{I}_{3}{ }^{-}+2 e^{-} \leftrightarrow 3 \mathrm{I}^{-}$. The multitude and complexity of charge transfer may be modeled through a sequence of capacitors. For example, several charge transfer and charge separation mechanisms, each associated with a potential difference and hence capacitance are apparent, e.g., (1) electron excitation in the dye, (2) transfer of electrons to $\mathrm{TiO}_{2}$, (3) subsequent transfer to the ITO, (4) movement of charge carriers in the external circuit, (5) transfer from external circuit to the anode, (6) anode to electrolyte, and finally (7) electrolyte to photoexcited dye. Associated with charge transfer is also the possibility of auxiliary processes, e.g., electron screening at the electrode or particle interfaces may induce Helmholtz and GouyChapman related double layer mechanisms (Section 2.3.1), across which there would inevitably be a potential drop, and associated capacitance. Figure 41 (from Reference [276]) indicates the correspondence of the capacitance (/or resistance) as a function of the intrinsic electrode/ $\mathrm{TiO}_{2}$ potential as measured through EIS (see Section 3.3) to the interfaces at which the charge transfer occurs. Additional inhomogeneities incorporating non-uniform charge transfer (say, from the $\mathrm{TiO}_{2}$ to the electrode) as well as heterogeneous charge transfer (due to transport of electrons as well as ions) in a disordered geometry, local variation in electrolyte concentration, surface roughness as well as surface states, and coverage dependent recombination are factors that make accurate modeling in terms of a definitive electrical circuit elements quite difficult. The nature of charge transport through nanoparticle interfaces is also not well understood. While we have been focusing much on complete transfer, the movement of the accompanying hole/positive ion with 
different charge mobility may also have to be considered for photoelectrochemical cells[276]. Indeed, it was considered that the operating principle of a DSSC was based on "differential electrode kinetics"[277]. Many treatments then model charge transfer processes through the assignment of a capacitance (through the ratio of the relevant charge to the assumed potential drop) to each identified mechanism and determine (a) the magnitude of the capacitance as well as (b) whether the relevant capacitances are in series or parallel. It was found for example, that in the presence of injected electrons in the $\mathrm{TiO}_{2}$ electrode conduction band (which would also contribute to a CB capacitance[278]), that the capacitance due to surface states (Section 4.6.1) was smaller compared to the Helmholtz capacitance $\left(C_{H}\right)[264]$ - Section 2.3.1. A carrier lifetime that may be inversely dependent on the illumination intensity [278], together with the ratio of the lifetime to the transit time across the device, are other factors that need to be taken into account for modeling and understanding the time response of a particular nanostructured device and should be considered in concert with the overall $R$ - $C$ associated time constants. EIS based measurements could be used to gauge the time responses and their variations, in addition to helping probe the validity of a given circuit model.

While the above discussion mainly considers capacitive elements, there would also be required from the principles of detailed balance, opposing charge transfer processes, e.g., say, those within the excited dye, from the photoexcited dye to the anode instead of the cathode, etc. Such dissipation of charge is typically considered under the umbrella of recombination processes, and modeled through equivalent resistances. Typically, the rates of charge recombination are a function of their relative concentrations, e.g., when the ion concentration in the electrolyte is sufficiently small the regeneration of the excited dye, due to redox reactions in 
the former, is considerably slowed. The presence of electron accepting surface states pernicious in nanostructures, along with their location within the bandgap, can enhance or diminish the recombination rates as well. When such surface states couple with the energy levels in the bulk electrolyte, additional charge transfer and dissipation would occur.

An optimization of DSSC functioning would need to consider all such charge transfer processes/capacitances in addition to the resistances. Only then can an equivalent electrical circuit, offering insight into the overall efficiency of the charge transfer and energy conversion, be constructed and be useful. Given the complexity of the charge transfer processes, very simple lower-dimensional modeling, where the control volume is large enough such that an effective averaging is performed over the disorder and various nanoscale charge transfer mechanisms has been attempted to date. Continuum equations incorporating electroneutrality and macroscopic constitutive equations (for flux balance, etc.) have been employed. Consequently, a single carrier (e.g., electron) diffusion model has been invoked[277], for reasons of simplicity yielding a diode-like equation (where the net charge current is the sum of the photocurrent and the dark/recombination currents). However, it has been discussed that such an equation does not describe practical DSSC cells[276], as a simple superposition of the photo- and the dark currents does not normally hold and details of electron transfer as a function of the specific barriers needs to be considered.

While electron transfer kinetics can be resolved in the frequency domain through EIS, the physical rationale for such processes in terms of electron motion has been considered through, e.g., (a) a continuous time random walk (CTRW) model, within the bulk of the electrode or the nanoparticle, or through the (b) multiple trapping (MT) model - constituted from 
diffusive transport through extended states modulated by specific (e.g., surface state dependent) trapping events. For instance, in the case of transport through nanoporous $\mathrm{TiO}_{2}$, the DOS has been modeled through exponential band tailing with a mobility edge (see for example, Figure 38), corresponding to an energy above which excitations could occur. The electron diffusion coefficient $\left(D_{n}\right)$, as well as the electron lifetime, in the MT model is effectively modified to an extent that depends on the trapping and detrapping events and may be quantified, e.g., to $D_{n}=D_{o}\left[1 /\left(1+\partial n_{T} / \partial n_{c}\right)\right]$, where $D_{o}$ is the diffusion coefficient in a trap-free system and $\partial n_{T} / \partial n_{c}$ parameterizes the variation of the ratio of trapped electrons $\left(n_{T}\right)$ to the number of electrons in the conduction band $\left(n_{c}\right)$. The lifetime $\left(\tau_{n}\right)$, on the other hand, is inversely related to $\partial n_{T} / \partial n_{c}$ with the concomitant conclusion that the diffusion length $\sim \sqrt{D_{n} \tau_{n}}$ is relatively constant with increased incident photon intensity. While electron hopping is a possibility and must also be included, such models set the stage for a Fermi-energy dependent diffusion coefficient.

For example, when small particles at the nanometer scale are used, e.g., in molecular photovoltaics [275] where $20 \mathrm{~nm} \mathrm{TiO}_{2}$ particles were used in dye sensitized solar cells (DSSCs), it has been noted that a single electron may result in a carrier concentration of the order of $10^{17}$ $\mathrm{cm}^{-3}[202]$. However, such a concentration may not be transduced to an equivalent conductivity and resistance, as (a) the diffusion constant and carrier mobility may not be easy to deduce, and more importantly, (b) due to the presence of carrier traps. Additionally, the injected electronic charge may be screened by the surrounding cations in the electrolyte reducing carrier drift. This has the interesting consequence, with implications for reduced efficiency, that electron motion must be accompanied by cationic motion. Such electron transfer to the anode has been 
considered in terms of a transmission line model[144] - also see Section 3.3.1, where each particle is considered to be a resistor with pendant charge transfer resistance and capacitance to the surrounding electrolyte.

Additionally, there are issues with respect to the mechanisms as well as the efficiency of charge transfer from the sensitizer dye to the electrode, related to (a) the metal to ligand charge transfer, (b) the specific paths of electron transfer, with regard to the occurrence of percolation related phenomena. On excitation of the sensitizer, it was considered that the electron density shifts from the metal (typically, Ru) to the ligands which inject electrons into the semiconductor[202]. The ligands themselves are bound through carboxylate groups to the semiconductor surface. It was discussed that such a short binding group enables charge transfer to occur in time scales of the order of $10 \mathrm{fs}$ [279] with orders of magnitude increase in transfer time (to ps) with a change of length to even $1 \mathrm{~nm}$. While the nanostructure is mostly surface, related to the aspect of a large collection efficiency, there is a concomitant presence of a large number of surface states/defect levels which trap electrons and reduce carrier collection efficiency[278]. Since charge transfer in general is accompanied by/due to a potential difference the related bridging parameter is the capacitance - which appears in several guises. We will next consider the manifestation of the capacitance in a quantum-mechanical context, where confinement and discrete energy levels play a major role. 
The term quantum capacitance was initially invoked to model electron gases and transport in lower dimensional nanostructures[280]. The essential idea, e.g., was that a 2dimensional electron gas (2-DEG)[11,16], when placed between two metallic electrodes at different potentials would not be completely screen the intrinsic electric field between the electrodes. Consequently, and also due to the limited density of states (DOS), a finite capacitance - termed as a quantum capacitance $\left(C_{Q}\right)$ could be ascribed to the 2-DEG. The $C_{Q}$ would be in series with the electrostatic capacitance (say, $C_{d l}$ ) (see Section 2.3.1) between the macroscopic metal electrodes, implying that the smaller of $C_{Q}$ or $C_{d l}$ would determine the overall capacitance. Note that for perfectly metallic electrodes (Section 4.1) the $C_{Q}$ would be effectively infinite due to their ability to completely screen the electric field. Consequently, for the observation of a maximum capacitance, the $C_{Q}$ needs to be as large as possible.

Since $C_{Q}$ is essentially a property of incompletely filled/limited DOS, it would be quite important to consider in nanostructures and associated device modeling[281]. We model quantum capacitance $\left(C_{Q}\right)$, which is relevant with nanostructures such as graphene or CNTs, with a finite $\operatorname{DOS}(E)$, as depicted in Figure 42(a). The increase (/decrease) of the Fermi energy $\left(E_{F}\right)$ of the CNTs could be significant, relative to the bulk electrolyte, when charge carriers of a single type, e.g., electrons of magnitude $d Q(=e \cdot d N)$, are added (/removed) due to an applied voltage change $(\Delta V)[11]$.

An effective capacitance could therefore be defined for a given electrode, considering the DOS at the $E_{F}$, as follows: 


$$
C_{Q}=\frac{d Q}{d V}=\frac{e d N}{\frac{1}{e} d E_{F}}=e^{2} \operatorname{DOS}\left(E_{F}\right)
$$

While there seems to be a close correspondence of such a DOS based quantum capacitance to $C_{\text {chem }}$ - as in Eqn. (45), the latter is (a) local to a small volume element, i.e., due to electrostatic potential accommodation over a small volume element, and hence (b) better defined from a classical/thermodynamic point of view. We model the net device capacitance in Figure 43, as a series combination of $C_{d l}$ and $C_{Q}$.

Correlating such capacitance contributions from prototypical nanostructures, where electrochemical data is available, will now be discussed. In work, e.g., on graphene sheets, [282], [283], the $C_{Q}$ of a single graphene sheet has been derived analytically, with an aim of application towards MOSFET (metal oxide semiconductor field effect transistor)-like devices. It was experimentally seen that a higher $C_{Q}$ was obtained the smaller the number of graphene layers, i.e., SLG (single layer graphene) had a greater $C_{Q}$ compared to FLG (few layer graphene) [207]. Correlations between the adsorbed ions in the Helmholtz layer with the $\pi$ - electrons were also invoked as yet another mechanism to narrow the width of the double layer (Section 2.3.1). An enhancement, by $\sim-0.15 \mathrm{~V}$, of the point of zero charge (PZC), with increasing number of layers indicated a positive charging of the surface. However, as adsorption was ruled out, this may imply a positive surface doping due to space charge effects extending from the outer to the inner graphene layers in a FLG sample. While a reduced electrostatic screening was invoked, it is unclear what this implies in the context of the mutual interaction of the graphene layers in FLG. It may be thought that the DOS at the Dirac point would increase proportionally to the number of layers implying a larger $C_{Q}$ for the FLG. Typically, the carrier concentration $(N)$ studied was less 
than $2 \cdot 10^{12} / \mathrm{cm}^{2}$ with concomitant $C_{Q}$ values of the order of $10 \mu \mathrm{F} / \mathrm{cm}^{2}$ (see Section 2.3 .2 for a perspective on the magnitude of these values)[283]. Additional contributions from the induced charges (through an applied bias) from charged impurities due to "local potential fluctuations and electron/hole puddles" were also considered. While the comparison was then between the series addition of the gate oxide capacitance and the $C_{Q}$, it is then aimed to consider, as previously discussed, the series addition of the $C_{d l}$ with the $C_{Q}$, as appropriate for an electrochemical capacitor (EC). Multi-walled CNTs (with concentric nanotubes of gradually decreasing perimeters) are ideal for this purpose - see Figure 43, as they have the attributes of being metallic while avoiding band gap related space-charge capacitance (Section 4.6.1). The $C_{Q}$ variation was estimated from a more fundamental perspective through considering the gradual population of successive bands. Such population/carrier concentration increase would increase the density of states at the $E_{F}$ and enhance $C_{Q}$ as indicated through Eqn. (46).

We first seek to understand the $C_{Q}$ characteristics of MWCNT ensembles (with average individual MWCNT diameter of $20 \mathrm{~nm}$ and spacing $200 \mathrm{~nm}$ on $\mathrm{Si}$ substrates) as electrodes[169,172]. The DOS of a constituent wall in a MWCNT, following previous methodology[11], can be modeled as a rolled graphene sheet (infinite in the $y$-direction and both periodic and finite in the orthogonal $x$-direction). It was assumed that the walls are independent of each other[284], with the implication that the total DSS can be obtained as the sum of the DOS for each constituent wall. We considered zigzag (Section 4.8.1.2) CNTs (involving rolling of the graphene sheet in the $x$ - direction), as this category encompasses both semiconducting and metallic CNTs[285]. As we consider relatively large diameter CNTs, the details as to how graphene is rolled to yield CNTs, i.e., whether zigzag or armchair or chiral[286] - also see 
Section 4.8.1.2, will not influence the $C_{Q}$. The exact dispersion relation for a graphene sheet, through the tight-binding approximation[11,287] is:

$$
E\left(k_{x}, k_{y}\right)= \pm \gamma_{1} \sqrt{1+4 \cos \left(\frac{\sqrt{3} a k_{y}}{2}\right) \cos \left(\frac{a k_{x}}{2}\right)+4 \cos ^{2}\left(\frac{a k_{x}}{2}\right)}
$$

In Eqn. (47), $a=\sqrt{3} a_{0}$ where $a_{0}(=0.142 \mathrm{~nm})$ is the C- C bond length and the overlap integral $\gamma_{1}=2.9 \mathrm{eV}$ [284]. The $20 \mathrm{~nm}$ MWCNT with 15 walls can be approximately indexed through $[N, 0]$ (with $N=250$ for the outermost wall, and decreasing by 10 for each successive inner wall), and was effectively one dimensional since $k_{x, n}=\frac{2 q \pi}{N a}(q$ : sub-band index $)$, while $k_{y}$ is continuous. The DOS for a single sub-band is then $\frac{4}{2 \pi} \frac{d k_{y}}{d E}$ with $k_{x n}$ held constant, and the 4 in the numerator accounted for the electron spin degeneracy and the positive/negative $k_{y}$.[110]. Since $C_{Q}$ is a function of $E_{F}$ - Eqn. (46), we needed to estimate an appropriate value for $E_{F}$. In a graphene sheet with no impurities, each carbon atom provides one electron to the $p_{z}$ orbital, yielding semi-metallic behavior and implying ${ }^{19}$ an $E_{F}=0 \mathrm{eV}$, and zero carrier density $(N)$ at $T=0 \mathrm{~K}$. However, $N$ could range around $4.6 \cdot 10^{12} \mathrm{~cm}^{-2}$, corresponding to the twodimensional carrier density interpolated from the experimental value for bulk graphite[288] of $10^{19} \mathrm{~cm}^{-3}$, i.e., through $\left(10^{19}\right)^{2 / 3}$. With such variability in $N$, e.g., due to defects, [283], etc., attempting an exact $E_{F}$ would yield imprecise values, and it could then be appropriate to estimate the $N$ by approximating the CNTs as sheets of graphene and calculating the DOS, as was done here. The $N$ of $4.6 \cdot 10^{12} / \mathrm{cm}^{2}$ is then only posited as a representative number for the purpose of illustrating the concepts. The actual $N$ in any particular sample could either be below or above[289] this number with a corresponding decrease/increase in the $C_{Q}$. From the 
total carrier concentration at the Fermi energy, $n\left(E_{F}\right)=\int_{0}^{\infty} D(E) f(E) d E$. The $f_{F-D}(E)$ is the Fermi-Dirac function and was approximated as a step function in the calculations, as the difference between the value of $f_{F-D}(E)$ with a finite temperature $(T=300 \mathrm{~K})$ and with $T=0 \mathrm{~K}$ was at most $5 \%$. The $E_{F}$ values were found to range around $278 \mathrm{meV}$ (with $n=4.6 \cdot 10^{12} \mathrm{~cm}^{-}$ ${ }^{2}$ ). Computing $E_{F}\left(k_{x, n}, k_{y}\right)$ from Eqn. (47), and then $C_{Q}\left(k_{x, n}, k_{y}\right)$ from Eqn. (46), pairs of $E_{F}$ and $C_{Q}$ for all sub-bands $k_{x, n}$ over the Brillouin zone for $k_{y}$ are plotted in Figure $42(\mathrm{~b}) . C_{Q}\left(E_{F}\right)$ is constant initially due to the metallic CNTs, up to $\sim E_{F}=50 \mathrm{meV}$, due to the constituent metallic NTs with finite and constant DOS, where $C_{Q}$ does not increase as there is no sub-band contribution from the NTs. The staircase like structure in the variation results from the contribution of successive sub-bands to the DOS. At $E_{F}=278 \mathrm{meV}$ we estimate, in units of capacitance per NT length, $C_{Q}=48 \mathrm{fF} / \square \mathrm{m}$. The linearity in the plot justifies starting with the graphene $E_{F}-k$ relation to estimate the $E_{F}$ of the CNT from $N$. We next consider the two major components, which add in series, of the $C_{d l}$ : (i) a Helmholtz capacitance $\left(C_{H}\right)$ due to a Coulombic attraction, and (ii) a Gouy-Chapman $\left(C_{G C}\right)$ capacitance due to the diffusive distribution of ions in the electrolyte ${ }^{3}-$ Section 2.3.1. An area average $C_{H}$ can be computed from a spatial separation corresponding to the ionic radius ${ }^{11}$ (e.g., $r \sim 0.278 \mathrm{~nm}$ for $\mathrm{K}^{+}$ions in $\left.\mathrm{K}_{3} \mathrm{Fe}(\mathrm{CN})_{6}\right)$ and is equal to $\mathcal{E} \square r$. The $C_{G C}$ is estimated from the voltage drop $(\phi)$ across the diffusive region (which is of the order of the Debye length, $L_{D}$ and is equal to $(\varepsilon \square d) \cosh$ $\left(e \phi \square \square k_{B} T\right)$ - see Eqn. (8) in Section 2.3.1. Consequently, the series addition of the capacitances yields: 


$$
\frac{1}{C_{d l}}=\frac{r}{\varepsilon}+\frac{d}{\varepsilon} \frac{1}{\cosh \left(\frac{e \varphi}{2 k_{B} T}\right)}
$$

At smaller $\phi \square \square \rightarrow \square \square \square$ the $C_{d l} \rightarrow C_{G C}$, at $\square \phi \square \square \square \square \square \square k_{B} T$ ) the $C_{H}$ and $C_{G}$ are comparable, and at a larger $\left.\phi \square \square \square \square \square \square \square k_{B} T\right)$, the $C_{d l} \rightarrow C_{H}$. With a range of $\phi$ from zero to $278 \mathrm{mV}$ (corresponding to the $E_{F}$ ), we estimate from Eqn. (48), a range of $C_{d l}$ for an electrolyte concentration, $I$ (in moles $/ \mathrm{m}^{3}$ ), from $\sim 7.3 \sqrt{I} \mu \mathrm{F} / \mathrm{cm}^{2}$ to $\sim 255 \mu \mathrm{F} / \mathrm{cm}^{2}$. In order to compare to the one-dimensional quantum capacitance $C_{Q}$ estimated above, we convert the units of $C_{d l}$ by multiplying by $2 \pi r$, where $r=10 \mathrm{~nm}$ is the outer MWCNT wall radius. The corresponding range is then from $4.6 \sqrt{I} \mathrm{fF} / \mu \mathrm{m}$ to $160 \mathrm{fF} / \mu \mathrm{m}$. For a given $I$, say $3 \mathrm{mM}$ as in the experiments (see Table V of Ref. [172]), the $C_{d l}$ is calculated to be $7.9 \mathrm{fF} / \mu \mathrm{m}$. With $C_{Q}=48 \mathrm{fF} / \mu \mathrm{m}$, this results in a $C_{\text {tot }} \sim 6.8 \mathrm{fF} / \mu \mathrm{m}$. Generally, the electrostatic interaction between surfaces of different geometries decays with a characteristic decay length equal to the $L_{D}{ }^{21}$. Equivalent capacitances are then obtained for the planar/cylindrical cases.

The capacitance per projected electrode area is the product of the obtained $C_{t o t}$, the average CNT length, $L(=100 \mu \mathrm{m})$, the estimated CNT density on the substrate, of $\sim 2.5 \cdot 10^{9} \mathrm{~cm}^{-2}$, and the projected surface area of the electrode, $A_{\text {proj }}\left(\sim 0.25 \mathrm{~cm}^{2}\right)$ yielding an expected capacitance value per projected area of $\sim 1700 \mu \mathrm{F} / \mathrm{cm}^{2}$. Dividing this value by the weight of the CNTs $(\sim 40$ $\mu \mathrm{g}$ ), the capacitance values, in $\mathrm{F} / \mathrm{g}$, were computed and are shown in comparison to the experimental values (details have been previously reported[169,172]) in Figure 44, which then indicates the relative magnitudes of $C_{Q}$ relevant to the measured capacitances and implies that 
the $C_{Q}$ is more significant at lower electrolyte concentrations when the CNT is sufficiently isolated so that its DOS is small. It is generally observed from the figure that while higher electrolyte concentrations may be adequately modeled through the use of $C_{d l}$ alone, lower concentrations need $C_{Q}$ as well. As $I$ increases, charge transfer between CNT and electrolyte may be more likely, reducing isolation and increasing the CNT DOS effectively so that $C_{Q}$ increases and becomes insignificant, as per Eqn. (46). For example, the magnitudes of both $C_{Q}$ and $C_{d l}$ are comparable and suggest an explanation for the considerable (up to $300 \%$ ) increase in $C_{\text {meas }}$ when the CNT constituted electrodes are subject to argon plasma processing ${ }^{8,11}$. Such exposure was hypothesized to introduce charged acceptor like defects into the NT's carbon lattice, through argon abstracting electronic charge from the carbon bonds (Section 4.8.2). Much like surface states in semiconductors ${ }^{22}$, the fixed charges in the CNT lattice are immobile, and do not respond to applied voltage and would not contribute directly to the $C_{d l}$. However, the added charge density, e.g., through ion irradiation, affects the Fermi energy and enhances $C_{Q}$. A higher $C_{Q}$ closer to $C_{d l}$ enhances the maximum $C_{\text {meas }}$ that could be obtained from a given system. In summary, it can be concluded that the limits to the magnitude of the capacitance that can be obtained from CNT or nanostructure based electrochemical capacitors is a function of the series combination of both the $C_{d l}$ as well as the $C_{Q}$. In a situation where both are comparable, one would need to increase the $C_{Q}$, say through varying the charge density and maximize the total capacitance. Indeed, it was hypothesized[169,172], that ion irradiation ( e.g., through plasma processing) would introduce fixed charges, and that the $C_{Q}$ increases significantly.

An enhancement of the DOS of CNTs through nitrogen doping (up to 7.4 atom \%, as determined through XPS : x-ray photoelectron spectroscopy) and related disorder (due to edge 
planes as well as structural effects) was deduced through studies on free standing CNT electrode mats[290] - also see Section 4.8.2. The study considered a roughness factor (in the range of one - five, through the ratio of the area determined through chronocoulometry to the geometrically projected area- see Sections 4.8.4 and 5.2) to estimate the capacitance density. A shift in the PZC by $-0.6 \mathrm{~V}$ (indicating positive charges on the CNTs) was observed on $\mathrm{N}$ doping, in the capacitance vs. voltage profiles and attributed to pyridinium groups (through XPS analysis) at the edge plane sites - Section 4.8.1.2. A similar positive shift in the $E_{F}$ of $\sim 0.4 \mathrm{eV}$ was seen through the $\mathrm{N}$ incorporation, in UV photoelectron spectroscopy (UPS), which also indicated an increased work function, i.e., from $4.2 \mathrm{eV}$ for undoped CNTs to $4.5 \mathrm{eV}$ at 7.4 atom\% N. The net capacitance comprised of a series combination of the $C_{H}$ and a $C_{Q}$ was deconvoluted to yield the DOS values of the order of $10^{20}-10^{21} \mathrm{~cm}^{-3} \mathrm{eV}^{-1}$. The DOS seemed to increase with increasing $\mathrm{N}$ content and was inversely proportional to the Tuinstra-Koenig defect correlation length $\left(L_{a}\right)[291,292]$, perhaps indicating that the disorder was also responsible. Interestingly, it was found that Fe impurities, which could arise in CNT growth, did not contribute to the DOS. While the number density of carriers $(N)$, estimated through the relation: $N\left(E_{F}\right)=0.693 \operatorname{DOS}\left(E_{F}\right) k_{B} T$, was shown to increase with $\mathrm{N}$ atom content, the carrier mobility ( $\square$ ) as deduced from electrical conductivity measurements on the CNT mats showed an order of magnitude decrease possibly outweighing the $n$ increase, again indicative of the influence of disorder. The disruption of the $\pi$ - bond network and an increased $\sigma \square \square$ bonding due to the $\mathrm{N}$ akin to a conversion from an $s p^{2}$ to $s p^{3}$ bonding (as has been discussed in Section 4.8.2) was posited as the underlying mechanism.

\section{transfer processes}


Faradaic reactions, corresponding to charge transfer/redox interactions, may also infuse charge into the surface or the near surface regions of a material. The consequent charge storage may be harnessed to improve the energy capacity and the power density (due to the relatively quick release of the energy). The consideration of such mechanisms has been the topic of extensive investigations recently, due to the seeming possibilities of bringing battery-like energy densities to ECs. In this section, the focus will be on the fundamentals behind such charge storage, one manifestation of which is the pseudocapacitance characteristic.

\section{Pseudocapacitance}

An intermediate case of current-voltage behavior indicating a transition from a purely battery-like behavior to an capacitor has been termed pseudocapacitance (PC)[126,293] and refers to the attributes of those devices where while capacitor like behavior is observed (e.g., a linear decrease of voltage with removal of charge as well as a discharge current, $I=C s$, where $s$ is the voltage scan rate) - such behavior is strongly dependent on the chemical composition and constitution of the surface. The study of PC has been considered quite important recently[294], as it seems to represent an opportunity to harness additional electrical charge and energy from the surface through sustained Faradaic/redox reactions (in addition to the double layer charge) and has been presented as a possible way to bridge the energy density of batteries to that of traditional capacitors, as represented through Figure 45. The parallel addition of the PC to the conventional capacitances enhances the overall charge and energy capacity. Considering a charged surface with $Q=100 \mu \mathrm{C} / \mathrm{cm}^{2}$ [211] - corresponding to a single charge in an area of 0.16 $\mathrm{nm}^{2}$, or an average charge separation of $0.4 \mathrm{~nm}$, the maximum $C_{P C}$ would be of the order of 1 
$\mathrm{mF} / \mathrm{cm}^{2}$, which is orders of magnitude larger than typically encountered double layer capacitances.

The PC terminology itself is generally used to contrast such surface based adsorptive characteristics from the phenomena prevalent in conventional electrostatic capacitors with solidstate dielectrics or double layer capacitors formed due to electrodes immersed in liquid/fluid electrolytes. It is to be noted that in such conventional devices, the differences between the electrical/electronic characteristics vis-à-vis the surface composition of the devices is neglected. This is done as (i) many surface Faradaic reactions typically occur over a small range of potentials (of the order of $R T / F, \sim 26 \mathrm{mV}$ at room temperature -as discussed later with reference to Figure 49) and (ii) that the surface is only a small part of the whole in bulk-like materials. However, there are some notable exceptions to (i), as subsequently discussed with reference to Figure 46, and (ii) may not always be appropriate at the nanoscale. As there is always expected to be some adsorbate on the electrodes at any voltage, the practical importance of the PC arises from the aspect that it would act in parallel to the conventional $C_{d l}$, which of course, results in a greater overall capacitance and higher energy density (see Figure 21). PC is also characterized substantially up to monolayer deposition of adsorbates, and subsequent deposition is classified under bulk electrolysis.

Historically, the PC phenomenology originated through observations of under-potential deposition, where incipient electrode surface - electrolyte ion interactions induce surface dipoles - the strength of which is proportional to the difference in the chemical potentials. The kinetic barriers for adsorption may thus be reduced. The consequent surface-adsorbate interactions may 
also facilitate ion reduction/oxidation and metal deposition at the monolayer level at relatively lower applied potentials[295].

Such dipole like interactions could contribute to PC, as exemplified through experimental observations on the surfaces of two-dimensional films. For example, noble metal films, e.g., Au, Ir, $\mathrm{Pt}, \mathrm{Pd}, \mathrm{Rh}, \mathrm{Ru}$, etc. are susceptible to oxidation which occurs over a range of potentials and is surface area dependent[297]. As evidenced in $\mathrm{RuO}_{2}$ films[296], the oxidation of the $\mathrm{Ru}$ ion species to different degrees of oxidation could be manifested as PC [296]. It is worthy to note that the observed CV plot (Section 3.1.1) - see Figure 46 (also see Section 3.1) is reminiscent of double layer characteristics with a rectangular current -potential curve shape (also see Figure 10 in Section 3.1.1). The behavior observed in Figure 46 could be understood broadly in terms of the gradual oxidation (in the anodic scan) and reduction (in the cathodic scan) of the Ru surface over a range of potentials. From the ratio of the net area of the I-V curve to the scan rate, the analysis of the accumulated charge implied a change of the oxidation state of the Ru from 0 (in the elemental form) to 4 (as $\mathrm{RuO}_{2}$ ) over the scanned voltage range. In aqueous solutions, the formation and dissolution of hydrogen would also need to be considered. Indeed, proton diffusion through the formed oxide has been cited as a contributing factor to the conductivity of the $\mathrm{RuO}_{2}$ [294]. However, on repeated cycling to voltages above $\sim 1.4 \mathrm{~V}$ (with reference to the standard hydrogen electrode), a stable $\mathrm{RuO}_{2}$ layer is formed, which may then undergo a gradual surface area dependent reversible reduction and oxidation. Such behavior is manifested in the multiple curves of Figure 46, where a reverse voltage sweep immediately induces a current of the opposite polarity. The accompanying redox reaction was postulated to be[296]: $\mathrm{RuO}_{2}+2 \mathrm{H}^{+}+$ $2 \mathrm{e}^{-} \Leftrightarrow \mathrm{Ru}_{2} \mathrm{O}_{3}+\mathrm{H}_{2} \mathrm{O}$. 
The decay of the PC typically follows the overpotential decay curve (see Section 3.3.1) or diffusional characteristics. For example, thickness dependent voltage profiles were observed in the case of $\mathrm{RuO}_{2}$ through successive galvanostatic charge/ discharge cycles (Section 3.3.1). While thick films of $\mathrm{RuO}_{2}$ (formed by thermal oxidation of $\mathrm{Ru}$ ) indicated a close to full recovery on charging after the discharge: Figure 47 (a), the recovery was reduced for thin films: Figure 47 (b). Such behavior was ascribed to the initial discharge of the near-surface region of the $\mathrm{RuO}_{2}$ followed on charging by the easier redistribution of the oxidation state (e.g., due to electron or proton hopping) in the thinner films. The maintenance of the higher oxidation state yields a successively smaller potential drop on continued cycling.

In the context of CV (see Section 3.1), redox capacitance may sometimes be manifested through sharp peaks, $c f$., Figure 9 (b), whenever there are phase changes in the adsorption process. The peaking of the capacitance over this range (as also discussed later in Figure 46, is due to two competing factors, i.e., the rising part of the curve is due to the exponential rate of reaction rate as the applied voltage is increased, while the falling part is due to the coverage of the adsorbate tending to a monolayer.

A discontinuous change of the chemical potential as a function of the extent of surface coverage $(\theta$, where $0<\theta<1)$ - as discussed later with reference to Eqn. (50), may also give rise to sharp maxima/minima around the transition corresponding to the potential change. Even in this case the reversibility could be maintained, at scan rates $(s)$ close enough to maintain equilibrium. However, the degree of irreversibility was considered to be a function of the $s / k[298]$. With increasing scan rate, the anodic and cathodic peaks shift in the direction of the scan and the peak separation increases. It can be derived that the peak voltage varies as $\ln$ (scan 
rate) corresponding to a Tafel-like regime, where only the forward or backward reactions are significant[136]. It is instructive to compare the PC spectra as a function of the $s / k$ ratio (see Figure 48) to observe the onset of irreversibility[299]. At a sufficiently large $s$, (say, >100 $\mathrm{V} / \mathrm{s}[136])$ the duration of the voltage at which redox reactions can occur exceeds the time scale necessary for species from the bulk to make their way onto the electrode and subsequent reactions to occur, and only the influence of adsorbates that are already present on the electrode/substrate can be evaluated. It was then found that the $s \square k$ ratio should be approximately $<0.01$ for reversible processes, while the ratio should be $>1$ for irreversible process; Figure 48. Additionally, adsorbate interactions, variable surface coverage and other microscopic factors (see Section 5.1.2) may also contribute to irreversible behavior. It is interesting to note that the irreversibility in the anodic and cathodic sweep cycles in Figure 48 (d) corresponds to that of a battery-like behavior, as will also be discussed later with reference to Figure 52.

Considering specific cases of surface processes contributing to the PC, surface oxidation is more reversible on Ru electrodes[296], compared to that on metals such as Au (reversible for $\square \theta \leq 0.02$ ), Pt (reversible for $\square \theta \leq 0.15$ ), etc. However, the degree of irreversibility again increases with increasingly anodic potentials, seemingly proportional to the oxidation state of the $\mathrm{Ru}$ (either in the $\mathrm{Ru}^{4+} / \mathrm{Ru}^{3+}$ states) culminating in a stable oxide. Plotting the $C_{P C}$ as a function of $s \square k$ would then give a measure of the maximum charge/discharge rate of the pseudocapacitance that could be harnessed from a given adsorbate reaction.

\section{1 .1}

\section{Criteria for maximal}

pseudocapacitance 
Fundamentally, PC based characteristics may be attributed to surface enhanced adsorbate-electrode interactions (exceeding mutual adsorbate-adsorbate interactions) and preferential charge transfer. Consider, for example the adsorption of an ion $\left(A^{-}\right)$on a substrate $(M)$, under constant current conditions, with $\theta_{A}(<1)$ being the fractional degree of coverage of the adsorbate $\left(M A_{a d s}\right)$ interpreted through the extent to which electrochemical reactions could occur on a particular surface, and (1- $\square \theta_{A}$ ) being the coverage of the reacting $A^{-}$species, of the form:

$$
A^{-}+M \Leftrightarrow M A_{a d s}+e^{-}
$$

The extent of coverage $\left(\theta_{A}\right)$ is parameterized, and can be experimentally quantified, as a function $\int^{t_{2}} i d t \int_{V_{1}}^{V_{2}} i d V$

of time $(t) /$ voltage $(V)$ through: $\theta_{A}=\frac{t_{1}}{Q}=\frac{V_{1}}{Q \cdot V}$, where $Q$ is the charge per monolayer of adsorbate at full coverage and $s$ is the voltage scan rate. With $\theta_{A}=\frac{q_{A}}{Q}$, where $\theta_{A}$ is the reacting charge as a fraction of the total charge on the surface $(Q)$, a differential capacitance corresponding to the PC, can now be defined as:

$$
C_{P C}=\frac{d q_{A}}{d V}=Q \frac{d \theta_{A}}{d V}
$$

The $C_{P C}$ may then be considered unimportant at very low as well as at very high coverage of the adsorbate. An adsorption equilibrium [300] for the reaction of the form represented in Eqn. (49) would involve the equalities of the forward flux of reaction $\left[Q_{f}=k_{f}\left(1-\theta_{A}\right) I_{A-} \exp \left(\alpha \frac{V F}{R T}\right)\right]$ to a 
backward flux of reaction $\left[Q_{b}=k_{b} \theta_{A} I_{0} \exp \left(-(1-\alpha) \frac{V F}{R T}\right)\right]$, where the $I$ refers to the respective species concentration. The spirit of the above forms follow from the activation complex mediated derivation of the Butler-Volmer equation[137] - see Sections 3.1.2 and 4.3, where $\alpha \square \square$ is the transfer coefficient for a forward/oxidative reaction while (1- $\alpha \square \square$ is the transfer coefficient for the backward/reductive reaction. At equilibrium, with $Q_{F}=Q_{B}$, and with an equilibrium constant, $k$ (compounded from the ratio of the forward equilibrium constant $\left(k_{f}\right)$ and the backward equilibrium constant $\left(k_{b}\right)[138]$ ), at a constant driving potential of magnitude $V$, results in an equation of the form:

$$
\left(\frac{\theta_{A}}{1-\theta_{A}}\right)=k I_{A^{-}} \exp \left(\frac{V F}{R T}\right)
$$

Inserting Eqn. (51) into Eqn. (50) and simplifying, this would yield a capacitance per unit area (with the units of $\mathrm{F} / \mathrm{cm}^{2}$ ) [126] :

$$
C_{P C}=\frac{Q F}{R T} \theta_{A}\left(1-\theta_{A}\right)
$$

From a mathematical standpoint, the $C_{P C}$ would be maximized for $\theta_{A}=0.5$, and equal to:

$$
\left[C_{P C}\right]_{\max }=\frac{Q F}{4 R T}
$$

The aspect that $C_{P C}$ is maximum at $\theta_{A}=0.5$ is intriguing and merits further investigation of the basic mechanisms, in the context of attractive interaction of the electroactive species with the substrate. The $\theta_{A}\left(1-\theta_{A}\right)$ term is also reminiscent of entropic interactions[301], where the highest entropy at $\theta_{A}=0.5$ may correspond to an optimized free energy configuration. For a 
surface charge density, $Q$ of the order of $10 \% / \mathrm{cm}^{2}$, the maximum pseudocapacitance obtained would be of the order of $1.6 \mathrm{mF} / \mathrm{cm}^{2}$ at $T=300 \mathrm{~K}$, two orders of magnitude larger than the nominal double-layer capacitance (see Section 2.3.2). It is tempting to consider that if combined with large surface area nanostructures, such a large capacitance could yield battery-like capacities (also see Section 5.1.3).

\section{1 .2}

\section{Underlying mechanisms}

\section{of adsorption pseudocapacitance}

It has been implied from Eqn. (51) is then that the driving energy (/mole), $V F$, for the surface coverage induced electrochemical reactions, is proportional to $R T \ln \left(\frac{\theta_{A}}{1-\theta_{A}}\right)$. However, the mutual interaction between adsorbates, and the influence of the voltage scan rate $(s)$ have both been ignored in such a formulation. It was also assumed that adsorption occurs due to the greater interaction (with lower free energy) of the reacting species with the substrate compared to mutual interactions. A plot of the coverage, $\theta_{A}$ as a function of the potential is depicted in Figure 49. Identifying the discharge current $C_{P C} d V / d t$ with the Tafel current $\left(i_{F}\right)$ at a given potential $(V)$ through $i_{F},=i_{o} \exp \left(-V / k_{B} T\right)-$ which in turn is derived from the Butler-Volmer equation (Eqn. 36) at large electrode overpotentials, $\eta[80]$, the relevant capacitance is $i_{F} /(d V / d t)$ and the integrated surface charge $(\Delta q)$ between two potentials, $V_{l}$ and $V_{2}$ would be given by:

$$
\Delta q=q_{s} \Delta \theta=\int_{V_{1}}^{V_{2} i_{o} \exp \left(-e V / k_{B} T\right)} \frac{(d V / d t)}{(d t)} d V
$$


The $\Delta q$ is a function of the change in the coverage $(\Delta \theta)$, with $q_{s}$ being the charge associated with a monolayer/ $\mathrm{cm}^{2}$ (= unit charge per the ion/ionic projected area $\sim 1.610^{-19} \mathrm{C} /(0.3 \mathrm{~nm})^{2} \sim 180$ $\mu \mathrm{C} / \mathrm{cm}^{2}$, for a $\mathrm{K}^{+}$ion) may thus be deduced through such analysis of the current-potential and potential decay measurements.

From a more fundamental point of view, adsorption causes the formation of a dipole either due to polar Coulomb interactions or due to non-polar van der Waals interactions[302] both of which could change the surface work function/electron affinity and consequently the rates of chemisorption. The ambient/electrolyte determines the respective dipole orientation, e.g., whether the dipole is pointing (a) negative side, or (b) positive side away from the substrate, and influences the chemisorption. It has been discussed that case (a) holds in the case of hydrogen adsorption[303], whereby electron transfer from the adsorbate would be diminished and the magnitude of released energy on adsorption (/heat of adsorption), $\Delta \square$ would decrease with increasing coverage. The physical rationale is the assumption that the process of adsorption is a consequence of attractive interaction and implies a negative heat of adsorption $(\Delta \square)$, the magnitude of which would be gradually reduced with increasing coverage $(\theta)$. Alternately, case (b) may hold for oxygen adsorption, where the work function is diminished by an amount proportional to the number of dipoles, the strength of the dipole moment, and the extent of coverage. Dipoles may also be formed, e.g., on metallic surfaces due to the kinetic energy of the electrons, and enable their influence to extend slightly above the surface inducing a natural negative charge. The issue of dipole orientation, say, in the case of aqueous solutions, on either side of the $\mathrm{PZC}$, would be sensitive to the $\mathrm{pH}$ and determine intrinsic surface charge polarity (e.g., when the oxygen atom of the $\mathrm{H}_{2} \mathrm{O}$ is oriented towards the electrode surface, in acidic 
environments, the surface would be predominantly $\mathrm{H}^{+}$oriented and hence positively charged). It has been found, e.g., from surface electronic structure sensitive reflectance studies [123], that chemisorbed hydrogen can be either (a) quasi-ionic, through proton binding, or (b) covalently bound to the surface, or even (c) in various localized states (see Section 4.6.1) - each of which may correspond to different binding energies (distributed over a range of $\sim 0.4 \mathrm{eV}$ ) and may be manifested as peaks in potentiodynamic/CV measurements. Such multiple states of adsorption yielding a broad current-voltage profile in the CV (as in Figure 46), has been considered to be relatively common in atom/radical reactions with the substrate and are thought to originate from surface heterogeneity induced through (a) surface orbital hybridization and consequent adsorbate-surface reconstruction, (b) modulation of the substrate properties due to the adsorbates, (c) adsorbate orientation and coordination - due to dipole rearrangement, (d) long range interactions between the adsorbates, etc., being some of the reasons. Further extensive characterization, based on electrical measurements (i.e., surface polarization and dielectric constant measurements, surface conductivity, etc.), as well as magnetic measurements (i.e., surface paramagnetism) would yield greater specific understanding of the states of adsorption.

Generally, $\Delta \square$ would need to be added to the free energy $(=V F)$ to obtain the net energy barrier [304]. The decreasing $\Delta \square$ with increasing coverage may be manifested through a relation of the form: $\Delta \square(\theta)=\Delta \square(\theta \square \square \square \square)-r \cdot \theta$, where $\Delta \square(\theta \square \square \square \square)$ is the heat of adsorption of the pristine surface - at zero coverage, and $r$ is a positive coefficient of decrease. Assuming that the entropy change on adsorption is negligible, the free energy/electrochemical potential would also be enhanced/diminished by an amount $r \cdot \theta$, hence modifying Eqn. (51) by a multiplicative term proportional to $\exp (-g \theta)$, where $g=r / R T$, yielding an equation of the form: 


$$
\frac{\theta}{1-\theta}=k I_{A^{-}} \exp \left(\frac{V F}{R T}\right) \exp (-g \theta)
$$

There may also be a variation in $r$ due to (i) a distribution of active sites with a range of activation energies, e.g., different varieties and charges of the defects (see Section 4.8.2), (ii) dispersion forces - both attractive and repulsive between the adsorbate-substrate, between adsorbates at higher $\theta \square$ etc. A greater variation of binding/adsorption could have the consequence of expanding the voltage range over which adsorption and electrochemical reactions occur[126]) with the undesirable attribute that $C_{P C}$ would be reduced: Figure 49. Additionally, they may lead to a nonlinear variation of $\Delta H$, say, of the form:

$$
\Delta \square(\theta)=\Delta \square(\theta \square \square \square \square)-r \theta^{\mathrm{n}}
$$

Such a form has to be considered at larger values of $\theta \square$ when dipole repulsions are important (yielding $\mathrm{n}=1.5$ ), when mutual ionic interactions in the adsorbed layer dominate (with $\mathrm{n}=0.5$ ), or through the agency of London dispersion forces[304] (with $n=3$ ). Such effects are manifested through an asymmetric capacitance-voltage profile[263] - also see Figure 51. There is also a concomitant nonlinear change of $\theta$ with increased $r$. For chemical heterogeneity at the surface, the $\theta \square$ would need to be specific to the surface site, e.g., for edge planes and basal planes on graphite, $\theta_{\text {basal }}$ vs. $\theta_{\text {edge }}$ must both be considered (see Section 4.8.2.2), each with their specific $r$ coefficients. Taking into account the variation of the $\Delta \square(\theta \square)$ to the free energy in addition to the coverage dependent change in the potential energy, the net free energy driving the adsorption $(\Delta G)$ would be: 


$$
\Delta G=V F+R T \ln \left(\frac{\theta}{1-\theta}\right)+r \cdot \theta
$$

Consequent to considering adsorption phenomena, the current flux would be proportional to exp $(-\Delta G / R T)$, where the forward current flux $\left(Q_{f, a d s}\right)$ and the backward current flux $\left(Q_{b, a d s}\right)$ due to the adsorption ( $a d s)$ would be (also see discussion related to Eqn. (51) and Sections 3.1.2):

$$
\begin{gathered}
Q_{f, a d s}=k_{f}\left(1-\theta_{A}\right) I_{A-\exp }\left(\frac{\alpha V F}{R T}\right) \exp \left(-\frac{(\Delta G-\beta r \theta)}{R T}\right) \\
Q_{b, a d s}=k_{b} \theta_{A} I_{0} \exp \frac{-(1-\alpha) V F}{R T} \exp \frac{-\Delta G+(1-\beta) r \theta}{R T}
\end{gathered}
$$

The $\alpha(/ 1-\alpha) \square$ and the $\beta(/ 1-\beta) \square$ are the respective transfer coefficients for a forward (/backward) bulk chemical reaction and for the forward (/backward) surface chemical reactions. Equating $Q_{f, a d s}=Q_{b, a d s}$, the adsorption isotherm is then modified to a form of Eqn. (59). For reversible processes, the $C_{P C}=Q \frac{d \theta_{A}}{d V}$ (cf. Eqn. 52) is changed to[123]:

$$
C_{P C}=\frac{Q F}{R T} \frac{\theta_{A}\left(1-\theta_{A}\right)}{1+g \theta_{A}\left(1-\theta_{A}\right)}
$$

From Eqn. (59), a $g<0$ would yield a larger value for the $C_{P C}$, for a given value of $\theta$. While the conventional understanding of the $C_{P C}$ assumes that $g$ (or $r$ ) $>0$, it is also intriguing to consider whether the opposite case $(g<0)$ is plausible, i.e., where the presence of adsorbate would be expected to enhance $\Delta \square \square$ with increasing $\theta \square \square \square$ This would occur for example, when the adsorbates are mutually attracted, overcoming the interactions with the substrate/electrode. 
However, the range of negative $g$ values would also be limited, conferring limited benefits, i.e., when mutually attracted, the adsorbates could be described as a more continuous dielectric charge storage layer but the extent of continuity would be at most confined to one monatomic layer and hence limited. These aspects are revealed in the shape of the capacitance-voltage profiles (see Figure 50) where $g<0$ values result in sharper peaked curves at lower potentials (due to more concentrated electric fields), compared to the contrary $g>0$ case, where there is a displacement in the profile. A large negative $g$ may then correspond to the formation of solid phases, instead of a monolayer[299]. The dipole induced change in the $\Delta \square$, which has been previously described through double layer kinetics, further decreases the stability of the adsorbed ions[305] due to mutual repulsion.

It was also seen that the PC is generally a function of the applied potential, as the $\theta$ varies exponentially with the applied voltage $(V)$ : as in Figure 46. From the proportionality of $V F$ to $R T \ln \left(\frac{\theta}{1-\theta}\right)+r \cdot \theta$ : Eqn. (57), and from the definition of $C_{P C}$ : Eqn. (50), we get two contributions to the $C_{P C}$, i.e., (a) one proportional to $\frac{F}{R T} d \theta / d(\ln [\theta / 1-\theta)$ - a coverage dependent Langmuir capacitance $\left(C_{\text {Lang }}\right)$ - in close correspondence to $\left[C_{P C}\right]_{\max }-$ see Eqns. (52) and (53), and (b) another proportional to $F / r$ - termed a Temkin capacitance $\left.\left(C_{T e m}\right)[263,304]\right)-$ which is potential independent (however, when $r \neq 1$ for the $\Delta \square(\theta)$ variation, $C_{\text {Temkin }}$ would vary as $\theta \square^{1-r}$ [263]). As the $C_{\text {Lang }}$ and $C_{T e m}$ add in series, their individual effects and magnitudes are reflected in the net $C_{P C}$, with the smaller capacitance dominating. Given typical values of $r$ of the order of $10-20 \mathrm{kcal} / \mathrm{mole}(\sim 0.4-0.8 \mathrm{eV}), C_{T e m}$ seems to be a primary contributor to diminishing the $C_{P C}$ from the Langmuir/coverage dependent capacitance dominated values of $\sim 1 \mathrm{mF} / \mathrm{cm}^{2}-$ 
see Eqn. (53). Since any adsorbate would be expected to possess a finite value of $r$, the decrease of the $C_{P C}$ is inevitable, and a reduction of the net $C_{P C}$ to $\sim 0.1 \mathrm{mF} / \mathrm{cm}^{2}$ with $r \sim 0.86 \mathrm{eV}$ has been estimated. Indeed, it can be deduced, that now:

$$
\left[C_{P C}\right]_{\max }^{\text {Lang+Tem }}=\frac{Q F}{4 R T+r}
$$

Hence, the $C_{T e m}$ (through the $r$ term) often determines the maximum PC that can be obtained from a given structure. A large $r$ also implies a greater reduction of the energy on species adsorption and may be understood in the sense of tending towards a conventional ionic double layer capacitance (Section 2.3.1), where the Coulomb induced adsorption may be taken as one extreme of energy reduction. Consequently, the capacitance is manifested over a larger potential range, as the "n" - from Eqn. (56) increases, as seen from Figure 51. It was also determined that the width of the potential would scale linearly with $r$ [263]. The shape of the consequent capacitance-potential curve - see Figure 51 [263], is nominally symmetric, with (i) a peakedness or kurtosis determined by the $r$ value, and (ii) a skewness decided by the nonlinear variation of $\Delta \square(\theta \square)$ due to interactions and energy overlap of the adsorbates[263]. In summary, the Langmuir contribution to the PC corresponds to a potential dependent adsorption: per Eqn. (52), without consideration given to the interaction of the adsorbates, the Temkin contribution considers the interactions and generally determines the range of potentials over which the PC may be manifested. A quantitative analysis of the experimental capacitance-voltage curves could then be used [263] for the estimation of the various parameters, such as the $r$ as well as the roughness factor (which is defined to be the ratio of the observed value of the pseudocapacitance to the maximum value, say at $\theta \square \square \square \square \square \square \square$. 


\section{1 .3}

The relation of

pseudocapacitance to battery-like behavior

It was seen from Figure 48, that at extremely large scan rate/chemical rate constant values the manifestations of the PC may be considered analogous to battery-like behavior. Indeed, a comparison was made with the cyclic voltammogram of a $\mathrm{Pb} / \mathrm{PbCl}_{2}$ battery (see Figure 52)

Also, considering that the PC involves electron transfer between the surface and adsorbates over a narrow voltage range, analogies to a redox reaction - as in the case of batteries, can be made. For instance, Faradaic redox conversion process, may be written in terms of the surface concentrations of the oxidized species $\left(I_{O}^{S}\right)$ and the reduced species $\left(I_{R}^{S}\right)$ in the form of a Nernst relation (also see Eqn. 13):

$$
E=E_{o}+\frac{R T}{z F} \ln \frac{I_{O}^{S}}{I_{R}^{S}}=E_{o}+\frac{R T}{z F} \ln \frac{I_{O}^{S}}{I_{O, c}^{S}-I_{O}^{S}}
$$

The $I_{O, c}^{S}$ is the surface concentration of the species, corresponding to complete oxidation. Eqn. (61) can be written in an equivalent form: $\left(\frac{I_{O}^{S}}{I_{O, c}^{S}-I_{O}^{S}}\right)=\exp \left(\frac{\left(E-E_{o}\right) z F}{R T}\right)$, similar to Eqn. (51), from which can be deduced an equivalent capacitance, 


$$
C_{P C, \text { redox }}=\frac{z F}{R T} \frac{Q_{O}}{Q_{O, c}}\left(Q_{O, \mathrm{c}}-Q_{O}\right)
$$

$Q_{o}$ and $Q_{O, c}$ are the charge densities corresponding to $I_{O}^{S}$ and $I_{O, c}^{S}$. Proceeding as before, for $\theta_{o}$ $=0.5 \theta_{M}$, the $\left[C_{P C, \text { redox }}\right]_{\max }=\frac{z F Q_{O, \mathrm{c}}}{4 R T}-$ similar in form to Eqn. (53).

While it was previously discussed (Section 5.1.1) that for a surface charge density of $10^{15} / \mathrm{cm}^{2}$, the maximum PC obtained would be of the order of $1.6 \mathrm{mF} / \mathrm{cm}^{2}-$ two orders of magnitude larger than the nominal double-layer capacitance, which when combined with large surface area nanostructures, could yield battery-like capacities. However, it is now concluded that the mutual interaction between the adsorbates as well as with the substrate yields an order of magnitude lower values, see for example: Figure 51.

\section{1 .4}

\section{Intercalation}

\section{pseudocapacitance (Inner \& Outer Electrode Areas?)}

Thus far, the PC contributions to the net capacitance and charge storage have been discussed as arising mainly from surface electrochemical reactions and adsorbate interactions[306], as in the case of $\mathrm{RuO}_{2}$ (Section 5.1). However, a major drawback is that only the surface is involved, with obvious limitations on the enhancement of the overall energy capacity. In this context, it would be interesting to explore whether the internal area in open/porous structures could also be involved in the PC. That this was indeed possible was seemingly shown in charge transfer in crystalline oxides, such as $\mathrm{Nb}_{2} \mathrm{O}_{5}$ and termed as intercalation pseudocapacitance [173]: Figure 53. Such intercalation could involve charge 
transfer through the bulk of the crystal (and be more closely related to batteries) and reactions on surfaces within the bulk. The relevant redox reaction: $\mathrm{Nb}_{2} \mathrm{O}_{5}+x \mathrm{Li}^{+}+x \mathrm{e}^{-} \leftrightarrow \mathrm{Li}_{\mathrm{x}} \mathrm{Nb}_{2} \mathrm{O}_{5}$, converting $\mathrm{Nb}^{5+}$ to $\mathrm{Nb}^{4+}$ and vice versa, and involving $\mathrm{Li}^{+}$intercalation, was thought to be facilitated in open layered structures, with adequate free space to facilitate $\mathrm{Li}^{+}$diffusion/motion. X-ray absorption spectroscopy was used to monitor the oxidation state of the $\mathrm{Nb}$ and indicated increasing peak energies with increasingly positive cell potentials, implying a greater degree of electron filling/increased $\mathrm{Nb}^{5+}$ to $\mathrm{Nb}^{4+}$ reduction. Other characteristics of intercalation pseudocapacitance were listed as: (1) The linearity of the measured current to the scan rate $(s)$, whereas in diffusion based systems the current would vary as the $\sqrt{s}$, (2) the charge storage/capacity would be constant with charging time: Figure 53 (b), presumably because only surface processes are involved, and (3) that there is no significant shift of the redox peak potentials with increased $s$, implying that the relevant redox reactions (related to the $\mathrm{Nb}^{5+}$ to $\mathrm{Nb}^{4+}$ interconversion) are reversible. Consequently, aided through the PC mechanism, charging rates larger than that of traditional battery anodes was observed: Figure 53(c).

The related electrochemical experiments were done through drop-casting a sonicated solution of $\mathrm{Nb}_{2} \mathrm{O}_{5}$ in ethanol onto a stainless-steel foil in a three-electrode setup (with lithium foils as counter and reference electrodes) and using as electrolyte $1 \mathrm{M} \mathrm{LiClO}_{4}$ dissolved in propylene carbonate (PC). The assertion that $\mathrm{Li}^{+}$ions do intercalate into the $\mathrm{Nb}_{2} \mathrm{O}_{5}$ structure was based on (a) the distinction of $\mathrm{CV}$ curves when $1 \mathrm{M} \mathrm{LiClO}_{4}$ in $\mathrm{PC}$ solvent was used - wherein faint redox peaks were present in contrast to the case where $0.1 \mathrm{M}$ tetrabutylammonium perchlorate (TBAP) in PC was adopted - wherein only curves symptomatic of double layer capacitance (i.e., rectangular in shape, as discussed in Section 3.1.1). It was asserted that the larger size of the TBA ion $(>0.3 \mathrm{~nm})$ in comparison to the $\mathrm{Li}$ ion $(<0.1 \mathrm{~nm})$ prevented the former 
from intercalation into the $\mathrm{Nb}_{2} \mathrm{O}_{5}$. However, the employing of a faster scan rate $(100-500$ $\mathrm{mV} / \mathrm{s}$ ) when using the TBAP, and the absence of a discussion of whether intercalation barriers are involved (e.g., the question of whether the process of de-intercalation proceeds with a similar rate constant as the insertion process) makes direct comparison difficult.

The intercalation PC based mechanisms are in contrast to the redox reactions occurring in battery systems, as there may be no significant phase and related volume change in the former. As discussed earlier in Section 2.2.1, such changes are a major cause of (a) slow rates of charging, as well as (b) the gradual onset of irreversibility, in battery-related systems. However, the reported gradual change of the $\mathrm{Nb}-\mathrm{O}$ bond length from $\sim 0.14 \mathrm{~nm}$ to $\sim 0.19 \mathrm{~nm}$, over the course of changing potentials, as well as the gradually increasing cathodic peak shift may be interpreted as a change in the crystal characteristics and raises the question of what would be termed as distinct phases and the extent of phase change, since most practical phase change processes in nature are of the second order where a particular phase gradually merges into another. Since the authors propose intercalation PC as a distinct electrochemical process of charge/energy storage, it would definitely be imperative to consider whether the proposed bulk intercalation is really identical to surface adsorption, as the former involves redox reactions on surfaces inside the bulk material. Related issues which seem to merit further investigation are: (a) whether intercalation without a distinct phase change is evident, considering the extent to which a redox peak should be manifested in $\mathrm{CV}$ to be characteristic of such intercalation effects, (b) the possible effects of intrinsic/extraneous states on contributing to the redox peak (e.g., as seen in the peak near $\sim 1.4 \mathrm{~V}$ in the Fig. 3 of the supplementary information of Reference [173]), and (c) whether there would be an activation barrier for the ion intercalation, etc. 
In another related study, it was indicated that the presence of ions in the electrode similar to those in the electrolyte (e.g., $\left.\mathrm{Na}_{2} \mathrm{SO}_{4}\right)$ might enhance ionic (e.g., $\mathrm{Na}^{+}$ions) diffusion, through invoking a pre-intercalation mechanism[307] in $\mathrm{Na}^{+}$ion impregnated $\mathrm{MnO}_{2}$ compound based nanosheets. Redox peaks (perhaps, relevant to the process: $\mathrm{MnO}_{2}+x \mathrm{H}^{+}+y \mathrm{Na}^{+}+(x+y) \mathrm{e}^{-} \leftrightarrow$ $\mathrm{MnOOH}_{\mathrm{x}} \mathrm{Na}_{\mathrm{y}}$ ), overlaid on the double layer capacitance based background were observed in $\mathrm{CV}$ scans. While the reported capacitances (of the order of $1 \mathrm{~F} / \mathrm{cm}^{2}$ or $1000 \mathrm{~F} / \mathrm{g}$ ) energy density (of $110 \mathrm{Wh} / \mathrm{kg}$ ) and power density (greater than $1 \mathrm{MW} / \mathrm{kg}$ ) seem impressive, the relevant tests were carried out in a three electrode setup[120] - see Section 2.4.3. It is also not apparent why diffusion of $\mathrm{Na}^{+}$would be enhanced, for the redox reactions, when the concentration gradient would plausibly be reduced between the $\mathrm{Na}_{\mathrm{x}} \mathrm{MnO}_{2}$ electrode and the electrolyte. The enhanced $\mathrm{Na}^{+}$diffusion, was justified by a calculated diffusion coefficient of $3.07 \cdot 10^{-6} \mathrm{~cm}^{2} / \mathrm{s}$ which was much higher than a previously reported values of $\sim 10^{-12}$ to $10^{-14} \mathrm{~cm}^{2} / \mathrm{s}$ for solid state diffusion in $\mathrm{Na}_{0.7} \mathrm{CoO}_{2}$ and $\mathrm{Na}_{0.7} \mathrm{MnO}_{2.25}$, respectively[308]. The orders of magnitude increase in the diffusion coefficient is quite surprising and may be subject to errors, e.g., due to an inaccurate knowledge of the active area (as also suggested by the authors - see Supplementary Information of [307]) as well as the supposition that the redox peaks are due to the $\mathrm{Na}^{+}$diffusion, while they may actually be due to the Mn related defect phases. The influence of disorder (structural as well as stoichiometric) in the synthesized $\mathrm{Na}_{0.7} \mathrm{MnO}_{2}$ has not been well characterized in this study and may well be responsible for the observed effects - as may be evidenced by the increasing irreversibility observed in the CV scans at enhanced scan rates.

An analogy could be drawn between the proposed intercalation PC mechanisms to earlier discussion a few decades ago, on the inner and outer active surface areas in $\mathrm{RuO}_{2}$ based electrode systems[309]. The terminology was related to relatively less accessible (e.g., charge 
stored in surface heterogeneities, such as pores or cracks) or more accessible regions on the oxide, respectively. A representative redox reaction: $\mathrm{RuO}_{\mathrm{x}}(\mathrm{OH})_{\mathrm{y}}+\delta \mathrm{H}^{+}+\delta \mathrm{e}^{-} \leftrightarrow \mathrm{RuO}_{\mathrm{x}-\delta}$ $(\mathrm{OH})_{\mathrm{y}+\delta}$, served for proton $\left(\mathrm{H}^{+}\right)$diffusion and storage on the surface of the hydrated $\mathrm{RuO}_{2}$. However, it was noted that a different amount of charge could be stored in alkaline and acidic background media, as a $25 \%$ enhancement in the charge capacity was observed at $\mathrm{pH}=14$ compared to when $\mathrm{pH}=0$ presumably due to a higher oxidation states on the surface $\mathrm{Ru}$ ions in the former case. It was found concomitantly that the measured charge $\left(Q_{\text {meas }}\right)$ varied with scan rate $(s)$ as $Q_{\text {meas }}=c_{1}+\frac{c_{2}}{\sqrt{s}}$, with $c_{1}$ and $c_{2}$ as numerical constants, and justified on the basis that $s$ varied inversely with the diffusion time. The maximum available surface charge $\left(Q_{\max , S}\right)$ was $a$ priori partitioned to be the sum of the charges available from the inner surface area $\left(Q_{i n, S}\right)$ and the outer surface area $\left(Q_{\text {out }, S}\right)$, i.e., $Q_{\max , S}=Q_{i n, S}+Q_{\text {out }, S}$. At high $\left.s \square \square \square \rightarrow \square \infty\right)$, the inner areas would be unavailable implying that case $Q_{\text {meas }}\left(=q_{\max , S}, s \square \square \square \square \infty\right)=Q_{\text {out }, S}$. The same data formed the motivation for the writing of another relationship of the form: $\frac{1}{Q_{\text {meas }}}=c_{3}+c_{4} \sqrt{s}$,

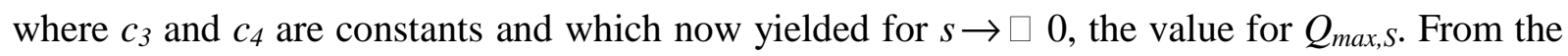
determination of $Q_{\max , S}$ and $Q_{\text {out }, S}$, the quantity $Q_{i n, S}$ as well as the ratio of the inner to the outer electrode areas was estimated. As it was then found that the inner area was approximately $25 \%$ smaller than the outer area - in close correspondence to the numbers for the charge capacity difference as a function of the $\mathrm{pH}$, inferences were drawn on the diffusion mechanisms of the $\mathrm{H}^{+}$ ions. In acidic conditions (low $\mathrm{pH}$ ), the oxide surface is relatively positively charged hindering ion diffusion considering only the outer active surface areas. However, ion diffusion was promoted accessing the entire oxide area and enhancing the $\mathrm{RuO}_{\mathrm{x}}(\mathrm{OH})_{\mathrm{y}}$ redox process in 
alkaline (high $\mathrm{pH}$ ) backgrounds. Consequently, the charge capacity was explained to be higher in the latter case. While such rationale purported to explain experimental observations, it was subject to obvious criticism on using the same experimental data to obtain two different functional relationships and which could have led to "fantastic conclusions"[310]. Indeed, a later paper by the authors[311] states that considering the case of " $s \square=0$ is often less ambiguous than the extrapolation to $s \square \square \square \square \infty$ ”. Indeed, “s $\{\square \square \square \infty$ " based cases are physically unrealistic, and reports of "infinite sweep rate capacitance" [173]do not seem to be scientifically valid or technologically relevant. It was also though that the fitted linear increase of the charge capacity with $\sqrt{s}$ was not adequately borne out in experiments[310].

Consequently, there are still many questions on whether enhanced energy storage or power density can be obtained through intercalation based PC. Typically, the rate performance of batteries decreases with increased discharge (/charge) rate due to the limitations of diffusion. Surface based redox capacitance could indeed be an alternative if all the necessary ions (for the relevant redox processes) are at the surface. However, the presence of the double layer and the consequent reorganization time (which affects the diffusion coefficient, $D$ ) must be considered, in addition to the diffuse layer where the reservoirs of redox contributing species reside (Section 2.3.1). Since the diffusion time varies as $l^{2} / D$ intercalation based diffusion into the crystal structure, say over a distance of $\sim 1 \mathrm{~nm}$ can be approximated to be orders of magnitude faster than diffusion of the species from the diffuse layer of $\sim 100 \mathrm{~nm}$ [79]. Consequently, the enhanced rate of discharge (/charge) should be manifest over at most an order of magnitude in the charging/C-rate. Nevertheless, prospects of obtaining battery like performance in ECs make such investigations worth pursuing. 
We will further consider, in the next section, further approaches to diminish the diffusive component through the optimized arrangement of nanostructures.

\section{storage in nanoscale confined electrolytes}

Approaches to enhance the energy and power density in tandem have also considered methodologies to reduce the ion-diffusive component, e.g., through a Faradaic process based redox capacitance[294] harnessed via transition metal oxides (of which $\mathrm{RuO}_{2}$ is a good example) on high surface area nanocarbons. The rationale is that larger currents $(i)$ could be obtained through a linear dependence on the voltage scan rate $(s)$ in Faradaic processes, compared to the $s^{1 / 2}$ dependence characteristic of diffusion. However, while the gravimetric capacitance could be large[312] (e.g., of the order of $720 \mathrm{~F} / \mathrm{g}$ for $\mathrm{RuO}_{2}[294]$ the low electrical conductivity of oxides typically implies low rate capability[294]. An alternative energy storage mechanism may be proposed, whereby diffusion (both in the horizontal and vertical directions) could be precluded through (a) ensuring that the spacing between the nanostructures would be constituted of a charged electrolyte, and (b) where the charge is stored in the confined electrolyte. Such stagnant electrolyte impregnated electrodes could provide a new paradigm for charge and energy storage.

Charge storage may then be realized through the use of high surface area nanostructured materials, such as activated carbon (AC) and carbon nanotubes (CNTs)[313-315] - also see 
Section 4.8.1, in conjunction with redox electrolytes. Optimized pore diameter or spacing mitigates diffusional limitations and could yield high charge/discharge rates without a detrimental effect on the cycling stability, which is often an issue when using surface functional groups[294] for PC based storage (see Section 5.1.2 and Figure 51). Concomitantly, a combination of redox electrolytes, e.g., KI (for the positive electrode) and vanadyl sulfate ( $\mathrm{VOSO}_{4}$, for the negative electrode) was investigated[316,317], with AC as the electrodes. However, it was seen that the charge storage capacity was diminished at high discharge rates. Computational modeling of Faradaic process in porous electrodes has found that reduced capacity may be due to planar diffusion to the exterior surface[318] and not within the pores of the electrode itself.

It was also intriguing to consider that Faradaic processes in porous materials may possess superior kinetics otherwise absent in bulk materials.[319] Such electrocatalytic effects could arise from thin layer electrochemistry (TLE) based processes. [320,321] For TLE to prevail[322], the nanostructure spacing or pore diameter would need to be smaller than the equivalent diffusion layer thickness $(\delta)$ :

$$
\delta \sim \sqrt{\pi D t} \sim \sqrt{\pi D^{\Delta V} / s}
$$

$D$ is the relevant ion diffusion coefficient, and the corresponding voltage range is $\Delta V$ ). The charge contained in the enclosed electrolyte could then be harnessed through Faradaic reactions due to homogenous depletion/accretion of redox species. In addition to the enhanced kinetics, due to the absence of diffusion, the capacity would be directly proportional to the electrolyte 
volume confined in the pore $\left(V_{\text {pore }}\right)$ and its bulk concentration $\left(I_{o}\right)$. It has been derived that the accompanying TLE based peak currents $\left(i_{T L E}\right)=\frac{F^{2}}{4 R T} s V_{\text {pore }} I_{o}$, would vary directly with $s$ [79].

At a low scan rate (say, $s_{1}$ ): see Figure 54, implying a large $\delta$, the diffusion layers at the top of the macro-electrode overlap giving rise to a planar diffusion limited current $\left(i_{\text {diff }}\right)$, proportional to $I_{0}$, the projected area $\left(A_{p r o j}\right)$ of the electrode and $s^{1 / 2}$. Additionally, there would be a TLE based current $\left(i_{T L E}\right)$ due to the electrolyte trapped within the pores, which would be proportional to the $V_{\text {pore }}$ and $s$. At moderate scan rates (say, $s_{2}$ ) the individual $i_{\text {diff }}$ contributions at the top of the nanotube array begins to get resolved. The resulting concentration gradients internal to the pores yields an additional $i_{\text {diff }}$ while the $i_{T L E}$ current contribution reduces. At very high scan rates (say, $\left.s_{3}\right)$, there would be a dominant $i_{\text {diff }}$ proportional to the net area $\left(A_{n e t}\right)$ of the electrode owing to a complete decoupling of diffusion layers and the CNTs behaving as individual nanoscale electrodes. The amount of charge (or capacity) contributed by the solvated redox species confined in the $V_{\text {pore }}$, in the TLE regime is given by $Q=z F V_{\text {pore }} I_{o}$, where $z$ is the number of electrons per redox reaction. Consequently, high capacity and rate capability may be realized in the TLE regime through designing electrodes with large $V_{\text {pore }}$ and small $\delta$.

While activated carbon based materials may possess a large active area $\left(>2000 \mathrm{~m}^{2} / \mathrm{g}\right)$, their $V_{\text {pore }} \sim 0.5-1 \mathrm{~cm}^{3} / \mathrm{g}$ would result in a smaller magnitude of accessible capacitive charge[111]. It would then be pertinent to explore the capacitive characteristics, in the TLE regime, of a model macroporous electrode comprised of vertically aligned CNTs with a spacing corresponding to an equivalent pore size and a larger $V_{\text {pore }}\left(\right.$ of $\sim 40 \mathrm{~cm}^{3} / \mathrm{g}$ ). To this end, the electrochemical performance of the CNT array electrodes in redox electrolytes, i.e., $\mathrm{K}_{3} \mathrm{Fe}(\mathrm{CN})_{6}$ or $\mathrm{Ru}\left(\mathrm{NH}_{3}\right)_{6} \mathrm{Cl}_{3}$ 
was characterized using CV (Section 3.1) through the variation of the cathodic (or anodic) peak currents: $i_{p}$, with respect to the $s$ :Figure 55. From the average CNT spacing of $\sim 350 \mathrm{~nm}$ and with $0.04 \mathrm{~V} / \mathrm{s}<s<4 \mathrm{~V} / \mathrm{s}$, and $\Delta V \sim 26 \mathrm{mV}(=R T / F)$ the $\delta$ was estimated to be $\sim 3.7 \mu \mathrm{m}$ even at the largest scan rate. As the $\delta$ was significantly larger than the nanotube spacing, the system could exhibit TLE behavior under the given conditions.

The current observed in CV could have contributions from both (a) planar diffusion limited current at the top of the electrode $\left(i_{\text {diff }}\right)$ and $(\mathrm{b})$ a TLE current $\left(i_{T L E}\right)$ in between the CNTs. For a reversible, one-electron redox reaction, over a time, $t$, the total peak current, $i_{p}$ (after baseline correction) is[79]:

$$
i_{p}=i_{\text {diff }}+i_{T L E}=F A_{\text {proj }} I_{o}\left(\frac{\pi D F}{R T}\right)^{1 / 2} \chi\left(\frac{F s t}{R T}\right) \cdot v^{1 / 2}+\left(\frac{n^{2} F^{2}}{R T}\right) \frac{\Upsilon}{(1+\Upsilon)^{2}} V_{\text {pore }} I_{o} \cdot s
$$

In Eqn. (64), $\chi\left(\frac{F s t}{R T}\right)$ denotes the normalized current for a reversible reaction under planar diffusion while $\Upsilon=e^{\frac{\left(E-E^{o}\right) F}{R T}}$ where $E$ is the applied voltage and $E^{0}$ is the formal potential of the redox couple (see Section 3.1.1). The deconvolution of a particular I-V curve, obtained from CV, into the thin layer (black curve) and the diffusion constituents (red curve) is depicted in Figure 55 (b). While it's possible to estimate $A_{\text {proj }}$ and $V_{\text {pore }}$ for a given electrode using Eqn. (64), the voltammetric currents at any given potential may not necessarily follow the relation, due to uncompensated resistance induced ohmic distortion[323]. To separate Ohmic and mass transport effects, the current-voltage voltammograms were numerically computed from the rate expression previously derived by de Tacconi[324]. Figure 55 (c) compares the thin layer currents $\left(i_{T L E}\right)$ with (red curve) and without (black curve) Ohmic distortion. It was seen that the distorted peak was 
lower amplitude but smaller but wider compared to the undistorted peak. However, if the Faradaic reaction is allowed to completion, i.e., if the voltammetric peak is well within the scanned potential window, the voltammetric charge $Q_{t o t}$ will be unaffected by Ohmic effects. Consequently, analysis of $Q_{t o t}$ could provide greater insights into the kinetics.[325]

$$
Q_{\text {tot }}=Q_{\text {diff }}+Q_{T L E}=\frac{\int_{V_{i}}^{V_{f}}\left(i_{\text {diff }}+i_{T L E}\right) d V}{s}=F A_{\text {proj }} I_{o}\left(\frac{\pi D F}{R T}\right)^{1 / 2} \int_{V_{i}}^{V_{f}} \chi\left(\frac{F s t}{R T}\right) \cdot s^{-1 / 2} d V+F V_{\text {pore }} I_{o}
$$

A key aspect related to TLE regime is that the electrochemical response seems to be identical to that of species adsorption on the electrode from the linearity of the observed $I$ with the $s$. Such species adsorption may be undesirable and form a rate-limiting step if the corresponding kinetics are slow. However, the measured capacitance may be treated as Faradaic due to the absence of mass transport and the confinement of electroactive species on or close to the surface[136,326].

To evaluate the capacity of the examined electrode/electrolyte system, galvanostatic discharge experiments were carried out with different concentrations of the redox additives at a constant current density. A large plateau was observed in the voltage at close to the formal potential $(\sim 0.23 \mathrm{~V}$ vs. SCE) of the redox couple indicative of Faradaic/battery-like characteristics: Figure 56 . The recorded charge capacity, in $\mathrm{mAh} / \mathrm{cm}^{3}$ or $\mathrm{mAh} / \mathrm{cm}^{2}$, was obtained through a chronopotentiogram, by dividing the charge by either $V_{\text {pore }}$ or $A_{\text {proj, }}$, respectively. The discharge current density was $\sim 200 \mathrm{~mA} / \mathrm{cm}^{2}$ (corresponding to the ratio of the applied current of $50 \mathrm{~mA}$ and $\left.A_{\text {proj }}\right)$. For $1 \mathrm{M} \mathrm{K} \mathrm{K}_{3} \mathrm{Fe}(\mathrm{CN})_{6}$, the maximum observed capacity, from : Figure 56 (a), was $\sim 26 \mathrm{mAh} / \mathrm{cm}^{3}$ or $0.28 \mathrm{mAh} / \mathrm{cm}^{2}$. Such values were close to or relatively better (considering the discharge current densities) than those previously observed and estimated for macroporous 
electrodes constituted from, e.g., (1) Nickel foam based electrodes with $\mathrm{CoO}[327]: 0.8 \mathrm{mAh} / \mathrm{cm}^{2}$ (at $40 \mathrm{~mA} / \mathrm{cm}^{2}$ ), (2) hybrid Polypyrrole/CoO nanowire array[328] of $0.15 \mathrm{mAh} / \mathrm{cm}^{2}$ (at 50 $\left.\mathrm{mA} / \mathrm{cm}^{2}\right)$, (3) conductive hybrid metal oxides $\left(\mathrm{Ni}(\mathrm{OH})_{2} / \mathrm{NiCo}_{2} \mathrm{O}_{4}\right)$ on carbon fiber paper: 0.25 $\mathrm{mAh} / \mathrm{cm}^{2}$ (at $150 \mathrm{~mA} / \mathrm{cm}^{2}$ )[329], (4) $\mathrm{Co}_{3} \mathrm{O}_{4} / \mathrm{NiO}$ core/shell nanowire arrays[330]: $\sim 0.18$ $\mathrm{mAh} / \mathrm{cm}^{2}$ (at $120 \mathrm{~mA} / \mathrm{cm}^{2}$ ), (5) $(\mathrm{Co}, \mathrm{Ni}$ ) based compounds on hollow core nanorod array[331]: $\sim 0.14 \mathrm{mAh} / \mathrm{cm}^{2}$ (at $10 \mathrm{~mA} / \mathrm{cm}^{2}$ ), etc. It was also observed from the inset of Figure 56 (a), that the observed capacity was linearly proportional to the concentration of the redox species, with the implication that a further improvement in capacity would be possible using redox additives with a greater solubility, e.g., KI, with a solubility limit of $\sim 8 \mathrm{M}$ [332]. Moreover, the capacity normalized to the thin layer capacity (through the calculated $V_{\text {pore }}$ ) seems to be constant beyond $50 \mathrm{~mA} / \mathrm{cm}^{2}$ : of Figure 56 (b), implying that much of the observed capacity (at high current densities) is within the electrolyte confined in $V_{\text {pore }}$. At lower current density, diffusional contributions would also add to the capacity. It was noted that the TLE based capacity could be cycled at very high rates for up to 5000 cycles while exhibiting excellent Coulombic efficiency, i.e., $Q_{\text {discharge }} / Q_{\text {charge }}$ of $\sim 97 \%$. Further work in increasing the capacity could focus on electrolytes with a greater concentration of the redox species as well as electrodes with an increased pore volume. While such approaches to harness large density in EC based constructions need to be pursued actively, there has also been considerable work in alternate materials and cell architectures. Such aspects will be discussed next. 


\section{and prospects}

A major imperative for continuing research in charge storage is the exploration of new storage mechanisms as well as alternate materials systems where such mechanisms could be harnessed. We have seen, for example, that carbon based materials are ubiquitous, i.e., as graphite based anodes in battery systems - Section 2.2.2, and as activated carbon electrodes in ECs - Section 2.3. The advantages of an intrinsic layered nanostructure in the former and large surface area in the latter are the relevant nanoscale features. In this section, we will briefly explore the use of alternate materials and device architectures, that may be relevant for future charge storage applications. As the possible use of nanocarbons, e.g., one-dimensional carbon nanotubes (CNTs) and two-dimensional graphene has been previously considered (Section 4.8.1), we will first focus on polymeric materials (which offer the possibility of obtaining new insights into charge transfer at the molecular level, in addition to a reduced cost imperative). The section will conclude through surveying a few new trends in charge storage architecture with respect to miniaturized energy and power sources.

6.1

Electroactive

\section{polymers for high charge density and capacitance}

Presently, there is high commercial interest in organic materials related technologies, as their lower cost makes them attractive for large-scale deployment, covering portable consumer electronics, military applications and electric vehicles and other applications. Electroactive 
polymers: EAPs[333], are then suitable candidates for electrochemical capacitors with the dual advantages of (i) a large $C_{d l}$ (Section 2.3.1) due to large porosity and surface area, as well as (ii) high values of the Faradic capacitance or $C_{p}$ (Section 5.1) from the multitude of reaction sites, specific to each monomer. The relevant electrochemical charge/discharge processes, i.e., doping/de-doping, are fast and charge can be stored through the volume of the material[334,335]. Indeed, a common thought is that the space between the polymer chains allows complete access to the bulk. However, the space is typically small enough to disallow the formation of a double layer and may be responsible for high polymer electrode and electrode-electrolyte resistance[336], as observed in EIS (Section 3.3).

Organic molecules such as quinone $\left(\mathrm{C}_{6} \mathrm{H}_{4} \mathrm{O}_{2}\right)$, capable of storing two electrons per molecular unit and with a molecular weight of $108 \mathrm{~g}$ would have a charge density of 2 Faradays/108 g equivalent to $1787 \mathrm{C} / \mathrm{g}$ or $496 \mathrm{mAh} / \mathrm{g}[337]$, comparable to state-of-the art electrodes using in battery systems - see Sections 2.2.2 and 2.4.1. Keeping such considerations in mind, many conjugated polymers have been synthesized bearing redox-active units[338]. A summary of common EAPs and their properties is presented in Table II. The upper limits of specific energy (see Section 2.4) are roughly equal for the EAPs, at $~ 300 \mathrm{Wh} / \mathrm{kg}$, promising even higher storage than inorganic electrodes. However, as will be discussed later, polymer stability related issues yields much lower energy densities of the order of $10 \mathrm{Wh} / \mathrm{kg}$.

Table II Charge storage characteristics for a range of generic electroactive polymers.[333] 


\begin{tabular}{|l|l|l|l|l|}
\hline Types of Polymer & $\begin{array}{l}\text { Charge Density } \\
(\mathrm{Ah} / \mathrm{kg})\end{array}$ & $\begin{array}{l}\text { Specific Energy } \\
(\mathrm{Wh} / \mathrm{kg})\end{array}$ & $\begin{array}{l}\text { Open Circuit Voltage } \\
(\mathrm{V})\end{array}$ & $\begin{array}{l}\text { Specific Power } \\
(\mathrm{W} / \mathrm{kg})\end{array}$ \\
\hline Polyacetylenes & $100-300$ & $100-300$ & $3.5-3.9$ & up to 30,000 \\
\hline Polyaniline & $50-150$ & $100-350$ & $1-4$ & \\
\hline Polypyrrole & $50-120$ & $50-350$ & $3-4$ & \\
\hline Polythiophene & $25-100$ & $50-325$ & $3.2-4.2$ & up to 90,000 \\
\hline Poly-p-phenylene & $20-140$ & 300 & $3.2-4.5$ & up to 320 \\
\hline
\end{tabular}

Of the typical materials in Table II, polyanilines and polyacetylenes suffer greatly from solubility problems and instability, whereas polythiophenes offer the best advantages in terms of ease of functionalization, solubility and therefore processability. Polyacetylenes provide the highest charge storage capacity, but problems associated with trace metals (i.e., Al/Ti) left over from the Ziegler-Natta synthesis leads to increased degradation of charge storage. It is therefore essential to adapt methodologies to give clean polymer materials free from residual catalyst and side-products. In terms of the development of new EAP forms, the recent combined use of highly redox-active entities, e.g., the polythiophene chain and tetrathiafulvalene (TTF) allows an exceptionally high loading of positive charge with the advantages and characteristics of a typical conjugated polymer (film forming properties, flexibility, and semiconducting behavior). The TTF unit was chosen as an additional redox component of the polymers due to its well-known reversible two-electron oxidation[339][340].

Given the relatively low surface area for double layer formation and high electrical resistance, conducting polymer dispersions (where the polymer swelling is stabilized as negatively charged colloidal particles which may then be cross-linked through cations) have been proposed as constituents of a porous network based electrode. For example, a swollen hydrogel constituted from PEDOT [poly(3,4-ethylene dioxythiophene)] - PSS [poly(styrenesulfonate)] Baytron ${ }^{\circledR}$ particles with an outer negative charge due to $\mathrm{SO}_{4}{ }^{2-}$ ions linked in a network by $\mathrm{Mg}^{2+}$ 
ions has been for a double layer capacitor[336]. The negative and positive ions were introduced into the polymer through equilibration of the PEDOT-PSS with $0.25 \mathrm{M} \mathrm{MgSO}_{4}$ for about two hours. While swelling is prevented through the cationic linkages, a higher ionic mobility (yielding lower electrical resistance) is facilitated through swelling by water diffusion into the electrode. However, typical water content in excess of $75 \%$ of the total mass lowers the energy density and additional blending ( $37.5 \mathrm{wt} \%)$ of conducting polymers (e.g., Polypyrrole: PPy) was advocated to enhance the electrical capacitance. A large power density (of $\sim 10 \mathrm{~kW} / \mathrm{kg}$ ), without a significant compromise in the energy density (at about $7 \mathrm{Wh} / \mathrm{kg}$ ) [336], could be harnessed through the agency of swelling and the addition of the conducting polymer. While environmental effects considerably affect the electrochemical characteristics of EAPs, a major imperative still remains the ubiquity of polymers in natural forms, a prime example of which is the use of lignin - a byproduct of the paper/pulp industry, and which has recently received much attention for its possible use in charge storage[341] as wooden batteries[342].

\subsection{1}

\section{Lignin modified}

\section{electrodes}

Lignin, a complex biopolymer, can be cheaply synthesized (at the level of $\$ 0.10 / \mathrm{kg}$ ) through the alkaline, acidic, or organic decomposition of wood chips, and has been proposed as substrates for electrochemical reactions. While alkaline reagents yield an increased number of nonphenolic $-\mathrm{OH}$ and sulfide groups (through the Kraft process[343]), reactions with acidic mixtures yield water-soluble lignosulfonates (LS). Alkali lignins exhibit strong adsorption on gold electrodes (of the order of $3 \cdot 10^{14}$ units $/ \mathrm{cm}^{2}$ ) [344], as well as on insulating substrates[343], 
and subsequent oxidation in sulfuric acid results in a restructured redox-active polymer with quinone-active functionalities [344]. A linear dependence of the measured current in CV: Figure 57 (which is also typically used to measure the charge capacity: Section 3.1) with scan rate was indicative of a surface confined process (see Section 3.1.1) - as in Figure 57 (C).

LS based polyanions have also been used to dope other polymers, such as polyaniline (PANI) [343] or polypyyrole (PPy) [341] and regulate the mechanical plasticity. For example, LS have been employed as "negative plate expanders" in increasing the cold cranking performance of lead acid batteries[345]. In this regard, several LS constituent groups has been implicated in enhancing and preserving the electrical capacity (mediated through the carboxyl groups) and cycle lifetime (through the phenolic hydroxyl groups) and improved cold cranking (through the methoxyl groups), while sulfur groups have been shown to have a negative influence. The activity of multiple redox centers in lignin-derived films may be confirmed through differential pulse voltammetry[79]. Generally, the adsorption of the LS and the complexation with the metallic $\mathrm{Pb}$ was shown to enhance the effective surface area for electrochemical reaction. Indeed, the increase of the active area seems to be a principal effect of the LS compounds.

Consequently, such material can be considered as a prototype for surface active/pseudocapacitive (see Section 5.1) reactions, where diffusion control plays a minimal role, as the negatively charged sulfonic groups constitute a barrier (/sink) for negative (/positive) charges from the electrolyte. The degree of sulfonation and the barrier seems to decrease with increasing molecular weight[343]. Together with the tendency of the sulfonic groups to complex with metal ions, a decreased molecular weight was imputed in an increase in the 
electrocatalytically observed currents. Such aspects are also manifested in the very limited frequency range over which diffusion plays a role, as observed in EIS (see discussion related to Figure 17 in Section 3.3). However, the charge-transfer resistance $\left(R_{c t}\right)$ : Eqn. (24), is quite large and composed of contributions from multiple redox reactions. Two particular processes that have been identified relate to electron transfer (i) at the LS-electrode interface and (ii) in the LS.

Generally, modified lignin polymers are constituted of in-chain or pendant groups capable of redox reactions and can be tailored to investigate surface specific processes for use in chemical- and bio-sensors, enhanced electrocatalytic activity, etc. As an example, it was shown that a lignin based redox couple was a good mediator for the electrochemical oxidation of the ascorbic acid through diminishing the overpotential by $\sim 0.25 \mathrm{~V}[344]$. Given the relatively open structure of lignin, substituent addition may be used to control the redox potential, e.g., the addition of electron donating oxygen moieties or carboxylic acid groups, or alternately increasing the $\mathrm{pH}$, would also contribute to reducing the lignin redox potential. The activity of the added substituents is directly related to the proximity of the substituent to the central phenyl group, which is a major determinant of the lignin redox potential. An electron-withdrawing (/additive) group, on the phenyl ring, would induce a positive (/negative) shift. Figure 58 indicates another relevant example, [341] where redox reactions typical to quinone groups in a PPy matrix composite electrode are shown. The water in an aqueous electrolyte systems serves as the proton donor/acceptor to the quinone moiety and it is pertinent to note that such electroactivity is absent in non-aqueous systems.

However, the tenability of polymers as electrodes is fraught with issues related to the stability as well as those related to the lack of understanding of the polymer structure interactions 
- both in the bulk and on the surface. As just one example of the complications involved with modeling the electrochemistry at relatively insulating surfaces[346], hitherto unaccounted for states, possibly at the surface (with a density of $\sim 10^{13}-10^{14} / \mathrm{cm}^{2}$ ), were thought to possess cryptoelectrons with a higher reducing potential compared to the bonding electrons[347]. The action of such cryptoelectrons was seemingly manifested in the enhancement of the $\mathrm{pH}$, hydrogen evolution, reduction of $\mathrm{Fe}(\mathrm{CN})_{6}{ }^{3-}$ to $\mathrm{Fe}(\mathrm{CN})_{6}{ }^{4-}$, etc., indicating the necessity of electron transfer as opposed to ion transfer. The origin of such states, also observed through triboelectric contact between polymers, were ascribed to [347]: (1) intended dopants or unintended impurities, (2) surface states formed by mechanical forces, (3) end groups with reactive radicals forming molecular ion-like states, or even (4) cosmic ray induced ionization. Such issues serve to indicate the lack of understanding as well as complications inherent to the practical use of polymers in charge storage. The charge/discharge kinetics would also be spatially dependent[348], regulated by the presence of such not well understood nanoscale states and would have to be carefully controlled[349].

\subsection{2}

\section{Polymer - Inorganic}

\section{oxide hybrids}

The potential of oxides as capacitors, in terms of enhanced redox capacitance, has previously been discussed (see Section 5.1). However, it was also discussed that a low electronic conductivity handicaps their power capability. Consequently, various nanostructured conductive polymer-oxide hybrids have been synthesized with the objective of overcoming this issue. For example, PANI- $\mathrm{V}_{2} \mathrm{O}_{5}$ hybrid electrodes with, a three fold larger energy and power density 
compared to the individual materials and, capacities of $\sim 320 \mathrm{mAh} / \mathrm{g}$ (with a current density of $0.5 \square \mathrm{A} / \mathrm{cm}^{2}$; see Figure 56 for a comparison of current densities) have been synthesized through layer-by-layer assembly of the two components[350]. The volume expansion typical of ion intercalation into the oxide (Section 2.2.1) was accommodated through the use of a porous PANI nanofiber based network. It is interesting to note that such hybrids accommodate two distinct redox reactions, viz., one related to the intercalation/de-intercalation of ions $\left(e . g ., \mathrm{Li}^{+}\right.$ions in $\mathrm{LiClO}_{4}$ electrolyte; Section 2.2.3) into the oxide, and the other related to the doping/de-doping of anions in the PANI. Generally, surface redox processes (Section 5.1) seem to be pertinent for PANI while diffusion related processes held for the $\mathrm{V}_{2} \mathrm{O}_{5}$ redox reactions, as deduced from the current-scan rate relationships (see Section 3.1.2). However, the validity of the partitioning of the total charge transfer to inner and outer constituents of the PANI nanofibers, in this study, is suspect (as discussed in detail previously in Section 5.1.4) considering that the paths of access and the influence of multiple layers, which have not been well specified. A large capacity fading over a limited number of cycles $(<100)$ indicates the environmental susceptibility of the polymer-oxide hybrids, as well. Consequently, polymeric materials and their incorporation into new types of charge architectures and devices, for the purpose of charge storage, need much more study with respect to their stability and ultimate feasibility with respect to their cost advantages.

6.2 
Considering the seemingly never-ending need for powering portable electronics, compact high-energy sources would continue to be developed. While alternative sources, such as solar power, etc. have been much touted due to possible limitations with materials and minerals, electrochemical sources have been widely studied over the past century and have been considered to be relatively reliable and stable.

For example, with electrochemical capacitors (Section 2.3), we have seen that the charge capacity is directly proportional to the area and provides an imperative for increasing the geometric area (Section 2.3.2 and 5.2) or the effective area through surface chemical reaction (Section 5.1) However the area increase should not be accompanied by an increase of the device volume, given the imperative for compact energy sources. A few recognized ways to accomplish this have been previously discussed, e.g., through an increase in the pore area in electrodes (see Section 4.9) and/or using thin layer electrochemistry (see Section 5.2) to reduce the resistance associated with diffusion/mass transport. Alternate ways to accomplish such an area increase has been suggested through the incorporation of three-dimensional architectures (e.g., see Figure 59), and designs into compact electrochemical devices[351] while keeping the effective electrode thickness small (which aids increased power density).

A major initial motivation seems to be integration of such compact devices with micro/nano-electro mechanical systems (N-/M-EMS), ranging from smart dust motes[351] to ubiquitous wireless sensors[352]. Many 3-D electrode architectures may be fabricated through bottom-up approaches, well understood for MEMS based applications, and involving material deposition (often through electrochemical methodologies) into porous membranes, e.g. anodic aluminum oxide based templates. Given that the areal capacity, rather than the volumetric 
density, may be an important metric also for 3-D batteries[353] the required value for competitive designs was estimated to be of the order of $10 \mathrm{mAh} / \mathrm{cm}^{2}$, while practical state-of the art $\mathrm{Li}$ thin film batteries only seem to provide $1 \mathrm{mAh} / \mathrm{cm}^{2}$, (equivalent to $3.6 \mathrm{~J} / \mathrm{cm}^{2}$ at $1 \mathrm{~V}$ ), indicating the need for substantial further development.

A scientific imperative was suggested to be the shorter ion diffusion lengths that would result in having a more accessible surface area, and which would enhance the power density. Individually separated electrodes, as depicted through the inter-digitated scheme in Figure 59(a) would have a poor performance, due to issues related to non-uniform current distributions from the variable distance between the anode and the cathode. Also, an initial comparison of such 3-D architectures with 2-D thin film/plate like designs may just imply a folding of the latter to the former yielding the electrode area increase, with a consequent increase of the electrolyte volume and possibly lowered energy density. However, there would be a length scale (in the height/third dimension) beyond which the gain in energy density would be larger in 3-D designs. For maintaining uniform current distribution, in a given contributing individual electrode area $(A)$ through such length $(L)$ increase, a dimensionless number related to $\left(\frac{A \mu R}{L}\right) Q$ was defined for a given volumetric charge density $\left(Q: \mathrm{C} / \mathrm{m}^{3}\right)$ in terms of the ion-mobility $(\mu)$ as well as the electrode resistance $(R)$. A decreasing $U$ corresponds to a more uniform charge distribution along the electrode length and enhances the efficiency of a given 3-D scheme. Considering an assemblage of such optimally designed electrodes, alternating placement, e.g., in which an anode (/cathode) is surrounded by six cathodes (/anodes) may provide for more uniform current density at the anode (/cathode). However, it was shown[354] that it would be very difficult to achieve homogeneous lithiation/delithiation (Section 2.2.1) due to preferential current density 
concentration at the electrode tips. While such differential current densities at the electrodes are prevalent to separated cathodes and anodes, they may be avoided through the use of conformal designs. 
Consequently, the conformal deposition of the electrodes and electrolyte, as in Figure 59(b)-(d), may present interesting alternatives. The latter constrain diffusion to be onedimensional in character, as is the case with traditional lithium ion batteries: Figure 3, with the implication of higher ionic transport and increased charge transfer efficiency. However, additional issues related to the uniformity, stability, as well as the thickness of the electrolyte have been considered challenges for future development of 3-D batteries[353]. The latter aspect is critical in that the ion transport through the electrolyte remains the power limiting step and it would be desirable to have a very thin layer while ensuring a high supply of ions, i.e., the electrolyte concentration should be sufficiently high (> 1M), from Debye length considerations: see Eqn. (7). Fundamental electronic considerations would also dictate the lower limits of the thickness, i.e., either from an electrostatic point of view, where there may be overlap of the Helmholtz/Stern double layers (Section 2.3.1) which may enhance/diminish ion movement, or from a quantum mechanical point of view where tunneling between the electrodes (Figure 25) should be avoided, as such charge transfer would effectively connect the electrodes. Additional aspects related to the electrochemical and mechanical stability of the electrolyte vis-à-vis the electrodes as well as the limiting transport of the $\mathrm{Li}^{+}$ions [355] - which have been noted to have a transference number of less than 0.5 , seem to be factors limiting the durability in terms of the number of cycles (typically $<50$ ), in present 3-D battery architectures.

An interesting idea in this regard, seems to be a consolidated/monolithic glassy solid-state cell[355]: Figure 60. Given the deep knowledge base and understanding of glass formation, a network former common to the electrode and electrolyte could provide a uniform ambient for the $\mathrm{Li}^{+}$transport and considerably reduce interfacial polarization effects. However, whether such architecture could provide adequate separation and the necessary chemical potential difference 
between the anode and the cathode (each of which would be constituted from the redox potentials of distinct transition metals) still needs to be investigated in detail. Similar ideas are involved in the proposal of an "all-solid-state-battery", where the electrodes and electrolytes were all constituted from the Lithium phosphate family[356], e.g., the positive and the negative electrodes were $\mathrm{LiFePO}_{4}$ and $\mathrm{Li}_{3} \mathrm{~V}_{2}\left(\mathrm{PO}_{4}\right)_{3}$, while the solid electrolyte was $\mathrm{Li}_{1.5} \mathrm{Al}_{0.5} \mathrm{Ge}_{1.5}\left(\mathrm{PO}_{4}\right)_{3}$. Stacks of the electrode and electrolyte layers were fused together through carefully designed spark plasma sintering based process - with control over the granularity of the precursor materials and the composite electrode formulations which were shown to produce crack-free laminates with high quality contiguous interfaces. The quality of the electrode/electrolyte interfaces was ensured through noting that the cell resistances even decreased on repeated cycling. A residual porosity (of $\sim 15 \%$ ) together with lubricant like carbon additive was thought to be responsible for accomplishing local stress relaxation and ensuring material integrity. Such technological advances seem to allow for relatively thick electrodes (of the order of 0.3-0.8 mm) yielding a high surface energy capacity ranging to $10 \mathrm{mAh} / \mathrm{cm}^{2}$ - which realizes a long sought after goal for high energy densities. However, later experimental work [357] yielded 2.2 $\mathrm{mAh} / \mathrm{cm}^{2}$ with a plateau voltage of $2.45 \mathrm{~V}$. However, the advantage of developing such new schemes for battery design lie in the gestation of new ideas and their possible subsequent incorporation, e.g., through (i) structured surfaces where the large area particular to nanostructures, (ii) continuous electrodes and discontinuous/isolated electrolyte, (iii) random, yet percolating, networks of electrodes and electrolytes, yielding a sponge like design: Figure 59.(d)) etc. Hierarchical designs that combine high surface area (through the use of nanostructures) combined with large volumes (e.g., through the growth of nanostructures in porous foams) would also be beneficial for harnessing large capacitance as well as large volumes of electrolyte. The 
use of electrically conductive aerogels (comprising both carbon and transition metal oxide based constituent materials) may be attractive in designing meso- and macro-porous size distributions and have been used for high power density supercapacitor applications.

However, additional surface area also brings along issues related to unwanted chemical reactions, the tolerance to which must be carefully considered. A relatively quick comparison of reviews [353] and scientific papers[358] over the past decade seems to imply that the "field of 3D batteries is still very much an emerging area of research"[352][353], with energy densities hovering around $1 \mathrm{mAh} / \mathrm{cm}^{2}$ and typifies some of the issues relevant to moving to smaller scales in terms of electrical connectivity and parasitic reactions.

In this paper, the aim was to survey the principles behind the storage of charge and its manifestation as electrical energy in nanostructured materials. The initial discussion was focused on where the charge could be stored in terms of a traditional understanding of nanomaterials with a large surface area to volume ratio. It was seen that a large surface has the advantages of quickly storing and releasing the charge, of much utility in capacitive storage. However, more charge with a concomitant large energy density could be manifest through battery-like devices, where the charge is stored throughout the volume of the material. This set the stage for investigating the fundamentals of energy storage with an emphasis on mechanisms 
involving Li-ion batteries and electrochemical capacitors (ECs). It was seen that while the former relied on charged species transfer and discharge through diffusion into the electrodes, the performance of the latter hinged on the absence of diffusion (of ions in the electrolyte and charge carriers in the solid electrode) through the formation of a very thin double-layer, as typified through the Helmholtz model. Indeed, diffusional consideration lowered the performance of an EC through the serial addition of capacitances, e.g., Gouy-Chapman and a space charge capacitance. Additional lowering of the net EC capacitance was obtained through considering the discrete energy levels in nanostructured materials, where dimensionality effects and concomitant finite density of states results in a series quantum capacitance. Specific to charge transduction devices such as dye-sensitized solar cells is yet another nanoparticle size dependent chemical capacitance.

Particular examples of the materials constituting the electrodes in both batteries and ECs were discussed. In the former category, the difference in the electrochemical potentials of the anode and cathode was directly related to the voltage that could be obtained. In ECs the nature of the electrode-electrolyte interface dictated the charge transfer and Faradaic reactions, which could further modulate the capacitance that could be obtained. The intrinsic carrier concentration and extrinsically induced defects in the electrode must both be considered at the nanoscale. Integral to the performance of both batteries and ECs is the electrolyte, which for quick energy delivery should have a low electrical resistance. Recent advances in electrolyte systems, e.g., ionic liquids, were surveyed with respect to their impedance characteristics as well as the ability to sustain a large voltage. Generally, a voltage larger than that available through aqueous systems $(\sim 1 \mathrm{~V})$ is facilitated through organic electrolytes (up to $\sim 3 \mathrm{~V}$ ) and ionic liquids (which could sustain potential differences of greater than $6 \mathrm{~V}$ ). The order of 
magnitude enhancement in the energy and the power density (both of which scale as $V^{2}$ ) is a major imperative for such studies.

Accurate measurement of the charge density and the proper reporting of the consequent energy and power density are integral to evaluating the performance of charge storage devices. A brief survey of methodology, particularly relevant for ECs, was done with respect to the interpretation of time-/frequency-varying current-voltage curves for quantifying the amount of charge, in terms of various elements incorporating capacitance and its release through resistive terms. While elaborate models, to encompass experimental data, may be constructed based on simpler constructs (e.g., the lumped Randles and the transmission line based distributed deLevie models) interpretation of the resulting values is non-trivial and may also be subjective. A major issue in nanomaterials is the device dependent resistance, which may be subject to a large variability. Here again, the investigation of defect induced pathways for electrical current passage (say, in terms of impurity band conduction) may provide insights into lowering the net impedance.

However, increasing the net charge storage capacity and the capacitance is the major goal. Given that most mechanisms of conventional EC are due to the series addition of capacitances, the stage is set for the probing of parallel charge storage mechanisms. While Faradaic or pseudocapacitive means seem to add two orders of magnitude larger charge capacity due to external or internal surface area of oxides such as $\mathrm{RuO}_{2}$, facilitated through surface adsorbate induced redox reactions, much effort needs to be expended to understand the in situ adsorbate interactions. The invoking of a Temkin capacitance, which relates to the energetics of the surface coverage of adsorbates and which may substantially reduce the pseudocapacitance is 
a case in point. Nevertheless, such ideas may provide insights into truly obtaining high energy and power densities from the same material.

It may be concluded that the investigation of charge storage and harness in nanomaterials is a fertile field, through which possibly an order of magnitude enhancement in the energy and power density is feasible. However, concerted effort has to be put in to identify alternative additive mechanisms, such as pseudocapacitance to boost the charge density, and optimized nanomaterial packaging to alleviate the electrical resistance. It is thought that the consideration of defects and concomitant energy levels as well as construction of equivalent electrical circuit models would enable more genuine and rapid progress on the scientific and technological fronts.

\section{Acknowledgments}

The authors acknowledge support of the National Science Foundation (NSF): Grant CMMI 1246800. P.R Bandaru also greatly appreciates discussion with Prof. P. Skabara at the University of Strathclyde and the hospitality of Prof. S. Ghosh at the Tata Institute of Fundamental Research, Mumbai, where part of the manuscript was written. 


\section{References}

[1] J.R. Miller, A.F. Burke, Electrochem. Soc. Interface (2008) 53.

[2] A.F. Burke, in:, T.B. Reddy, D. Linden (Eds.), Linden's Handb. Batter., McGraw Hill, New York, NY, 2011.

[3] D.J. MacKay, Sustainable Energy - Without the Hot Air, UIT Cambridge Ltd., Cambridge, UK, 2009. 
[4] M. Hoefer, P.R. Bandaru, Phys. Rev. Lett. ( Rev. (2012).

[5] J. Nichols, H. Saito, M. Hoefer, P.R. Bandaru, Electrochem. Solid-State Lett. 11 (2008).

[6] ISO/TS 27687:2008 - Nanotechnologies -- Terminology and definitions for nano-objects - Nanoparticle, nanofibre and nanoplate.

[7] J. Jamnik, J. Maier, Phys. Chem. Chem. Phys. 5 (2003) 5215.

[8] A.S. Aricò, P. Bruce, B. Scrosati, J.-M. Tarascon, W. van Schalkwijk, Nat. Mater. 4 (2005) 366.

[9] D. Larcher, C. Masquelier, D. Bonnin, Y. Chabre, V. Masson, J.-B. Leriche, J.-M. Tarascon, J. Electrochem. Soc. 150 (2003) A133.

[10] A.R. Armstrong, G. Armstrong, J. Canales, R. Garcia, P.G. Bruce, Adv. Mater. 17 (2005) 862.

[11] S. Datta, Quantum Transport: Atom to Transistor, Cambridge University Press, New York, 2005.

[12] S. Ijima, Nature 354 (1991) 56.

[13] M.I. Kastnelson, Graphene: Carbon in Two Dimensions, Cambridge University press, Cambridge, UK, 2012.

[14] S. Das Sharma, S. Adam, E.H. Hwang, E. Rossi, Rev. Mod. Phys. 83 (2011) 407. 
[15] C. Xu, B. Xu, Y. Gu, Z. Xiong, J. Sun, X.S. Zhao, Energy Environ. Sci. 6 (2013) 1388.

[16] S. Datta, Electronic Transport in Mesoscopic Systems, Cambridge University Press, New York, NY, 1995.

[17] L. Pauling, The Nature of the Chemical Bond, Cornell University Press, Ithaca, NY, 1959.

[18] A.M. Cowley, S.M. Sze, J. Appl. Phys. 36 (1965) 3212.

[19] S.M. Sze, K.K. Ng, Physics of Semiconductor Devices, Wiley-Interscience, Hoboken, NJ, 2006.

[20] M.-V. Fernandez-Serra, C. Adessi, X. Blase, Nanoletters 6 (2006) 2674.

[21] W. Dong, J.S. Sakamoto, B. Dunn, Sci. Technol. Adv. Mater. 4 (2003) 3.

[22] J.A. Nichols, H. Saito, C. Deck, P.R. Bandaru, J. Appled Phys. 102 (2007) 64306.

[23] M. a. Hoefer, P.R. Bandaru, J. Appl. Phys. 108 (2010) 034308.

[24] S. Pisana, M. Lazzeri, C. Casiraghi, K.S. Novoselov, A.K. Geim, A.C. Ferrari, F. Mauri, Nat. Mater. 6 (2007) 198.

[25] J.H. Seol, I. Jo, A.L. Moore, L. Lindsay, Z.H. Aitken, M.T. Pettes, X. Li, Z. Yao, R. Huang, D.A. Broido, N. Mingo, R.S. Ruoff, L. Shi, Science (80-. ). 328 (2010) 213.

[26] J. Cai, P. Ruffieux, R. Jaafar, M. Bieri, T. Braun, S. Blankenburg, M. Muoth, A.P. Seitsonen, M. Saleh, X. Feng, K. Müllen, R. Fasel, Nature 466 (2010) 470. 
[27] K.S. Novoselov, Z. Jiang, Y. Zhang, S. V Morozov, H.L. Stormer, U. Zeitler, J.C. Maan, G.S. Boebinger, P. Kim, A.K. Geim, Science (80-. ). 315 (2007) 1379.

[28] Y. Zhou, Q. Bao, L.A.L. Tang, Y. Zhong, K.P. Loh, Chem. Mater. 21 (2009) 2950.

[29] S. Dubin, S. Gilje, K. Wang, V.C. Tung, K. Cha, A.S. Hall, J. Farrar, R. Varshneya, Y. Yang, R.B. Kaner, ACS Nano 4 (2010) 3845.

[30] O.C. Compton, B. Jain, D.A. Dikin, A. Abouimrane, K. Amine, S.T. Nguyen, ACS Nano $5(2011) 4380$.

[31] C.P. Firme, P.R. Bandaru, Nanomedicine 6 (2010) 245.

[32] M. Bockrath, D.H. Cobden, J. Lu, A.G. Rinzler, R.E. Smalley, L. Balents, P.L. McEuen, Nature 397 (1999) 598.

[33] J.H. Poynting, Philos. Trans. R. Soc. London 175 (1884) 343.

[34] D. Linden, T.B. Reddy, Linden's Handbook of Batteries, McGraw Hill, New York, NY, 2010.

[35] M. Winter, R.J. Brodd, Chem. Rev. 104 (2004) 4245.

[36] E.J. Cairns, P. Albertus, Annu. Rev. Chem. Biomol. Eng. 1 (2010) 299.

[37] M.S. Whittingham, Chem. Rev. 104 (2004) 4271.

[38] A. Manthiram, J. Phys. Chem. Lett. 2 (2011) 176. 
[39] J.M. Tarascon, M. Armand, Nature 414 (2001) 359.

[40] J.B. Goodenough, Y. Kim, Chem. Mater. 22 (2010) 587.

[41] J. Christensen, P. Albertus, R.S. Sanchez-Carrera, T. Lohmann, B. Kozinsky, R. Liedtke, J. Ahmed, A. Kojic, J. Electrochem. Soc. 159 (2012) R1.

[42] P.G. Bruce, S.A. Freunberger, L.J. Hardwick, J.-M. Tarascon, Nat. Mater. 11 (2012) 19.

[43] M. Armand, S. Grugeon, H. Vezin, S. Laruelle, P. Ribière, P. Poizot, J.-M. Tarascon, Nat. Mater. 8 (2009) 120.

[44] H. Li, P. Balaya, J. Maier, J. Electrochem. Soc. 151 (2004) A1878.

[45] M. V Reddy, G. V Subba Rao, B.V.R. Chowdari, Chem. Rev. 113 (2013) 5364.

[46] P.L. Taberna, S. Mitra, P. Poizot, P. Simon, J.-M. Tarascon, Nat. Mater. 5 (2006) 567.

[47] P. Poizot, S. Laruelle, S. Grugeon, L. Dupont, J.M. Tarascon, Nature 407 (2000) 496.

[48] W.D. Ryden, A.W. Lawson, C.C. Sartain, Phys. Rev. B 1 (1970) 1494.

[49] P. Balaya, H. Li, L. Kienle, J. Maier, Adv. Funct. Mater. 13 (2003) 621.

[50] I. Bonev, Acta Crystallogr. A28 (1972) 508.

[51] M.M. Thackeray, P.J. Johnson, L.A. de Picciotto, P.G. Bruce, J.B. Goodenough, Mater. Res. Bull. 19 (1984) 179. 
[52] A.R. West, Solid State Chemistry and Its Applications, John Wiley, New York, 1992.

[53] Y.-K. Sun, S.-T. Myung, B.-C. Park, J. Prakash, I. Belharouak, K. Amine, Nat. Mater. 8 (2009) 320 .

[54] A.K. Padhi, J. Electrochem. Soc. 144 (1997) 1188.

[55] D. Danilov, P.H.L. Notten, Electrochim. Acta 53 (2008) 5569.

[56] J. Maier, Prog. Solid State Chem. 23 (1995) 171.

[57] J. Maier, Solid State Ionics 175 (2004) 7.

[58] R.J. Young, P.A. Lovell, Introduction to Polymers, Chapman \& Hall, New York, 1991.

[59] Z. Gadjourova, Y.G. Andreev, D.P. Tunstall, P.G. Bruce, Nature 412 (2001) 520.

[60] G.W.G. A. Kelly, Crystallography and Crystal Defects, TechBooks, Herndon, VA, 1970.

[61] A.M. Christie, S.J. Lilley, E. Staunton, Y.G. Andreev, P.G. Bruce, Nature 433 (2005) 50.

[62] K. Shahi, J.B. Wagner, Appl. Phys. Lett. 37 (1980) 757.

[63] M.L.F. Nascimento, C. Aparicio, Phys. B Condens. Matter 398 (2007) 71.

[64] J. Maier, Nat. Mater. 4 (2005) 805.

[65] S.A. Freunberger, Y. Chen, Z. Peng, J.M. Griffin, L.J. Hardwick, F. Bardé, P. Novák, P.G. Bruce, J. Am. Chem. Soc. 133 (2011) 8040. 
[66] S.A. Freunberger, Y. Chen, N.E. Drewett, L.J. Hardwick, F. Bardé, P.G. Bruce, Angew. Chem. Int. Ed. Engl. 50 (2011) 8609.

[67] A. Rufer, P. Barrade, IEEE Trans. Ind. Appl. 38 (2002) 1151.

[68] P. Simon, Y. Gogotsi, Nat. Mater. 7 (2008) 845.

[69] B.E. Conway, J. Electrochem. Soc. 138 (1991) 1539.

[70] A. Balducci, U. Bardi, S. Caporali, M. Mastragostino, F. Soavi, Electrochem. Commun. 6 (2004) 566.

[71] M. Ohring, Materials Science of Thin Films, 2nd ed., Academic Press, San Diego, 2002.

[72] A. Elahi, D.J. Caruana, Phys. Chem. Chem. Phys. 15 (2013) 1108.

[73] D.C. Grahame, Chem. Rev. 41 (1947) 441.

[74] P. Delahay, Double Layer and Electrode Kinetics, Interscience, New York, NY, 1965.

[75] H. Shi, Electrochim. Acta 41 (1996) 1633.

[76] O. Barbieri, R. Kötz, Carbon N. Y. 43 (2005) 1303.

[77] B.E. Conway, J.O. Bockris, I.A. Ammar, Trans. Faraday Soc. 47 (1951) 756.

[78] T.J. Webb, J. Am. Chem. Soc. 48 (1926) 2589. 
[79] A.J. Bard, L.R. Faulkner, Electrochemical Methods: Fundamentals and Applications, 2nd ed., John Wiley, New York, 2001.

[80] P.H. Rieger, Electrochemistry, 2nd ed., Chapman and Hall, New York, 1994.

[81] H. Gerischer, in:, P.J. Gellings, H.J.M. Bouwmeester (Eds.), CRC Handb. Solid State Electrochem., CRC Press, Boca Raton, FL, 1997.

[82] J.-P. Randin, E. Yeager, J. Electroanal. Chem. Interfacial Electrochem. 36 (1972) 257.

[83] A. Peigney, C. Laurent, E. Flahaut, R.R. Basca, A. Rousset, Carbon N. Y. 39 (2001) 507.

[84] A. Matic, B. Scrosati, MRS Bull. 38 (2013) 533.

[85] M.A. Navarra, MRS Bull. (2013) 548.

[86] A. Brandt, S. Pohlmann, A. Varzi, A. Balducci, S. Passerini, MRS Bull. 38 (2013) 554.

[87] A. Balducci, U. Bardi, S. Caporali, M. Mastragostino, F. Soavi, Electrochem. Commun. 6 (2004) 566.

[88] S.P. Ong, O. Andreussi, Y. Wu, N. Marzari, G. Ceder, Chem. Mater. 23 (2011) 2979.

[89] M. Lazzari, M. Mastragostino, A.G. Pandolfo, V. Ruiz, F. Soavi, J. Electrochem. Soc. 158 (2011) A22.

[90] A.C. Dillon, K.M. Jones, T.A. Bekkedahl, C.H. Kiang, D.S. Bethune, M.J. Heben, Nature 386 (1997) 377. 
[91] J.B. Martin, I.A. Kinloch, R.A.W. Dryfe, J. Phys. Chem. C 114 (2010) 4693.

[92] B.A. Boukamp, J. Electrochem. Soc. 128 (1981) 725.

[93] J.R. Szczech, S. Jin, Energy Environ. Sci. 4 (2011) 56.

[94] M. Nishijima, T. Ootani, Y. Kamimura, T. Sueki, S. Esaki, S. Murai, K. Fujita, K. Tanaka, K. Ohira, Y. Koyama, I. Tanaka, Nat. Commun. 5 (2014) 4553.

[95] C.K. Chan, H. Peng, G. Liu, K. McIlwrath, X.F. Zhang, R.A. Huggins, Y. Cui, Nat. Nanotechnol. 3 (2008) 31.

[96] N. Liu, H. Wu, M.T. McDowell, Y. Yao, C. Wang, Y. Cui, Nano Lett. 12 (2012) 3315.

[97] N. Liu, Z. Lu, J. Zhao, M.T. McDowell, H.-W. Lee, W. Zhao, Y. Cui, Nat. Nanotechnol. 9 (2014) 187.

[98] C.R.M. Grovenor, J. Phys. C Solid State Phys. 18 (1985) 4079.

[99] S.-H. Kang, J.B. Goodenough, L.K. Rabenberg, Chem. Mater. 13 (2001) 1758.

[100] X. Guo, J. Fleig, J. Maier, J. Electrochem. Soc. 148 (2001) J50.

[101] P. Pichanusakorn, P.R. Bandaru, Mater. Sci. Eng. R 67 (2010) 19.

[102] J. Wolfenstine, J.L. Allen, J. Read, D. Foster, Chemistry and Structure of Sony's Nexelion Li-Ion Electrode Materials (ARL-TN-0257, Adelphi, MD, 2006.

[103] C. J., Mater. 6 (2013) 156. 
[104] Q. Fan, P.J. Chupas, M.S. Whittingham, Electrochem. Solid-State Lett. 10 (2007) A274.

[105] F. Wang, R. Robert, N.A. Chernova, N. Pereira, F. Omenya, F. Badway, X. Hua, M. Ruotolo, R. Zhang, L. Wu, V. Volkov, D. Su, B. Key, M.S. Whittingham, C.P. Grey, G.G. Amatucci, Y. Zhu, J. Graetz, J. Am. Chem. Soc. 133 (2011) 18828.

[106] C.-X. Zu, H. Li, Energy Environ. Sci. 4 (2011) 2614.

[107] L. Ji, M. Rao, H. Zheng, L. Zhang, Y. Li, W. Duan, J. Guo, E.J. Cairns, Y. Zhang, J. Am. Chem. Soc. 133 (2011) 18522.

[108] J.-G. Zhang, D. Wang, W. Xu, J. Xiao, R.E. Williford, J. Power Sources 195 (2010) 4332.

[109] B. Kang, G. Ceder, Nature 458 (2009) 190.

[110] Y. Gogotsi, P. Simon, Science (80-. ). 334 (2011) 917.

[111] J. Gamby, P.. Taberna, P. Simon, J.. Fauvarque, M. Chesneau, J. Power Sources 101 (2001) 109.

[112] Q. Lu, G. Keskar, R. Ciocan, R. Rao, R.B. Mathur, A.M. Rao, L.L. Larcom, J. Phys. Chem. B 110 (2008) 24371.

[113] Y. Zhu, S. Murali, M.D. Stoller, K.J. Ganesh, W. Cai, P.J. Ferreira, A. Pirkle, R.M. Wallace, K.A. Cychosz, M. Thommes, D. Su, E.A. Stach, R.S. Ruoff, Science 332 (2011) 1537. 
[114] Y. Zhu, S. Murali, M.D. Stoller, A. Velamakanni, R.D. Piner, R.S. Ruoff, Carbon N. Y. 48 (2010) 2118.

[115] O. Barbieri, M. Hahn, A. Herzog, R. Kötz, Carbon N. Y. 43 (2005) 1303.

[116] S. Brunauer, P.H. Emmett, E. Teller, J. Am. Chem. Soc. 60 (1938) 309.

[117] J. Huang, B.G. Sumpter, V. Meunier, Angew. Chem. Int. Ed. Engl. 47 (2008) 520.

[118] J.P. Olivier, Carbon N. Y. 36 (1998) 1469.

[119] T. Ohba, K. Kaneko, J. Phys. Chem. B 106 (2002) 7171.

[120] M.D. Stoller, R.S. Ruoff, Energy Environ. Sci. 3 (2010) 1294.

[121] R. Holze, Experimental Electrochemistry, Wiley-VCH Verlag GmbH \& Co. KGaA, Weinheim, Germany, 2009.

[122] P.T. Kissinger, W.R. Heineman, (1996).

[123] B.E. Conway, H. Angerstein-Kozlowska, F.C. Ho, J. Vac. Sci. Technol. 14 (1977) 351.

[124] R.G. Compton, C.E. Banks, Understanding Voltammetry, Imperial College Press, London, UK, 2011.

[125] M.A. Hoefer, Electrochemical Implications of Defects in Carbon Nanotubes, University of California, San Diego, 2012.

[126] B.E. Conway, J. Electrochem. Soc. 138 (1991) 1539. 
[127] G. Salitra, A. Soffer, L. Eliad, Y. Cohen, D. Aurbach, J. Electrochem. Soc. 147 (2000) 2486.

[128] L.-B. Zhao, A.K. Mishra, D.H. Waldeck, J. Phys. Chem. C 117 (2013) 20746.

[129] A.K. Mishra, D.H. Waldeck, J. Phys. Chem. C 115 (2011) 20662.

[130] R.S. Nicholson, I. Shain, Anal. Chem. 36 (1964) 706.

[131] A.J. MacLeod, Appl. Math. Comput. 57 (1993) 305.

[132] J. Randles, Trans. Faraday Soc. 44 (1948).

[133] A. Ševčík, Collect. Czechoslov. Chem. Commun. 13 (1948) 349.

[134] H. Matsuda, Y. Ayabe, Zeitschrift Für Elektrochemie, Berichte Der Bunsengesellschaft Für Phys. Chemie 59 (1955) 494.

[135] E. Laviron, J. Electroanal. Chem. Interfacial Electrochem. 101 (1979) 19.

[136] S. Srinivasan, E. Gileadi, Electrochim. Acta 11 (1966) 321.

[137] P.H. Rieger, Electrochemistry, 2nd ed., Chapman and Hall, New York, 1994.

[138] H. Angerstein-Kozlowska, B.E. Conway, J. Electroanal. Chem. Interfacial Electrochem. 95 (1979) 1.

[139] M.E. Orazem, B. Tribollet, Electrochemical Impedance Spectroscopy, John Wiley Inc., New York, NY, 2008. 
[140] E. Barsoukov, J.R. MacDonald, Impedance Spectroscopy: Theory, Experiment, and Applications, Wiley-Interscience, Hoboken, NJ, 2005.

[141] C.M.A. Brett, A.M.O. Brett, Electrochemistry: Principles, Methods, and Applications, Oxford University Press, Oxford, 1993.

[142] B.E. Conway, W.G. Pell, T.-C. Liu, J. Power Sources 65 (1997) 53.

[143] H.B. Callen, T.A. Welton, Phys. Rev. 83 (1951) 34.

[144] J. Bisquert, J. Phys. Chem. B 106 (2002) 325.

[145] R. De Levie, Advances in Electrochemistry and Electrochemical Engineering, Interscience, New York, 1967.

[146] J. Bisquert, G. Garcia-Belmonte, F. Fabregat-Santiago, N.S. Ferriols, P. Bogdanoff, E.C. Pereira, J. Phys. Chem. B 104 (2000) 2287.

[147] D.M. Pozar, Microwave Engineering, 2nd ed., John Wiley \& Sons, Inc., New York, 1998.

[148] R. de Levie, Electrochim. Acta 9 (1964) 1231.

[149] R. de Levie, Electrochim. Acta 8 (1963) 751.

[150] D.P. Almond, C.R. Bowen, D.A.S. Rees, J. Phys. D. Appl. Phys. 39 (2006) 1295.

[151] B. Vianas, D.P. Almond, J. Luo, R. Stevens, Solid State Ionics 126 (1999) 65.

[152] J. Valsa, J. Vlach, Int. J. Circuit Theory Appl. DOI: 10.10 (2011). 
[153] A. Goncharenko, V. Lozovski, E. Venger, Opt. Commun. 174 (2000) 19.

[154] V.-T. Truong, J.G. Ternan, Polymer (Guildf). 36 (1995) 905.

[155] J.-B. Jorcin, M.E. Orazem, N. Pebere, B. Tribollet, Electrochim. Acta 51 (2006) 1473.

[156] D.S. Chambers, D.J. Wheeler, Understanding Statistical Process Control , 2nd ed., SPC Press, Knoxville, TN, 1992.

[157] A.D.R. McQuarrie, C.-L. Tsai, Regression and Time Series Model Selection, World Scientific., 1998.

[158] L. Viña, M. Cardona, Phys. Rev. B 29 (1984) 6739.

[159] R.S. Muller, T.I. Kamins, Device Electronics for Integrated Circuits, 2nd ed., John Wiley, New York, 1986.

[160] N.W. Ashcroft, N.D. Mermin, Solid State Physics, Saunders College, Orlando, FL, 1976.

[161] L. Onsager, J. Chem. Phys. 2 (1934) 599.

[162] J.F. Smalley, H.O. Finklea, C.E.D. Chidsey, M.R. Linford, S.E. Creager, J.P. Ferraris, K. Chalfant, T. Zawodzinsk, S.W. Feldberg, M.D. Newton, J. Am. Chem. Soc. 125 (2003) 2004.

[163] R.A. Marcus, J. Chem. Phys. 24 (1956) 979.

[164] R.A. Marcus, J. Chem. Phys. 24 (1956) 966. 
[165] N. Sutin, J. Phys. Chem. 90 (1986) 3465.

[166] H.B. Callen, Thermodynamics, John Wiley Inc., New York, 1960.

[167] R.S. Muller, T.I. Kamins, M. Chan, Device Electronics for Integrated Circuits, 3rd ed., John Wiley and Sons, New York, 2003.

[168] H. Yamada, P.R. Bandaru, Appl. Phys. Lett. 104 (2014) 213901.

[169] M. Hoefer, P.R. Bandaru, Appl. Phys. Lett. 95 (2009) 183108.

[170] M. Hoefer, P.R. Bandaru, J. Electrochem. Soc. 160 (2013) H360.

[171] H. Yamada, P.R. Bandaru, Appl. Phys. Lett. 102 (2013) 173113.

[172] M. Hoefer, P.R. Bandaru, J. Appl. Phys. 108 (2010) 34308.

[173] V. Augustyn, J. Come, M.A. Lowe, J.W. Kim, P.-L. Taberna, S.H. Tolbert, H.D. Abruña, P. Simon, B. Dunn, Nat. Mater. 12 (2013) 518.

[174] E. Peled, J. Electrochem. Soc. (1979) 2047.

[175] J. Guyer, W. Boettinger, J. Warren, G. McFadden, Phys. Rev. E 69 (2004) 021603.

[176] J. Guyer, W. Boettinger, J. Warren, G. McFadden, Phys. Rev. E 69 (2004) 021604.

[177] E. Levi, E. Zinigrad, H. Teller, M.D. Levi, D. Aurbach, E. Mengeritsky, E. Elster, P. Dan, E. Granot, H. Yamin, 144 (1997) 4133. 
[178] M. Park, X. Zhang, M. Chung, G.B. Less, A.M. Sastry, J. Power Sources 195 (2010) 7904.

[179] D. Aurbach, J. Power Sources 89 (2000) 206.

[180] D. Aurbach, E. Zinigrad, Y. Cohen, H. Teller, 148 (2002) 405.

[181] D. Shanmukaraj, S. Grugeon, S. Laruelle, G. Douglade, J.-M. Tarascon, M. Armand, Electrochem. Commun. 12 (2010) 1344.

[182] C.R. Jarvis, M.J. Lain, M.V. Yakovleva, Y. Gao, J. Power Sources 162 (2006) 800.

[183] J. Liu, A. Manthiram, J. Phys. Chem. C 113 (2009) 15073.

[184] M.G. Scott, J. Electrochem. Soc. 145 (1998) 1506.

[185] M. Singh, I. Gur, H.B. Eitouni, N.P. Balsara, Solid Electrolyte Material Manufacturable by Polymer Processing Methods - Energy Innovation Portal, 2009.

[186] J. Li, N.J. Dudney, J. Nanda, C. Liang, ACS Appl. Mater. Interfaces 6 (2014) 10083.

[187] F. Tran, P. Blaha, Phys. Rev. Lett. 102 (2009) 226401.

[188] H. Ibach, H. Luth, Solid-State Physics: An Introduction to Theory and Experiment , Springer-Verlag, Berlin, 1991.

[189] J.D. Jackson, Classical Electrodynamics, John Wiley, New York, 1999.

[190] H. Kroemer, C. Kittel, Thermal Physics, W.H. Freeman and Co., New York, 1980. 
[191] J.H. Davies, The Physics of Low-Dimensional Semiconductors, Cambridge University Press, New York, 1998.

[192] N. Nilius, T.M. Wallis, W. Ho, Science 297 (2002) 1853.

[193] B.G. Streetman, S. Banerjee, Solid State Electronic Devices, 5th ed., Prentice Hall, New Jersey, 2000.

[194] H.B. Callen, T.A. Welton, Phys. Rev. 83 (1951) 34.

[195] J. Bardeen, Phys. Rev. 71 (1947) 717.

[196] W.P. Gomes, D. Vanmaekelbergh, Electrochim. Acta 41 (1996) 967.

[197] K. Gelderman, L. Lee, S.W. Donne, J. Chem. Educ. 84 (2007) 685.

[198] F. Cardon, Physica 57 (1972) 390.

[199] A.C. Ferrari, D.M. Basko, Nat. Nanotechnol. 8 (2013) 235.

[200] A. Das, S. Pisana, B. Chakraborty, S. Piscanec, S.K. Saha, U. V Waghmare, K.S. Novoselov, H.R. Krishnamurthy, A.K. Geim, A.C. Ferrari, A.K. Sood, Nat. Nanotechnol. 3 (2008) 210.

[201] H. Gerischer, J. Electrochem. Soc. 113 (1966) 1174.

[202] M. Grätzel, Inorg. Chem. 44 (2005) 6841.

[203] M. Grätzel, Nature 414 (2001) 338. 
[204] E.A. Ponomarev, L.M. Peter, J. Electroanal. Chem. 396 (1995) 219.

[205] M.D. Stoller, S. Park, Y. Zhu, J. An, R.S. Ruoff, Nano Lett. 8 (2008) 3498.

[206] Y. Zhu, S. Murali, M.D. Stoller, K.J. Ganesh, W. Cai, P.J. Ferreira, A. Pirkle, R.M. Wallace, K.A. Cychosz, M. Thommes, D. Su, E.A. Stach, R.S. Ruoff, Science 332 (2011) 1537.

[207] H. Ji, X. Zhao, Z. Qiao, J. Jung, Y. Zhu, Y. Lu, L.L. Zhang, A.H. MacDonald, R.S. Ruoff, Nat. Commun. 5 (2014) 3317.

[208] K. Kinoshita, Carbon: Electrochemical and Physicochemical Properties, John Wiley \& Sons, Inc., New York, NY, 1988.

[209] E. Uesugi, H. Goto, R. Eguchi, A. Fujiwara, Y. Kubozono, Sci. Rep. 3 (2013) 1595.

[210] B.C. Wood, T. Ogitsu, M. Otani, J. Biener, J. Phys. Chem. C 118 (2014) 4.

[211] J.N. Israelachvili, Intermolecular and Surface Forces,, 3rd ed., Academic Press, San Diego, 2011.

[212] C.E. Banks, T.J. Davies, G.G. Wildgoose, R.G. Compton, Chem. Commun. 7 (2005) 829.

[213] X. Ji, C. Banks, A. Crossley, R. Compton, J. Phys. Chem. 7 (2006) 1337.

[214] C.C.M. Neumann, C. Batchelor-McAuley, C. Downing, R.G. Compton, Chem. - A Eur. J. 17 (2011) 7320. 
[215] J.-P. Randin, E. Yeager, J. Electroanal. Chem. 58 (1975) 313.

[216] Q. Li, C. Batchelor-McAuley, R.G. Compton, J. Phys. Chem. B 114 (2010) 7423.

[217] K. Kobayashi, Phys. Rev. B 48 (1993) 1757.

[218] N.S. Lewis, Annu. Rev. Phys. Chem. 42 (1991) 543.

[219] M.T. McDermott, R.L. McCreery, Langmuir 10 (1994) 4307.

[220] S. Kwon, R. Vidic, E. Borguet, Surf. Sci. 522 (2003) 17.

[221] D.A. Porter, K.E. Easterling, Phase Transformations in Metals and Alloys, Nelson Thornes, Cheltenham, UK, 1992.

[222] L. Li, S. Reich, J. Robertson, Phys. Rev. B 72 (2005) 184109.

[223] F. Banhart, J. Kotakoski, A. V Krasheninnikov, ACS Nano 5 (2011) 26.

[224] R.M. Martin, Electronic Structure: Basic Theory and Practical Methods, Cambridge University Press, Cambridge, UK, 2004.

[225] D.R. Nelson, Defects and Geometry in Condensed Matter Physics, Cambridge University Press, Cambridge, UK, 2002.

[226] J.-C. Charlier, Acc. Chem. Res. 35 (2002) 1063.

[227] Z. Yao, C. Dekker, P. Avouris, Electronic Properties, Junctions, and Defects of Carbon Nanotubes, Springer-Verlag GmbH, 2001. 
[228] O. Zhou, R.M. Fleming, D.W. Murphy, C.H. Chen, R.C. Haddon, A.P. Ramirez, S.H. Glarum, Science (80-. ). 263 (1994) 1744.

[229] S.G. Louie, in:, G.D. M.S. Dresselhaus and P. Avouris (Ed.), Carbon Nanotub. Synth. Struct. Prop. Appl., Springer, New York, 2001, pp. 113-145.

[230] F. Banhart, Reports Prog. Phys. 62 (1999) 1181.

[231] L. Chico, Phys. Rev. B 54 (1996) 2600.

[232] J. Nichols, C.P. Deck, H. Saito, P.R. Bandaru, J. Appl. Phys. 102 (2007) 64306.

[233] Y. Fan Goldsmith, B.R., and Collins, P.G., Nat. Mater. 4 (2005) 906.

[234] S.L. Mielke Troya, D., Zhang, S., Li, J.-L., Xiao, S., Car, R., Ruoff, R.S., Schatz, G.C., and Belytschko, T., Chem. Phys. Lett. 390 (2004) 413.

[235] I.O. Maciel, N. Anderson, M.A. Pimenta, A. Hartschuh, H. Qian, M. Terrones, H. Terrones, J. Campos-Delgado, A.M. Rao, L. Novotny, A. Jorio, Nat. Mater. 7 (2008) 878.

[236] J.A. Robinson, E.S. Snow, S.C. Badescu, T.L. Reinecke, F.K. Perkins, Nanoletters 6 (2006) 1747.

[237] A. V Krasheninnikov, K. Nordlund, M. Sirvio, E. Salonen, Keinonen, Phys. Rev. B Condens. Matter 63 (2001) 245405.

[238] J.-C. Charlier Ebbesen, T.W., Lambin, Ph., Phys. Rev. B 53 (1996) 11108. 
[239] M. Bockrath, W. Liang, D. Bozovic, J.H. Hafner, C.M. Lieber, M. Tinkham, H. Park, Science (80-. ). 291 (2001) 283.

[240] Q. Lu, B. Bhattacharya, Nanotechnology 16 (2005) 555.

[241] M.A. Pimenta, G. Dresselhaus, M.S. Dresselhaus, L.G. Cançado, A. Jorio, R. Saito, Phys. Chem. Chem. Phys. 9 (2007) 1276.

[242] R. Hoffmann, Solids and Surfaces, A Chemist's View of Bonding in Extended Structures, VCH Publishers, New York, 1988.

[243] A.V. Krasheninnikov, P.O. Lehtinen, A.S. Foster, R.M. Nieminen, Chem. Phys. Lett. 418 (2006) 132.

[244] J. Ma, D. Alfè, A. Michaelides, E. Wang, Phys. Rev. B 80 (2009) 033407.

[245] C.-H. Kiang, M. Endo, P.M. Ajayan, G. Dresselhaus, M.S. Dresselhaus, Phys. Rev. Lett. $81(1998) 1869$.

[246] P. Lehtinen, A. Foster, A. Ayuela, A. Krasheninnikov, K. Nordlund, R. Nieminen, Phys. Rev. Lett. 91 (2003) 017202.

[247] H.C. Choi, M. Shim, S. Bangsaruntip, H. Dai, J. Am. Chem. Soc. 124 (2002) 9058.

[248] C. Ni, J. Chattopadhyay, W.E. Billups, P.R. Bandaru, Appl. Phys. Lett. 93 (2008) 243113.

[249] O. V. Yazyev, A. Pasquarello, Phys. Rev. B 82 (2010) 045407. 
[250] A. Krasheninnikov, P. Lehtinen, A. Foster, P. Pyykkö, R. Nieminen, Phys. Rev. Lett. 102 (2009) 126807.

[251] P.M. Ajayan, T.W. Ebbesen, T. Ichihashi, S. Ijima, K. Tanigaki, H. Hiura, Nature 362 (1993) 522.

[252] J.F. AuBuchon, L.-H. Chen, A.I. Gapin, S. Jin, J. Appl. Phys. 97 (2005) 124310.

[253] N. Pierard, A. Fonseca, Z. Konya, I. Willems, G. Van Tendeloo, J. B.Nagy, Chem. Phys. Lett. 335 (2001) 1.

[254] P.R. Bandaru, C. Daraio, K. Yang, A.M. Rao, J. Appl. Phys. 101 (2007) 94307.

[255] W. Wang, K. Yang, J. Gaillard, P.R. Bandaru, A.M. Rao, Adv. Mater. 20 (2008) 179.

[256] M. Zhang, J. Li, Mater. Today 12 (2009) 12.

[257] K.Y.J.G.P.R.B.A.M.R. W. Wang, 20 (2008) 179.

[258] P.R. Bandaru, C. Daraio, S. Jin, a M. Rao, Nat. Mater. 4 (2005) 663.

[259] M. Terrones, F. Banhart, N. Grobert, J.-C. Charlier, H. Terrones, P.M. Ajayan, Phys. Rev. Lett. 89 (2002) 75501.

[260] P.-G. de Gennes, F. Brochard-Wyart, D. Quere, Capillarity and Wetting Phenomena: Drops, Bubbles, Pearls, Waves, Springer, New York, NY, 2002.

[261] R.T. Morrison, R.N. Boyd, Organic Chemistry, Allyn \& Bacon Inc., Boston, 1983. 
[262] J. Dzubiella, J.-P. Hansen, J. Chem. Phys. 122 (2005) 234706.

[263] B.E. Conway, E. Gileadi, M. Dzieciuch, Electrochim. Acta 8 (1963) 143.

[264] G. Schlichthörl, S.Y. Huang, J. Sprague, A.J. Frank, J. Phys. Chem. B 101 (1997) 8141.

[265] B.B. Mandelbrot, The Fractal Geometry of Nature, W.H.Freeman and Co., 1982.

[266] K.S.W. Sing, Pure Appl. Chem. 54 (1982) 2201.

[267] Y. Gogotsi, R.K. Dash, G. Yushin, T. Yildirim, G. Laudisio, J.E. Fischer, J. Am. Chem. Soc. 127 (2005) 16006.

[268] D.H. Everett, J.C. Powl, J. Chem. Soc. Faraday Trans. 172 (1976) 619.

[269] J. Chmiola, G. Yushin, Y. Gogotsi, C. Portet, P. Simon, P.L. Taberna, Science 313 (2006) 1760.

[270] V. Ballenegger, J.-P. Hansen, Europhys. Lett. 63 (2003) 381.

[271] M. Carrillo-Tripp, H. Saint-Martin, I. Ortega-Blake, Phys. Rev. Lett. 93 (2004) 168104.

[272] B. O’Regan, M. Grätzel, Nature 353 (1991) 737.

[273] J. Bisquert, Phys. Chem. Chem. Phys. 5 (2003) 5360.

[274] S. Mathew, A. Yella, P. Gao, R. Humphry-Baker, B.F.E. Curchod, N. Ashari-Astani, I. Tavernelli, U. Rothlisberger, M.K. Nazeeruddin, M. Grätzel, Nat. Chem. 6 (2014) 242. 
[275] A. Hagfeldt, M. Grätzel, Acc. Chem. Res. 33 (2000) 269.

[276] J. Bisquert, D. Cahen, G. Hodes, S. Rühle, A. Zaban, J. Phys. Chem. B 108 (2004) 8106.

[277] L.. Peter, E.. Ponomarev, G. Franco, N.. Shaw, Electrochim. Acta 45 (1999) 549.

[278] L.. Peter, N.. Duffy, R.. Wang, K.G.. Wijayantha, J. Electroanal. Chem. 524-525 (2002) 127.

[279] K. Schwarzburg, R. Ernstorfer, S. Felber, F. Willig, Coord. Chem. Rev. 248 (2004) 1259.

[280] S. Luryi, Appl. Phys. Lett. 52 (1988) 501.

[281] D.L. John, L.C. Castro, D.L. Pulfrey, J. Appl. Phys. 96 (2004) 5180.

[282] T. Fang, A. Konar, H. Xing, D. Jena, Appl. Phys. Lett. 91 (2007) 92109.

[283] J. Xia, F. Chen, J. Li, N. Tao, Nat. Nanotechnol. 4 (2009) 505.

[284] R. Saito, G. Dresselhaus, M.S. Dresselhaus, Phys. Rev. B 61 (2000) 2981.

[285] P.R. Bandaru, J. Nanosci. Nanotechnol. 7 (2007) 1239.

[286] R. Saito, M. Fujita, G. Dresselhaus, M.S. Dresselhaus, Appl. Phys. Lett. 60 (1992) 2204.

[287] C. Beenakker, Rev. Mod. Phys. 80 (2008) 1337.

[288] C. Klein, W. Straub, Phys. Rev. 123 (1961) 1581.

[289] D.K. Efetov, P. Kim, Phys. Rev. Lett. 105 (2010) 256805. 
[290] J.D. Wiggins-Camacho, K.J. Stevenson, J. Phys. Chem. C 113 (2009) 19082.

[291] F. Tuinstra, J.L. Koenig, J. Chem. Phys. 53 (1970) 1126.

[292] D.S. Knight, W.B. White, J. Mater. Res. 4 (1989) 385.

[293] B. Conway, Electrochemical Supercapacitors: Scientific Fundamentals and Technological Applications, Kluwer Academic, New York, 1999.

[294] V. Augustyn, P. Simon, B. Dunn, Energy Environ. Sci. 7 (2014) 1597.

[295] M.V. Sudha, V. SANGARANARAYANAN, J. Chem. Sci. 117 (2005) 207.

[296] S. Hadżi-Jordanov, H. Angerstein-Kozlowska, M. Vukovič, B.E. Conway, J. Electrochem. Soc. 125 (1978) 1471.

[297] P. Stonehart, H.A. Kozlowska, B.E. Conway, Proc. R. Soc. A Math. Phys. Eng. Sci. 310 (1969) 541.

[298] H. Angerstein-Kozlowska, J. Klinger, B.E. Conway, J. Electroanal. Chem. Interfacial Electrochem. 75 (1977) 61.

[299] H. Angerstein-Kozlowska, J. Klinger, B.E. Conway, J. Electroanal. Chem. Interfacial Electrochem. 75 (1977) 45.

[300] B.E. Conway, Prog. Surf. Sci. 16 (1984) 1.

[301] H.B. Callen, Thermodynamics, John Wiley Inc., New York, 1960. 
[302] J.H. De Boer, Adv. Catal. 8 (1956) 17.

[303] J.G.N. Thomas, Trans. Faraday Soc. 57 (1961) 1603.

[304] B.E. Conway, E. Gileadi, Trans. Faraday Soc. 58 (1962) 2493.

[305] I. Higuchi, T. Ree, H. Eyring, J. Am. Chem. Soc. 77 (1955) 4969.

[306] R. Huggins, Solid State Ionics 134 (2000) 179.

[307] L. Mai, H. Li, Y. Zhao, L. Xu, X. Xu, Y. Luo, Z. Zhang, W. Ke, C. Niu, Q. Zhang, Sci. Rep. 3 (2013) 1718.

[308] A. Mendiboure, C. Delmas, P. Hagenmuller, J. Solid State Chem. 57 (1985) 323.

[309] S. Ardizzone, G. Fregonara, S. Trasatti, Electrochim. Acta 35 (1990) 263.

[310] H. Vogt, Electrochim. Acta 39 (1994) 1981.

[311] D. Baronetto, N. Krstajic, S. Trasatti, Electrochim. Acta 16 (1994) 2359.

[312] J.P. Zheng, P.J. Cygan, T.R. Jow, J. Electrochem. Soc. 142 (1995) 2699.

[313] S.T. Senthilkumar, R.K. Selvan, N. Ponpandian, J.S. Melo, Y.S. Lee, J. Mater. Chem. A 1 (2013) 7913.

[314] G. Lota, K. Fic, E. Frackowiak, Electrochem. Commun. 13 (2011) 38. 
[315] S. Roldán, Z. González, C. Blanco, M. Granda, R. Menéndez, R. Santamaría, Electrochim. Acta 56 (2011) 3401.

[316] M. Meller, J. Menzel, K. Fic, D. Gastol, E. Frackowiak, Solid State Ionics 265 (2014) 61.

[317] E. Frackowiak, K. Fic, M. Meller, G. Lota, ChemSusChem 5 (2012) 1181.

[318] E.O. Barnes, X. Chen, P. Li, R.G. Compton, J. Electroanal. Chem. 720-721 (2014) 92.

[319] G. Keeley, M. Lyons, Int J Electrochem Sci 4 (2009) 794.

[320] M.C. Henstridge, E.J.F. Dickinson, M. Aslanoglu, C. Batchelor-McAuley, R.G. Compton, Sensors Actuators B Chem. 145 (2010) 417.

[321] K.R. Ward, L. Xiong, N.S. Lawrence, R.S. Hartshorne, R.G. Compton, J. Electroanal. Chem. 702 (2013) 15.

[322] A.T. Hubbard, F.C. Anson, in:, Electroanalaytical Chem. A Ser. Adv., 1970, pp. 129-214.

[323] J. Weidner, P. Fedkiw, J. Electrochem. Soc. 138 (1991) 2514.

[324] N.R. De Tacconi, A.J. Calandra, A.J. Arvía, Electrochim. Acta 18 (1973) 571.

[325] S.-B. Yoon, H.-K. Song, K.C. Roh, K.-B. Kim, J. Electrochem. Soc. 161 (2013) A137.

[326] E. Laviron, J. Electroanal. Chem 52 (1974) 355.

[327] C. Guan, X. Li, Z. Wang, X. Cao, C. Soci, H. Zhang, H.J. Fan, Adv. Mater. 24 (2012) 4186. 
[328] C. Zhou, Y. Zhang, Y. Li, J. Liu, Nano Lett. 13 (2013) 2078.

[329] L. Huang, D. Chen, Y. Ding, S. Feng, Z.L. Wang, M. Liu, Nano Lett. 13 (2013) 3135.

[330] X. Xia, J. Tu, Y. Zhang, X. Wang, C. Gu, X.-B. Zhao, H.J. Fan, ACS Nano 6 (2012) 5531

[331] L. Wan, J. Xiao, F. Xiao, S. Wang, ACS Appl. Mater. Interfaces 6 (2014) 7735.

[332] G. Lota, E. Frackowiak, Electrochem. Commun. 11 (2009) 87.

[333] J.A. Irvine, D.J. Irvin, J.D. Stenger-Smith, Handbook of Conducting Polymers, 3rd ed., Taylor \& Francis , Boca Raton, FL, 2007.

[334] C. Arbizzani, M. Mastragostino, L. Meneghello, Electrochim. Acta 41 (1996) 21.

[335] M. Mastragostino, C. Arbizzani, F. Soavi, Solid State Ionics 148 (2002) 393.

[336] S. Ghosh, O. Inganäs, Adv. Mater. 11 (1999) 1214.

[337] G. Milczarek, O. Inganäs, Science 335 (2012) 1468.

[338] K. Zhang, B. Tieke, J.C. Forgie, F. Vilela, J.A. Parkinson, P.J. Skabara, Polym. (in Press. (2010).

[339] P.J. Skabara, R. Berridge, E.J.L. McInnes, D.P. West, S.J. Coles, M.B. Hursthouse, K. Mullen, J. Mater. Chem. 14 (2004) 1964. 
[340] R. Berridge, P.J. Skabara, C. Pozo-Gonzalo, A. Kanibolotsky, J. Lohr, J.J.W. McDouall, E.J.L. McInnes, J. Wolowska, C. Winder, N.S. Sariciftci, R.W. Harrington, W. Clegg, J. Phys. Chem. B 110 (2006) 3140.

[341] G. Milczarek, O. Inganäs, Science (80-. ). 335 (2012) 1468.

[342] "Wooden batteries", Economist (2012).

[343] G. Milczarek, Langmuir 25 (2009) 10345.

[344] G. Milczarek, Electroanalysis 19 (2007) 1411.

[345] D.A.. Rand, D.. Boden, C.. Lakshmi, R.. Nelson, R.. Prengaman, J. Power Sources 107 (2002) 280 .

[346] C. Liu, A.J. Bard, Nat. Mater. 7 (2008) 505.

[347] C. Liu, A.J. Bard, J. Am. Chem. Soc. 131 (2009) 6397.

[348] H.T. Baytekin, A.Z. Patashinski, M. Branicki, B. Baytekin, S. Soh, B.A. Grzybowski, Science 333 (2011) 308.

[349] H.T. Baytekin, B. Baytekin, T.M. Hermans, B. Kowalczyk, B.A. Grzybowski, Science $341(2013) 1368$.

[350] L. Shao, J.-W. Jeon, J.L. Lutkenhaus, J. Mater. Chem. A 1 (2013) 7648.

[351] J.W. Long, B. Dunn, D.R. Rolison, H.S. White, Chem. Rev. 104 (2004) 4463. 
[352] T.S. Arthur, D.J. Bates, N. Cirigliano, D.C. Johnson, P. Malati, J.M. Mosby, E. Perre, M.T. Rawls, A.L. Prieto, B. Dunn, MRS Bull. 36 (2011) 523.

[353] B. Dunn, J.W. Long, D.R. Rollison, Electrochem. Soc. Interface Fall (2008) 49.

[354] V. Zadin, H. Kasemägi, A. Aabloo, D. Brandell, J. Power Sources 195 (2010) 6218.

[355] J. Souquet, Solid State Ionics 148 (2002) 375.

[356] A. Aboulaich, R. Bouchet, G. Delaizir, V. Seznec, L. Tortet, M. Morcrette, P. Rozier, J.M. Tarascon, V. Viallet, M. Dollé, Adv. Energy Mater. 1 (2011) 179.

[357] G. Delaizir, V. Viallet, A. Aboulaich, R. Bouchet, L. Tortet, V. Seznec, M. Morcrette, J.M. Tarascon, P. Rozier, M. Dollé, Adv. Funct. Mater. 22 (2012) 1993.

[358] G. Oltean, H. Desta Asfaw, L. Nyholm, K. Edstrom, ECS Electrochem. Lett. 3 (2014) A54. 


\section{Figure Captions}

Figure 1 (from Ref. [40]) A diagram depicting the energy levels of the anode (a supplier of electrons) and cathode (an acceptor of electrons) electrodes with respect to the electrolyte energy levels (i.e., the LUMO and the HOMO). As electron transfer occurs from a higher energy level to a lower energy level, proper positioning of the energies of the constituent elements in a battery would be necessary to prevent charge transfer into the electrolyte when the battery is not connected to an external circuit. Then, all the electrons would need to traverse the external circuit from the higher energy level anode to the lower energy cathode. 
Figure 2 (from Ref. [35]). The discharge curve of a battery exhibiting the loss of the thermodynamically predicted open circuit voltage $\left(\mathrm{OCV} / V_{O C}\right)$ due to various charge transfer mechanisms between the electrode and electrolyte, leading to a higher resistance.

Figure 3 (from Ref. [38]) The operating principle of a Li ion battery involves the movement of $\mathrm{Li}^{+}$ions within the electrolyte to/from the electrodes. The above diagram shows the use (through charge and discharge) of the battery, where electrons move in the external circuit from the anode (negative electrode) to the cathode (positive electrode) and the $\mathrm{Li}^{+}$ions move in the electrolyte (another $\mathrm{Li}$ compound with high ionic conductivity) to compensate for the received charge.

Figure 4 (from Ref. [40]) The insertion of $\mathrm{Li}^{+}$ions into tetrahedral or octahedral sites in spinel structures (say, $\mathrm{Mn}_{3} \mathrm{O}_{4}$ ) may cause redox couples of different energies to be activated, e.g., $\mathrm{Mn}^{3+} / \mathrm{Mn}^{4+}$ states for the octahedral states and $\mathrm{Mn}^{2+} / \mathrm{Mn}^{3+}$ states for the tetrahedral states. The energy difference is manifested as a step in the observed potential.

Figure 5 (from Ref. [40]) A schematic of the energy configuration of the redox states, relevant for $\mathrm{Li}$ ion batteries, reckoned with respect to the $\mathrm{Li} / \mathrm{Li}^{+}$potential. The figure on the far left indicates the anode configuration with potentials corresponding to $\mu_{A}-$ see Figure 1, while the other figures schematics represent cathode materials, corresponding 
to $\mu_{C}-$ see Figure 1. It is indicated that (a) the voltage limits $\left(=\mu_{A} \square \square \mu_{C}\right)$ are greater for oxides compared to the sulfides, (b) enhanced redox potential of Co with respect to $\mathrm{Ti},(\mathrm{c})$ polyanions (e.g., $\mathrm{PO}_{4}^{-}$), replacing the oxide ions can enhance binding energy and the $V_{O C}$.

Figure 6 (from Ref. [40]) The interaction of the redox couple energy level $\left(=E_{F}\right)-$ constituted from the transition metal cations and which may be manipulated through charge transfer, with the anion bands. In (a) the carriers involved in charge transfer, mostly holes, are itinerant and independent, while in (b) the level interaction splits the redox level into anti-bonding (a.b.) states, which can accommodate holes and are derived from the anions and bonding $(b)$ states derived from the cations, where the dominant carriers are electrons. (c) When the transition states are too far down in the anionic band, the electrons are the majority carriers.

Figure 7 An electrochemical capacitor (EC) stores charge and energy (a) capacitively through an electrical double layer formed across a charged electrode, and (2) electrochemically, due to redox reactions occurring at the electrode surface. The stored energy density $(W)$ is proportional to the net capacitance due to these mechanisms, over a voltage range $(V)$, and is given per unit mass $(m)$ as $W=C V^{2} / 2 m$, while the power density $(P=W / \tau)$, is related to the charge/discharge times, $\tau(=R C)$, where $R$ refers to the net resistance of the electrochemical system. 
Figure 8 (a) The formation of a double layer constituted from an increased concentration of oppositely charged ions on a positive electrode is shown. The spatial extent of the Helmholtz layer is dependent on the character of ions (cations and anions, the degree if solvation), (b) The two capacitances $C_{H}$ and $C_{D}$ model the immobile charges right next to the electrode and the mobile charges further away in the electrolyte, (c) The distribution of the mobile charges in the metal electrode (on the left) and the electrolyte (on the right), (d) Much of the applied voltage on the electrode is dropped across the immobile Helmholtz layer.

Figure 9 (a) A triangular voltage/potential (of magnitude $E_{\square}$ ) waveform applied over a time $t_{\lambda}$ for cyclic voltammetry induces both anodic and cathodic currents (b) A typical voltammogram indicating cathodic reduction (and the corresponding current: $i_{p, c}$ as well as the anodic oxidation, with the corresponding current: $i_{p, a}$ for a one electron transfer reaction. The $i_{b}$ and $i_{l i m}$ are the baseline and limiting currents, respectively.

Figure 10 (from Ref. [126]) A Current-Voltage profile of an ideal capacitor. Such a curve is obtained in the absence of Faradic reactions (no peak currents: $i_{P}$ as in Figure 9(b). The steady state operation characteristics of a double-layer capacitance are indicated in the figure, i.e., the transient development of a current in response to a voltage increase from zero is not shown. 
Figure $11 \mathrm{CV}$ illustrating irreversible electron transfer and adsorption of the redox couple during the anodic sweep, adapted from Ref.[122]

Figure 12 (from Refs. [70] and [102]), (a) $\Psi(E)$ as a function of instantaneous potential, E. Dashed lines indicate reversibility and the Roman numerals represent different scan rates, where I, II, III, and IV correspond to $\Lambda \square=10,1,0.1$, and 0.01 , respectively. (b) $\Xi(\Lambda, \alpha)$ as a function of $\log (\Lambda)$ for different values of $\alpha \square \square$ Dashed lines indicate total irreversibility.

Figure 13 (from Ref. [135]) Variation of the peak separation as a function of the scan rate $(s)$. The $m=(R T / F) k_{s} / n s$, where $k_{s}$ is the electrochemical rate constant and $n$ is the number of transferred electrons in the redox reaction.

Figure 14 (a) A square current waveform is used for cyclic constant current chronopotentiometry. The current is cycled between a positive $\left(i_{o}\right)$ and a negative value $\left(-i_{o}\right)$. (b) The resulting voltage response to current waveform for an ideally polarizable capacitor in series with a resistor. The equivalent series resistance (ESR) may be estimated by $R_{E S R}=\Delta V / 2 i_{o}$. 
Figure 15 The equivalent circuit of an electrochemical systems may be constituted from a double-layer capacitance: $C_{d l}$ in addition to a solution resistance: $R_{s}$, a charge-transfer resistance: $R_{c t}$, and a Warburg impedance: $Z_{w}$ to model diffusional constraints. The Faradaic impedance is depicted through the latter two components.

Figure 16 The Nyquist plots (of the imaginary: $Z_{\text {imag, } R}$ and the real: $Z_{\text {real }, R}$ components of the net impedance) of the Randles circuit for the cases of (a) $\omega \rightarrow \infty$, and (b) $\omega \rightarrow 0$. Note that the frequency increases in a counter-clockwise sense or from right to left.

Figure 17 (a) A Nyquist plot indicating the "knee frequency", which denotes the transition from a kinetically dominated regime (characteristic of capacitors) to a diffusion process dominated regime (characteristic of batteries). (b) A plot where $Z_{w}$ is replaced by a Faradaic capacitance: $C_{F}$ but remains in series with $R_{c t}$ (see Figure 15) to demonstrate a transition to ideal capacitor behavior.

Figure 18 (from Ref. [142]) The phenomenon of self-discharge is thermodynamically driven and is relevant to the conversion of a high energy charged state to a lower energy charge state. Such characteristics determine the shelf life of practical electrochemical capacitors and batteries. 
Figure 19 (from Ref. [142]) A model for the self-discharge in an electrochemical capacitor, indicating Faradaic $\left(R_{F}\right)$ or leakage resistances across the left $(1)$ and right (2) electrode double layer capacitance $\left(C_{d l}\right)$. The series resistances due to the electrolyte $\left(R_{e}\right)$ and the separator $\left(R_{s}\right)$ are also shown. Such a model allows for asymmetric discharge at one or both the electrodes.

Figure 20 (from Ref. [148]) The distributed resistance and capacitance model for the analysis of a pore, e.g., in activated carbon or in ordered nanotube arrays.

Figure 21 A possible impedance model consisting of additional resistance and capacitance contributions ( $c f$. in addition to those indicated in Figure 15). In non-metallic materials, the space charge capacitance: $C_{s c}$ (see Section 2.3.2), a quantum capacitance: $C_{Q}$ (see Section 4.11) in addition to a Faradaic/surface area dependent pseudocapacitance: $C_{p}$ (see Section 5.1) as well as concomitant leakage/shunt resistances: $R_{s h}$ may be present.

Figure 22 Circuit models for (a) an equivalent series resistance (ESR) model, and (b) a twodimensional random network of resistors and capacitors (2D RC) model, may be used to fit the $Z(\omega)$ variation. The ratio of the number of capacitors to the total number of components, corresponds to the $\Theta$ of Eqn. 34. 
Figure 23 A schematic free energy $(G)$ - reaction coordinate $(\mathrm{q})$ diagrams for a single electron redox reaction of the form: $O+e^{-} \rightarrow R,(z=1$ in Eqn. 2), where $O$ and $R$ represent the oxidized and reduced species, respectively. The curves for the $O$ and $R$ are displaced in $\mathbf{q}$, from the assumption that the latter moiety is larger than the $O$ species. The E.A. (electron affinity) of the $O$ and the I.E. (ionization energy) relative to the $R$ is indicated. The vertical offset of the $O$ and $R$ curves are indicative of their reorganization energies $(\lambda)$.

Figure 24 (from Ref. [81]) Schematic of the energy transfer from a metal electrode to an electrolyte. The vertical axis represents the energy levels of the metal (far left) and the electrolyte (middle) and the charge transfer is a function of the number of occupied and vacant levels (as represented through the density of states: $\mathrm{D}(\mathrm{E})$, for the metal and $\mathrm{W}(\mathrm{E})$, for the electrolyte). At equilibrium the Fermi energy of the metal electrode $\left(E_{F, \text { metal }}^{0}\right)$ coincides with the energy of the redox couple $\left(E_{F, \text { redox }}^{0}\right)$ resulting in an exchange current, which may be augmented (i.e., $\mathrm{i}^{-}$or $\mathrm{i}^{+}$by an external negative or positive bias voltage/overpotential, respectively).

Figure 25 (from Ref. [81]) Quantum mechanical tunneling processes are involved in electron transfer from (/to) the electrode to (/from) the electrolyte.

Figure 26 (from Ref. [168]) (a) Schematic representation of the charge density $\rho$, with electrode charge $Q_{m}$ and electrolyte ion $Q_{i o n} . L$ is the total device length. (b) The 
electric field $\xi$ variation, depicting the field in in the double-layer: $\xi_{d l}$ and in the electrolyte: $\xi_{e l} . \xi$ is symmetric, and $L^{\prime}$ is the length excluding the double-layers. (c) The electric potential $V$, with double-layer potential $2 V_{d l}$ and electrolyte potential $V_{e l}$. The counter electrode is assigned a reference potential of zero.

Figure 27 (from Ref. [168]) The comparison of voltage profiles at two different times, $t$ and at $t+\Delta t$, from which it can be seen that the increase in $V_{a p p}$ is always greater than that for $V_{e l}$. In general, a faster scan rate allows for a larger value of $\gamma$ and thus $V_{e l}$ due to weaker screening.

Figure 28 (from Ref. [168]) Current density $J$ as a function of the product of the $\mathrm{K}_{3} \mathrm{Fe}(\mathrm{CN})_{6}$ molar concentration and the square root of scan rate for $\mathrm{Pt}$ and carbon nanotube (CNT) nanostructure constituted electrode, are compared to the drift model of Eqn. (42).

Figure 29 (from Ref. [18]) A typical energy band diagram of metal (on the right) semiconductor (on the left) contact under thermal equilibrium, indicating the parameter of interest when evaluating contact characteristics. $\phi_{m}$, the work function of the metal electrode, $(\chi)$ is the material relevant electron affinity, $\left(\Delta \phi_{B n} \square\right)$ the upward band bending of the conduction band: $V_{B O}$, and the energy barrier for motion of the electrons to/fro the material, $\left(\Delta \phi_{n} \square \square \square\right.$ is the electric field induced barrier lowering, $\Delta_{o}$ is voltage drop across the contact-material charged double-layer and $\phi_{o}$ indicates the surface state 
filling. $\left(Q_{S S}\right)$ is the net surface state charge density and $\left(Q_{S C}\right)$ is the charge in the depletion layer of the semiconductor and thus $Q s S$ and $Q s c$ constitute the total charge present in the material. $Q_{m}\left(=-\left[Q_{S S}+Q_{S C}\right]\right)$, represents an equal and opposite charge; this is induced in the metal, considering a very small interfacial layer of thickness ( $\delta \square \square$ and efficient charge transfer or unity carrier transmission probability.

Figure 30 (from Ref.[81]) The interface between an n-type semiconductor and an electrolyte could involve electron transfer through either the $\mathrm{CB}$ or the VB. In (a) the $E_{F, \text { redox }}^{0}$ is closer to the $\mathrm{CB}$ resulting in a greater cathodic current, while in (b) the $E_{F, \text { redox }}^{0}$ is closer to the VB resulting in a greater anodic current.

Figure 31 (from Ref. [203]) The coupling of external illumination to electrochemical processes in a photo-electrochemical cell. (a) Incident light of energy greater than the semiconductor bandgap, say, from the sun, can excite electron-hole pairs which induces redox reactions in the electrolyte. The gain of holes is compensated through the electrons from the counter electrode. (b) Alternately, photosynthetic cells[203] operate through two redox systems, through oxidation (/reduction) of the water at the left (/right) electrode to $\mathrm{O}_{2}\left(/ \mathrm{H}_{2}\right)$. 
Figure 32 (from Ref. [223]) Jahn-Teller distortion as a result of a single vacancy results in a deviation from the periodic hexagonal arrangement in a structure with a graphene motif (e.g., carbon nanotubes) yielding non-hexagonal units.

Figure 33 (a) A Stone-Wales (S-W) defect, yielding non-hexagonal rings, is unique to graphitic systems and occurs through a $\mathrm{C}-\mathrm{C}$ bond rotation, (b) Bond rotation energy as a function of bond angle for graphene - from Ref. [222], indicating that the most favorable angle of rotation s $90^{\circ}$, (c) A practical manifestation of a bent CNT which may exhibit defects both at the lattice and the macroscopic level.

Figure 34 (from Ref. [247]) Au and Pt clusters on a CNT and the associated energies of the metals compared to the Fermi energy of the CNT.

Figure 35 (Adapted from Ref. [223] ) Various edge plane configurations with edge atoms in red: (a) armchair, (b) reconstructed armchair, (c) zigzag, (d) reconstructed zigzag.

Figure 36 Defect induced morphology in carbon nanotubes- from Refs. [254]-[256], e.g., (a) Hollow CNT (HCNT), (b) Y-Junction HCNT[257], (c) Coiled HCNT, (d) Bamboo type CNT, and (e) Herringbone type CNT. 
Figure 37 (from Ref. [125]) Illustrations of delocalized $\pi$-bonding in (a) pristine, (b) boron (B-) doped, and (c) nitrogen (N-) doped graphitic structures.

Figure 38 (from Ref. [125]) Schematic of the local density of states (LDOS) of (a) a boron doped SWCNT and a (b) nitrogen doped SWCNT.

Figure 39 (from Ref. [268]) (A) An increase in the capacitance (normalized to the BET methodology determined surface area) below a pore size of $\sim 1 \mathrm{~nm}$ was observed in TiC-CDC (carbide derived carbons), and ascribed to solvation shell breakdown effects. (B), (C) and (D) indicate the respective regimes (I), (II) and (III) in the graph. In (I), the capacitance is dictated mostly by conventional double layer capacitance. As the pore size decreases, say in (II), the overlap of the double layers results in a further decrease of the capacitance. On further pore size decrease to values corresponding to the electrolyte ion size, it was thought that the distortion of the solvation shells could render the parent ions closer to the electrode and further increase the capacitance, as indicated in Region (III).

Figure 40 (taken from Ref. [271]) A schematic of the working of a dye-sensitized solar cell (DSSC). A wide bandgap semiconductor (e.g., $\mathrm{TiO}_{2}$ ), placed on a transparent conductive oxide electrode, is covered with a photosensitive dye. Illumination causes the excitation of electron-hole pairs in the dye. Charge separation, constituting the electrical current in the external circuit is achieved through the transfer of electrons to 
the $\mathrm{TiO}_{2}$ and the holes to the electrolyte, which undergoes a redox reaction. The potential difference $(\Delta V)$ between the quasi-Fermi level in the $\mathrm{TiO}_{2}$ electrode and the electrochemical potential of the electrolyte corresponds to the cell voltage.

Figure 41 (from Ref. [272]) The components of the capacitance of an ensemble of nanostructured particles consists, at a positive electrode bias, of (A) the conventional Helmholtz capacitance $\left(C_{H}\right)$ : - see Section 2.3.1, between the electrode and the electrolyte. At increasingly negative potentials, electron transfer to the nanoparticles (e.g., $\mathrm{TiO}_{2}$ in a DSSC) enhances their chemical potential and yields a voltage dependent chemical capacitance - (B). At even larger negative potentials, the capacitance of the $\mathrm{TiO}_{2}$ ensemble - electrolyte interface (a form of chemical capacitance: $C_{c h e m}$, as discussed in Section 4.10) dominates and is larger than (A) and (B) due to the increased interfacial area.

Figure 42 (from Ref. [171]) (a) Representation of nanostructure (e.g., CNT) DOS and electrolyte DOS (as columns) along with their respective Fermi energies $\left(E_{F}\right)$ at equilibrium, with no applied voltage $(\Delta \mathrm{V}=0)$ - top figure, and with a non-zero applied voltage $(\Delta \mathrm{V} \neq 0)$ bottom figure. The applied $\Delta \mathrm{V}$ causes a differential change in the $E_{F}\left(\right.$ of $\left.\Delta E_{F}\right)$ and is partitioned between the CNT electrode (as $\Delta \mathrm{V}_{\mathrm{Q}}$ ) and the bulk electrolyte $\left(\Delta \mathrm{V}_{\mathrm{E}}\right)$. While $\Delta \mathrm{V}_{\mathrm{Q}}$ would be associated with the $C_{Q}$, the $\Delta \mathrm{V}_{\mathrm{E}}$ is related to the $C_{d l}$.

(b) The quantum capacitance $\left(C_{Q}\right)$ as a function of $E_{F}$ in a multi-walled CNT with 15 walls. The initially flat $C_{Q}$ is caused by the metallic CNT walls. The staircase like structure arises from the contributions of successive sub-bands to the DOS. 
Figure 43 (from Ref. [171]) A schematic of electrode configuration in an electrochemical capacitor, zoomed into a section of the CNT array. $C_{Q}$ and $C_{d l}$ in series represented within a single CNT. As the surface area for the CNT electrode (in red) is much higher than that of the counter electrode (in green), the capacitance of the former is much more significant.

Figure 44 (from Ref. [171]) Comparison of experimentally measured capacitance (black squares) with numerical estimates (red lines) of $C_{Q}$, as a function of electrolyte concentration, $I$. A $C_{Q}$ of $20 \mu \mathrm{F} / \mathrm{cm}^{2}$ corresponds to the theoretically predicted $C_{Q}$ of $13 \mathrm{fF} / \mu \mathrm{m}$. The agreement is strongest for low $I$ with low $C_{Q}$ and for high $I$ with high $C_{Q}$.

Figure 45 (from Ref. [293]) Pseudocapacitive (PC) phenomena in materials may be used for devices intermediate to the high energy density of batteries and the high power densities of conventional ECs (Section 2.3). In terms of the latter, time scales between 10 s- 10 minutes have been suggested for PC based devices.

Figure 46 (from Ref. [295]) A cyclic voltammogram of $\mathrm{Ru}$ electrodes in a $\mathrm{H}_{2} \mathrm{SO}_{4}$ electrolyte, indicates reversibility over a range of voltages due to the gradual, surface area dependent, oxidation of the Ru. Such profiles are observed on repeated cycling and oxidation of the Ru surface to a relatively stable oxide. The surface conditioning then yields an area dependent reduction/oxidation of the formed oxide. While the envelope of the curve mimics the rectangular shape of a double layer capacitor - see Figure 10, 
there is a distinct difference in the underlying mechanisms characteristic of pseudocapacitance.

Figure 47 (from Ref. [142]) Successive charge and discharge cycles in (a) thick and (b) thin films of $\mathrm{RuO}_{2}$ (conducted in $0.5 \mathrm{M} \mathrm{H}_{2} \mathrm{SO}_{4}$ at $298 \mathrm{~K}$ ) between $0.7 \mathrm{~V}$ and $0.02 \mathrm{~V}$. There was an easier access to the re-distribution of the oxidation state in the latter and the charge/discharge could be modeled through diffusional processes.

Figure 48 (from Ref. [298]) Cyclic voltammograms indicating a peaked pseudocapacitance. While a low scan rate $(s) /$ net chemical equilibrium rate constant $(k)$ ratio indicates reversibility, increasing values of the ratio indicate a transition to irreversibility and battery-like behavior - also see Figure 52.

Figure 49 (from Ref. [123]) A plot of the surface coverage, $\boldsymbol{\theta}$ and the $C_{P C}$ with respect to the electrode potential, $V$ in accordance with Eqns. (50) and (51). The coverage increases monotonically with the potential while the $C_{P C}$ exhibits a peaked shape characteristic of the $\boldsymbol{\theta}(1-\boldsymbol{\theta})$ variation. Mutual interactions between the adsorbates on the surface are considered through the " $g$ " parameter - a larger $g$ implies lower adsorbate-electrode interactions with the consequence that the coverage occurs over a larger range of electrode potentials. There is a gradual rearrangement of the surface layers towards a two-dimensional ordered configuration. 
Figure 50 (from Ref. [298]) The capacitance $(C)$ - voltage $(V)$ profiles as a function of the $g$ parameter, for (a) reversible, and (b) irreversible conditions[297]. The scan rate/chemical rate constant ratio, $s / k$, is used as a measure of the reversibility, as in Figure 48(d).

Figure 51 (from Ref. [262]) The pseudocapacitance, at a given potential, is composed of a series combination of a coverage dependent Langmuir capacitance as well as an adsorbate interaction dependent Temkin capacitance. A flatness of the capacitancevoltage profile is basically obtained through a consideration of the latter term, through " $r$ ": see Eqn. (56), which is the coefficient of reduction of the adsorbate energy/heats of adsorption[262]. The asymmetry in the profiles seems to be a specific function of the adsorbate interactions, e.g., $\square$ when dipole repulsions are important $(n=1.5)$ or when repulsions in the ionic adsorbed layer are important (with $n=0.5$ ). The " $n$ " in the graph corresponds to the definition of Eqn. (56).

Figure 52 (from Ref. [126]) Cyclic voltammogram of a $\mathrm{Pb} / \mathrm{PbCl}_{2}$ battery material indicating the expected irreversible characteristics between the anodic and the cathodic scans with a wide variety of scan rates (numbers listed on the individual scans). Such irreversibility arises due to the phase changes involved in battery behavior at specific reduction and oxidation voltages. Such a diagram is to be compared with Figure 48(d) at large scan rate/chemical rate constant ratios, where phase changes are likely absent. 
Figure 53 (from Ref. [173] ) (a) Intercalation of $\mathrm{Li}^{+}$ions into the $\mathrm{Nb}_{2} \mathrm{O}_{5}$ structure (with $\mathrm{Nb}$ atoms inside the polyhedra and $\mathrm{O}$ - red atoms) and subsequent reaction of the form: $\mathrm{Nb}_{2} \mathrm{O}_{5}+x \mathrm{Li}^{+}+x \mathrm{e}^{-} \leftrightarrow$ LixNb2O5 (with $x=2$ )was proposed as a new form of PC, (b) Plot of the charge capacity vs. $1 / \sqrt{s}$ indicates a linear relationship at high scan rates (> $20 \mathrm{mV} / \mathrm{s}$ ) indicating a diffusion limited regime and a relatively constant value at lower scan rates indicting capacitive behavior, (c) The rate capability of a $\mathrm{Nb}_{2} \mathrm{O}_{5}$ electrode, based on intercalation PC, can exceed that of a traditional battery anode material (i.e., $\mathrm{Li}_{4} \mathrm{Ti}_{5} \mathrm{O}_{12}$ ) due to the linear dependence of the current on the scan rate in the former.

Figure 54 Electrochemical kinetics in macroporous electrodes constituted from nanotube arrays. (a) Diffusion layer contours overlap as the voltage scan rate $(s)$ decreases, i.e., $s_{3}>s_{2}>s_{1}$, resulting in planar diffusion to the top of the electrode. (b) Inside the pores, a decreased concentration gradient results in thin layer behavior, at lower scan rates, and more of the electrolyte volume can be accessed.

Figure 55 (a) Cyclic Voltammograms (CV) depicting increasing peak currents with $s$, (b) Peak currents due to the combined contributions from the thin layer current and the planar diffusion current (red), (c) effect of Ohmic distortion (red) due to the solution resistance on the currents observed in the $\mathrm{CV}$. 
Figure 56 (a) Galvanostatic discharge curves (at $200 \mathrm{~mA} / \mathrm{cm}^{2}$ ) for various $\mathrm{K}_{3} \mathrm{Fe}(\mathrm{CN})_{6}$ concentrations, inset: shows that the Faradaic capacity varies linearly with the concentration, (b) The capacity, normalized to the nominal thin layer capacity, as a function of discharge current density. It was seen that the capacity contribution from diffusion processes is negligible beyond $50 \mathrm{~mA} / \mathrm{cm}^{2}$ and mostly is due to thin layer attributes at $200 \mathrm{~mA} / \mathrm{cm}^{2}$.

Figure 57 (from Ref. [340]) CV plot of a lignin-polypyrrole composite electrode in 0.1 $\mathrm{M} \mathrm{H}_{2} \mathrm{SO}_{4}$ indicates (A) a broad rectangular profile typical of double layer capacitance see Figure 10, from the background Ppy matrix, and (B) sharp Faradaic peaks, particular to redox reactions relevant to the quinone group in the lignin - see Figure 11, (C) the linear dependence of the peak current, from (B) on the scan rate (horizontal axis) - see Section 3.1, indicates a Faradaic/pseudocapacitive process.

Figure 58 (from Ref. [340]) Reduction-Oxidation reactions typical to quinone groups (shown as a red sphere) in a lignosulfonate (LS)- Polypyrrole (PPy) matrix composite electrode. The water in an aqueous electrolyte systems serves as the proton donor/acceptor to the quinone moiety.

Figure 59 (from Ref. [350]) Three dimensional architectures for electrochemical devices may serve to increase the effective area of charge storage in a compact volume. 
Proposals for such architectures include, e.g., inter-digitated (a) cylindrical or (b) plate like anodes and cathodes, (c) a rod-like array of anodes covered by electrolyte and subsequently the cathode material, (d) aperiodic arrangement where a continuous electrode network is covered with an electrolyte and subsequently an electrode of opposite polarity - affording greater flexibility in wide scale manufacture.

Figure 60 (from Ref. [354]) A monolithic solid-state cell architecture, where a network former provides a continuous matrix for the transfer of $\mathrm{Li}^{+}$to/fro the electrodes via the electrolyte. Two distinct transition metals (e.g., $\mathrm{Ce}^{3+} / \mathrm{Ce}^{4+}$ and $\mathrm{V}^{3+} / \mathrm{V}^{4+}$, which form a part of the network, with widely separated redox potentials) may serve for the electrodes.

Table I A comparison of the characteristics, performance, and cost of the state of the art electrochemical capacitors with a popular battery system

\begin{tabular}{|l|c|c|}
\hline \multicolumn{1}{|c|}{ Characteristic } & $\begin{array}{l}\text { Lithium ion } \\
\text { battery }\end{array}$ & $\begin{array}{l}\text { Electrochemical } \\
\text { capacitor }\end{array}$ \\
\hline Charge/discharge time & $\sim$ minutes & $\sim$ seconds \\
\hline Cycle life & $<5000 @ 1$ C rate & $>500,000$ \\
\hline Energy density $(\mathrm{Wh} / \mathrm{kg})$ & $50-100$ & 5 \\
\hline Power density $(\mathrm{kW} / \mathrm{kg})$ & $0.5-1$ & $5-10$ \\
\hline Cycle efficiency & $<50$ to $>90 \%$ & $<75$ to $>95 \%$ \\
\hline Cost/Wh & $\$ 1-2 / \mathrm{Wh}$ & $\$ 10-20 / \mathrm{Wh}$ \\
\hline Cost/kW & $\$ 75-150 / \mathrm{kW}$ & $\$ 25-50 / \mathrm{kW}$ \\
\hline
\end{tabular}


Table II Charge storage characteristics for a range of generic electroactive polymers.[333]

\begin{tabular}{|l|l|l|l|l|}
\hline Types of Polymer & $\begin{array}{l}\text { Charge Density } \\
(\mathrm{Ah} / \mathrm{kg})\end{array}$ & $\begin{array}{l}\text { Specific Energy } \\
(\mathrm{Wh} / \mathrm{kg})\end{array}$ & $\begin{array}{l}\text { Open Circuit Voltage } \\
(\mathrm{V})\end{array}$ & $\begin{array}{l}\text { Specific Power } \\
\text { (W/kg) }\end{array}$ \\
\hline Polyacetylenes & $100-300$ & $100-300$ & $3.5-3.9$ & up to 30,000 \\
\hline Polyaniline & $50-150$ & $100-350$ & $1-4$ & \\
\hline Polypyrrole & $50-120$ & $50-350$ & $3-4$ & \\
\hline Polythiophene & $25-100$ & $50-325$ & $3.2-4.2$ & up to 90,000 \\
\hline Poly-p-phenylene & $20-140$ & 300 & $3.2-4.5$ & up to 320 \\
\hline
\end{tabular}




\section{Charge transfer and storage in nanostructures}

P. R. Bandaru, H. Yamada, R. Narayanan, and M. Hoefer

Program in Materials Science, Department of Mechanical Engineering,

University of California, San Diego, La Jolla, CA 92093-0411 
Figure 1

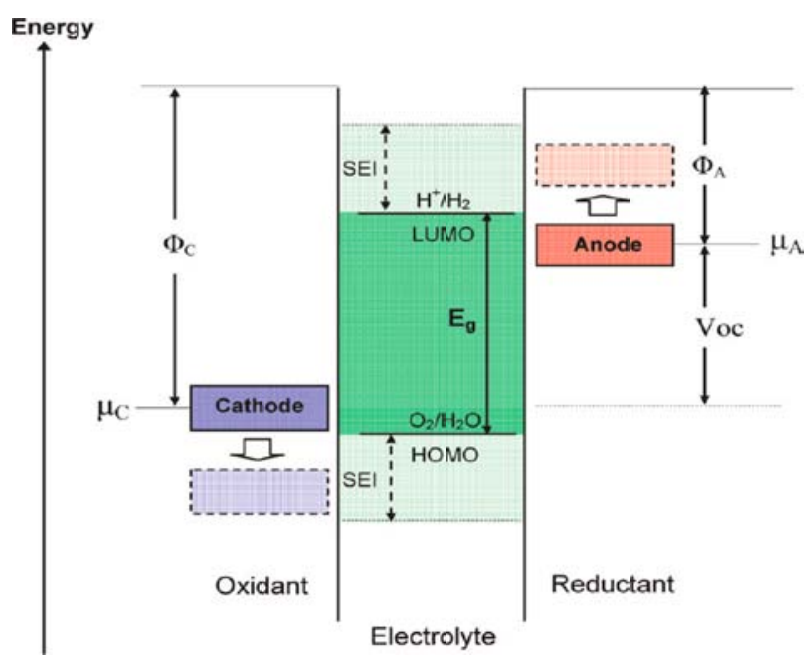


Figure 2

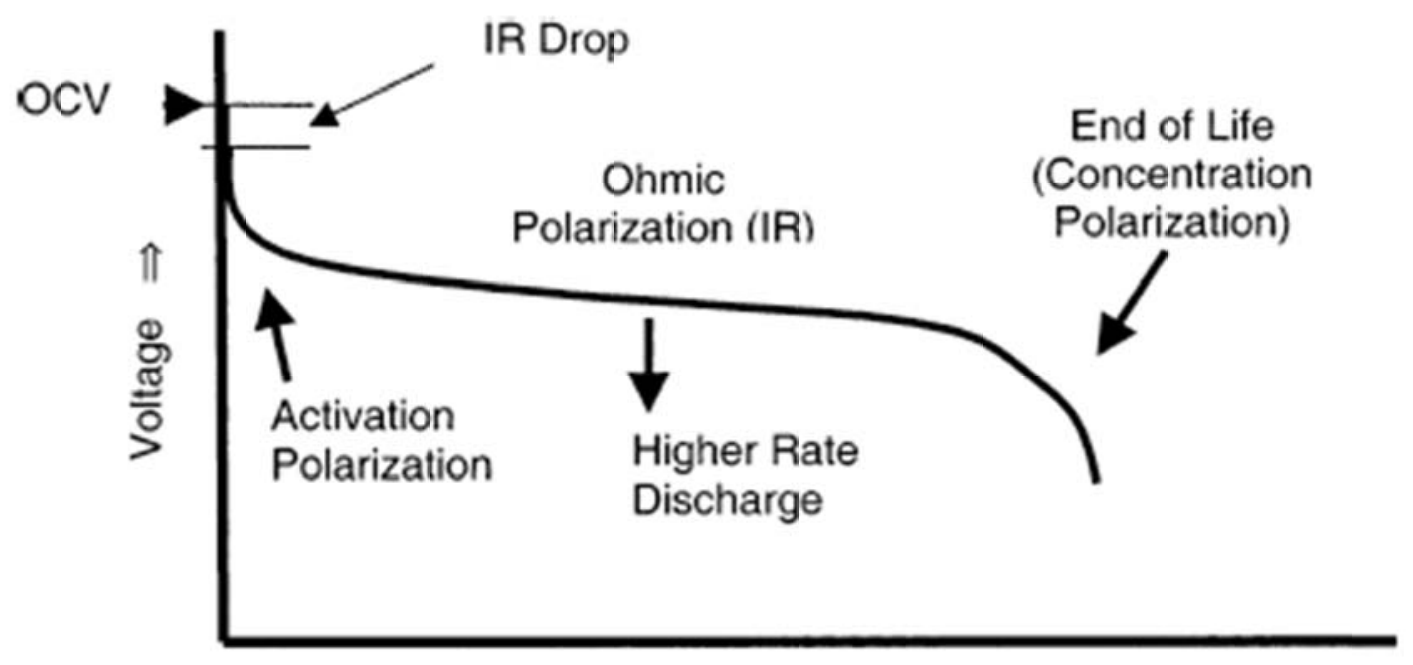

Discharge $\Rightarrow$ 
Figure 3

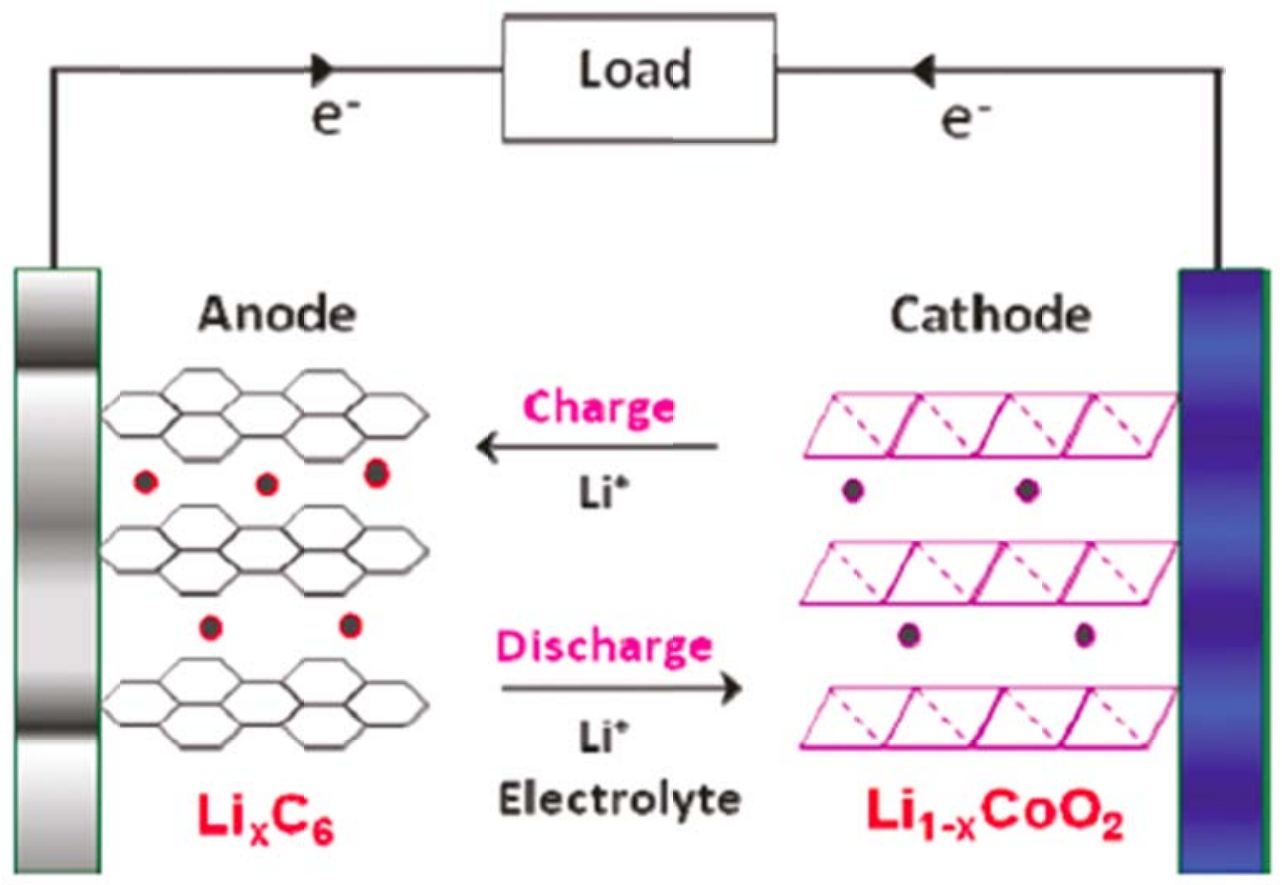




\section{Figure 4}

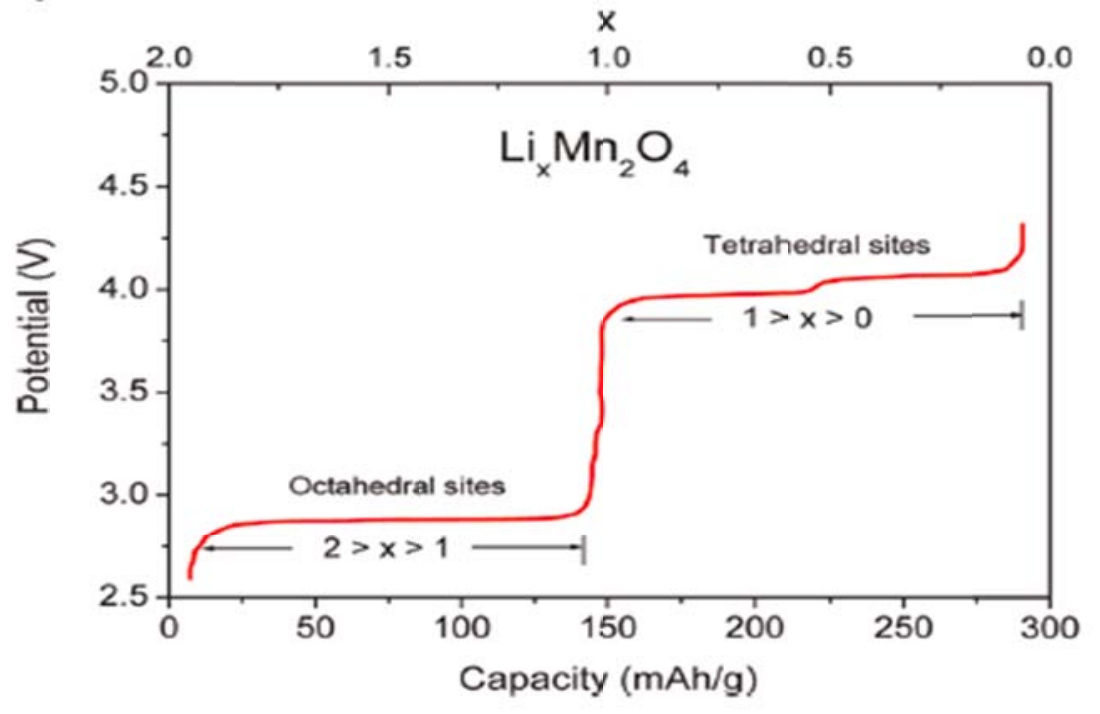


Figure 5

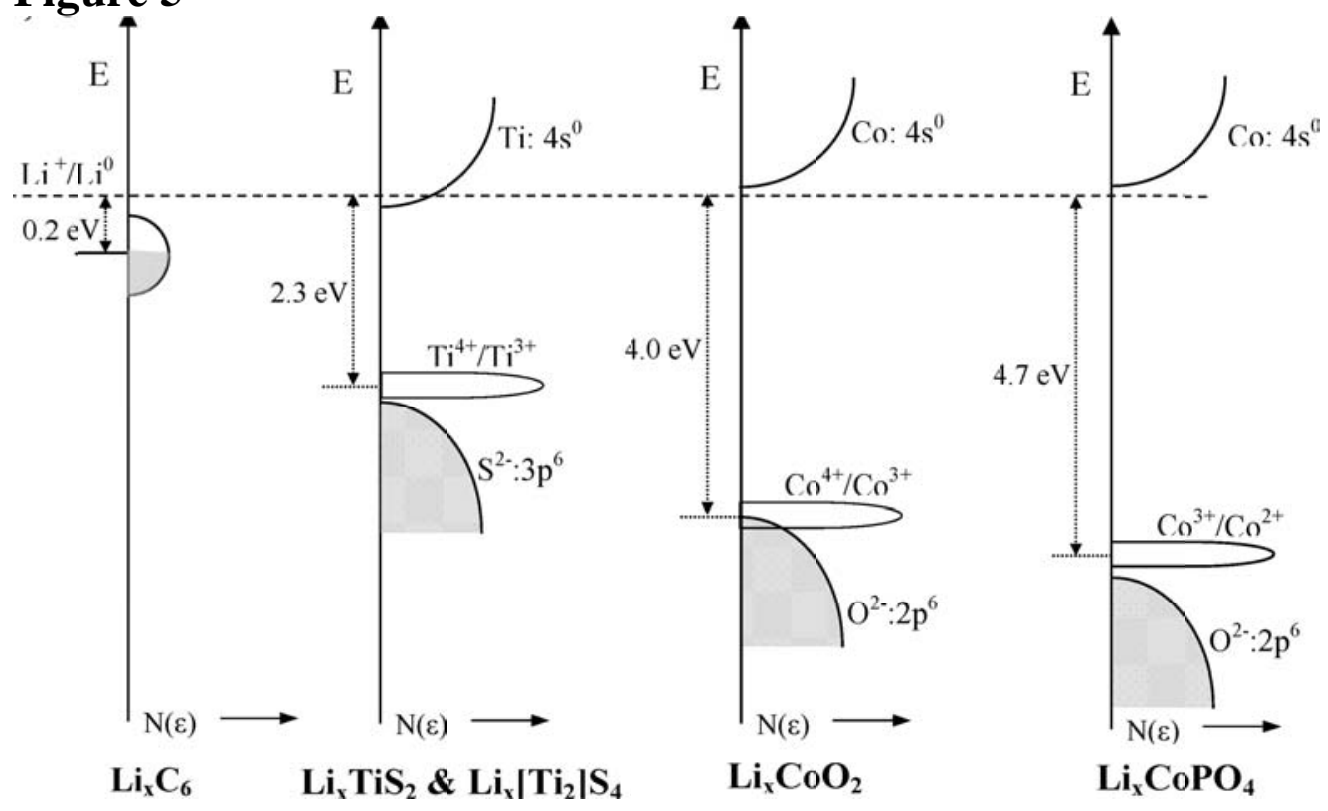


Figure 6

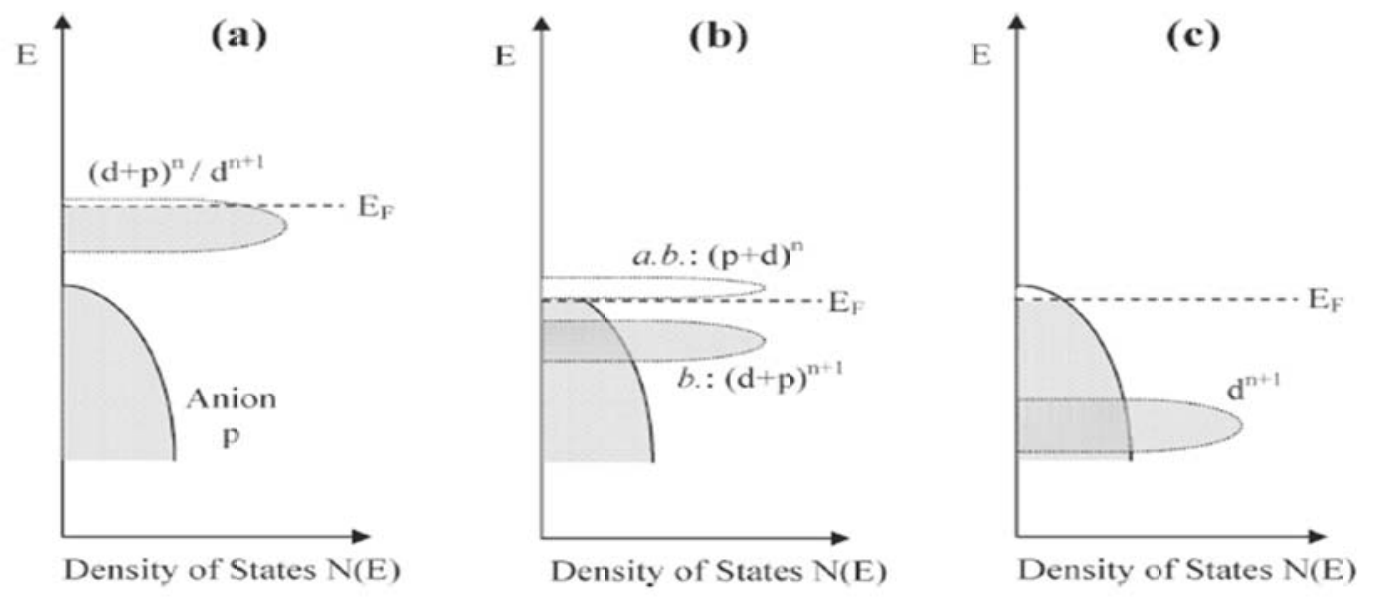


Figure 7

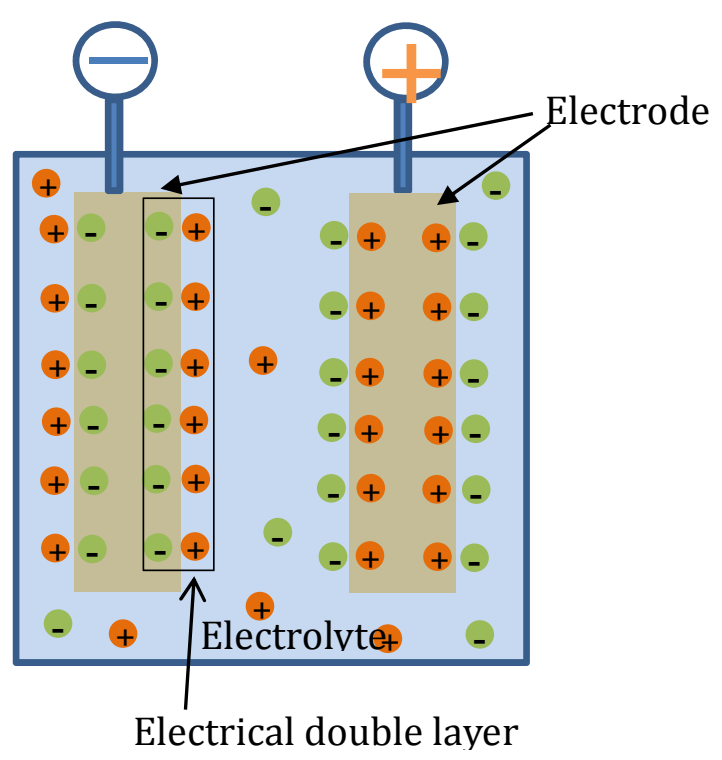




\section{Figure 8}

(a)

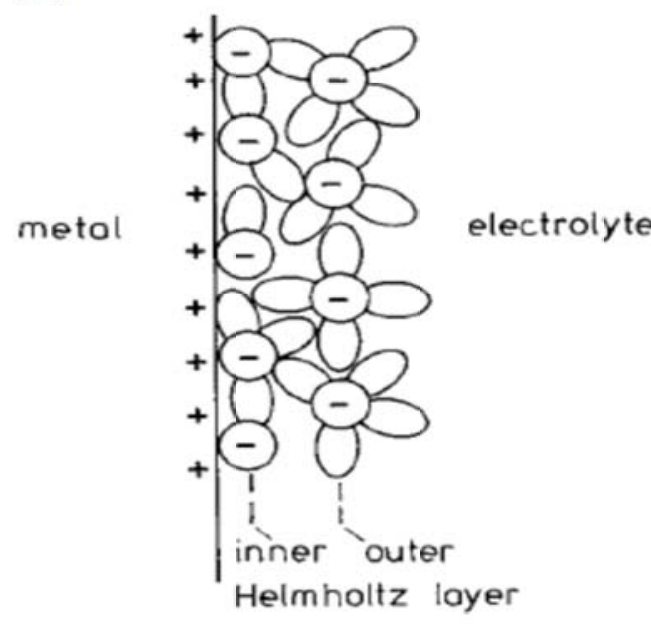

(b)

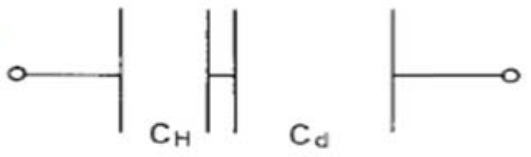

(c)

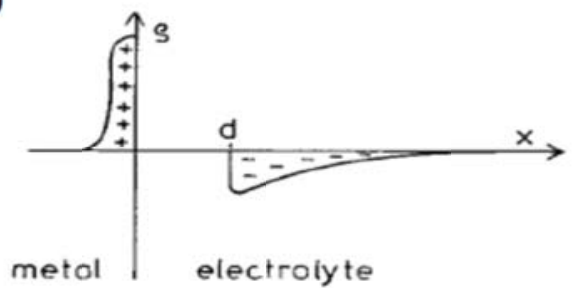

(d)

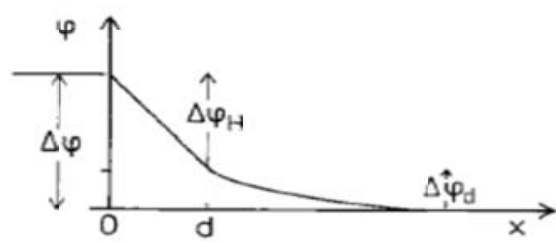


Figure 9
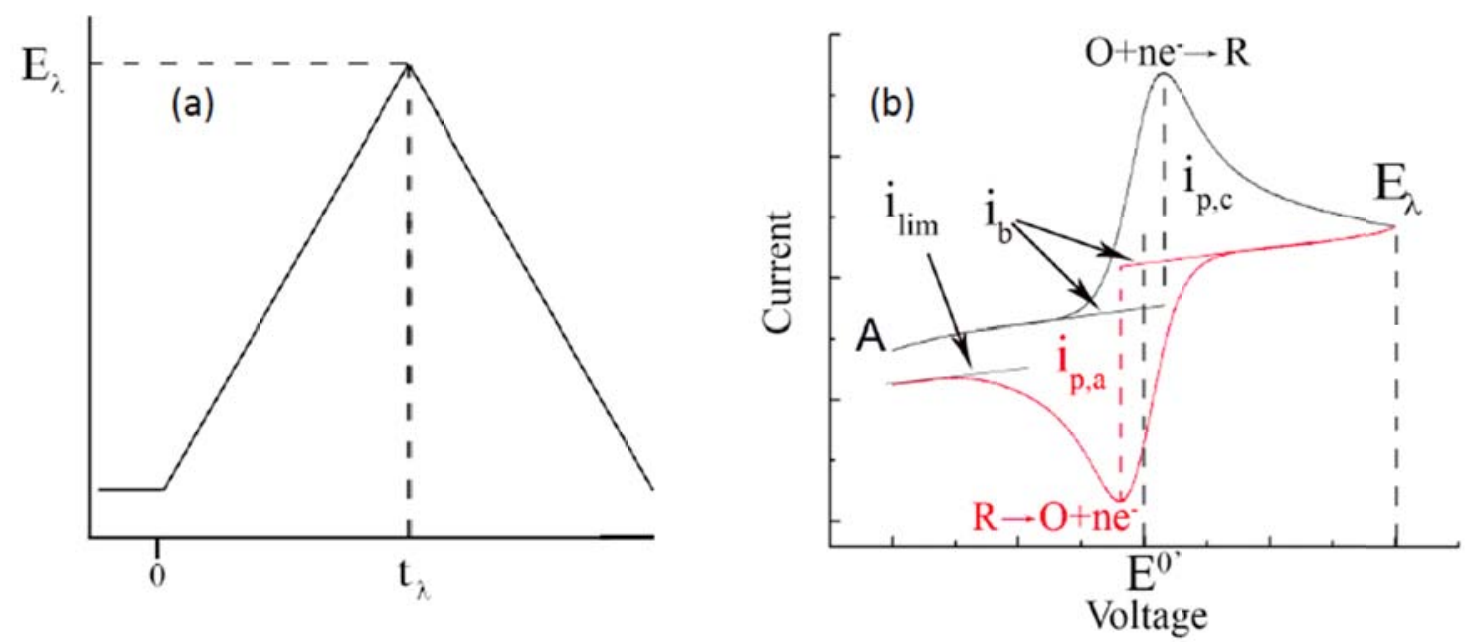
Figure 10

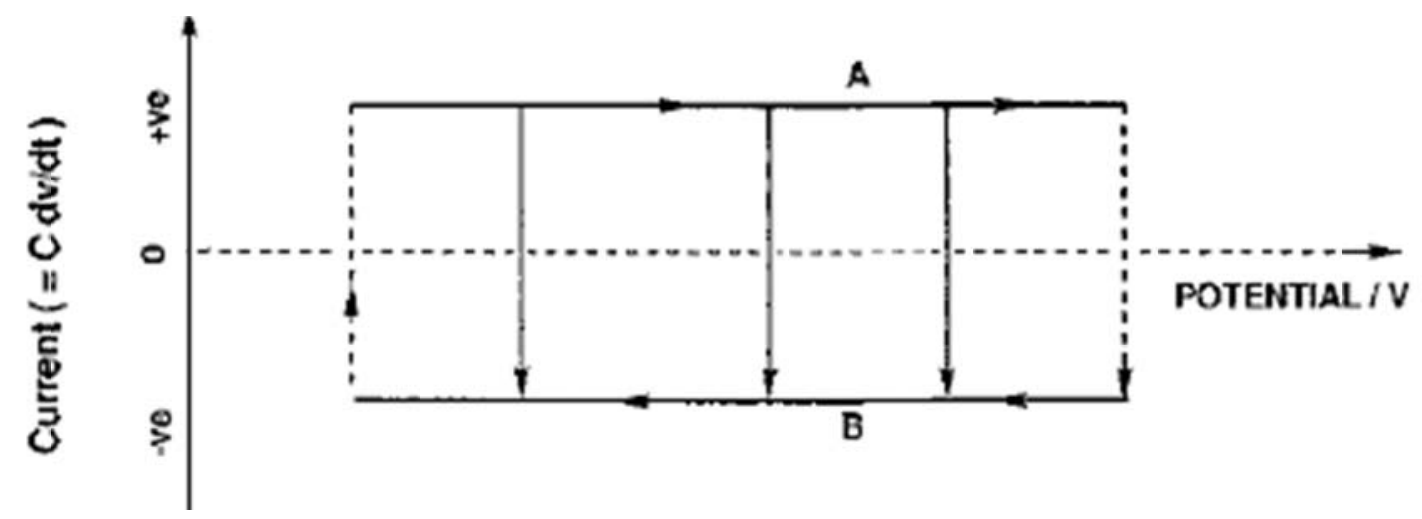


Figure 11

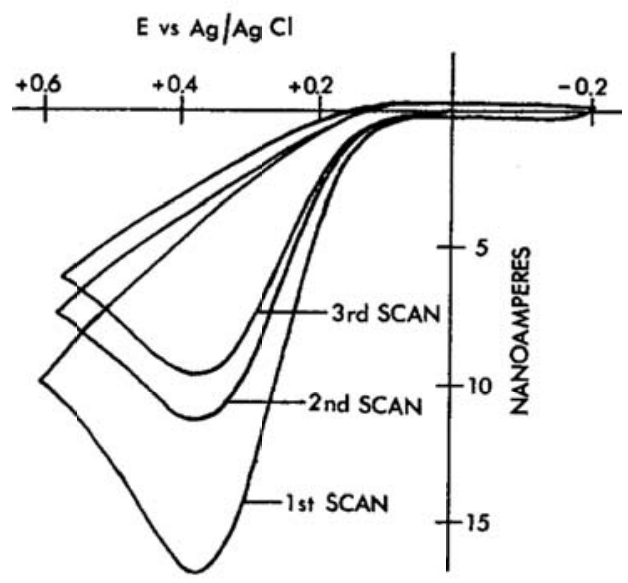


Figure 12
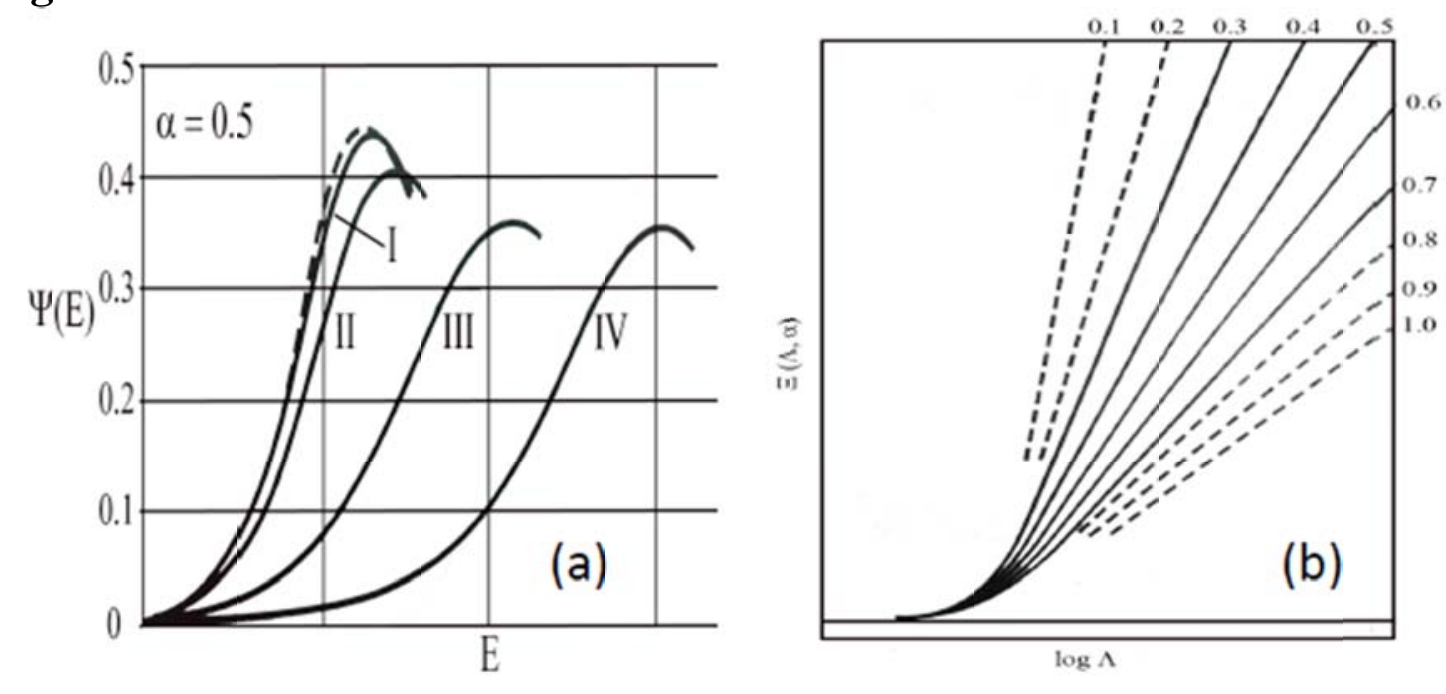
Figure 13

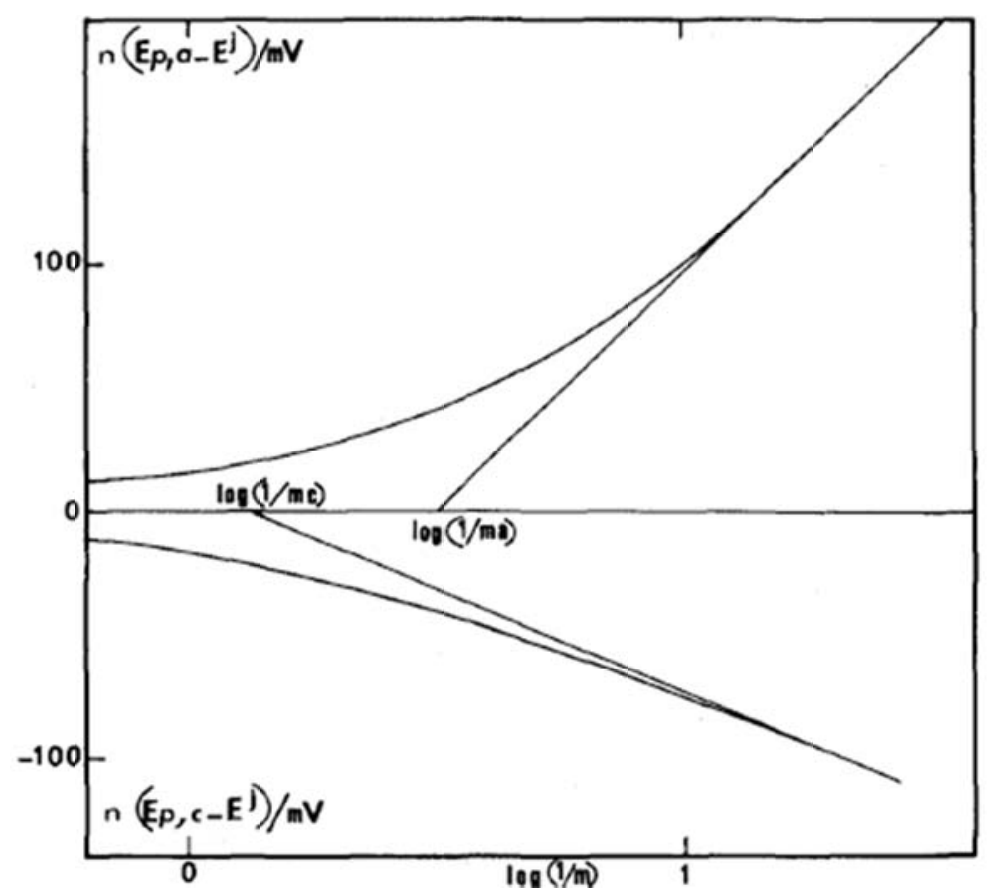


Figure 14
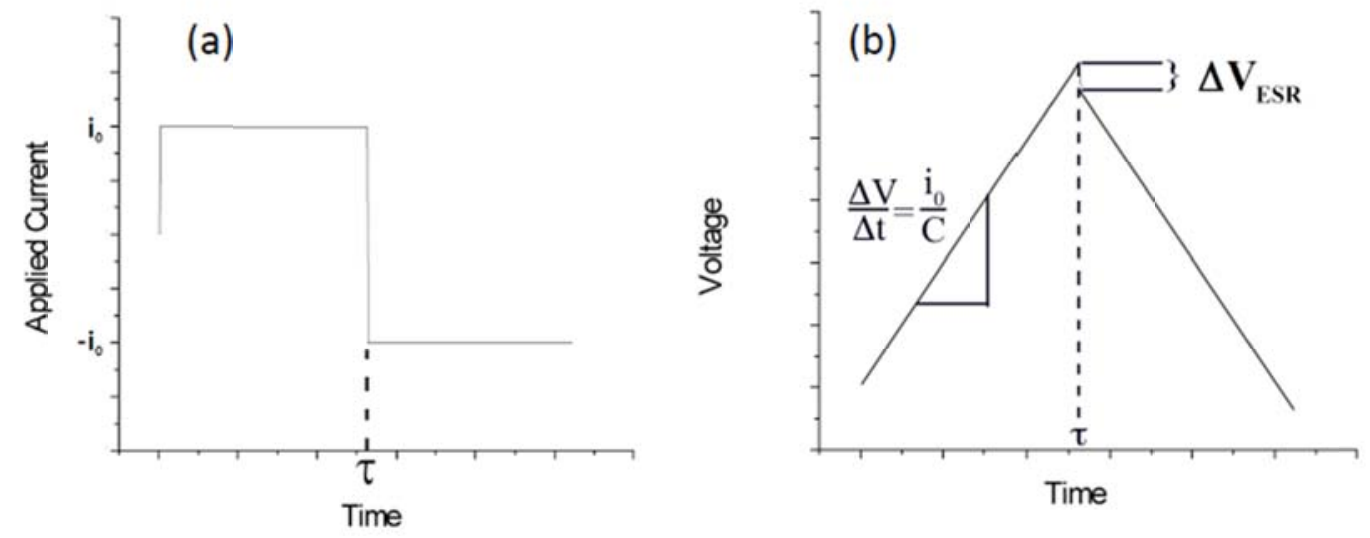
Figure 15

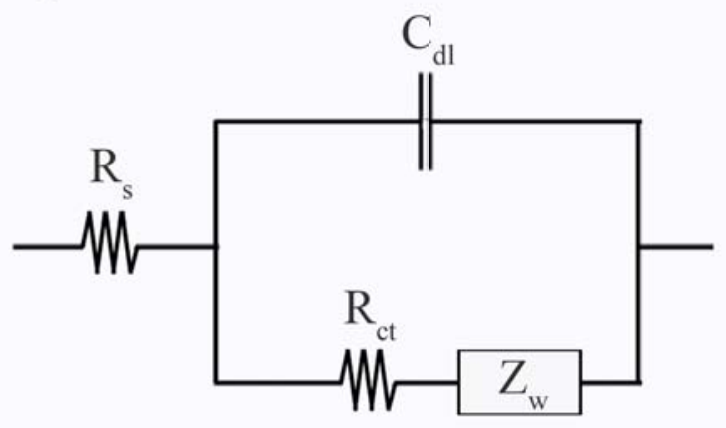


Figure 16

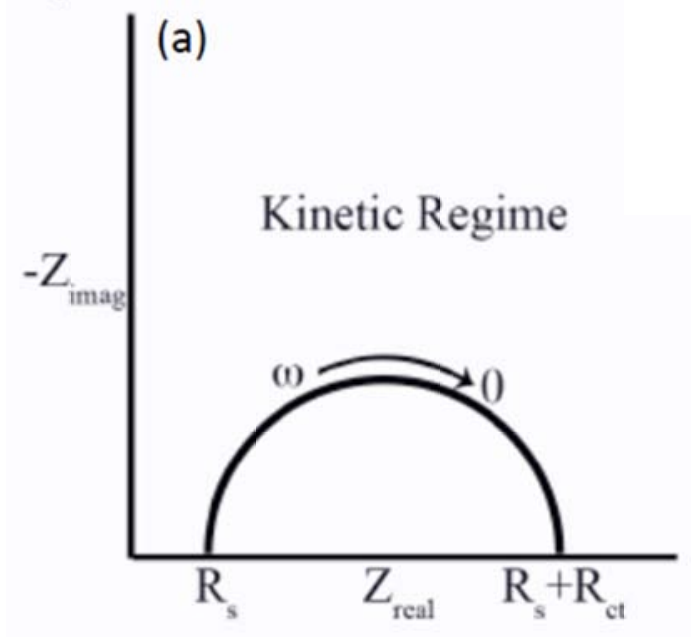

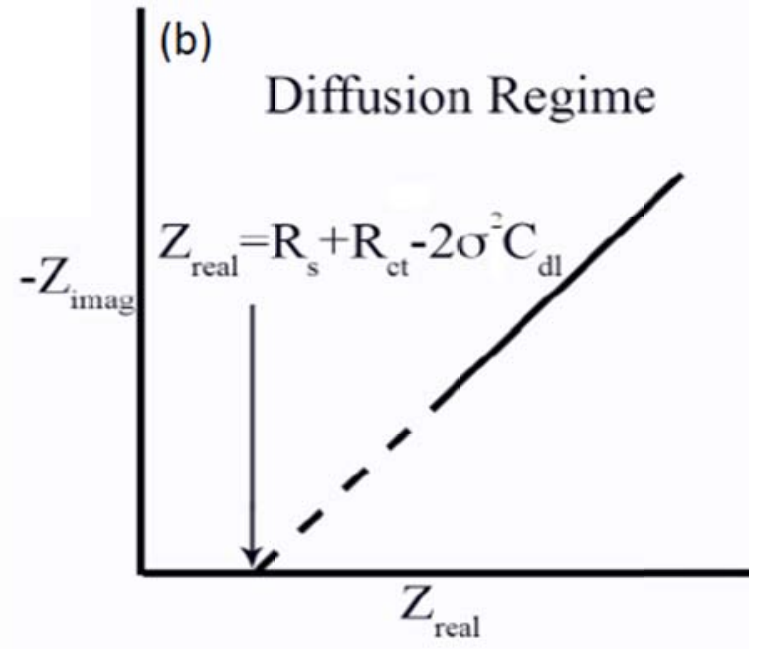


Figure 17
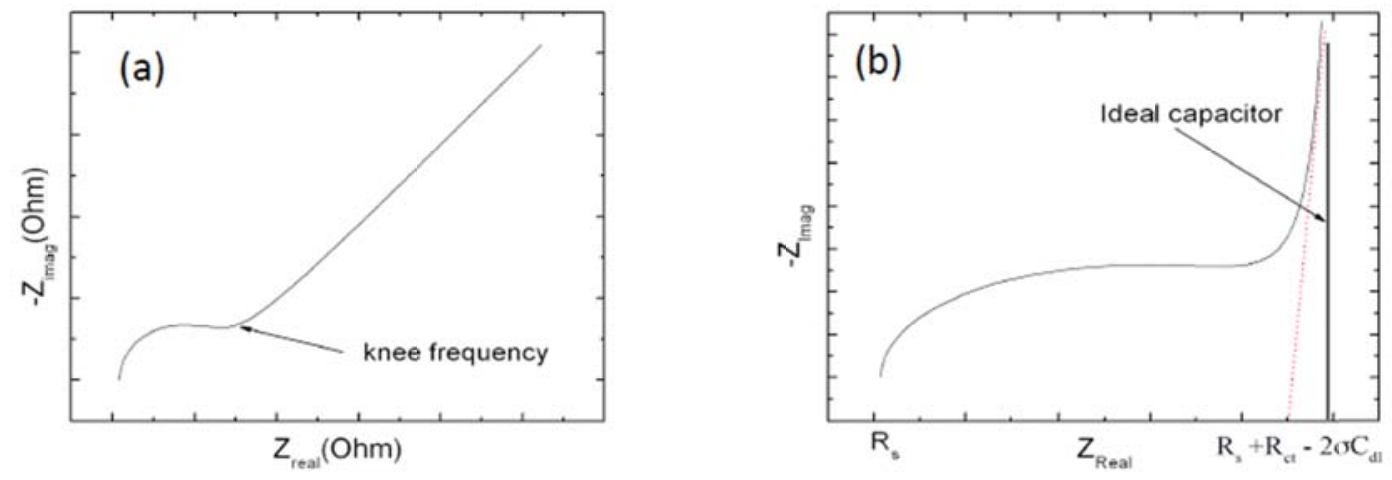
Figure 18

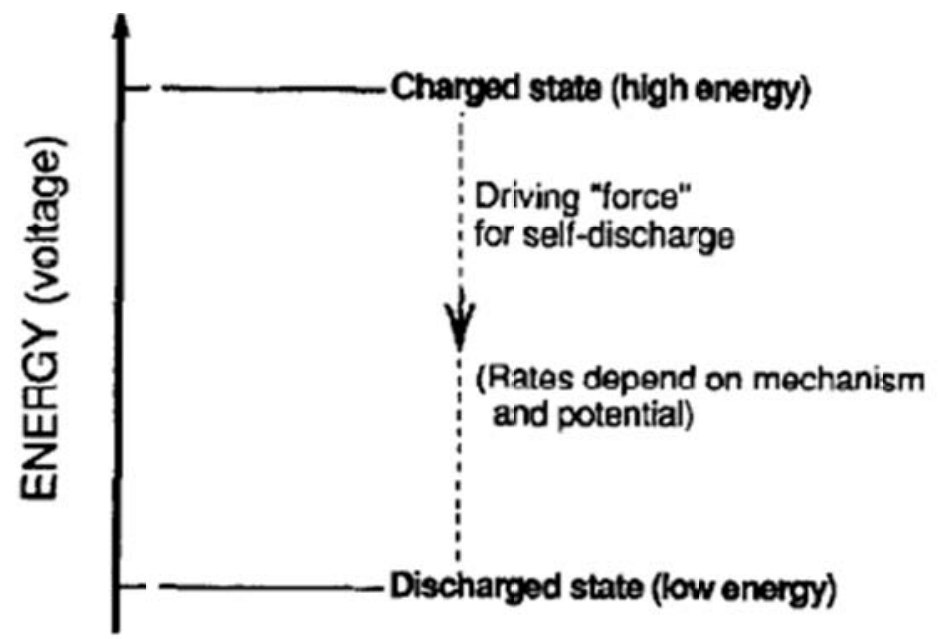


Figure 19

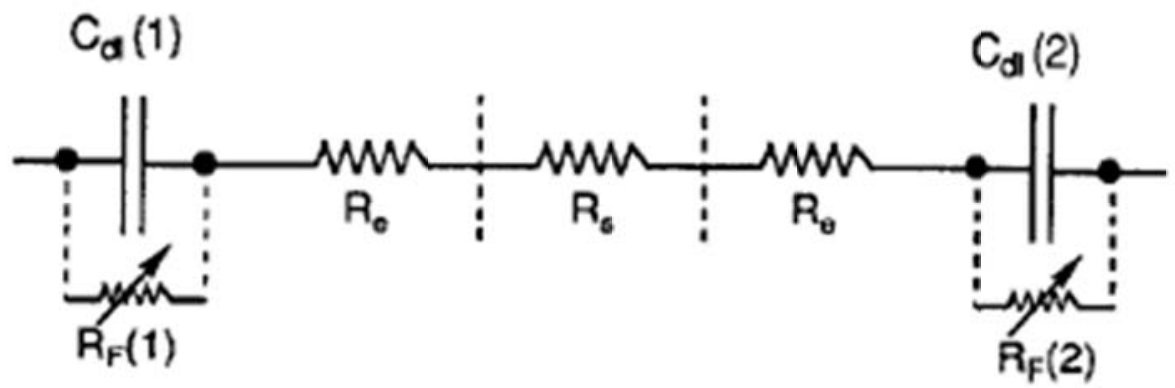


Figure 20

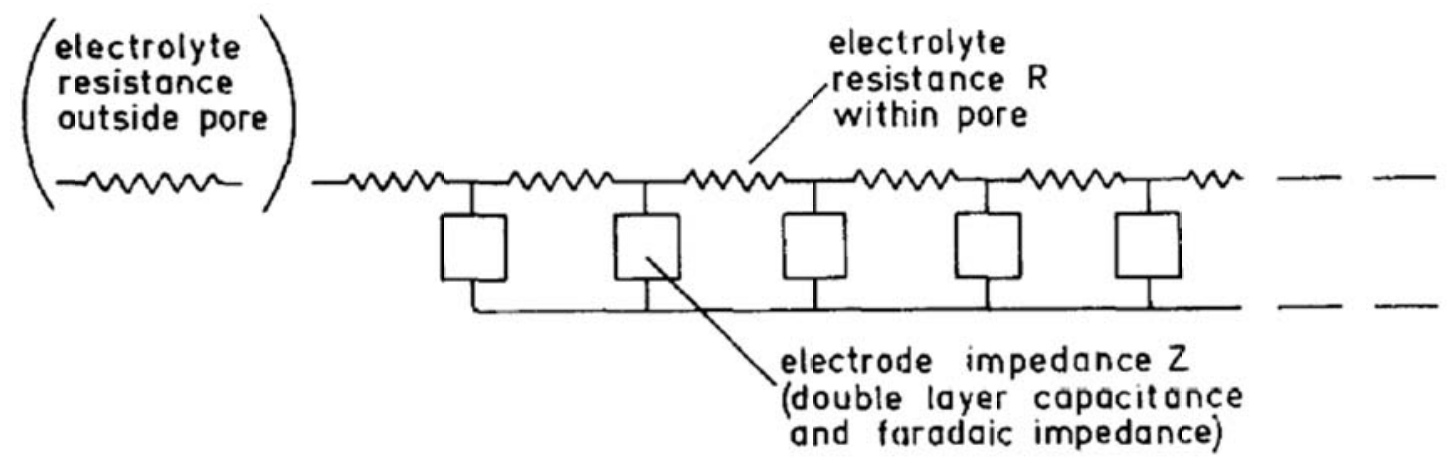


Figure 21

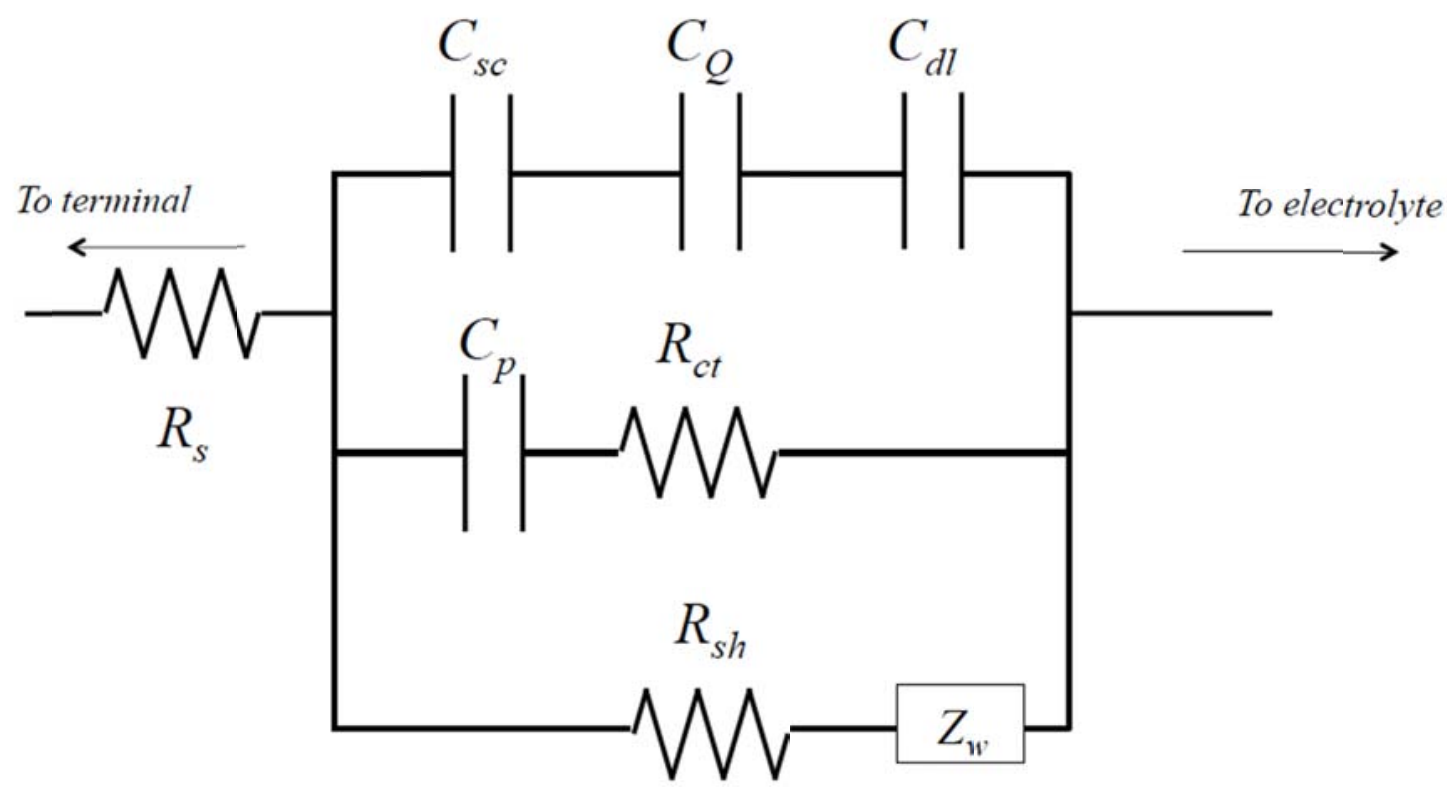


Figure 22

(a)

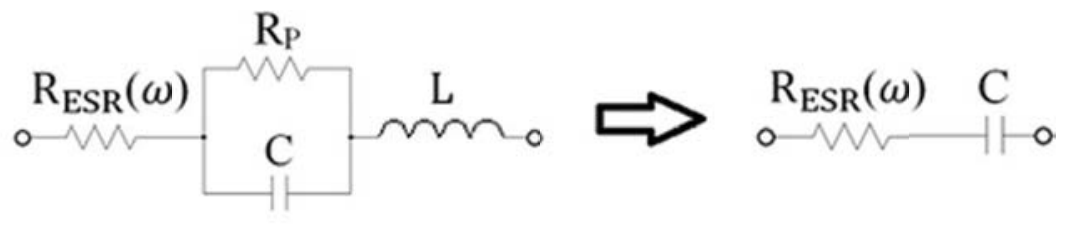

(b)

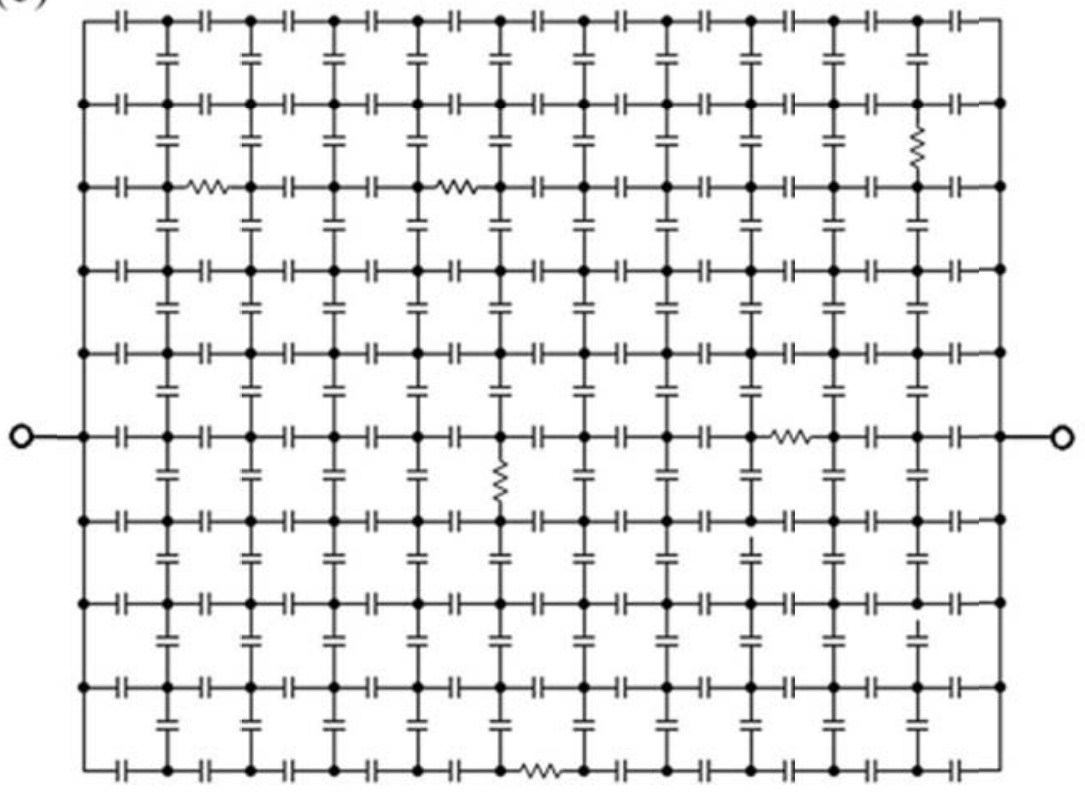


Figure 23

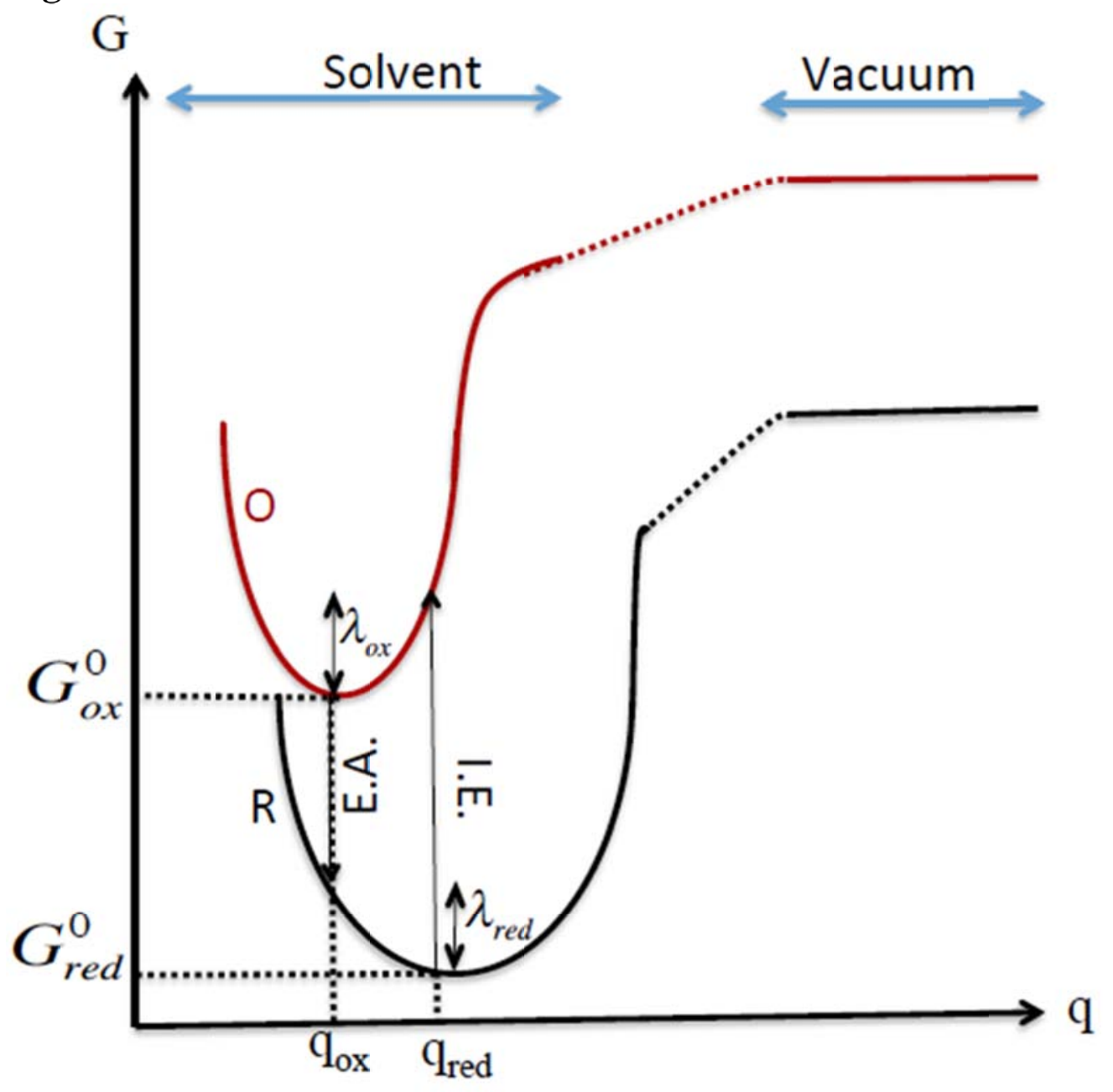


Figure 24

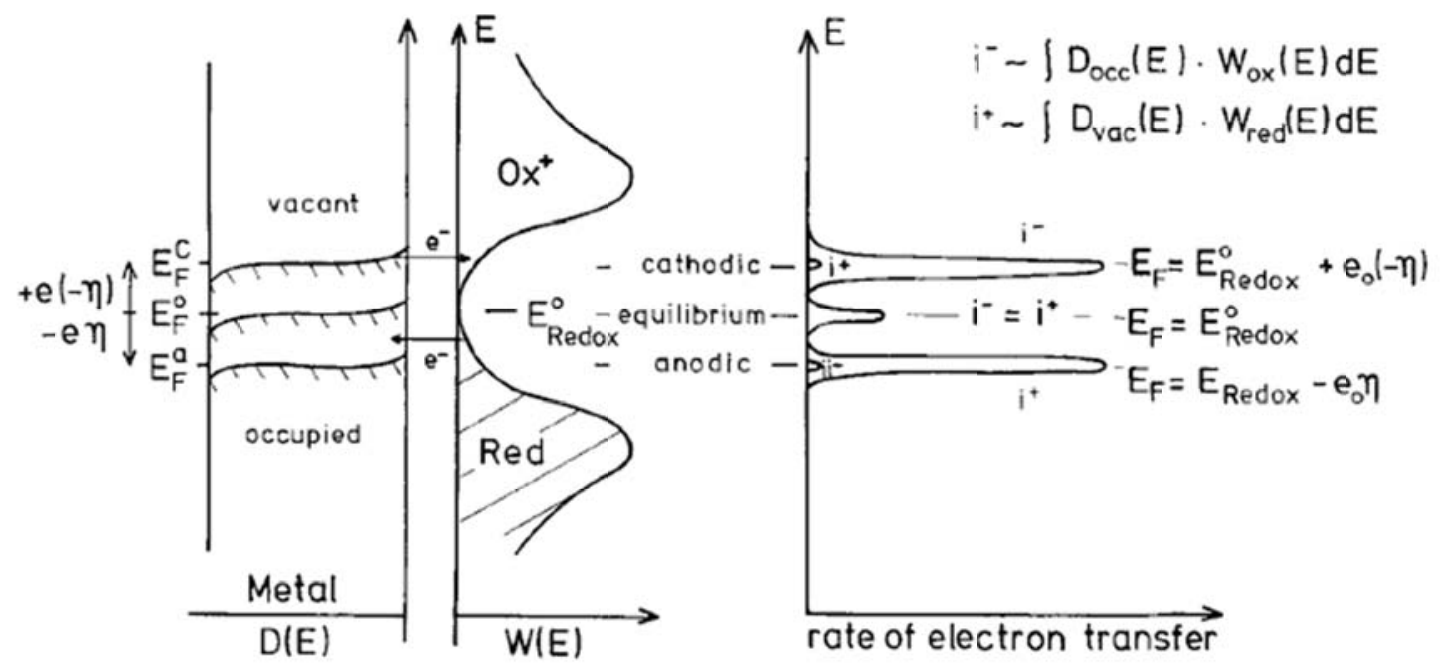


Figure 25

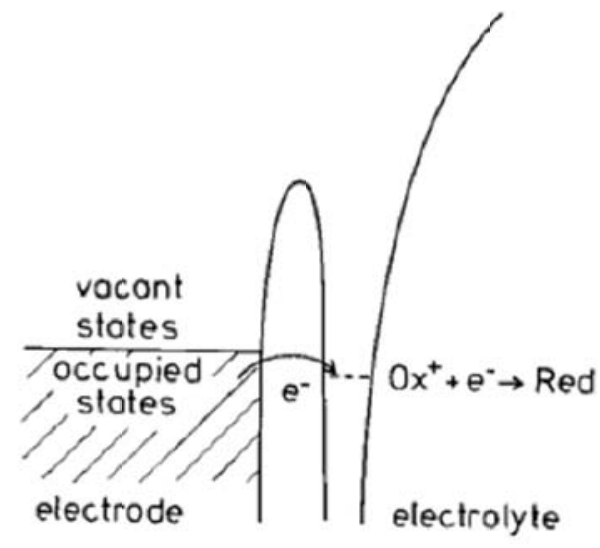

Reduction

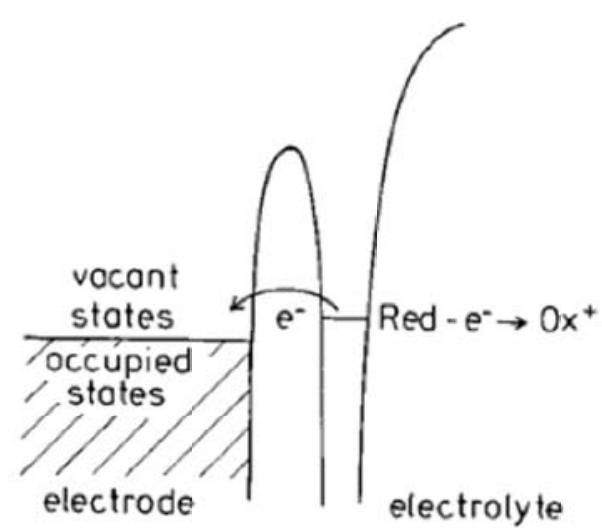

Dxidation 
Figure 26

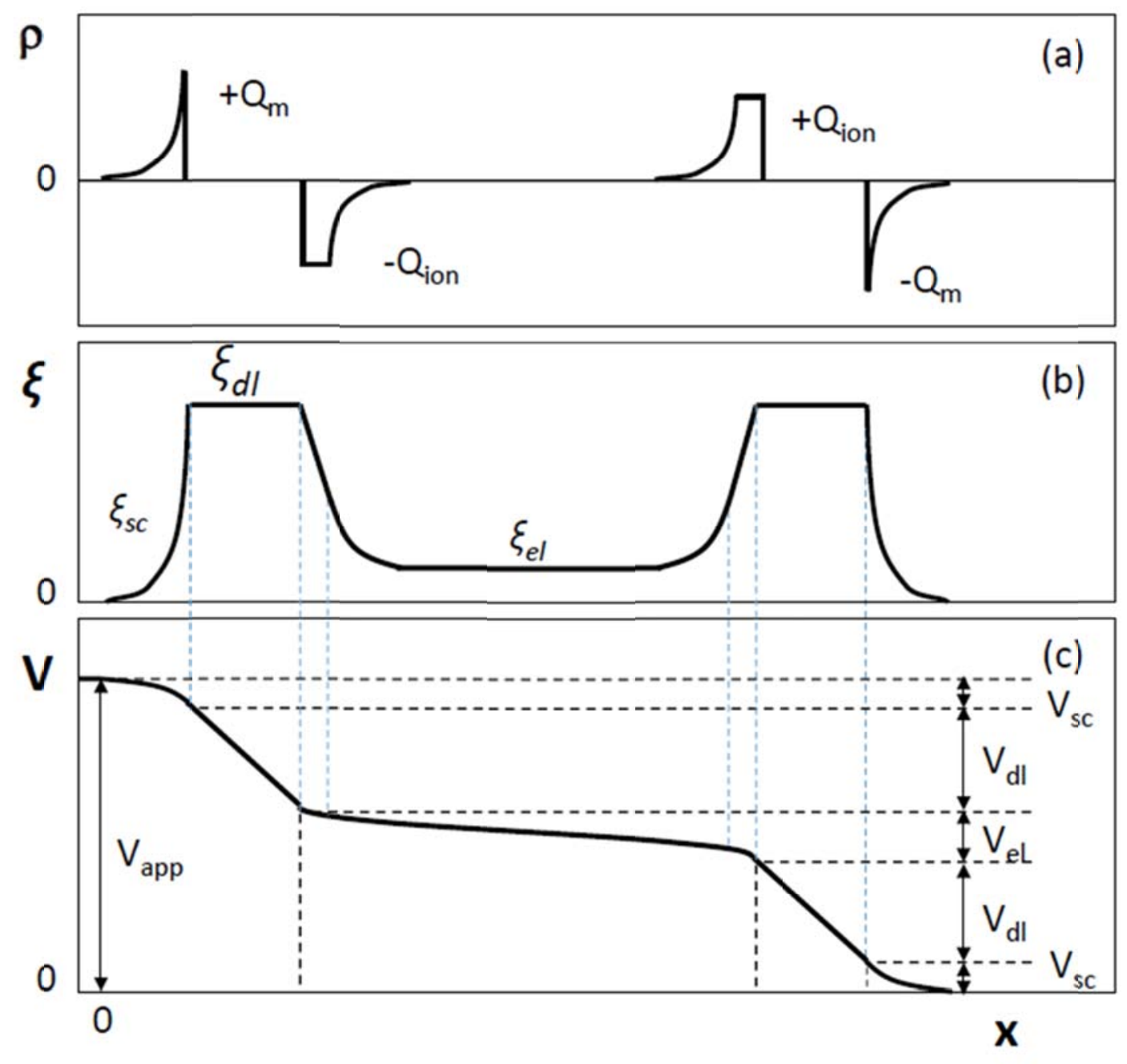


Figure 27

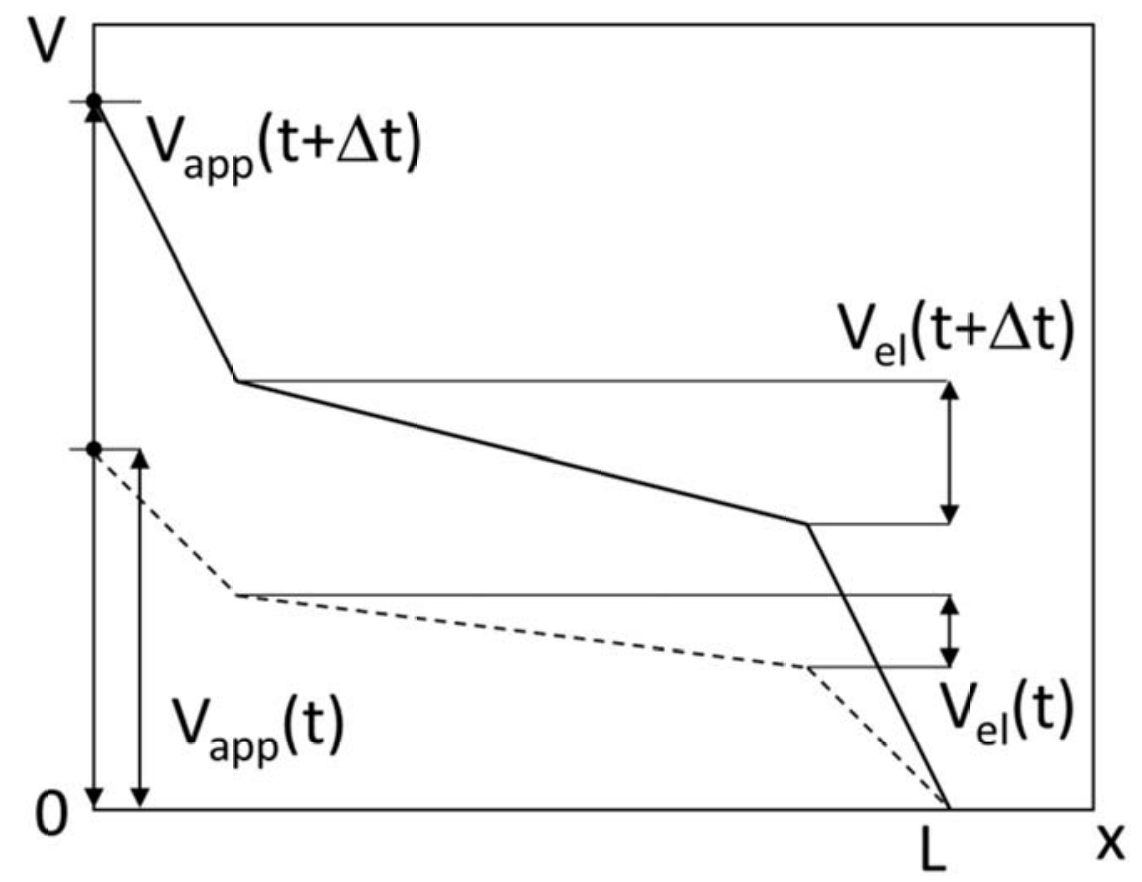


Figure 28

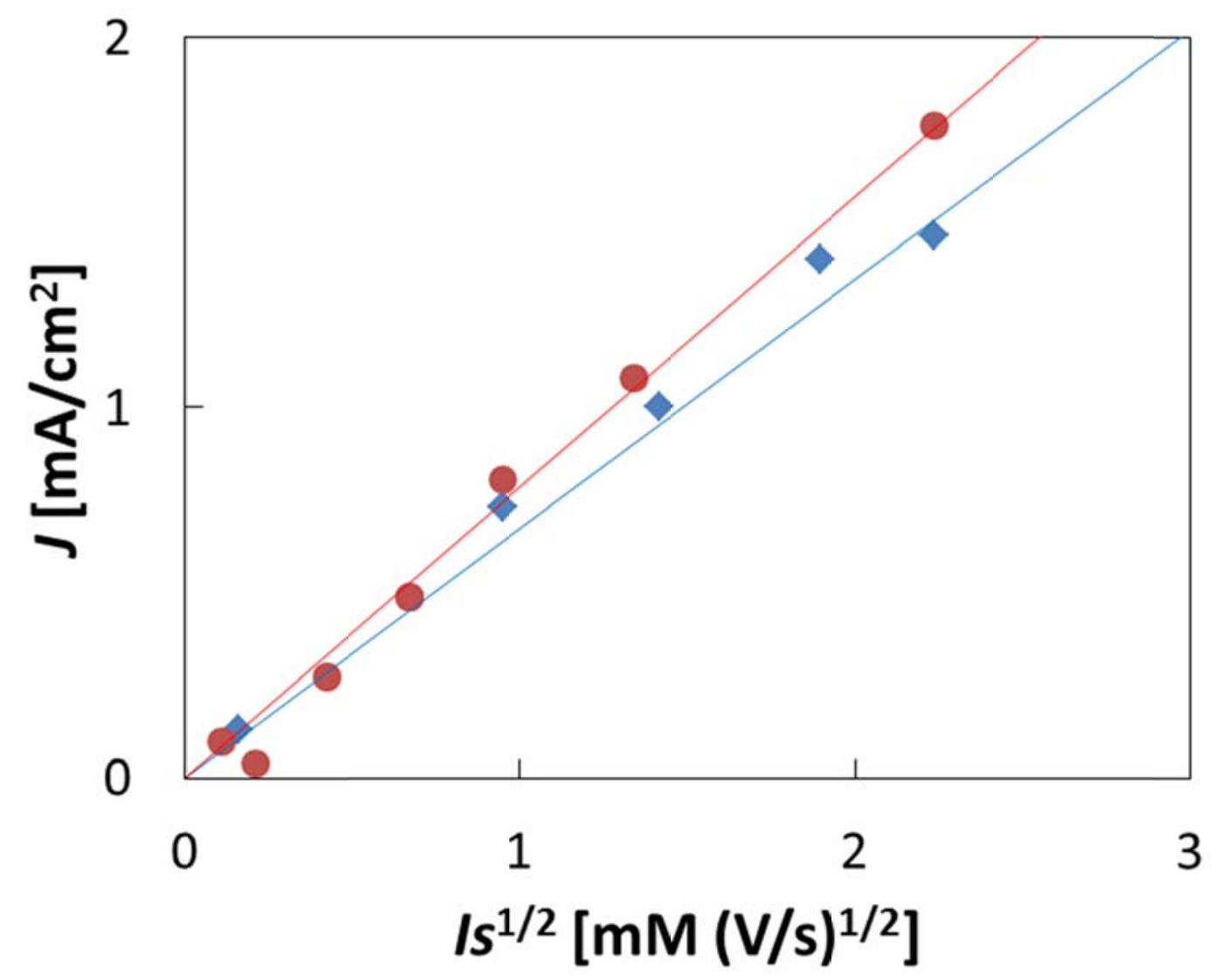


Figure 29

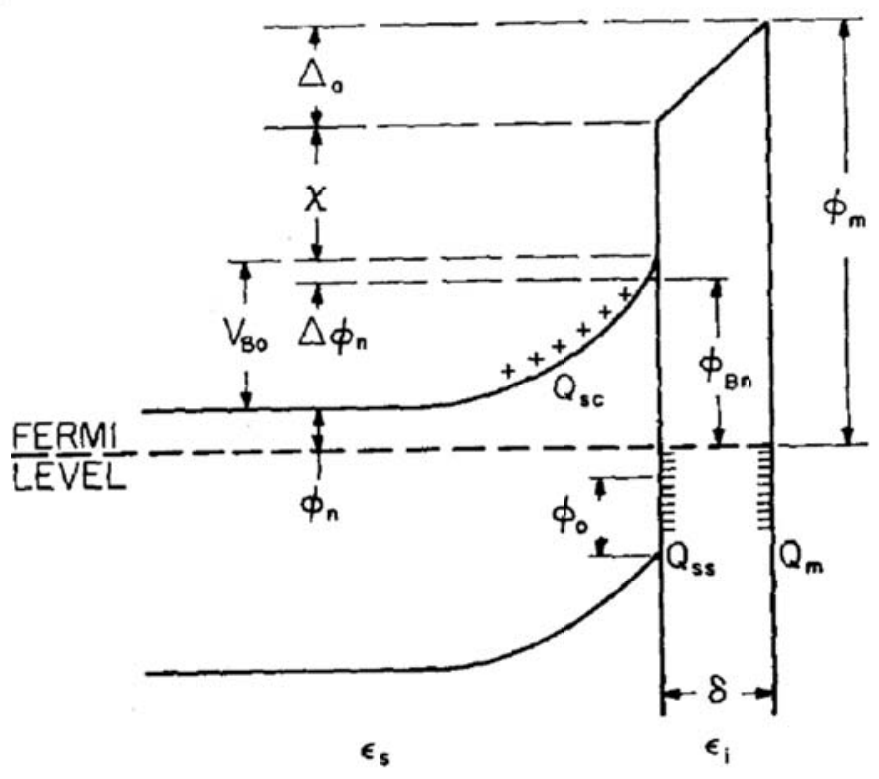


Figure 30

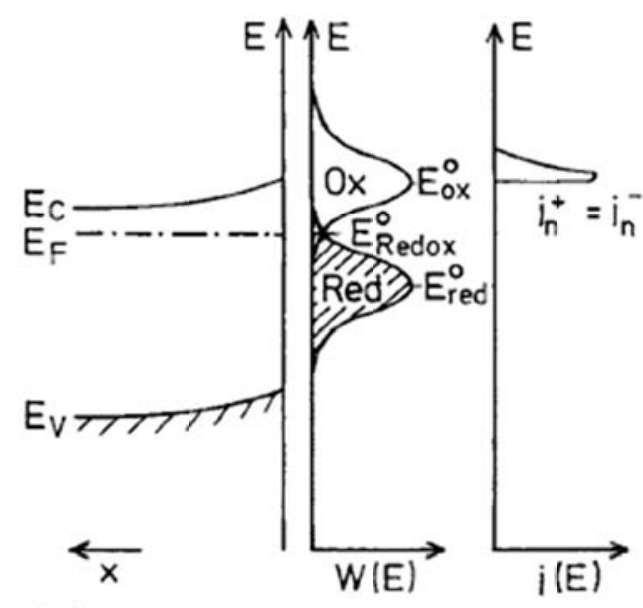

(a)

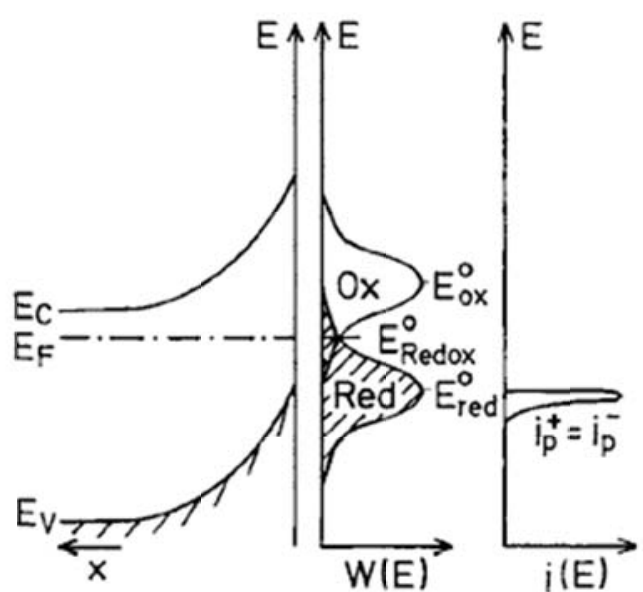

(b) 
Figure 31
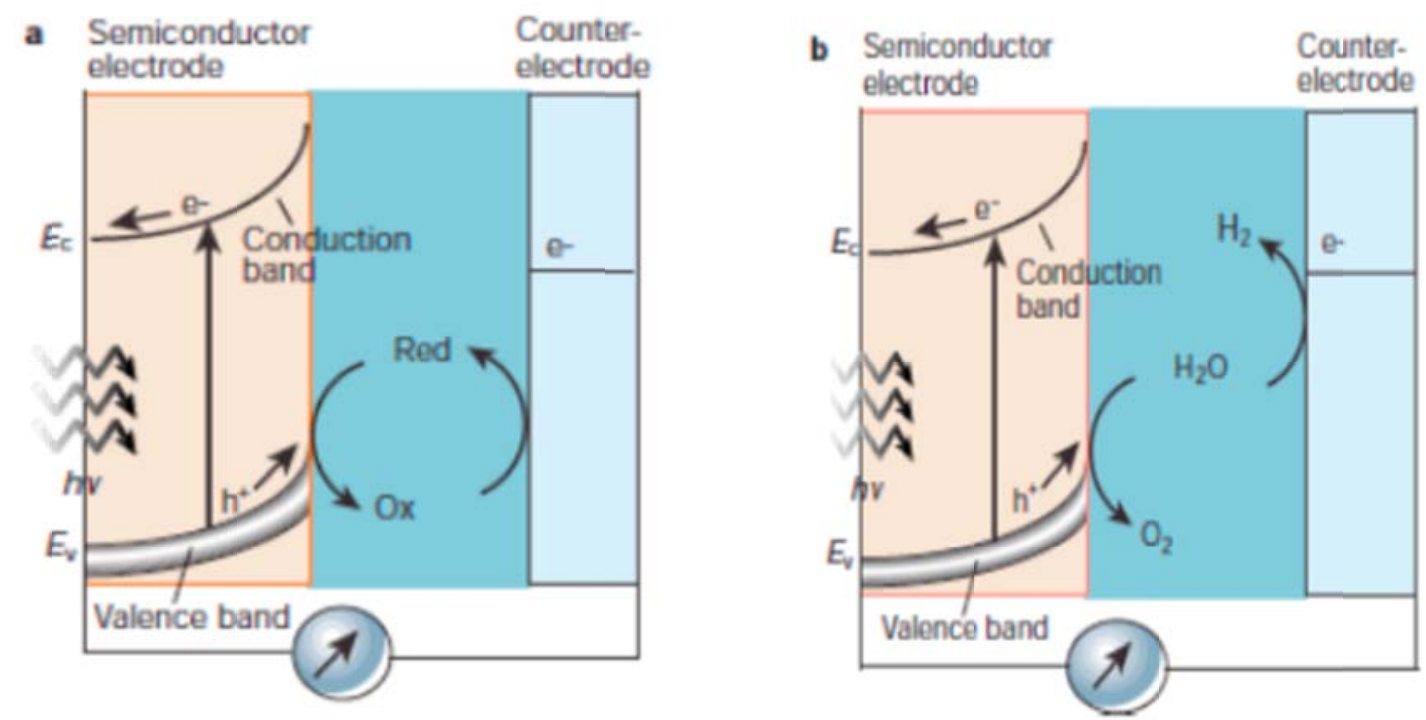
Figure 32
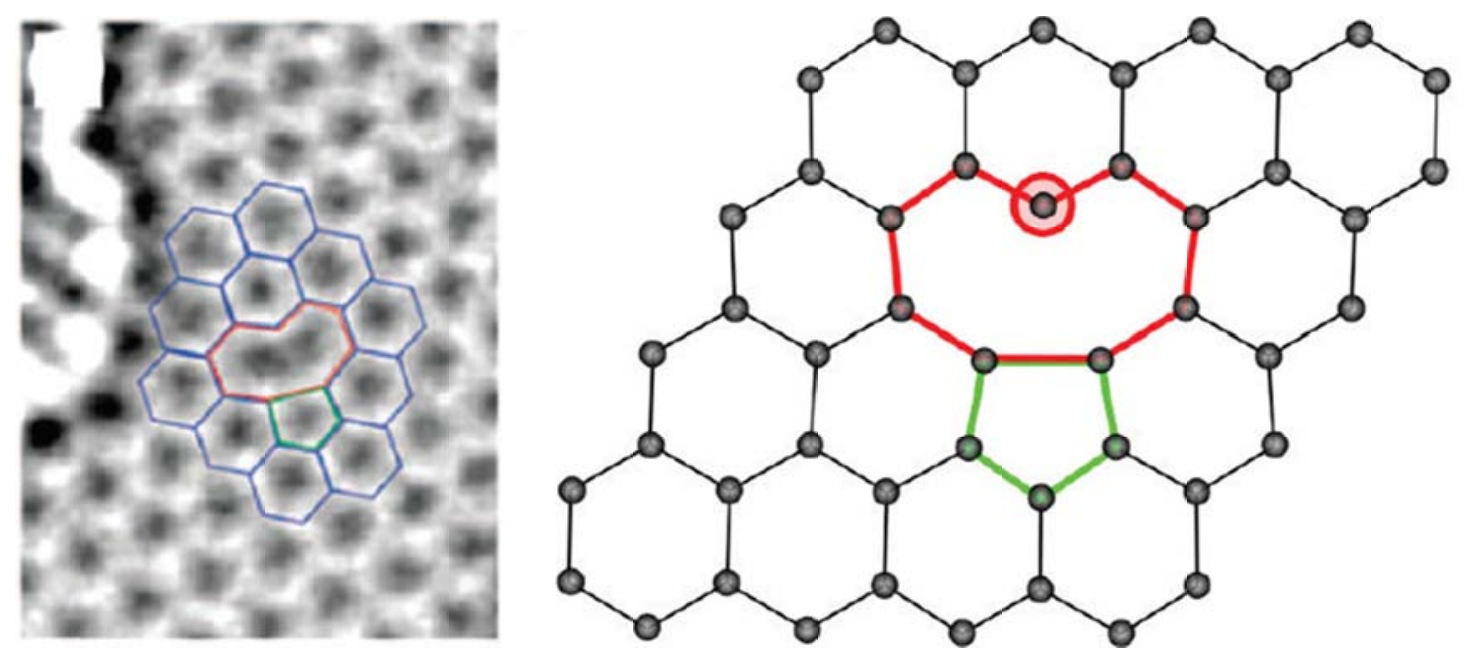
Figure 33

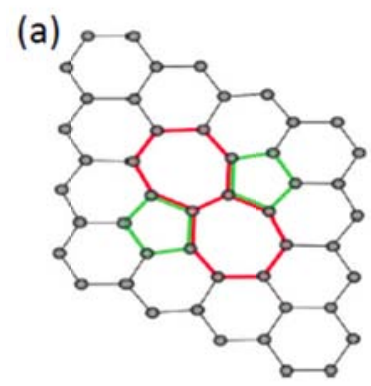

(b)

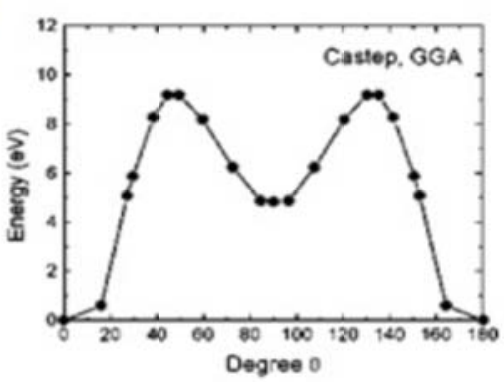

(c)

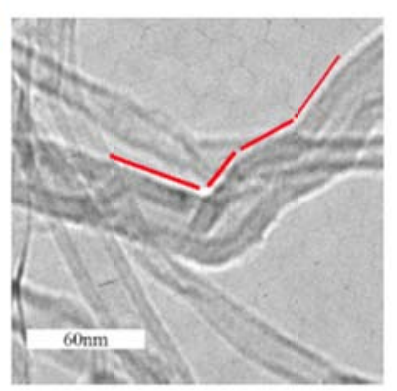


Figure 34

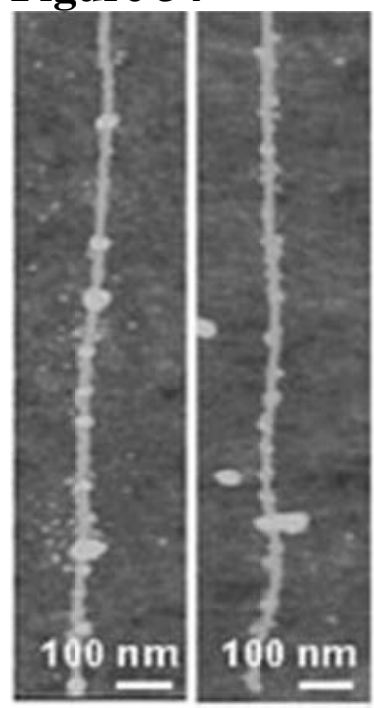

Work function (V)

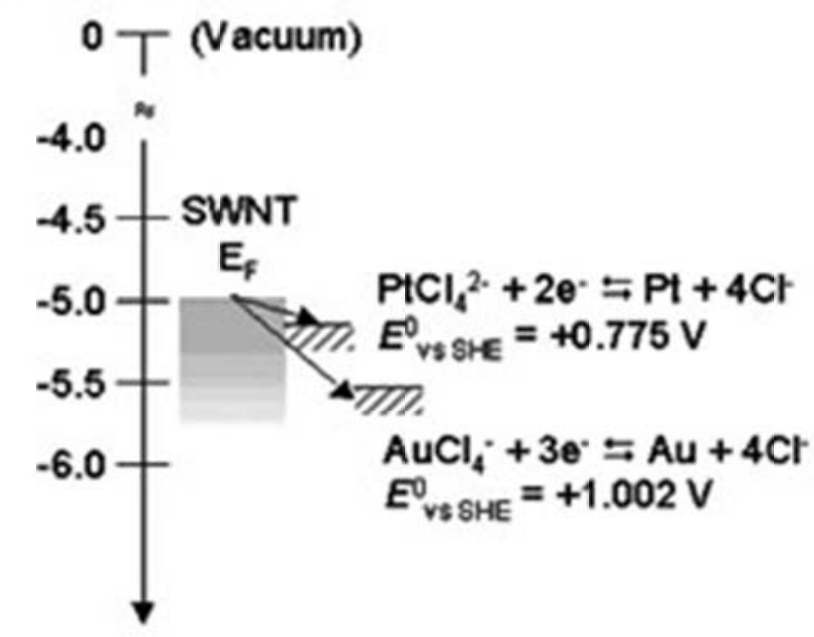


Figure 35
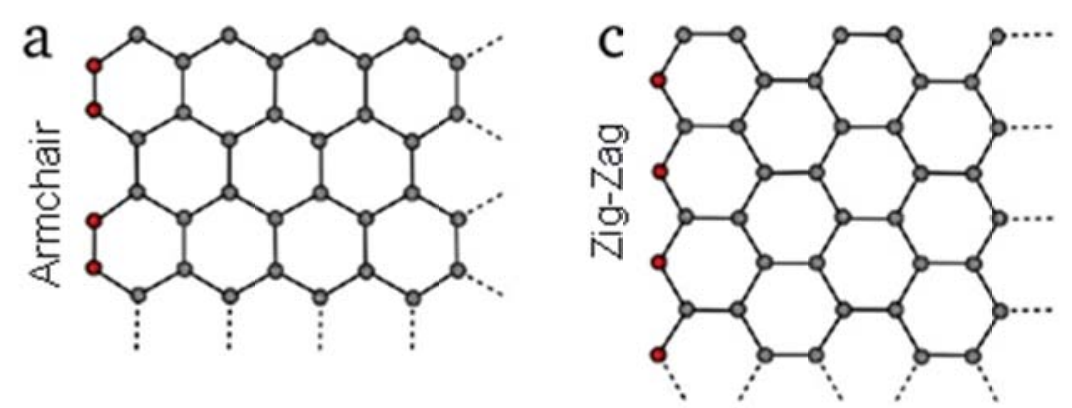

b
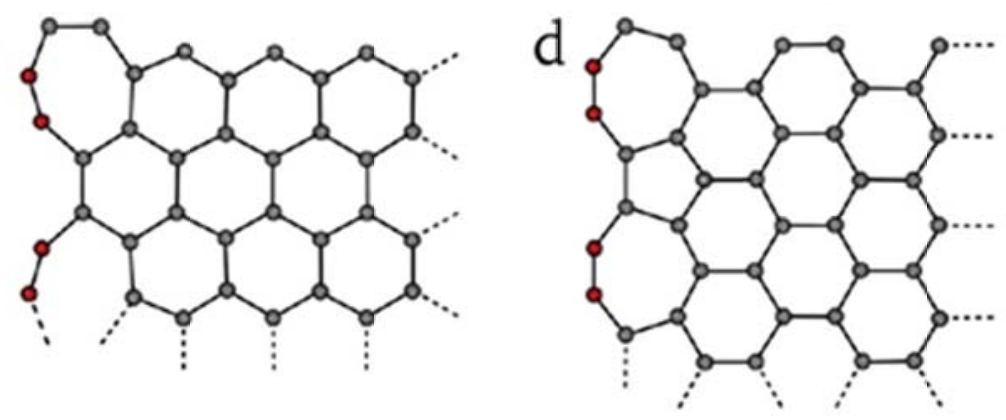
Figure 36

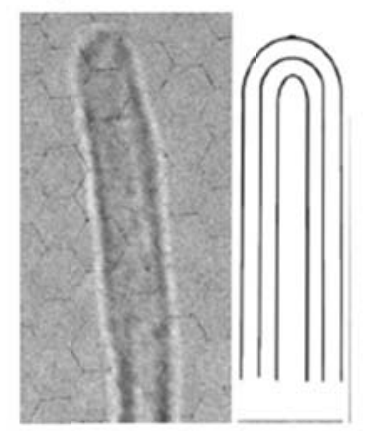

(a)

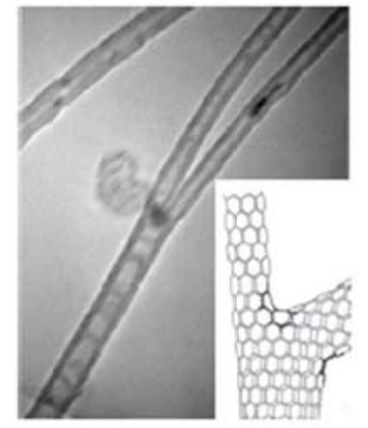

(b)

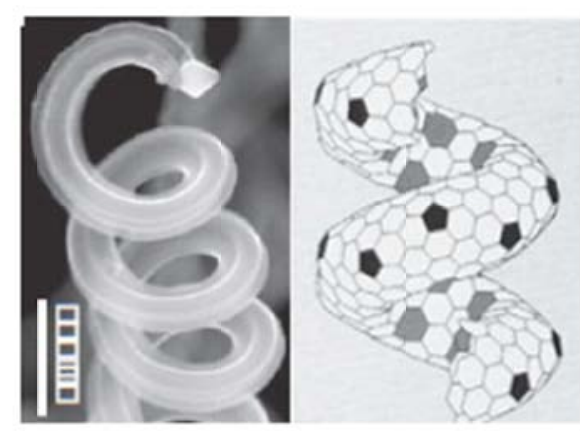

(c)

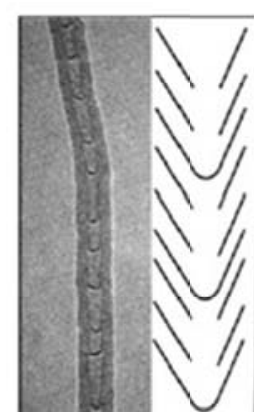

(d)

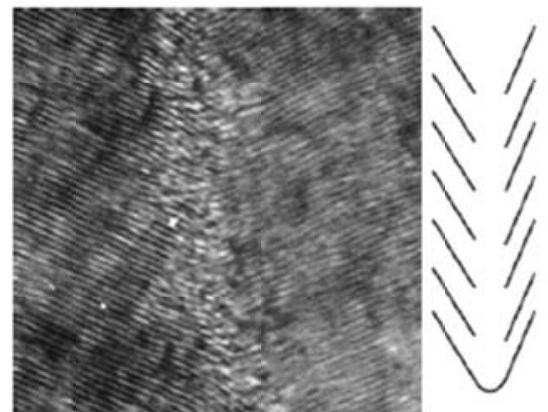

(e) 
Figure 37

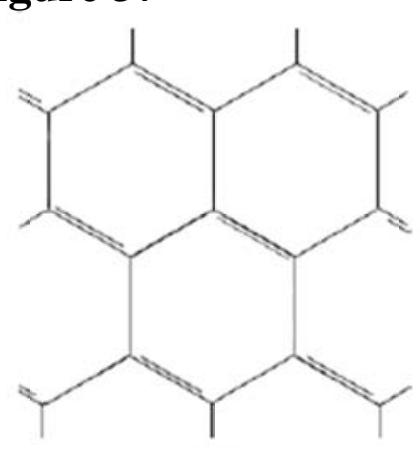

(a)

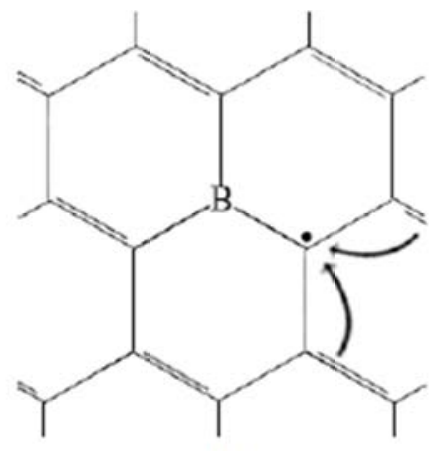

(b)

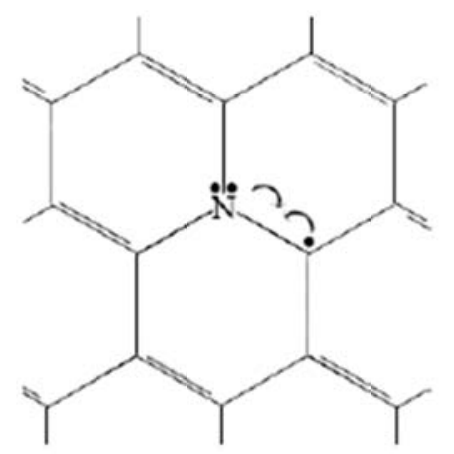

(c) 
Figure 38
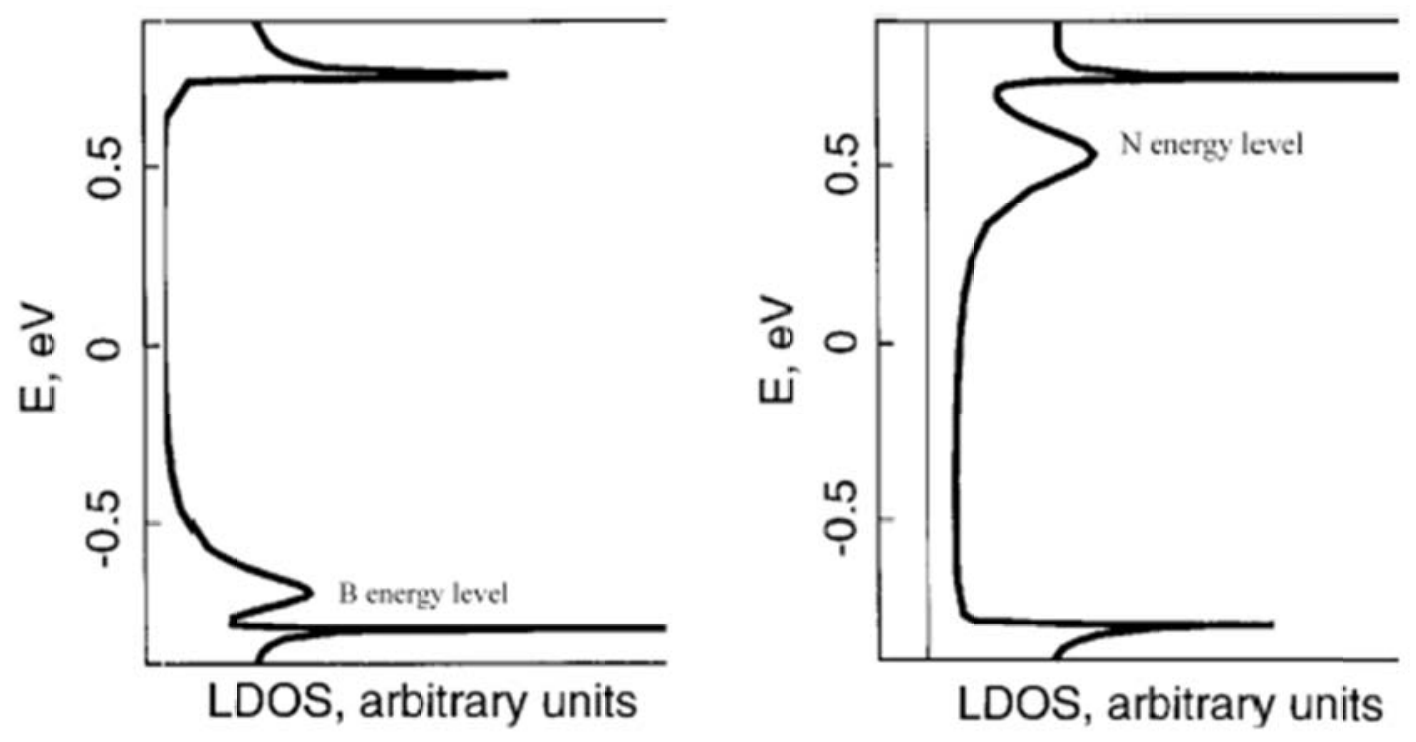

(a)

(b) 
Figure 39

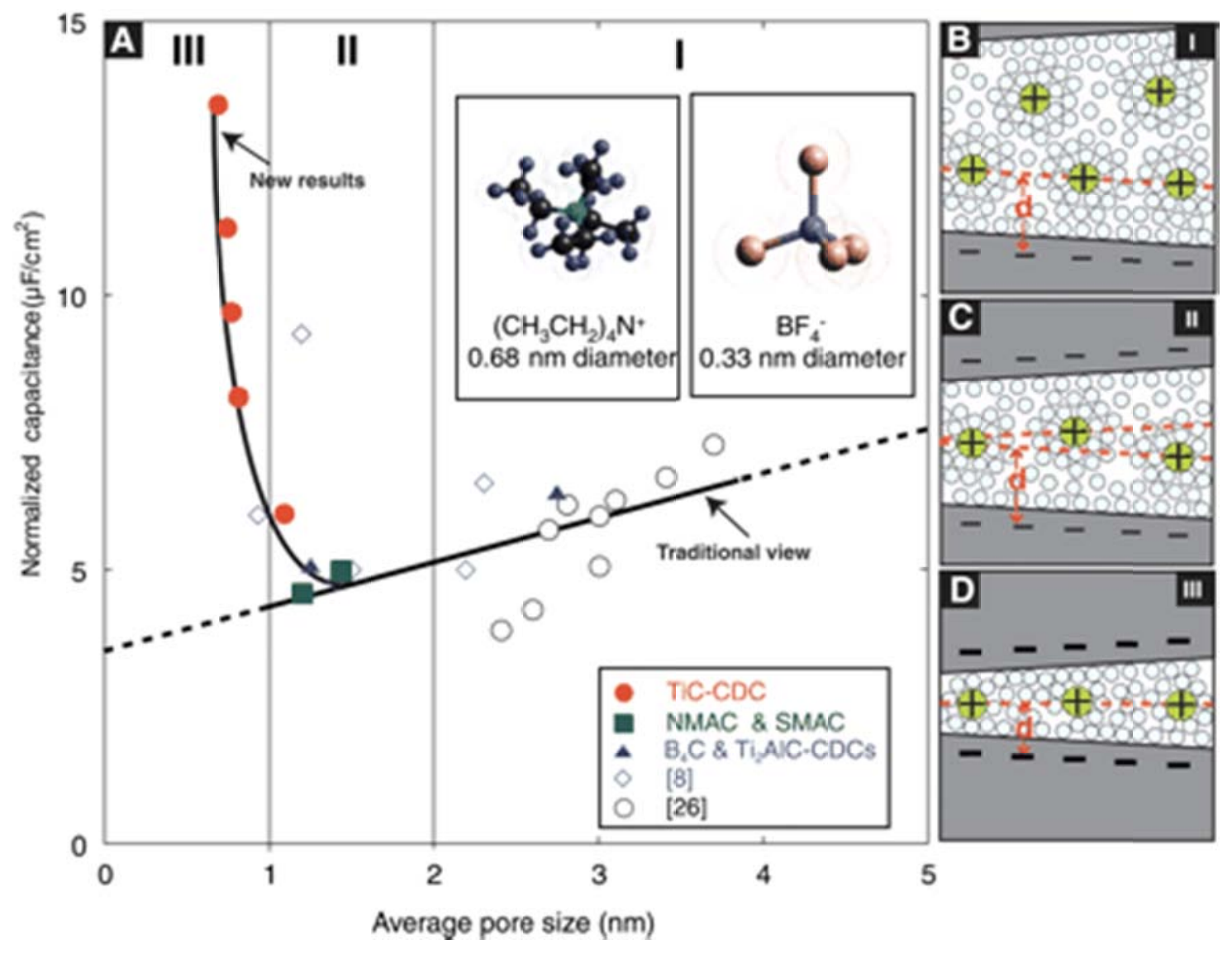


Figure 40

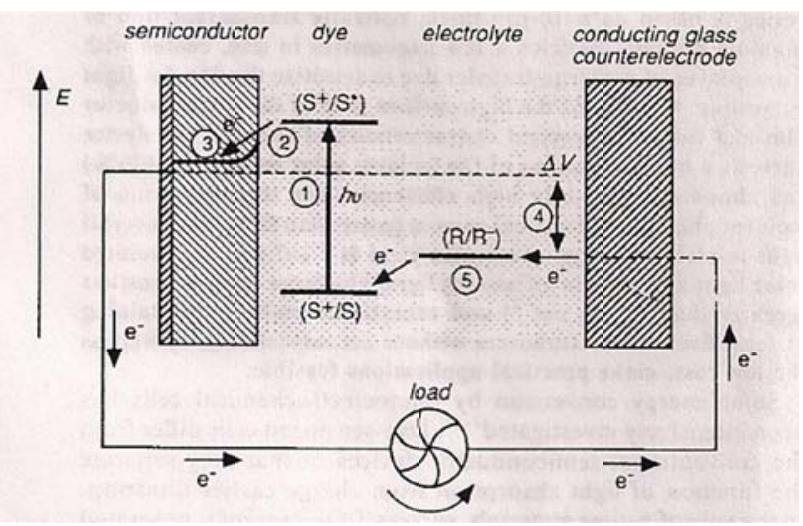


Figure 41
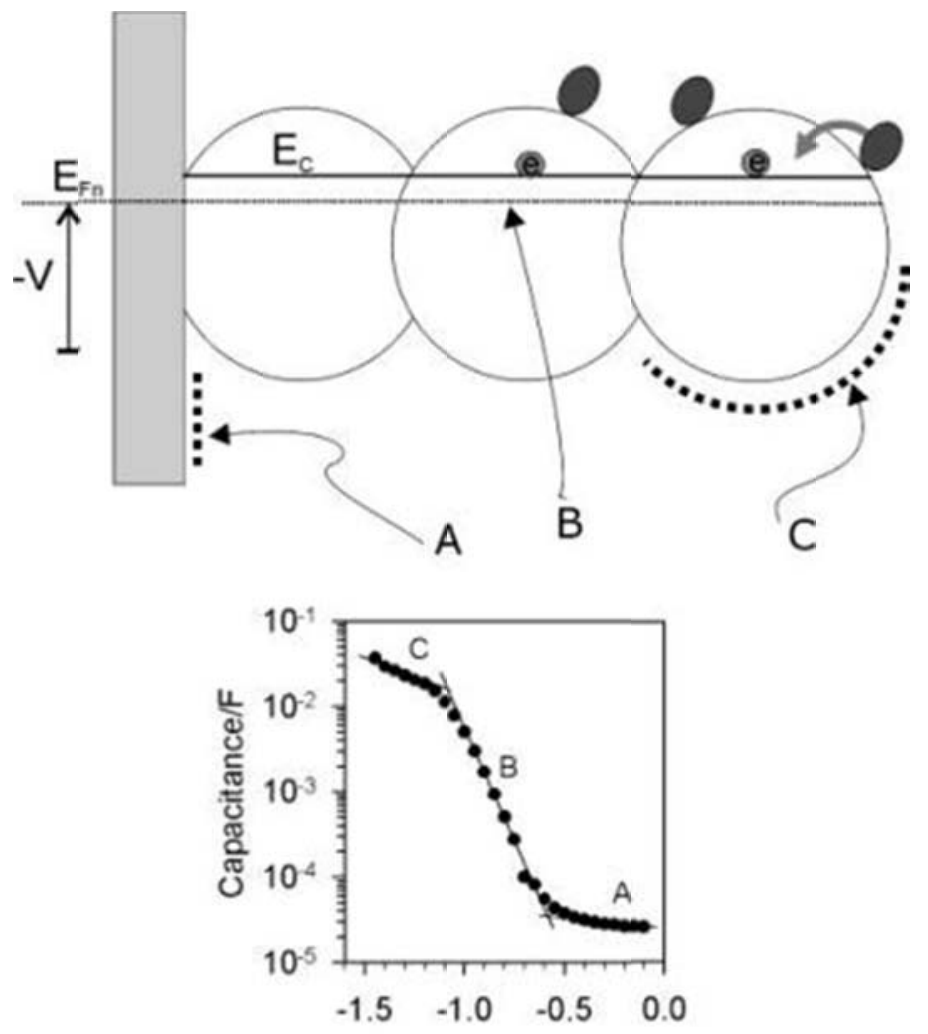

Potential (V vs Ag/AgCl) 
Figure 42

(a)
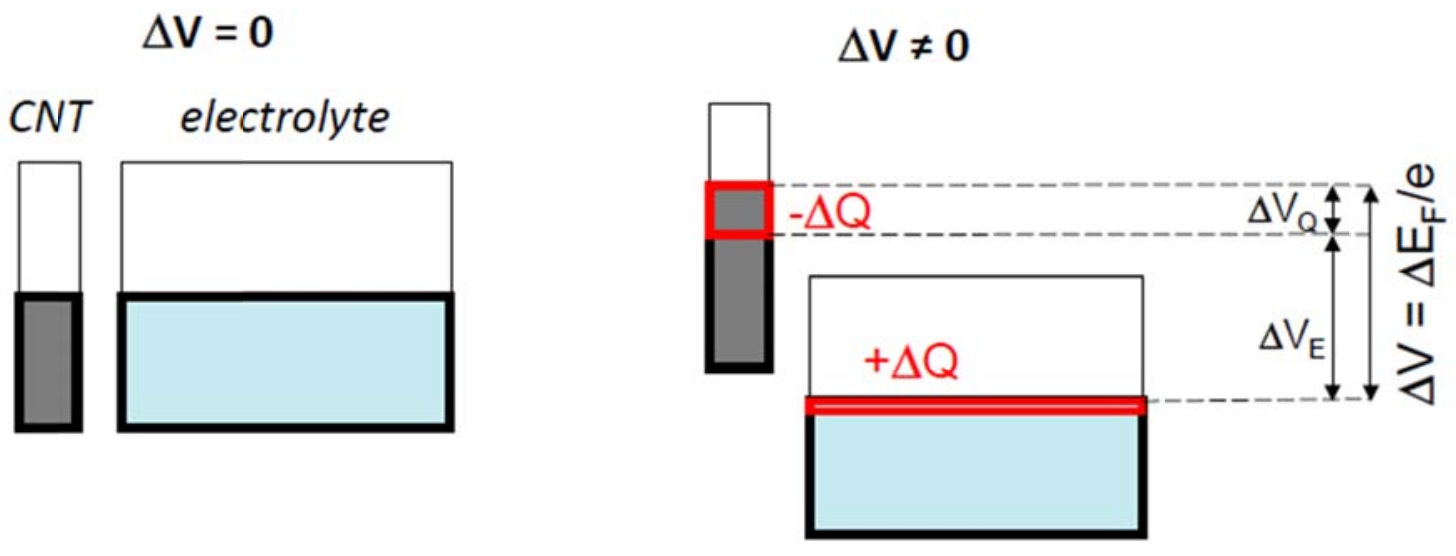

(b)

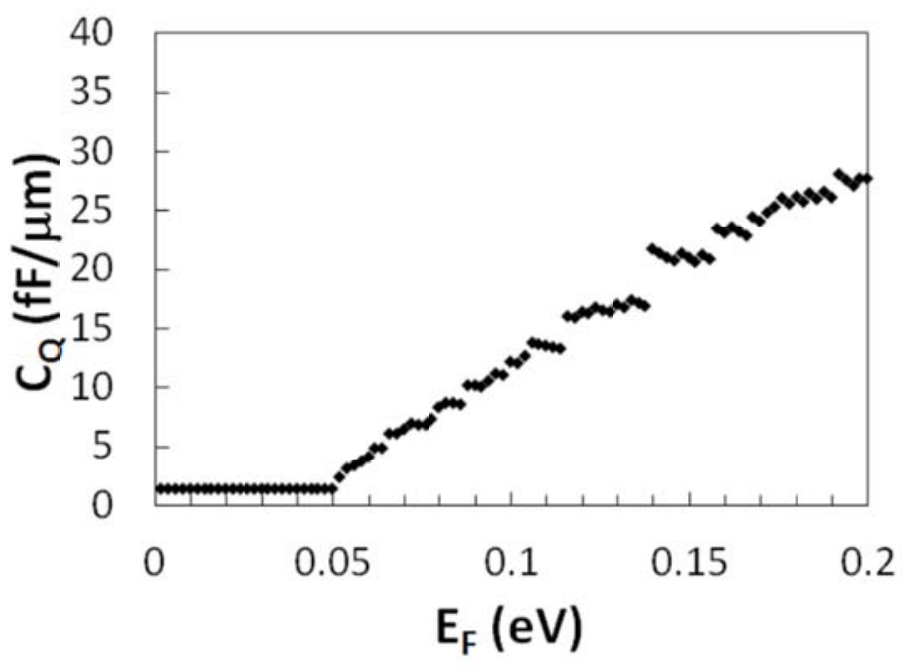




\section{Figure 43}

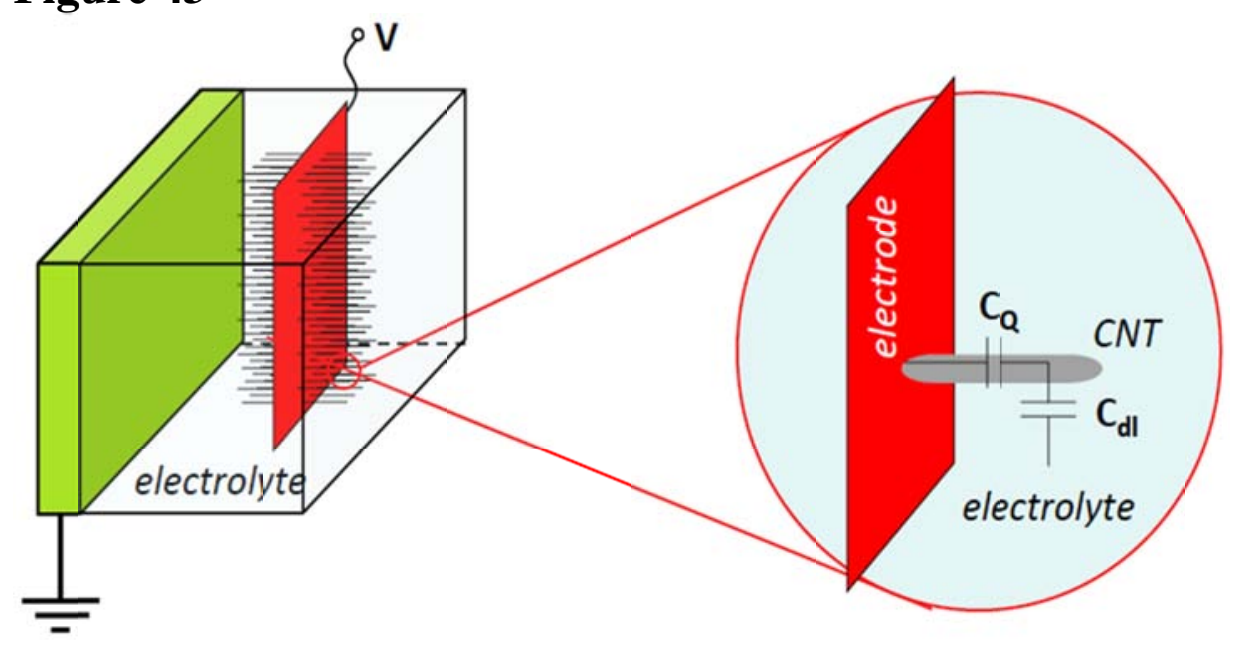


Figure 44

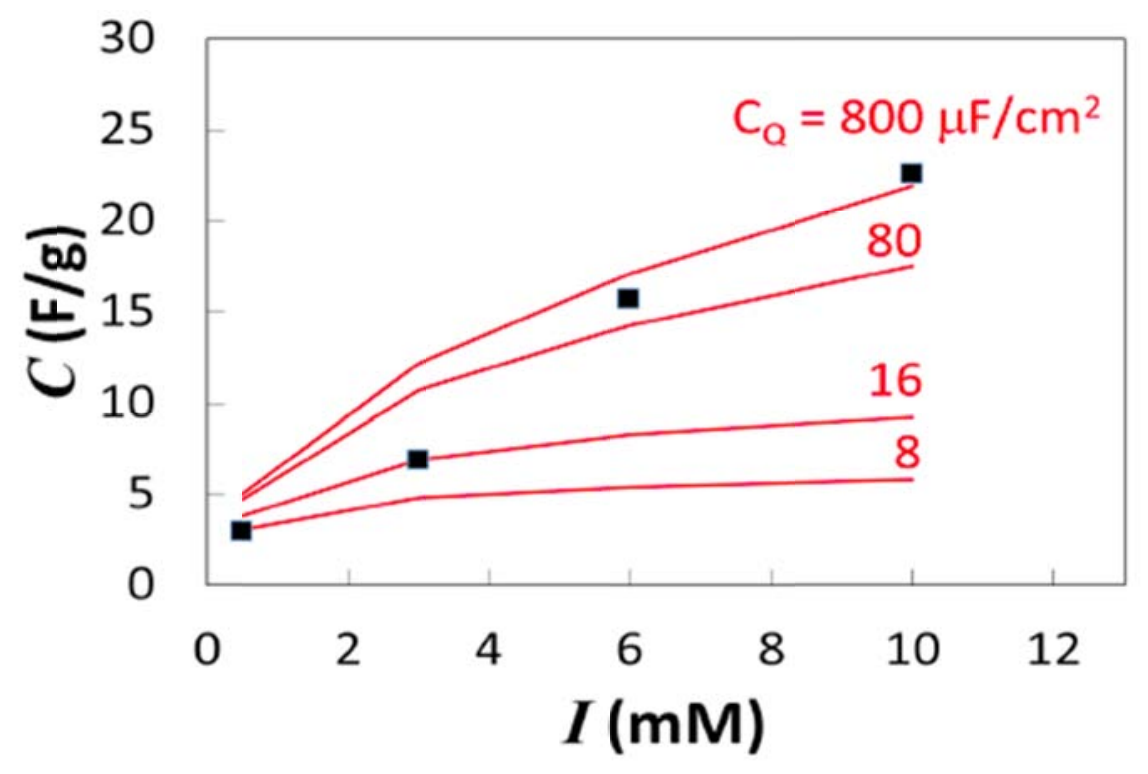


Figure 45

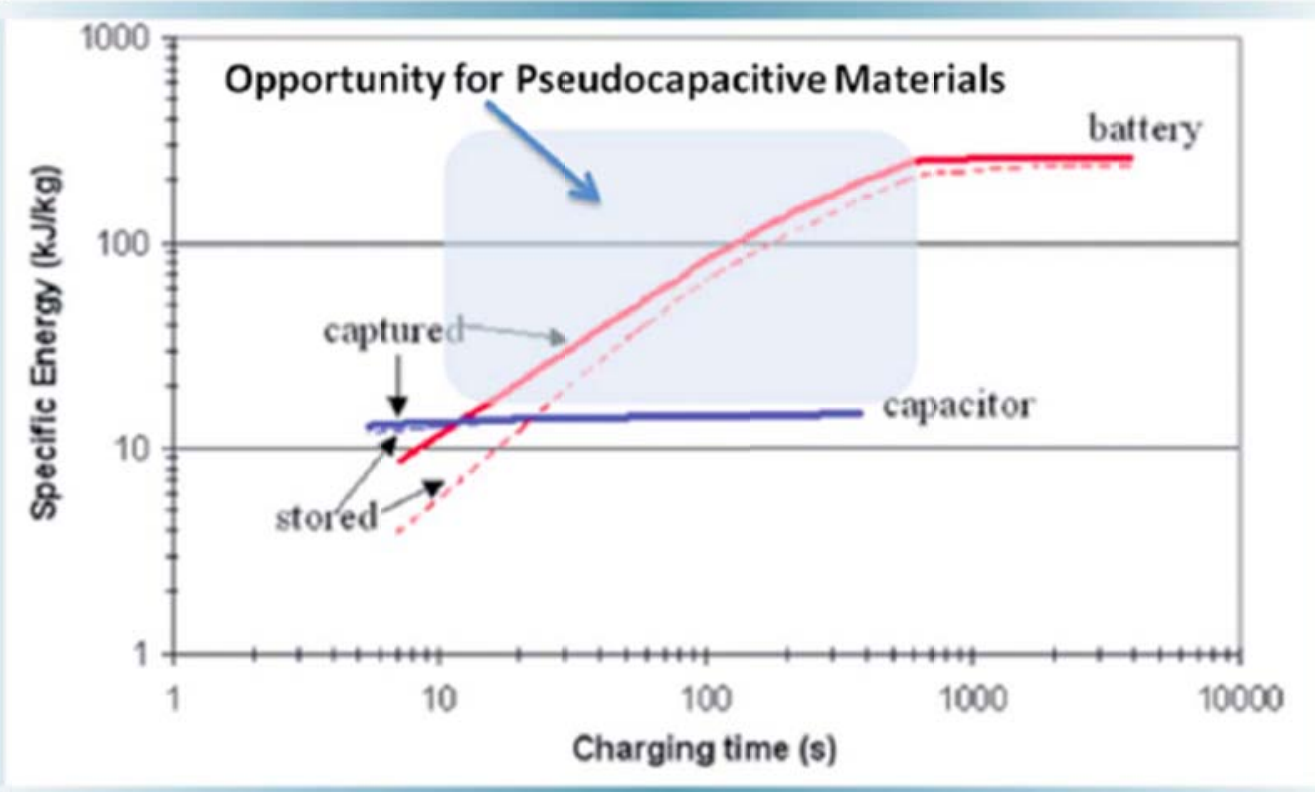


Figure 46

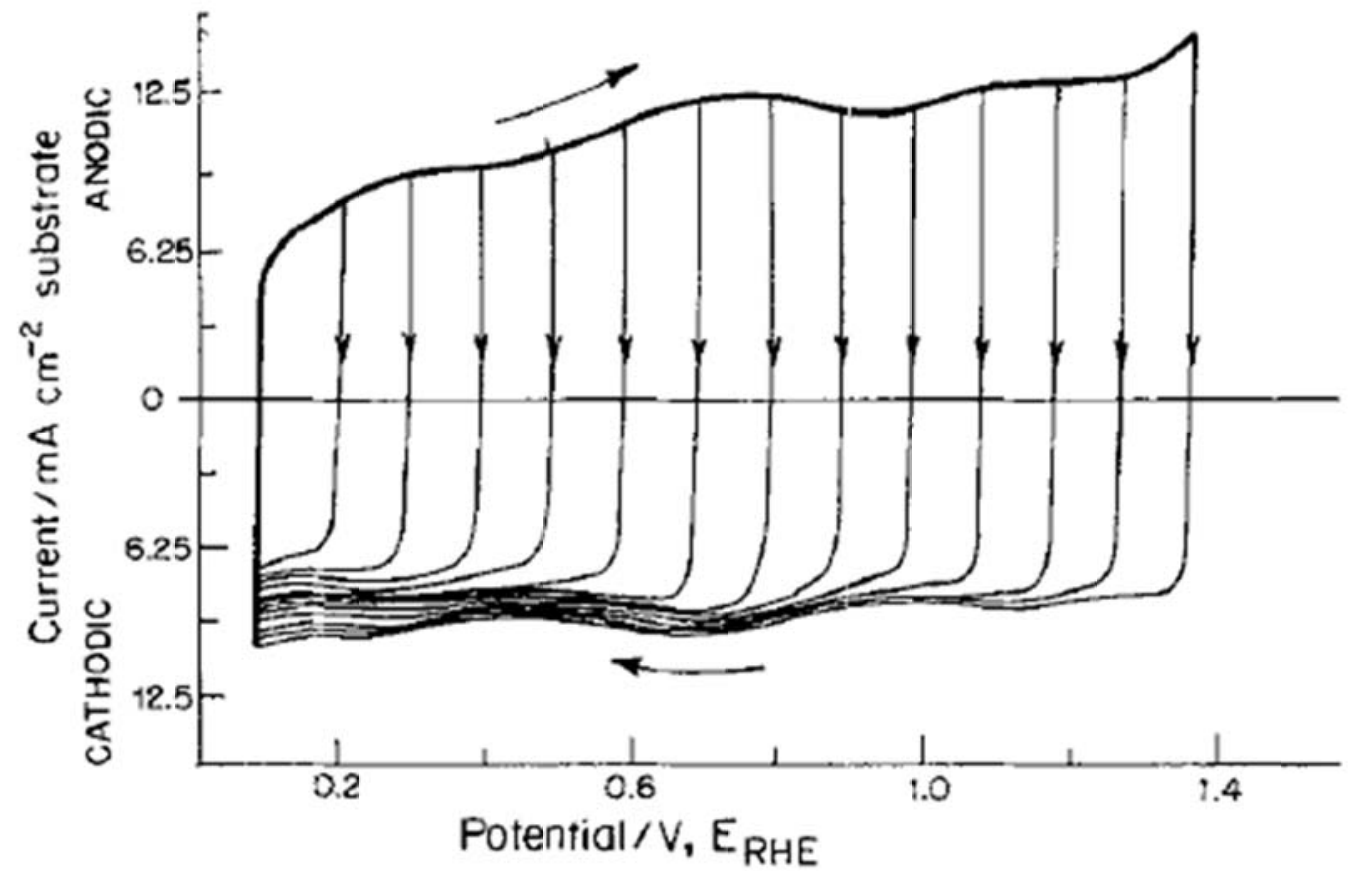


Figure 47
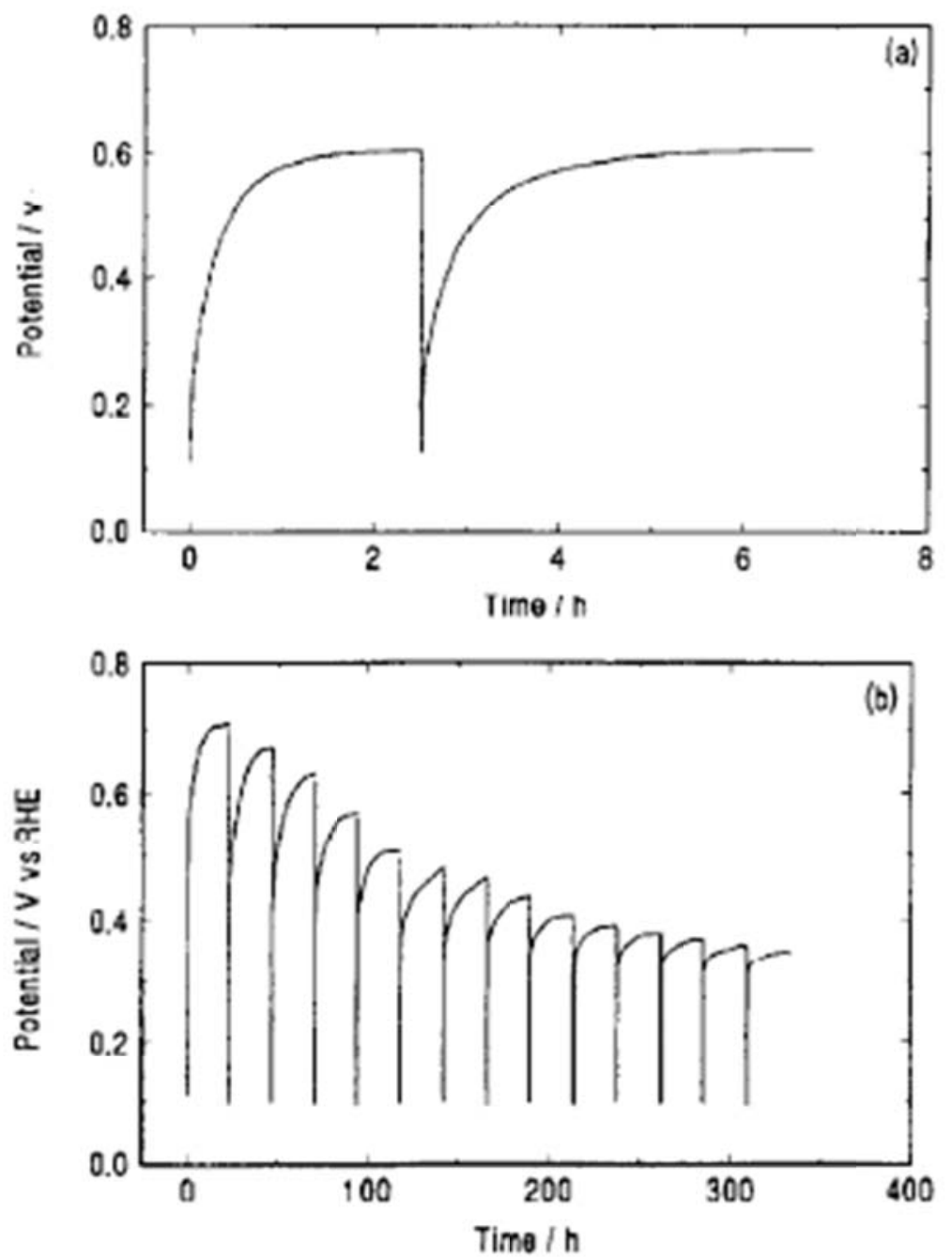
Figure 48

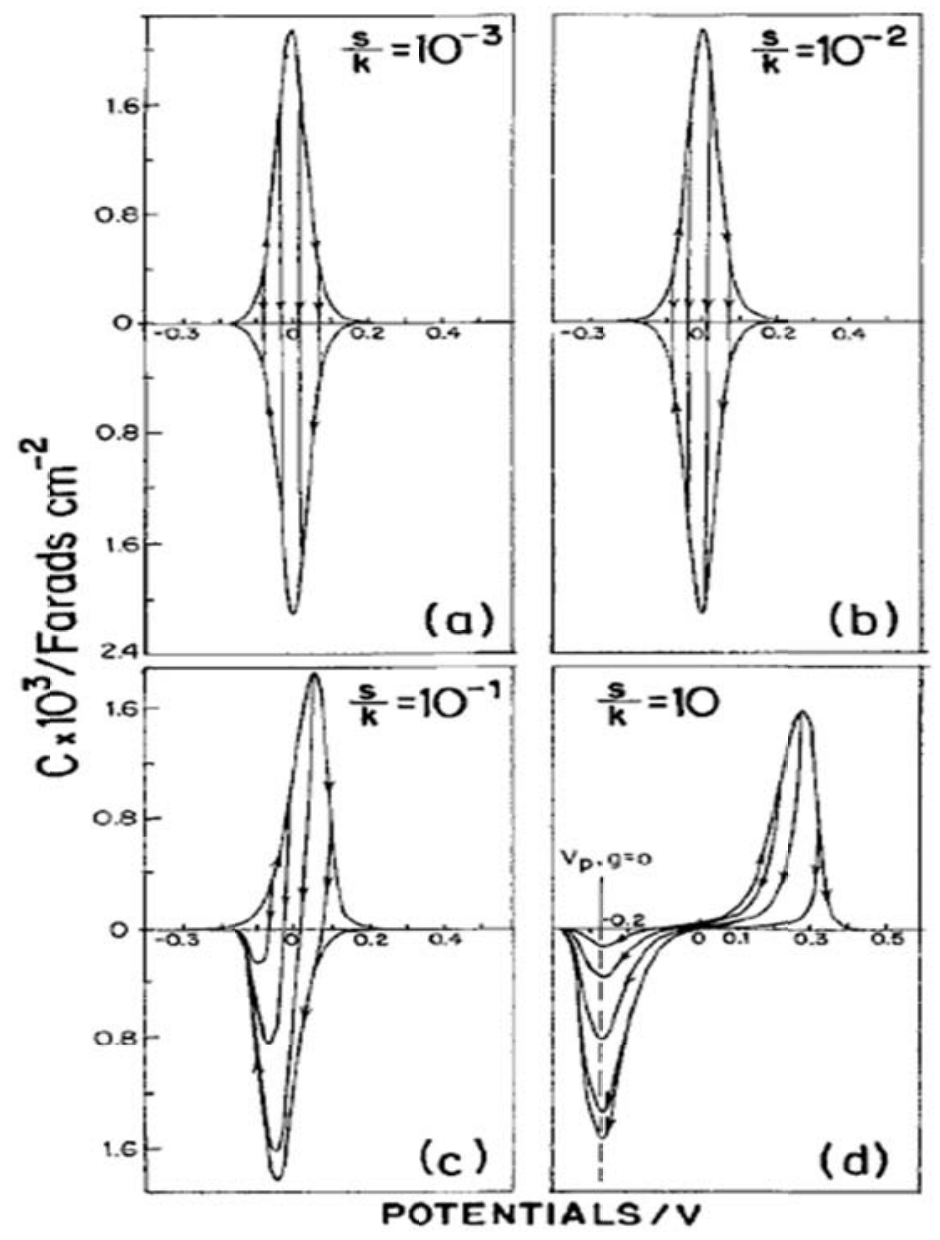


Figure 49

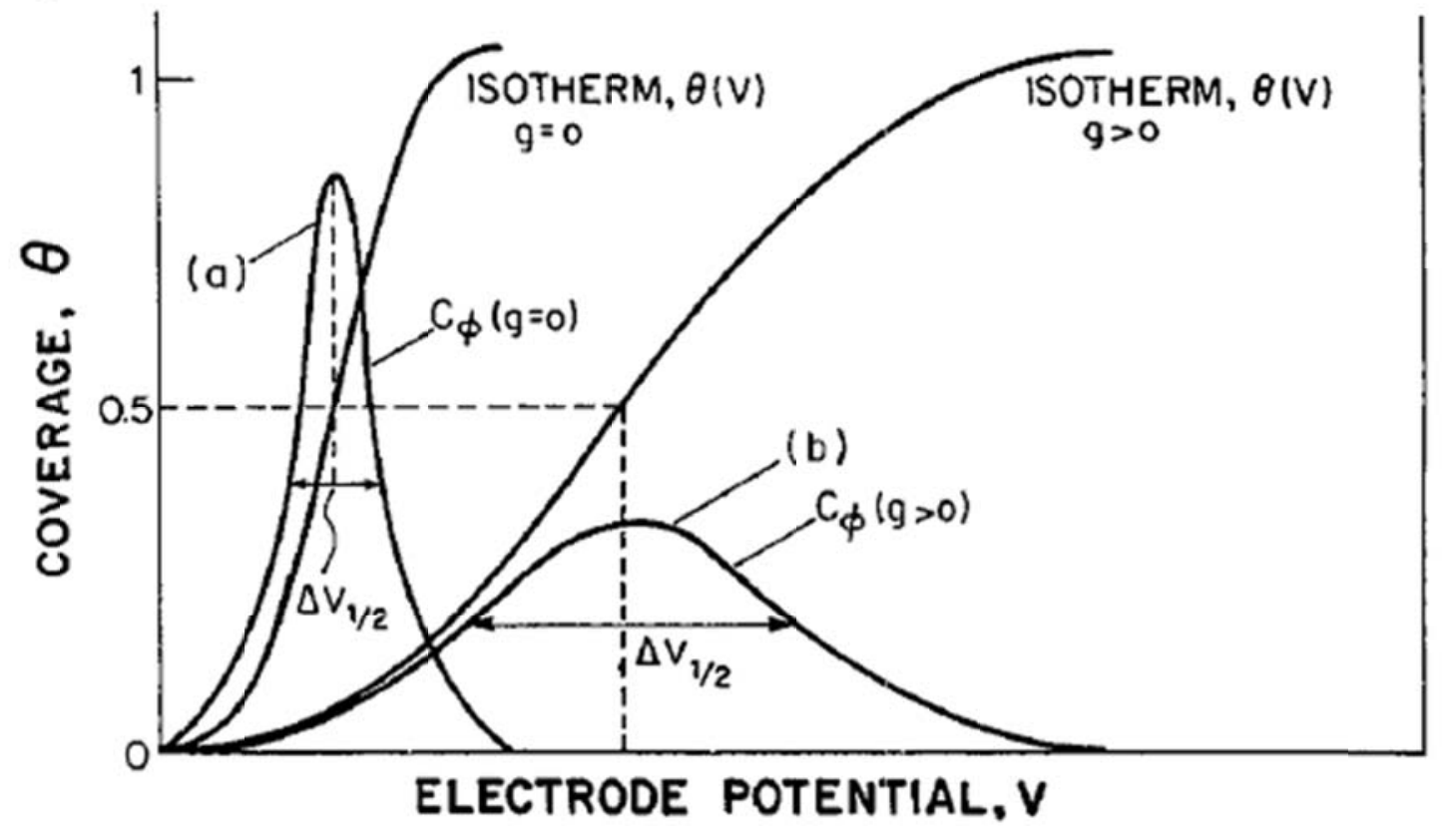


Figure 50
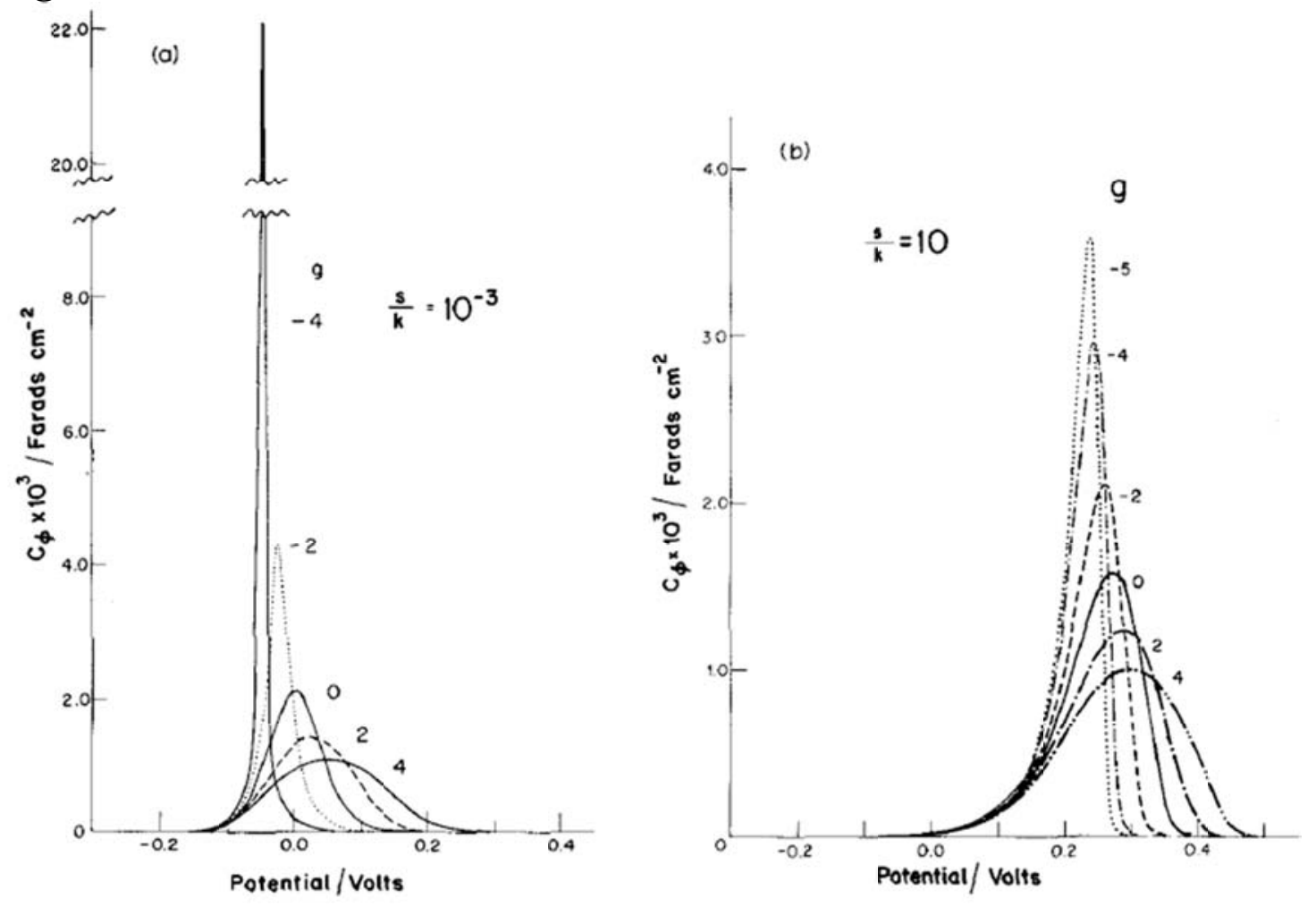

Page 307 of 317 
Figure 51

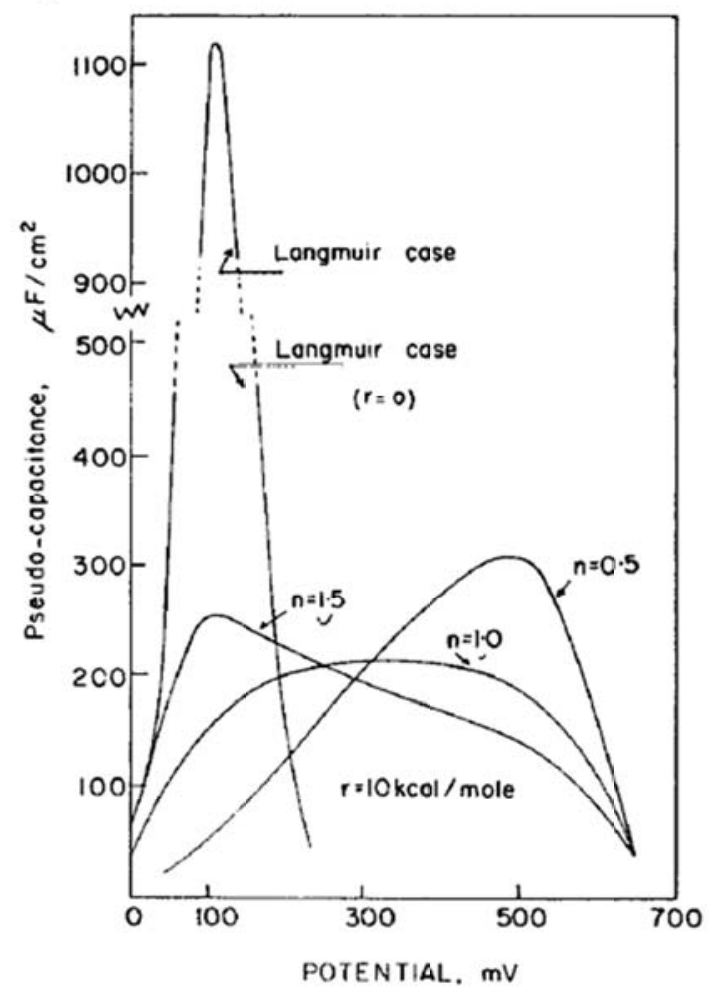

Page 308 of 317 
Figure 52

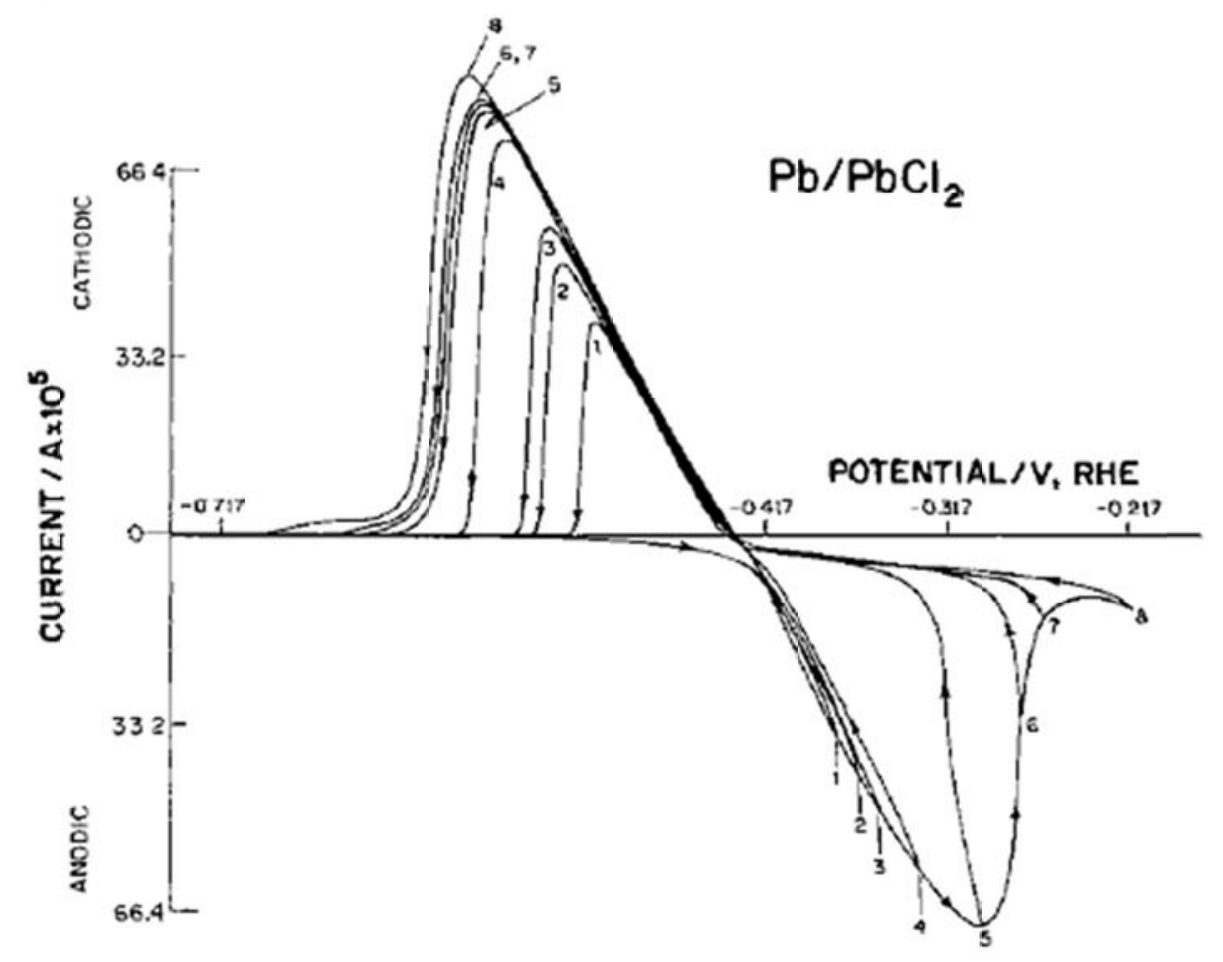




\section{Figure 53}

(a)
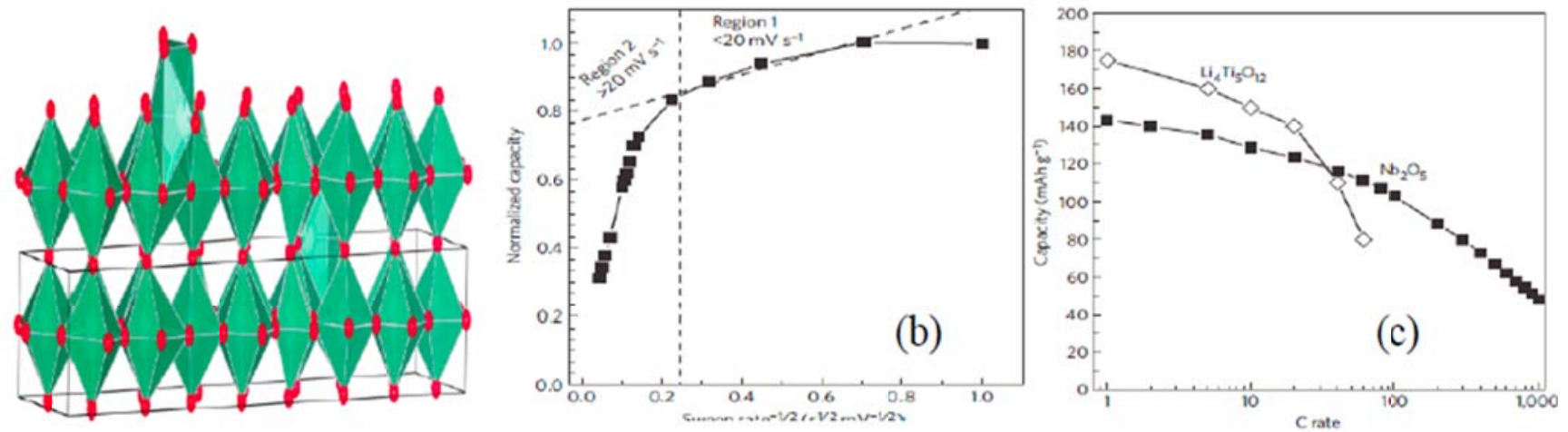
Figure 54
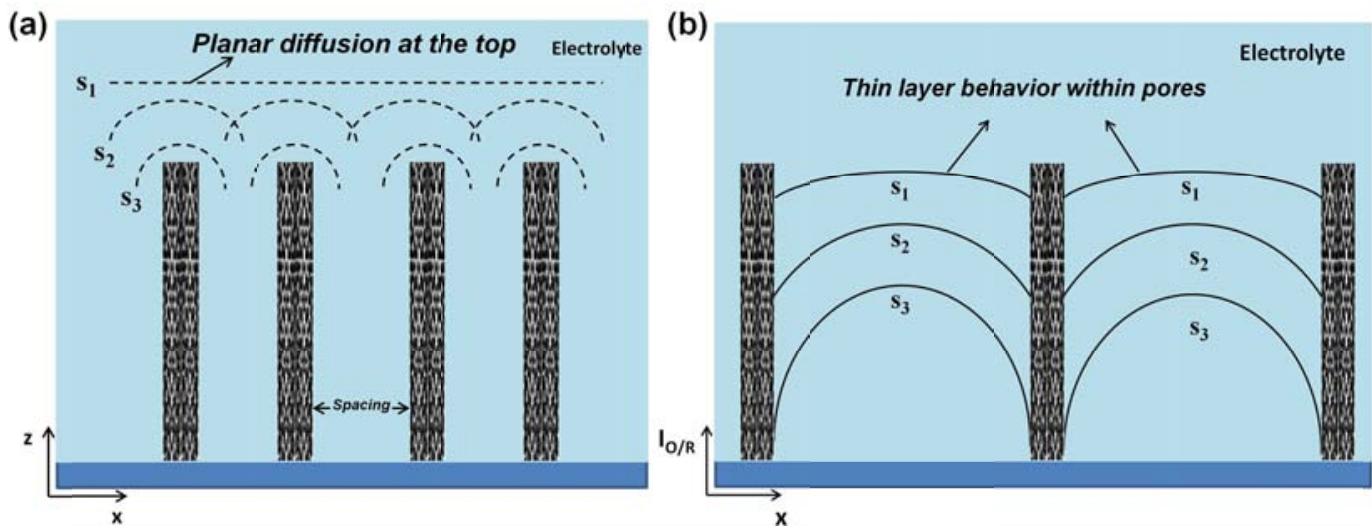
Figure 55
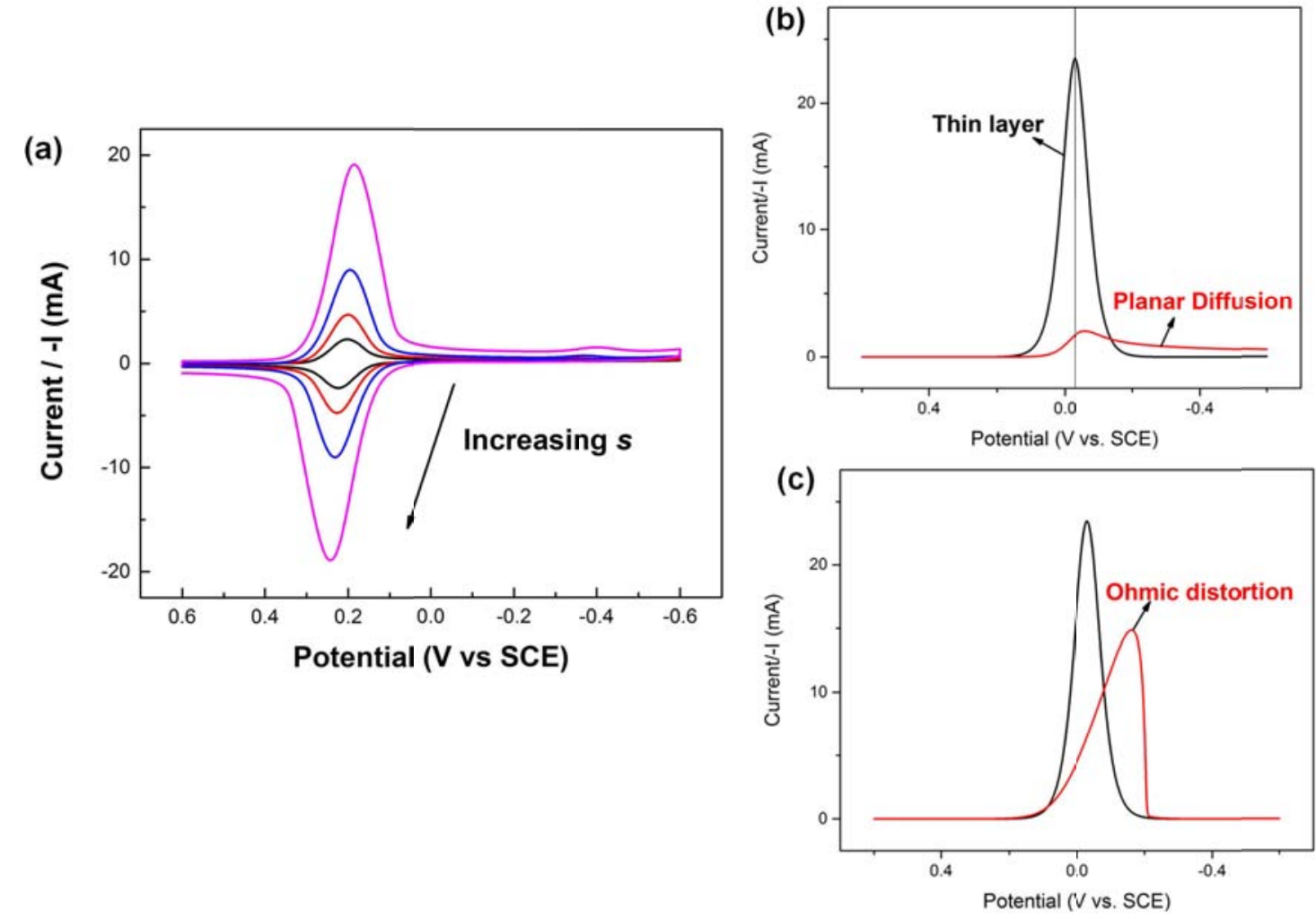
Figure 56
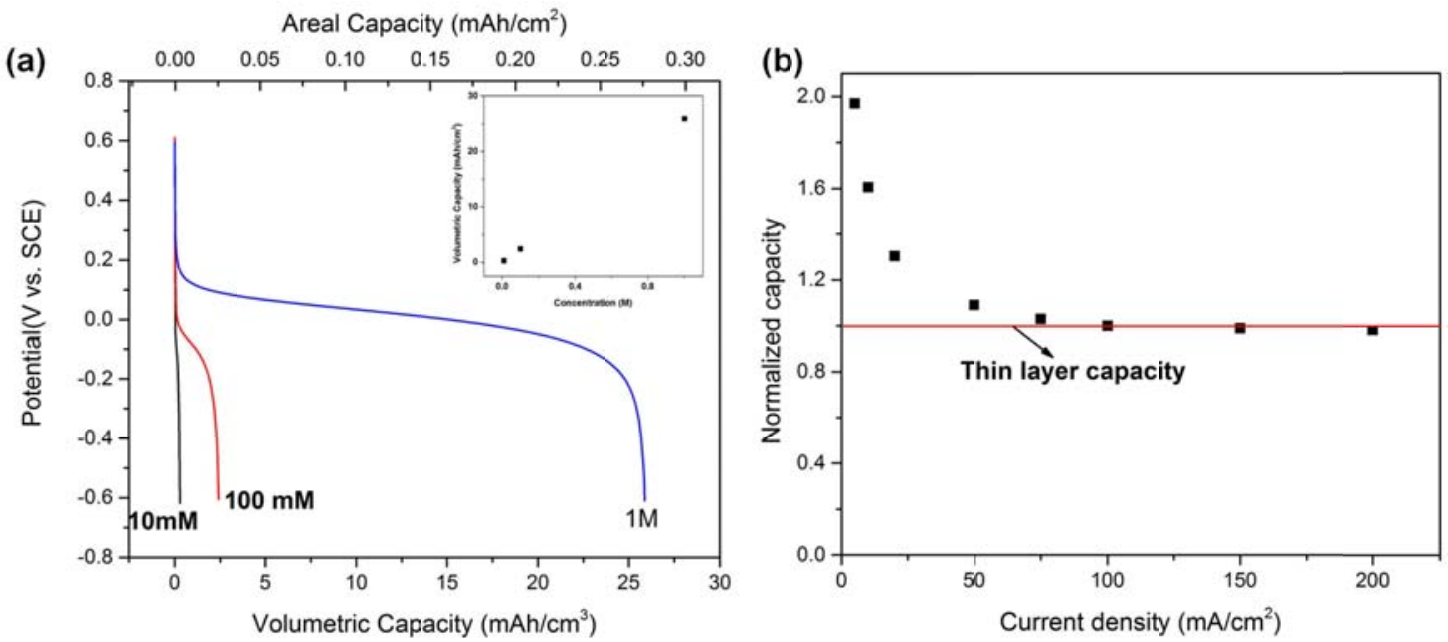
Figure 57
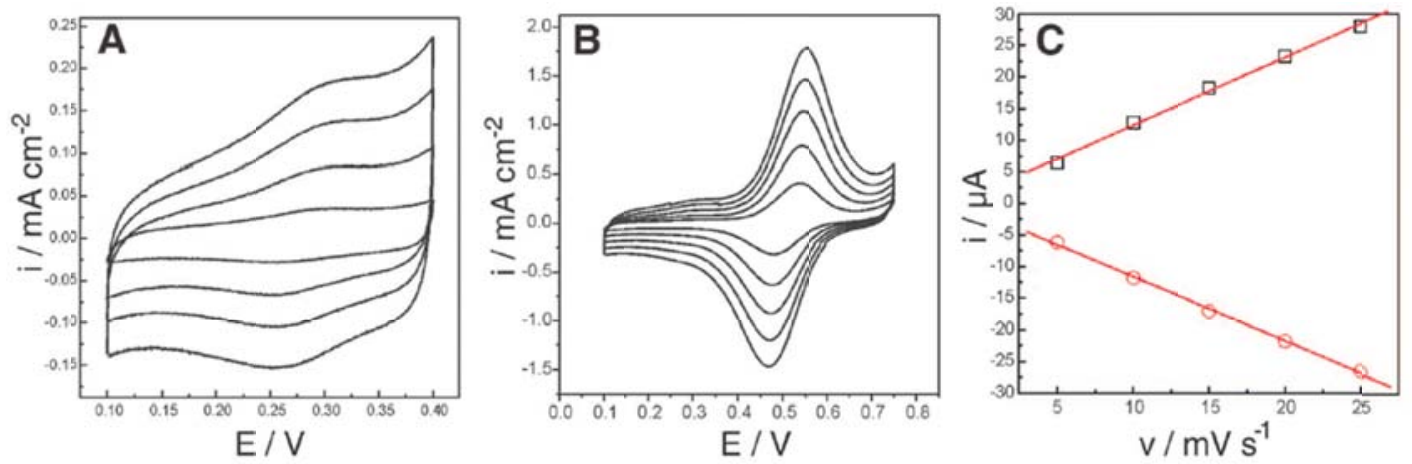
Figure 58

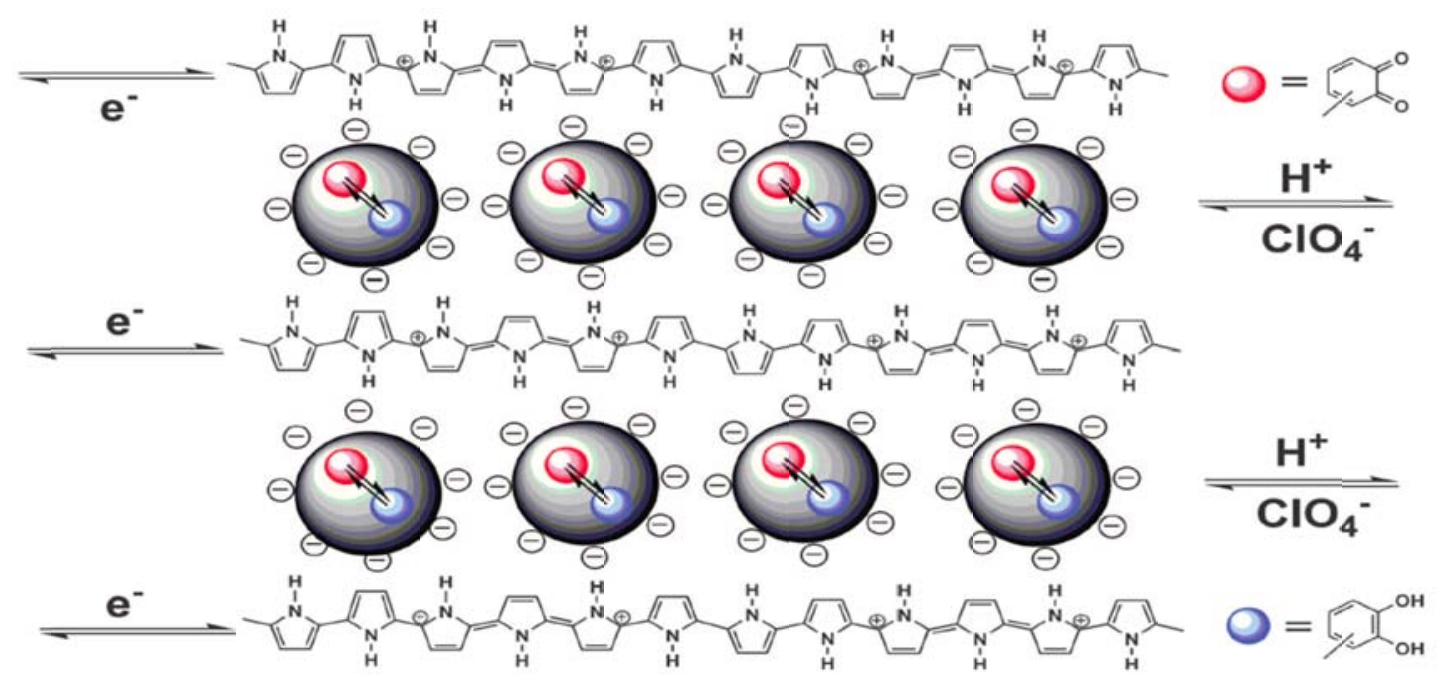


Figure 59

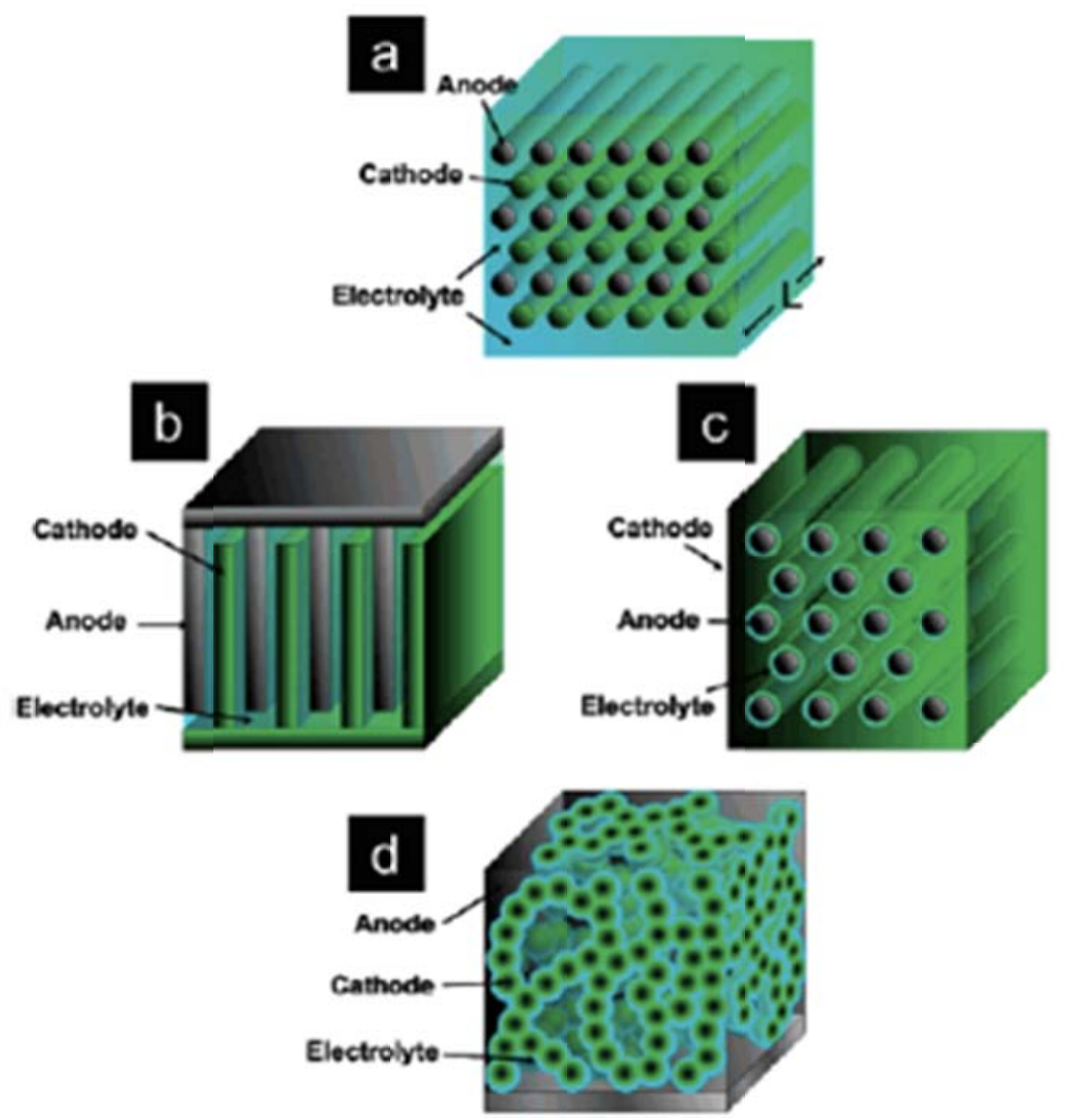


Figure 60

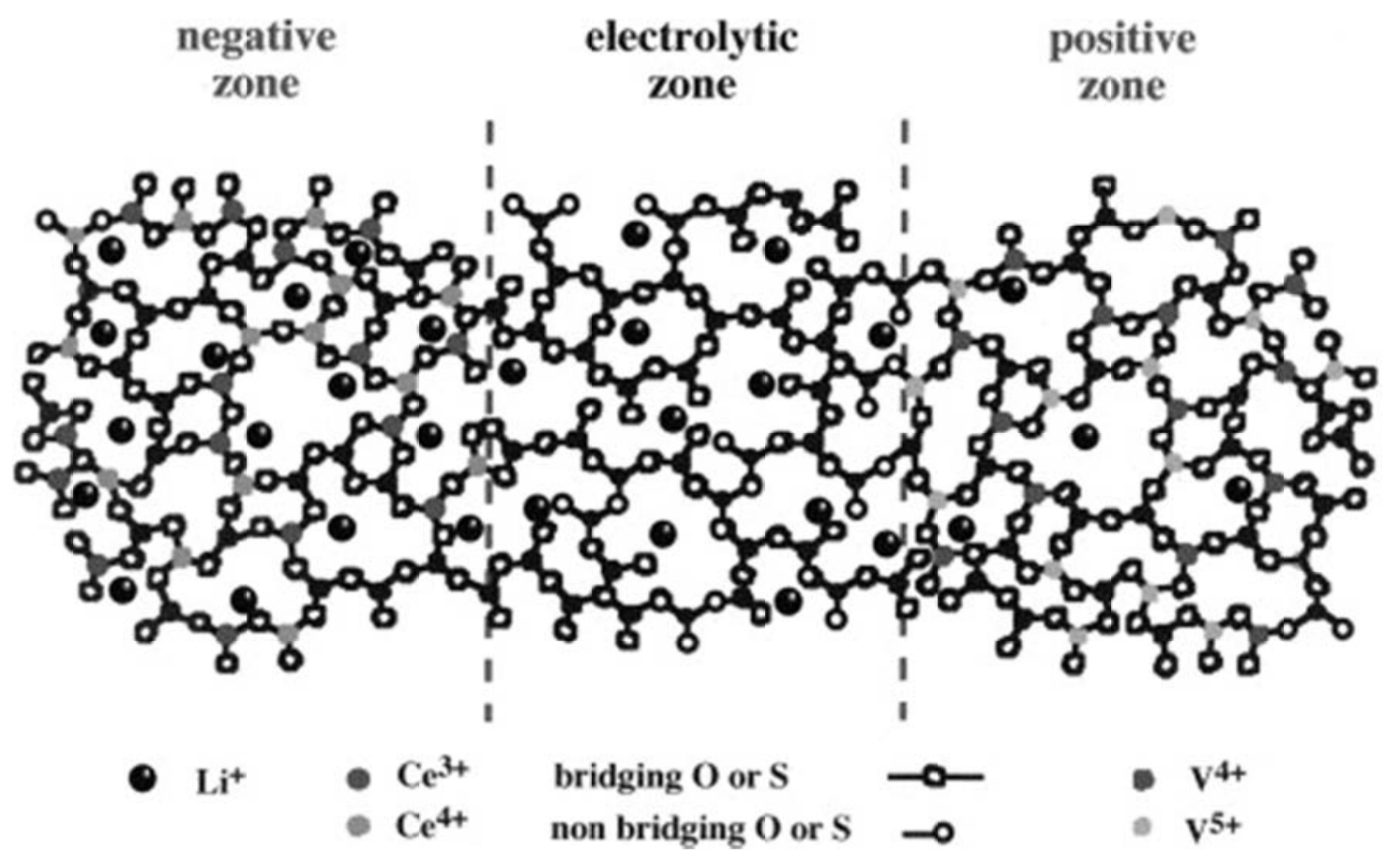

